\title{
THE MORPHOLOGICAL RANGE IN MALLOTUS (EUPHORBIACEAE) AND A TAXONOMIC REVISION OF ITS SECTION ROTTLEROPSIS (INCLUDING AXENFELDIA) IN MALESIA, THAILAND AND AFRICA
}

\author{
S.E.C. SIERRA, M. APARICIO, M.J.H. GEBRAAD, \\ K.K.M. KULJU \& P.C. VAN WELZEN \\ Nationaal Herbarium Nederland, Universiteit Leiden branch, \\ P.O. Box 9514, 2300 RA Leiden, The Netherlands; \\ e-mail: sierra@nhn.leidenuniv.nl,welzen@nhn.leidenuniv.nl
}

\begin{abstract}
SUMMARY
The range of diagnostic morphological features in the whole genus Mallotus is reviewed as background to the taxonomic treatment of section Rottleropsis s.l. Special emphasis is given to the indumentum, habit, stipules, leaves, extrafloral nectaries, domatia, inflorescences, flowers, pollen and fruits. Notes on the geographical distribution, ecology, pollinators, fruit and seed dispersal, and uses of the genus are also presented. The section Axenfeldia is reduced to sect. Rottleropsis on account of the absence of suitable morphological characters to distinguish them. A taxonomic revision of 38 species of sect. Rottleropsis s.l. from Malesia, Thailand and Africa is presented, together with regional and synoptical identification keys, distribution maps and illustrations. Four new species are described: Mallotus connatus, M. longinervis, M. minimifructus and M. mirus. Several non-Malesian species not included in this revision are briefly discussed. This paper concludes a series of taxonomic revisions of the genus Mallotus in Malesia and Thailand.
\end{abstract}

Key words: Euphorbiaceae, Axenfeldia, Mallotus, Rottleropsis, Malesia, Thailand, Africa.

\section{INTRODUCTION}

The classification of the species in the genus Mallotus Lour. (Euphorbiaceae) has been modified by different authors since Müller Argoviensis (1865) revised the genus and proposed three sections. In the latest classification by Airy Shaw (1968) eight sections were recognized: Axenfeldia (Baill.) Pax \& K. Hoffm., Hancea Pax \& K. Hoffm., Mallotus, Oliganthae Airy Shaw, Polyadenii Pax \& K. Hoffm., Rottlera (Willd.) Rchb.f. \& Zoll., Rottleropsis Müll.Arg. and Stylanthus (Rchb.f. \& Zoll.) Pax \& K. Hoffm. Several of these sections were recently revised, viz. Polyadenii (Bollendorff et al., 2000), Hancea (Slik \& Van Welzen, 2001a)1, Stylanthus (Slik \& Van Welzen, 2001a), Philippinenses (= Mallotus sect. Rottlera) (Sierra et al., 2005; Sierra \& Van Welzen, 2006), Mallotus (Sierra \& Van Welzen, 2005), and Oliganthae (= Cordemoya sect. Oliganthae) ${ }^{1}$ (Sierra et al., 2006). Only Axenfeldia and Rottleropsis still require revision.

1) Based on results of phylogenetic research by Kulju et al. (in press), Mallotus sect. Hancea and sect. Oliganthae have been transferred from Mallotus s.l. to Cordemoya. Mallotus sect. Hancea and sect. Oliganthae are nowadays Cordemoya sect. Diplochlamys and sect. Oliganthae, respectively (Slik \& Van Welzen, 2001a; Sierra et al., 2006). 
The composition of the sections has undergone many changes. Pax \& Hoffmann (1914) proposed Mallotus sections Echinocroton and Plagianthera based on the absence or presence of spiny fruits, respectively. The name Echinocroton was published on the genus level by Mueller (1858). However, Pax \& Hoffmann gave it incorrectly precedence over the sectional name Rottleropsis, which they treated as a synonym of sect. Echinocroton. Airy Shaw (1968) did not recognize the sections Echinocroton and Plagianthera as different groups, and decided to merge them using the sectional name Rottleropsis because it was the earliest name available on the sectional rank level.

Based on the genus Axenfeldia Baill. (1858), Mallotus sect. Axenfeldia was proposed by Pax \& Hoffmann (1914). Pax \& Hoffmann included atypical species of several existing sections (Rottlera sect. Stylanthus Rchb.f. \& Zoll., Mallotus sect. Melanolepis Müll.Arg. and Mallotus sect. Eumallotus Müll.Arg.), but afterwards other authors placed some of these species in other sections or genera.

The circumscriptions of sections Axenfeldia and Rottleropsis, as proposed by Airy Shaw $(1968,1972)$, were based on differences in leaf venation: pinnate in Axenfeldia and tripli- or palminerved in Rottleropsis. However, in his initial publication, Airy Shaw (1968) already indicated that by using the leaf venation character to distinguish between the two sections, these were not 'sharply demarcated'. This problem also surfaced in the present revisional work. When studying the venation pattern of $M$. claoxyloides, $M$. darbyshirei, and M. macularis we noticed that the basal nerves that branch from the base of the midrib in most cases are faint which is not the case in other Mallotus species of these sections. We opted to take these faint nerves into account describing the pattern as 'inconspicuously triplinerved'. However, if these faint nerves would not have been taken into account the description of the pattern for these three species would have been pinnate. Furthermore, revisional work on sections Polyadenii (Bollendorff et al., 2000) and Stylanthus (Slik \& Van Welzen, 2001a), has shown that the venation character is very variable. Apart from the 'homoplaceous' leaf venation character, the revision of sections Axenfeldia and Rottleropsis revealed no clear suitable characters to distinguish between them. Additionally, phylogenetic analyses based on morphological (Slik \& Van Welzen, 2001b) and molecular data (Kulju et al., in press), suggested that both sections are polyphyletic (with species from both sections intermixed), and that the venation characters should be considered homoplastic. However, both phylogenies suffer from insufficient taxon sampling for the Mallotus s.s. clade (including both sections dealt with here), and from low support for the resulting clades. Therefore, these phylogenetic results could not be used to propose a new classification of sections Axenfeldia and Rottleropsis. These results do show that most likely both options of merging the sections or keeping them as two separate sections, would still have implied unnatural groups. Taking all evidence into account we have studied the species of both sections simultaneously during the revisional work. Additionally, because there are no suitable characters that can be used to distinguish sections Axenfeldia and Rottleropsis they are synonymized. Based on priority rules Rottleropsis is the accepted name and Axenfeldia is a synonym. Noted is that the decision of merging them into one large section most likely does not solve the problem entirely and that sect. Rottleropsis s.l. needs further subdivision, once a phylogeny can provide supported clades. Rottleropsis s.l. shares the following combination of characters: the presence of unequal opposite leaves, usually with glandular hairs on the lower surface and fruits that are dehiscent to tardily dehiscent capsules or indehiscent and drupaceous. 
Two other publications treat additional taxa (not dealt with here) belonging to sect. Rottleropsis s.1. The first one, by Van Welzen \& Sierra (2006), describes four species known as the Wrayi complex. The second one, by Kulju et al. (2007), revises four species formerly placed in other genera of the subtribe Rottlerinae. Based on the molecular studies by Kulju et al. (in press) these genera were found to belong to Mallotus and their morphology suggests that some of them are part of sect. Rottleropsis s.l. (Kulju et al., 2007): M. cumingii Müll.Arg. (was Neotrewia cumingii (Müll.Arg.) Pax \& K. Hoffm.), M. nudiflorus (L.) Kulju \& Welzen (was Trewia nudiflora L.) and M. polycarpus (Benth.) Kulju \& Welzen (was Trewia polycarpa Benth.).

Furthermore, the morphological phylogeny by Slik \& Van Welzen (2001b) suggested that four species (M. brachythyrsus Merr., M. havilandii Airy Shaw, M. insularum (Airy Shaw) Slik, M. miquelianus (Scheff.) Boerl.), previously assigned to Mallotus sect. Hancea, should be included in sect. Axenfeldia and Rottleropsis, also based on the presence of opposite leaves, unequal in size. In the phylogeny by Kulju et al. (in press) they are part of the Mallotus s.s. clade, but since their relationship with other sections remains unclear, we do not consider them to be part of section Rottleropsis s.l. The revision of the former Hancea species has been treated in Slik \& Van Welzen (2001a) and Van Welzen et al. (2006; including a fifth species, M. concinnus Airy Shaw).

The purpose of this paper is to review the range of diagnostic morphological features in the whole genus Mallotus and to provide a revision of the remaining species of section Rottleropsis s.1., including keys, descriptions for 38 taxa occurring in Malesia, Thailand and Africa, distribution maps and illustrations. Until a complete phylogenetic reconstruction based on molecular and morphological evidence is available, we provisionally accept, to some extent, the traditional sectional composition as circumscribed by Airy Shaw.

\section{NOTES ON MORPHOLOGICAL CHARACTERS, DISTRIBUTION AND ECOLOGY OF MALLOTUS}

Descriptions and measurements are based on dry herbarium material. The descriptive terms for the shapes of stipules, leaves and sepals include the length/width ratios: A ratio of c. 1 is called broadly ovate, orbicular, broadly obovate or deltoid; a ratio of $1-3$ is called ovate, elliptic, obovate or triangular; a ratio of $3-5$ is called narrowly ovate, narrowly elliptic, narrowly obovate or narrowly triangular, and a radio of $>5$ is called linear-ovate, linear-elliptic, linear-obovate or linear-triangular. When a feature is not mentioned in the descriptions, then it is absent. In the descriptions and keys, the non-glandular indument is always described first, and the glandular indument second. Label information was used to infer distributions, ecology, altitudes and uses.

\section{Habit}

Most of the Mallotus species are shrubs or trees, rarely climbers (M. repandus). Mallotus leucodermis Hook.f. and M. chromocarpus Airy Shaw reach sizes up to 54 and $43 \mathrm{~m}$ high, respectively, whereas species like M. coudercii (Gagnep.) Airy Shaw do not grow higher than c. $0.60 \mathrm{~m}$. Most species are evergreen, some are deciduous (e.g., M. khasianus Hook.f., M. nudiflorus, M. polycarpus). 

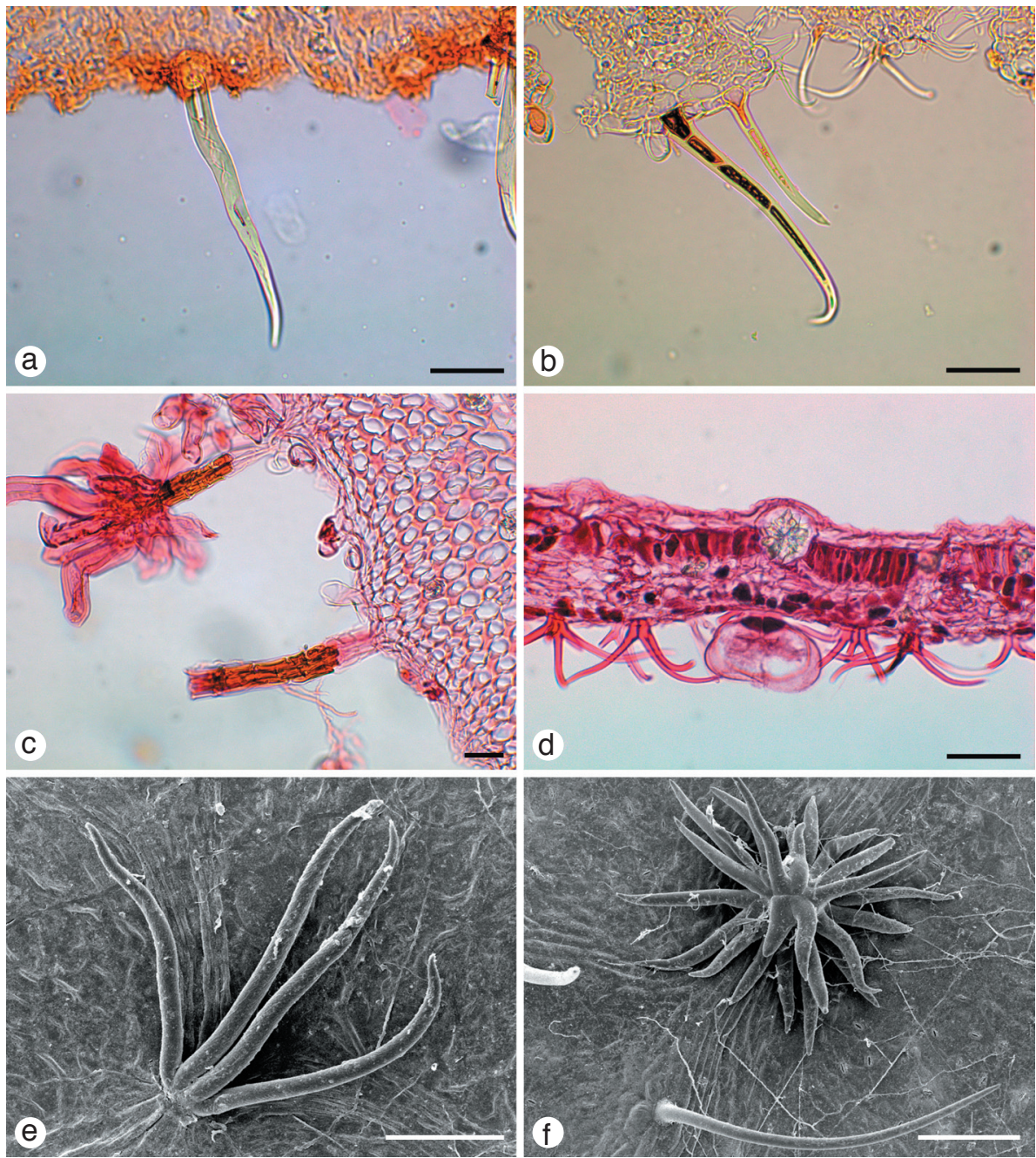

Fig. 1. Indumentum of Mallotus. a. Simple unicellular hair of M. cauliflorus Merr.; b. simple multicellular hairs of M.philippensis (Lam.) Müll.Arg.; c. stellate hairs with long stalk in M.macrostachyus (Miq.) Müll.Arg.; d. tufted hairs of M.rhamnifolius Müll.Arg.; e. tufted hair of M.polycarpus (Benth.) Kulju \& Welzen; f. 3-dimensionally radiating stellately-tufted hair of M. tiliifolius (Blume) Müll. Arg. (a: BS (Ramos \& Edaño) 33460; b: SAN (S. Lantoh) 73452; c: KEP FRI (A.K. Cheng) 27524; d: Jayasuriya 1283; e: Stocks et al. s.n. (without barcode); f: Unknown s.n. (barcode L0293183); a: A; b-d, f: L; e: US).

Mallotus species are predominantly dioecious, although a few species are dioecious or (rarely) monoecious, like in sect. Mallotus (e.g., M. barbatus Müll.Arg., M. macrostachyus (Miq.) Müll.Arg., M. mollissimus (Geiseler) Airy Shaw, M. paniculatus (Lam.) Müll.Arg.), sect.Rottleropsis s.1.(e.g., M. glomerulatus, M.hymenophyllus Airy Shaw, M.pierrei (Gagnep.) Airy Shaw) and sect. Polyadenii (e.g., M. polyadenos F. Muell.). 

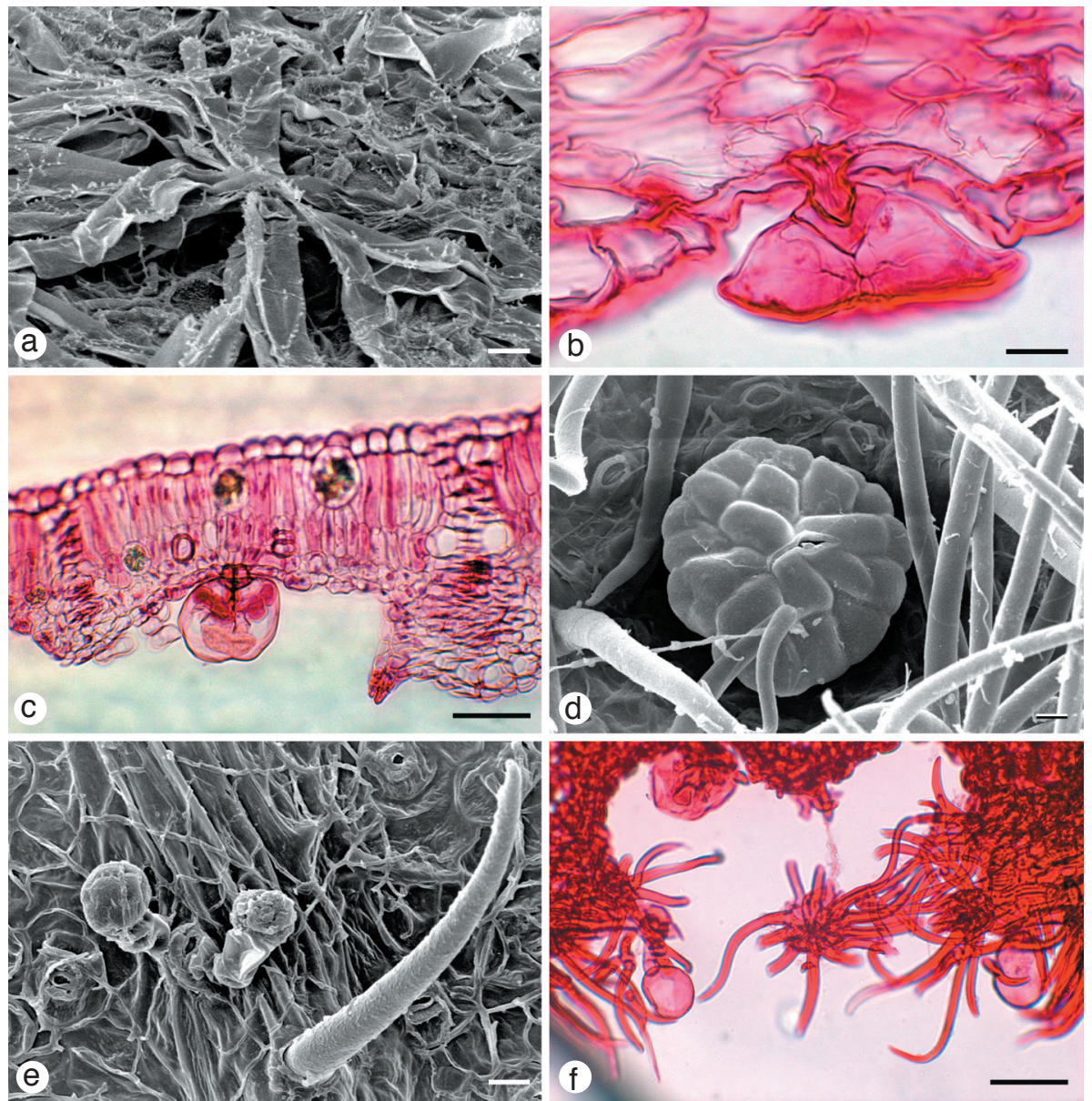

Fig. 2. Indumentum of Mallotus. a. Stellately-tufted hair of $M$. wrayi King ex Hook.f. (2-dimensional); b. disc-shaped glandular hair of M. polyadenos F. Muell.; c. globular-shaped glandular hair of M. paniculatus (Lam.) Müll.Arg.; d. globular-shaped glandular hair, with lobes, in M. repandus (Rottler) Müll.Arg.; e. capitate hairs of M. leptostachyus Hook.f.; f. capitate hair of M. tiliifolius (Blume) Müll.Arg. (a: S (E. Wright) 32300; b. P.I. Forster 27597; c: P.I. Forster 28767; d: J.E. Stocks s.n. (barcode L0436500); e: Kerr 12097A; f: Unknown s.n. (barcode L0293183); all L).

Almost $100 \%$ of all herbarium sheets are found to be either male or female plants. Therefore, the general interpretation is that dioecy is the common situation in these species. Exceptionally, sheets with both genders are found, these may be considered true exceptions or the species may actually be monoecious. The problem is that we are unable to check from dried material (only a small part of the plant) whether we are dealing with dioecy, dichogamy (both genders on the same plant, but separated by time), or occasionally monoecy. 
Indumentum - Plate $2 \mathrm{~d}$

The density of the indumentum is quantified by terms like dense, sparse, scattered (used instead of isolated) and glabrous, as defined by Hewson (1998). Leaf anatomical studies by Fišer et al. (in prep.) confirmed that the indument consists of non-glandular and glandular hairs. Among the non-glandular indument the most common hair types are simple, stellate and (stellately) tufted. Simple hairs are uni- (Fig. 1a) or multicellular (Fig. 1b). The first ones are the most frequent, while the latter have only been observed in M. philippensis (Lam.) Müll.Arg. and M. repandus (Rottler) Müll.Arg. Stellate hairs (Fig. 1c) have a short to long stalk $(14-150 \mu \mathrm{m})$, they are found in sect. Mallotus, and in some species of sect.Rottleropsis s.l. (e.g., M.tiliifolius (Blume) Müll. Arg., M.trinervius (K. Schum. \& Lauterb.) Pax \& K. Hoffm.). Tufted hairs (Fig. 1d, e) lack a stalk, they are in fact simple hairs inserted in a group. Some tufted hairs, when seen with the dissecting microscope, have the appearance of a stellate hair. They are radiating three-dimensionally (e.g., M. blumeanus Müll.Arg., M.tiliifolius), or in one plane, parallel to the blade surface (e.g., M. wrayi King ex Hook.f.) and are usually composed of 5 or more rays, more or less of the same length (Fig. 2a). To distinguish them from stellate hairs, we name them stellately-tufted hairs in the descriptions.

The glandular indument consists of globular to disc-shaped, and rarely capitate, hairs. Globular to disc-shaped hairs (Fig. 2b-d) are composed of a very short, multiseriate stalk $(5-11 \mu \mathrm{m})$, and a large, multicellular head (50-100 $\mu \mathrm{m}$ diam.), composed of 4 , to 8,16 or 32 glandular cells. They can be roundish or flattened, with or without ridges; and their colour varies from light yellow to dark red. Most species have globular to discshaped glandular hairs; however, they are absent in some of the species of sect. Rottleropsis s.l.(e.g., M.actinoneurus Airy Shaw, M.didymochryseus Airy Shaw, M.glomerulatus Welzen, M. lauterbachianus (Pax \& K. Hoffm.) Pax \& K. Hoffm., M. macularis Airy Shaw, M. mirus S.E.C. Sierra and M. monanthos Airy Shaw). Glandular hairs are also present in the sister genus Macaranga.

Capitate hairs (Fig. 2e, f) are composed of a long, uniseriate, multicellular stalk (20-40 $\mu \mathrm{m}$ ), and a unicellular head (c. $20 \mu \mathrm{m}$ diam.). The shape of the head suggests a glandular function; however, the content of the heads has not been studied (neither in the globular to disc-shaped hairs). Capitate hairs are rare in Mallotus and, in the sample of species studied so far, they have only been seen in M. leptostachyus Hook.f. and M. tiliifolius. Due to their size and colour (white to pale yellow), they are difficult to detect with a dissecting microscope.

Stipules - Plate $1 \mathrm{~b}$

The stipules in Mallotus are usually present, with the exception of a few species, e.g., M. chromocarpus, M. discolor F. Muell. ex Benth., M. nesophilus Müll.Arg., M. pleiogynus, M. tiliifolius. The shapes vary from deltoid to linear-triangular (sometimes obovate in $M$. cumingii), and they can be persistent to (early) caducous. In $M$. cauliflorus Merr. the size is much larger $(18-30$ by $8-10 \mathrm{~mm})$ when compared to the other Mallotus species $(0.3-14$ by $0.2-3.5 \mathrm{~mm})$.

Leaves

The petiole in Mallotus is rarely pulvinate; however, species of the Mallotus wrayi complex (M.caudatus, M.lancifolius Hook.f., M. spinifructus Welzen \& S.E.C. Sierra, 
M. wrayi) are recognized by the presence of an apically pulvinate petiole. Species of sect. Mallotus, like M. barbatus and M. macrostachyus reach petiole lengths of up to $40 \mathrm{~cm}$ (according to label information, even up to c. $70 \mathrm{~cm}$ ).

Leaf bases and apices are very polymorphic in Mallotus. However, for some species only one state is present and therefore the above mentioned characters are diagnostic. For instance, peltate leaf bases are common in sect. Mallotus and sect. Stylanthus, while cordate leaf bases can be found in M. hymenopyllus; cuneate in M. caudatus, M. lanceolatus (Gagnep.) Airy Shaw, M. mirus; shallowly attenuate in M.glomerulatus, M. monanthos; shallowly emarginate in M.actinoneurus, M. hispidospinosus Welzen \& Chayam. Caudate apices are found in M.actinoneurus, M. khasianus, M. monanthos and M. spinifructus.

Most species have opposite leaves (apically subopposite), the remainder have alternate ones. When opposite they are usually unequal in size, but equal in shape. However, unequally shaped opposite leaves are present in, e.g., M.concinnus, M. havilandii, M.miquelianus; M. brachythyrsus is the only species with one leaf reduced and stipuliform (like in Cordemoya sect. Diplochlamys (Müll.Arg.) S.E.C. Sierra, Kulju \& Welzen).

The venation is pinnate, triplinerved or palminerved, although species like M.peltatus (Geiseler) Müll.Arg. show all types. The nerves loop near or end in the margin; the veins are scalariform and the veinlets reticulate (although the terms vein and nerve are interchangeable and identical in meaning, we have adopted to use the word 'nerve' for second order venation, and 'veins' for tertiary and higher order venation, thus adopting the terminology that has been used in previous Mallotus revisional articles). The faint nerves near the apex, starting from the marginal sinus upwards, were excluded when giving the total nerve count. Mallotus cambodianus (Gagnep.) Airy Shaw, M. korthalsii Müll.Arg. and M. longinervis M. Aparicio never have more than 5 nerves per side, whereas $M$. cauliflorus, M. havilandii, M. miquelianus and M. monanthus have between 10-19 nerves per side.

The lower surface of the blade is usually characterized by the presence of glandular hairs. Sometimes they are few and/or difficult to find (e.g., M. calocarpus Airy Shaw, M. cumingii, M. hymenophyllus). The upper surface of the leaves rarely has glandular hairs, only few exceptions are found (e.g., sect. Polyadenii, M. cauliflorus, M. brachythyrsus and some species of sect. Stylanthus).

The species of sect. Stylanthus have a characteristic smell of fenugreek when dried, which is useful for identification purposes.

\section{Extrafloral nectaries}

Extrafloral nectaries are nectar producing organs found outside the flower. As they are not involved in pollination, their secretion is apparently related to attract ants (So, 2004). Mallotus has flattened extrafloral nectaries on the upper leaf surface (also present in the sister genus Macaranga). As in other Euphorbiaceae, they consist of an epidermis of palisade-like cells with compact mesophyll below (Zimmermann, 1932).

Extrafloral nectaries in Mallotus are orbicular to elliptic, and their size ranges from $0.2-3$ by $0.2-1 \mathrm{~mm}$. They occur along the margin, near the base, near the apex, along the midrib and/or all over the blade. Usually they are present and conspicuous; however, in M. attenuatus, M. coudercii and M. hispidospinosus, they are inconspicuous and sometimes even absent. 

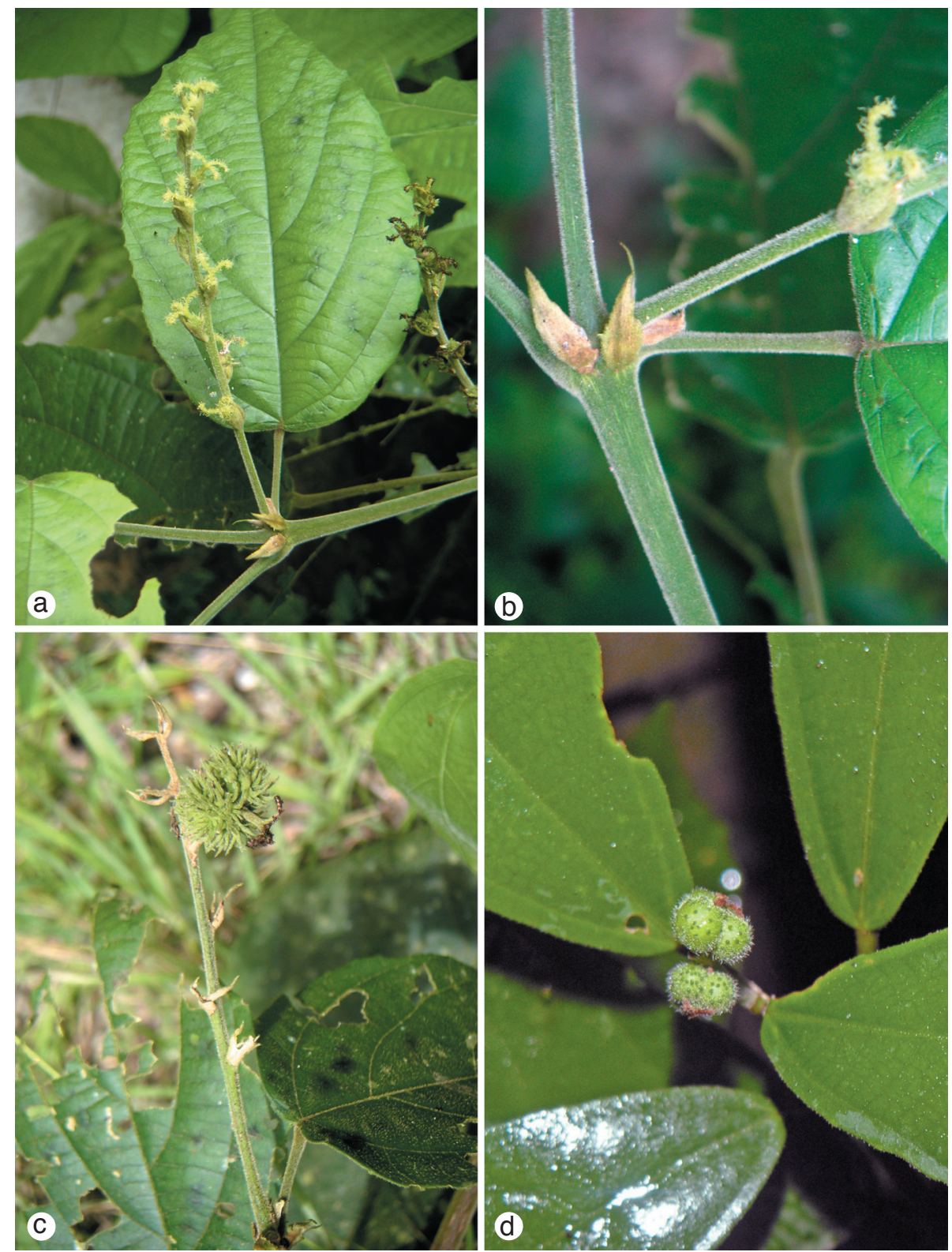

Plate 1. a-c: Mallotus leucocalyx Müll.Arg. a. Pistillate inflorescence; b. stipules; c. infructescence. - d: Mallotus decipiens Müll.Arg. Fruits. Photo S.E.C. Sierra. 

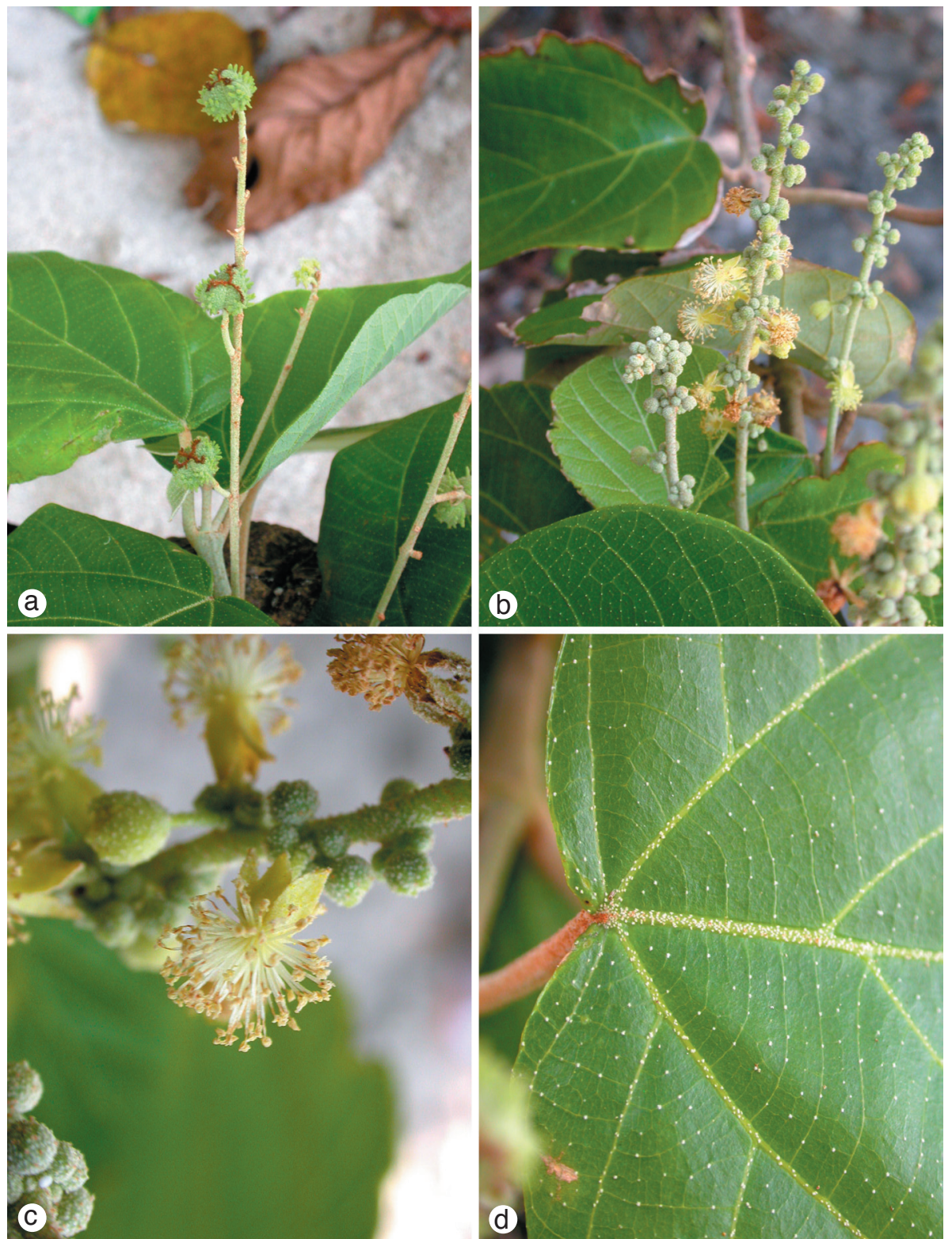

Plate 2. Mallotus tiliifolius (Blume) Müll.Arg. a. Infructescence; b. staminate inflorescences; c. staminate flower; d. leaf blade upper surface showing indumentum. Photo J.W.F. Slik. 


\section{Domatia}

When present, hair-tuft domatia are common in Mallotus; however, pocket domatia are sometimes also found (e.g., M.polyadenos, M. sumatranus (Miq.) Airy Shaw). In some species the domatia at the leaf base are conspicuous (densely hairy) and can even be seen with the naked eye (e.g., M. chromocarpus, M.floribundus (Blume) Müll.Arg., M. macrostachyus).

\section{Inflorescences - Plate 1a, 2b}

Inflorescences in Mallotus lack a terminal flower. They have bracts which subtend one flower or a fascicle of several flowers. The staminate inflorescences usually have several flowers per bract, the pistillate ones usually only one. A pedicel is usually present; however, in some cases the flowers are (sub) sessile. The fascicles usually grow along the entire axes with distinct internodes between bracts. However, in some cases fascicles are grouped at the apex, giving an umbel-like appearance. Additionally, the axes can be very reduced to almost absent, and then the flowers seemingly grow in glomerules.

The actual type of branching in the fascicles cannot be deduced, as the flowers are too closely packed to see which bract (if any) belongs to which flower. However, as it is generally believed that the inflorescences of Euphorbiaceae are essentially cymose or sympodial (Webster 1994: 10), the ultimate branching in Mallotus is supposed to be sympodial as well. The inflorescences would then be thyrsoid (main branching racemose, ultimate branching cymose).

Radcliffe-Smith (2001) mentions that the inflorescences are spiciform, racemiform, paniculate or thyrsiform. Because no inflorescence developmental studies in Mallotus have been conducted, to simplify practical terminology, authors working in the revision of Mallotus have called the inflorescences racemes (non-branching, flowers pedicelled), spikes (idem, flowers sessile) or panicles (branching); umbel-like (pseudo-umbels) is used when the internodes are short and several flowers are originating from one bract; the term glomerules is used when no axes are visible.

Examples of panicles are M. mollissimus, M. paniculatus, M. tetracoccus Kurz; of racemes are $M$. philippensis, $M$. repandus; of reduced racemes is $M$. calocarpus; of spikes is $M$. macrostachyus; of umbel-like is $M$. claoxyloides, $M$. ficifolius $\mathrm{Pax} \&$ $\mathrm{K}$. Hoffm.; of glomerules is M. glomerulatus.

\section{Flowers - Plate 2c}

Flowers in Mallotus are small and do not exceed $10 \mathrm{~mm}$ diam., they are usually pedicellate and the pedicel sometimes has a distinct abscission zone. Petals are absent, while the sepals are always present and often persistent (in the female flowers of few species, such as $M$. cumingii and $M$. peltatus, the spathaceous calyx is early caducous).

Staminate flowers have strong differences in diameter, small, up to c. $2.5 \mathrm{~mm}$ (e.g., M. fuscescens (Thwaites) Müll.Arg., M. longinervis, M. puber Bollendorff), or large, up to c. $9 \mathrm{~mm}$ (e.g., M. barbatus, M. nudiflorus). Interstaminal disc-glands are small and inconspicuous, and in few cases present (e.g., M. cumingii, M. khasianus, M. minimifructus S.E.C. Sierra, M. wrayi). Mallotus can have up to c. 130 stamens (M. apelta Müll.Arg., M. tiliifolius), the filaments are free, rarely connate (M. connatus M. Aparicio, M. eximius Airy Shaw, M. monanthos, M. subcuneatus), glabrous or sometimes hairy. The connectives have a papillose appearance, they are variable in 

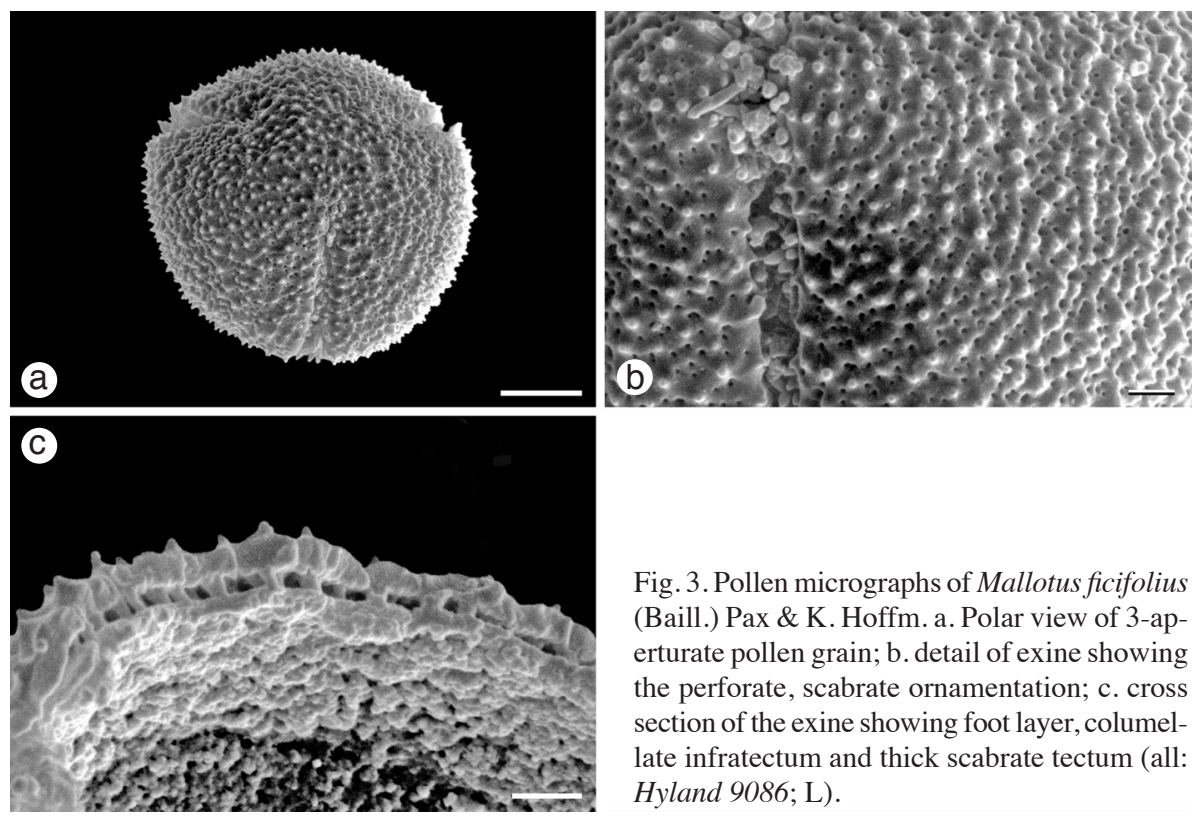

Fig. 3. Pollen micrographs of Mallotus ficifolius (Baill.) Pax \& K. Hoffm. a. Polar view of 3-aperturate pollen grain; b. detail of exine showing the perforate, scabrate ornamentation; c. cross section of the exine showing foot layer, columellate infratectum and thick scabrate tectum (all: Hyland 9086; L).

size, sometimes even within one species, in most cases they are narrow to distinctly broadened, to irregularly apiculate. In a few species they have an umbrella-like appearance (e.g., M. blumeanus, M. cauliflorus, M. chromocarpus, M.pleiogynus, M. sphaerocarpus (Miq.) Müll.Arg.). Pistillodes are rarely present (e.g., M. resinosus (Blanco) Merr., M. tiliifolius, M. trinervius).

The diameter of the pistillate flowers is also variable, the smallest (c. $1 \mathrm{~mm}$ ) is found in M. decipiens Müll.Arg. and the largest (c. $9 \mathrm{~mm}$ ) in M. caudatus. The number of locules ranges from 1-9; however, 2- or 3-locular ovaries are usual. The styles are usually present, stigmas are simple, erect to reflexed, and on the upper surface they are papillose or plumose.

\section{Pollen}

Mallotus pollen is medium-sized (Polar axis by Equatorial diameter $=16-28.5$ by $18-30.6 \mu \mathrm{m})$ and uniform in shape and ornamentation. The aperture system is (2- or) 3- (or 4-)colporate. The exine ornamentation is perforate/microreticulate with scabrae, the foot layer is thin, the columellae are short and the tectum is continuous and thick (Fig. 3a-c). Observations were made based on a sample of 37 species from different sections. For additional information see Nowicke \& Takahashi (2002) and Van Welzen et al. (2004).

Fruits - Plate 1c, 1d, 2a

The fruits are mostly dehiscent to tardily dehiscent capsules, rarely indehiscent, drupaceous and variously fleshy (e.g., M. blumeanus, M. cumingii, M. nudiflorus, M. sphaerocarpus). The capsules open in various ways, septicidally-loculicidally (first dehiscence along septa followed by dehiscence over the locule) to loculicidallysepticidally or rarely only loculicidally (e.g., M. didymochryseus, M.polycarpus). Their 
shape is oblate to sometimes globose, shallowly to deeply lobed and they rarely have wings (some species of sect. Polyadenii) or longitudinal ridges (e.g., M. pleiogynus). The surface is smooth to spiny (to somewhat rugose to verrucose), hairy or glabrous and sometimes densely covered with glandular hairs (e.g., M. chromocarpus, M. discolor, $M$. nesophilus, $M$. pleiogynus). The sizes of the fruits differ considerably, from 3-4 by $4-6 \mathrm{~mm}$ in $M$. decipiens, to $18-29$ by $21-35 \mathrm{~mm}$ in $M$. nudiflorus. The seeds are more or less globose, rarely triangular in cross section (M. nudiflorus) or lenticular (M. blumeanus, M. sphaerocarpus).

Sarcotestas and fleshy endocarps are rarely present in Mallotus. Seed development studies in M. nudiflorus by Dwivedi (1994) indicate that a sarcotesta is present and that the mature seed derives its dark brown colour from the contents of the outer layer. Kulju et al. (2007) indicate that M. polycarpus has a sarcotesta. Kurz (1877: 396) describe the seeds of M. leucocarpus as being "enveloped in a nearly complete snow-white sappy arillus", probably a sarcotesta.

In sect. Mallotus a fleshy layer is present around the seed when these are young; this layer is absent in mature fruits. Mallotus sphaerocarpus has a thick, fleshy, whitish endocarp, which encapsulates the seeds.

\section{Distribution}

Mallotus has a palaeotropical distribution, from West (Senegal to Liberia) to East (Ethiopia to Tanzania) Africa to West Madagascar, Pakistan, Sri Lanka, India, Bangladesh, Myanmar and Nepal, South China, Korea, South Japan, throughout Southeast Asia and Malesia to East Australia and the West Pacific (Solomon Islands, Republic of Palau, Federated States of Micronesia, New Caledonia and Fiji). The genus occurs mainly in (sub)tropical Asia and the Pacific (c. 110 spp.), with only two species in tropical Africa and Madagascar (M. oppositifolius (Geiseler) Müll.Arg. and M. subulatus Müll.Arg.).

In the Malesian region 52 species are recognized in total, viz. 4 in sect. Mallotus, 2 in sect. Philippinensis, 4 in sect. Polyadenii, 34 in sect. Rottleropsis s.1., 3 in sect. Stylanthus and 5 excluded species from Cordemoya sect. Hancea.

Some species in Mallotus are widespread (e.g., M.paniculatus, M.peltatus, M.philippensis, $M$.repandus, $M$.resinosus, $M$. tiliifolius), whereas others have restricted ranges (e.g., M.atrovirens Wall. ex Müll.Arg., M.cauliflorus, M.chromocarpus, M. sphaerocarpus)

Mallotus leucodermis has a disjunct distribution, occurring in West Malesia (Sumatra, Peninsular Malaysia, Borneo) and Irian Jaya (Bollendorff et al., 2000). Other frequently collected species, with a widespread distribution throughout Southeast Asia and large numbers of collections, are absent in certain areas (e.g., M. repandus ${ }^{1}$ not in Borneo, M. resinosus not in Peninsular Malaysia, Sumatra and a large part of Borneo).

1) In the revision of sect. Philippinensis (Sierra et al., 2005), the distribution map of. M. repandus shows only one collection occurring in Borneo (based on Kostermans s.n., L0436500). However, further inspection revealed that this collection has two labels. One is a field label with the locality "Ujung Kulon" (Java). The other one is a typed label with the locality "Berau near Tgd. Redeb, E Indonesia, Borneo". We think that the collecting locality is more likely to be in Java. The same case occurs with a collection of M. rufidulus (Miq.) Müll.Arg. by Kostermans s.n. (barcode L0294643). 
Ecology

Mallotus shows a large variety of life-history strategies, some species being early successional pioneers, while others are climax species. Mallotus species occur in different habitats, like the understorey of primary forest, semi-evergreen vine-thickets, mangroves, notophyll and microphyll vine-forest, or in disturbed secondary forest (selectively logged, fire damaged, abandoned after shifting-cultivation), preferring open places like river banks, forest edges, road sides, cleared areas; in wet (riverine, swampy) and periodically inundated areas, and on well-drained soil. They occur from sea level up to $2100 \mathrm{~m}$ altitude, on a large variety of soil types, like thick humus, limestone, sandstone, sandy clay, sandy loam, ultramafic, volcanic rock, gravel, red shale.

Mallotus and Macaranga are indicators of forest disturbance (Slik et al., 2003). Slik (2005) developed a methodology to assess the type of forest disturbance in lowland tropical forest in Southeast Asia, in particular useful when ecological field data are absent. For this purpose, ecological attributes in both genera were used together with three morphological characters related to the successional life history strategy of the species (wood density, seed size and leaf shape).

\section{Pollinators and animal interaction}

Hardly any information exists about how Mallotus interacts with animals. Two genera of bees, Trigona sp. and Apis dorsata, visit the staminate flowers of M. brevipetiolatus Gage. Corlett (2001) stated that in M.paniculatus only male plants are visited by pollen collecting bees, suggesting that the pollen transfer must be by wind. Label information from herbarium collections revealed the presence of thrips visiting M. apelta. Moog et al. (2002) provided evidence for thrips pollination in the sister genus Macaranga. They found that Macaranga hullettii King ex Hook.f. is pollinated predominantly by one thrips species, Neoheegeria sp. (Phlaeothripidae). Adults and larvae were observed feeding on the adaxial base of the bracteoles.

Studies on stick insects (Phasmida) by Blüthgen et al. (2006) in tropical ecosystems revealed that Asceles margaritatus had a largely restricted diet, consuming preferably M. miquelianus leaves, rather than other plants.

\section{Fruit and seed dispersal}

Most fruits are dry, explosive capsules, facilitating seed dispersal; some species also have interactions with animals. According to Esser (2003) spiny, explosive capsules could indicate epizoochory, but could also be interpreted as protection against herbivory.

Forster (1999) stated that birds play an important role in the seed dispersal of Mallotus due to the bright colour of the fruits and the relatively large seeds. Sato \& Sakai (2005) studied the dispersal of M. japonicus Müll.Arg. by observing the foraging behaviour of frugivorous bird species in a warm-temperate coniferous plantation, and concluded that four species of Muscicapidae (Cyanoptila cyanomelana, Ficedula narcissina, Muscicapa sibirica, M. griseisticta) were effective seed dispersers of Mallotus japonicus because of their high frequency of seed foraging.

Dinerstein \& Wemmer (1988) mention that M. nudiflorus is a common tree in the riverine forests of Nepal and its fruits are dispersed by rhinoceros and domestic cattle. 
Uses

The roots of Mallotus are used against muscle stiffness ( $M$. barbatus), fever ( $M$. minimifructus), headaches and malaria (M.mollissimus), gout and rheumatic afflictions (M. nudiflorus) and for dissolving coagulated blood and contusions (M.philippensis).

The bark (M.philippensis) is active against gram-positive and gram-negative bacteria. The wood is used for hedges and timber (M. tiliifolius), building construction (M. mollissimus) and firewood (M. caudatus, M. khasianus).

The leaves are used for Staphylococcus aureus infections (M. mollissimus), applied for splenomegaly (M.minimifructus), used as fodder (M.nudiflorus, M. philippensis), and as an antidote against snake-poison (M. macrostachyus).

The glandular hairs of the fruits provide a red or orange dye (M. philippensis, M. discolor) and have an antihelmintic activity mainly attributed to the phloroglucinol derivates rottlerin and isorottlerin. The oil of the seeds is used for making candles (M. barbatus, M. philippensis) or as a substitute for tung oil (obtained from Aleurites spp.) in the production of paint and varnish.

The flowers $M$. floribundus are fragrant and used to flavour food and in decorations.

Only two species have been reported to be cultivated as ornamentals (M.philippensis and $M$. floribundus). In few cases the plants are used as living fences (M. oppositifolius).

After Thin \& On, 1998; Bollendorff et al., 2000; Slik \& Van Welzen, 2001a; Lugt, 2003; Sierra \& Van Welzen, 2005; Sierra et al., 2005; Van Welzen \& Sierra, 2006; Kulju et al., 2007.

\section{MALLOTUS section ROTTLEROPSIS s.l.}

Mallotus Lour. sect. Rottleropsis Müll.Arg. in DC. (1866) 957; Airy Shaw (1968) 379; (1972) 301. - Type species: Mallotus lappaceus Wall. ex Müll.Arg., see note 1.

Echinocroton F. Muell. (1858) 31. - Mallotus Lour. sect. Echinocroton (F. Muell.) Pax \& K. Hoffm. (1914) 148; Airy Shaw (1966) 41. - Type species: Echinocroton claoxyloides F. Muell. (= Mallotus claoxyloides (F. Muell.) Müll.Arg.).

Plagianthera Rchb.f. \& Zoll. (1856) 19. - Mallotus Lour. sect. Plagianthera (Rchb.f. \& Zoll.) Pax \& K. Hoffm. (1914) 156. - Type species: Plagianthera oppositifolia Rchb.f. \& Zoll. (= Mallotus blumeanus Müll.Arg., non Mallotus oppositifolius (Geiseler) Müll.Arg.).

Mallotus Lour. sect. Axenfeldia Pax \& K. Hoffm. (1914) 187; Airy Shaw (1968) 393; (1972) 293. - Type species: Axenfeldia intermedia Baill. (= Mallotus intermedius (Baill.) N.P. Balakr.).

Shrubs to trees, dioecious or rarely monoecious. Indumentum composed of simple, tufted, stellately-tufted ( $\geq 5$ rays, radially symmetrical), rarely peltate hairs, and usually globular to disc-shaped glandular hairs, rarely capitate glandular hairs. Stipules usually present. Leaves opposite, unequal in size, rarely also alternate; blade base rarely peltate; margin entire to crenate to dentate, often with minute glandular teeth, upper surface usually with generally conspicuous extrafloral nectaries, sometimes with glandular hairs, lower surface usually with glandular hairs, hair tuft domatia sometimes present, venation pinnate, triplinerved or palminerved, nerves looping or ending in margin. Inflorescences axillary or terminal, rarely ramiflorous or cauliflorous, unisexual, rarely bisexual; bracts 1 (to several when inflorescence umbel-like) per node, bracteoles sometimes present. Flowers not exceeding $10 \mathrm{~mm}$ diam.; sepals free or connate, usually persistent; petals absent. Staminate inflorescences racemes, rarely glomerules, 
with 1-15 (to rarely more) flowers per bract/node. Staminate flowers: sepals (2-)3-5 (-6); disc-glands rarely present, 3-16; stamens 20-130, filaments free or connate; pistillode often present. Pistillate inflorescences racemes, umbel-like, or sometimes reduced to a terminal flower; with 1 to several (when umbel-like) flowers per bract. Pistillate flowers: sepals (2-)3-6; staminodes rarely present; ovary (1-)2-4(-5)locular, 1 ovule per locule; style usually present; stigmas (erect to) reflexed, (papillose or) plumose adaxially. Fruits dehiscent to tardily dehiscent capsules, or indehiscent, drupaceous and variously fleshy, oblate (to globose), usually lobed, surface smooth (to somewhat rugose to verrucose) to spiny, glandular and/or hairy (to glabrous), opening septicidally-loculicidally to sometimes loculicidally-septicidally, rarely only loculicidally; wall rarely with a thick, fleshy, whitish endocarp; column usually present. Seeds \pm globose, rarely lenticular or triangular in cross section, surface smooth to rugose; rarely covered with a thin sarcotesta.

Distribution - From SW (Senegal to Liberia) to SE (Ethiopia to Tanzania) Africa to W Madagascar, Sri Lanka, India, Myanmar and Nepal, S China, throughout SE Asia and Malesia to E Australia and the W Pacific (Solomon Islands, Republic of Palau, Federated States of Micronesia and Fiji).

Notes - 1. Wallich 7738 and 7845 from Myanmar are mentioned in the protologue of M. lappaceus. Although we have only been able to study them from digital pictures sent by K, their appearance and description by Müller Argoviensis (1866) agree with the concept of sect. Rottleropsis s.l. The collections have long stipules and bracts, which are features that are also present in M. dispar and M. leucocalyx.

2. Following Govaerts et al. (2000) and recent publications of new species of Mallotus, we estimate that c. 30 names have been published for non-Malesian taxa (not treated in this study), which might also belong to sect. Rottleropsis s.l.

\section{IDENTIFICATION KEYS}

\section{Notes and recommendations}

The species of sect. Rottleropsis s.l. are separated by a specific combination of characters, which makes the construction of a dichotomous key difficult, especially when there are no clear distinctive vegetative characters which can be used within two leads. For this reason two types of keys, regional dichotomous and a synoptical key, are presented here.

The regional keys are split into five areas: I. Africa and Madagascar; II. India, Sri Lanka, Mainland Asia; III. Andaman \& Nicobar Islands, Taiwan, Peninsular Thailand to Borneo; IV. Philippines and Central Malesia; and V. New Guinea, Australia to Fiji. Endemic species occurring outside Malesia, Thailand or Africa are not revised here, and are therefore not included in the keys; this means that the regional keys II and V do not include all the species found in India, Sri Lanka, Mainland Asia, or Australia, respectively.

When present, glandular hairs can be easily detected on the lower leaf surface with a dissecting microscope. When extrafloral nectaries are mentioned, the larger ones are meant; therefore, the minute, orbicular, extrafloral nectaries which are sometimes present on the upper half of the leaf should not be taken into account.

The seven species indicated with characters A-G are described in two other publications (see Van Welzen \& Sierra, 2006; Kulju et al., 2007). 


\section{REGIONAL KEYS}

\section{Key to the species of Africa and Madagascar}

1a. Stipules 1.7-4 mm long. Leaf blades broadly ovate to ovate, lower surface densely covered with glandular hairs. Pistillate inflorescences with 8-62 nodes per branch. Fruits surface smooth or rarely scatteredly verrucose. - Africa, Madagascar....

30. M. oppositifolius

b. Stipules 3.5-12 $\mathrm{mm}$ long. Leaf blades ovate to elliptic, lower surface scatteredly covered with glandular hairs. Pistillate inflorescences with 1-3 nodes per branch.

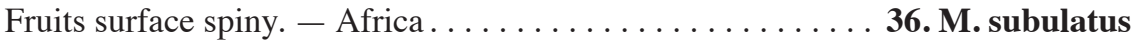

\section{Key to the species of India, Sri Lanka, Nepal, Bhutan, Bangladesh, Myanmar, China, Laos, Vietnam, Cambodia, Thailand (excl. Peninsular)}

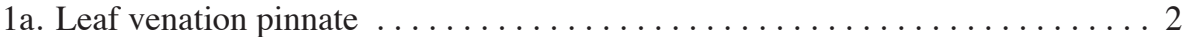

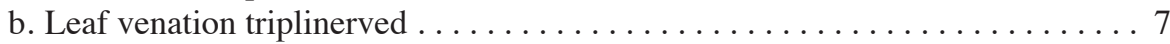

2a. Leaf blades ovate to elliptic, membranaceous, base shallowly emarginate, extrafloral nectaries rarely present, inconspicuous. Staminate filaments $0.5-1.2 \mathrm{~mm}$ long. Fruit hairs densely hairy . . . . . . . hispidospinosus

b. Leaf blades elliptic to obovate, chartaceous, base shallowly emarginate, obtuse, cuneate to shallowly attenuate, extrafloral nectaries always present, conspicuous. Staminate filaments $1-4.5 \mathrm{~mm}$ long. Fruit hairs absent to present . . . . . . 3

3a. Glandular hairs on leaf blades: present on upper surface, densely (to sparsely) present on lower surface. Glandular hairs on fruits densely to sparsely present . .

32. M. resinosus

b. Glandular hairs on leaf blades: absent on upper surface, absent to scatteredly present on lower surface. Glandular hairs on fruits absent to scatteredly present . . . . . 4

4a. Leaf blades: glandular hairs present on lower surface, domatia sometimes present. Staminate inflorescences 7-53 cm long, with 3-8 flowers per bract; flowers: pedicels 5-15 mm long. Pistillate inflorescences racemes, 20-45 cm long. Fruits spiny. - E India, Myanmar to China, Thailand, Cambodia . . . 19. M. khasianus

b. Leaf blades: glandular hairs absent or rarely present on lower surface, domatia absent. Staminate inflorescences (unknown for M. mirus) $1-2.3 \mathrm{~cm}$ long, with 1 flower per bract; flowers: pedicels 3-5 mm long. Pistillate inflorescences reduced to 1 terminal flower, $1-1.7 \mathrm{~cm}$ long. Fruits smooth. - Thailand . . . . . . . 5

5a. Stipules $2-3.5 \mathrm{~mm}$ long, early caducous. Petioles $6-15$ by $2.8-3.5 \mathrm{~mm}$. Leaf blades elliptic, coriaceous, upper surface glabrous on midrib and nerves. Fruits $9-10$ by $13-14 \mathrm{~mm} \ldots \ldots \ldots \ldots \ldots \ldots \ldots \ldots \ldots \ldots \ldots$ 16. M. glomerulatus

b. Stipules $5-6.5 \mathrm{~mm}$ long, persistent to caducous. Petioles $4-33$ by $1-1.8 \mathrm{~mm}$. Leaf blades elliptic to obovate, chartaceous, upper surface hairy on midrib and nerves. Fruits $10-17$ by $11-22 \mathrm{~mm} \ldots \ldots \ldots \ldots \ldots \ldots \ldots \ldots \ldots \ldots \ldots \ldots \ldots \ldots \ldots$

6a. Leaf base cuneate. Stipules, petioles and fruits scatteredly hairy or absent. Tufted hairs appressed. - Thailand, Prachin Buri Province ........ 27. M. mirus

b. Leaf base attenuate. Stipules, petioles and fruits densely hairy. Tufted hairs erect. - Thailand, Chanthaburi Province ............ 5. M. calocarpus 
7a. Extrafloral nectaries absent to rarely present, inconspicuous . . 9. M. coudercii

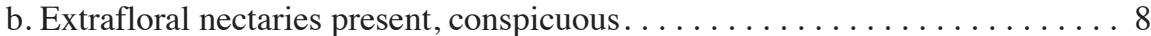

8a. Stipules $2-4 \mathrm{~mm}$ wide . . . . . . . . . leucocalyx

b. Stipules $0.3-1.8 \mathrm{~mm}$ wide (unknown for M. polycarpus) . . . . . . . . 9

9a. Leaf blades: upper surface rough, broadly ovate to elliptic. Filaments hairy. Pistillate bracts entire or trilobed $\ldots \ldots \ldots \ldots \ldots \ldots \ldots \ldots$ 31. M. pierrei

b. Leaf blades: upper surface smooth, ovate to obovate. Filaments glabrous or hairy.

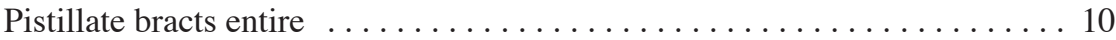

10a. Stipules 3-10 mm long. Leaves ovate, narrowly elliptic (to obovate), chartaceous. Filaments glabrous or hairy. Pistillate inflorescences umbel-like or racemes. Fruits

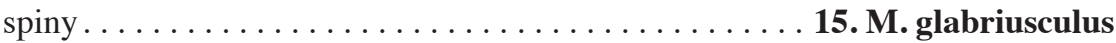

b. Stipules 1.8-5.2 mm long (unknown for M.polycarpus). Leaves ovate to obovate, membranaceous to chartaceous. Filaments glabrous. Pistillate inflorescences

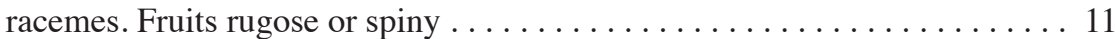

11a. Petioles 2-24 mm long. Glandular hairs always present. Staminate inflorescences up to $16 \mathrm{~cm}$ long; flowers $2.5-4 \mathrm{~mm}$ diameter. Stigmas $0.7-2.5 \mathrm{~mm}$ long. Fruits

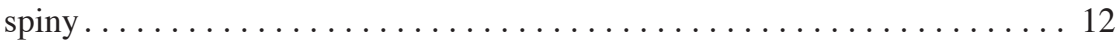

b. Petioles 18-120 mm long. Glandular hairs sometimes absent. Staminate inflorescences up to $30 \mathrm{~cm}$ long; flowers 4.7-9 $\mathrm{mm}$ diam. (unknown for M. polycarpus).

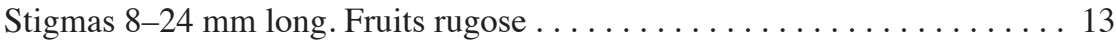

12a. Extrafloral nectaries 3-7 per side. Leaves apex acute to acuminate. Staminate inflorescences with 10-15 nodes per branch; flowers: stamens 30-60. Pistillate inflorescences 4-8 cm long; flowers: stigma 1.2-2.5 mm long. Fruits 6-8.8 mm wide. - Thailand, Cambodia, Laos, Vietnam, S China . . . 21. M. lanceolatus

b. Extrafloral nectaries 1 or 2 per side. Leaves apex cuspidate to caudate. Staminate inflorescences with 22-41 nodes per branch; flowers: stamens 22-32. Pistillate inflorescences 6.8-16 cm long; flowers: stigma 0.7-1 mm long. Fruits 4-6 mm wide. - Bangladesh, Myanmar, Laos, Vietnam, Thailand, Peninsular Malaysia

11. M. decipiens

13a. Leaf blades length/width ratio $1-2.3$ (-4.8 in immature leaves), base cordate to obtuse (to acute to cuneate especially in immature leaves), domatia sometimes present. Pistillate inflorescences $1.5-10.5 \mathrm{~cm}$ long; flowers with 3-5 locules. Fruits drupes, wall 2-8 mm thick. - From India and Nepal throughout mainland SE Asia to southern China and W Malesia . . . . . . . . . . . . . .

D. M. nudiflorus (see Kulju et al., 2007)

b. Leaf blades length/width ratio 1-1.7, base cordate to truncate, domatia absent. Pistillate inflorescences 3-5.5 cm long; flowers with 2 locules. Fruits capsules, wall 0.25-1 mm thick. - India . . . .... M. polycarpus (see Kulju et al., 2007)

III. Key to the species of the Andaman \& Nicobar Islands, Taiwan, Peninsular Thailand, Peninsular Malaysia, Sumatra, Java, Borneo

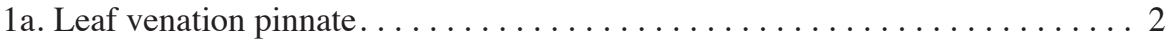

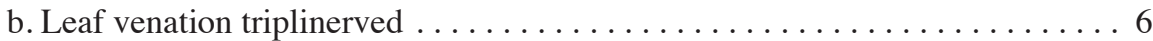

2a. Stipules 9-13 mm long. Leaf blades: narrowly obovate, glandular hairs absent.

- Peninsular Malaysia ... . . . . . . . . . . . . . . . . . 3 
b. Stipules 1.8-6 mm long. Leaf blades: ovate to obovate, glandular hairs sometimes

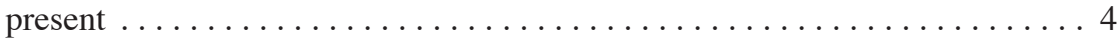

3a. Stipules 2.5-3.5 mm wide, persistent. Petioles 14-61 mm long. Leaf blades: base shallowly emarginate, nerves 9 or 10 per side ...... 1. M. actinoneurus

b. Stipules 1.5-2 mm wide, caducous. Petioles 5-15 mm long. Leaf blades: base attenuate, nerves $15-18$ per side . . . . . . . . . . monanthos

4a. Petioles 4-118 mm long. Leaf blades with 4-11 nerves per side. Pistillate flowers: calyx entirely connate, early caducous, ovary 1- (or 2-)locular, stigma 1 . Fruits drupes, surface smooth to slightly verrucose

B. M. cumingii (see Kulju et al., 2007)

b. Petioles $2-20(-35) \mathrm{mm}$ long. Leaf blades with $8-16$ nerves per side. Pistillate flowers: calyx basally connate, persistent, ovary 3-locular, stigmas 3 . Fruits cap-

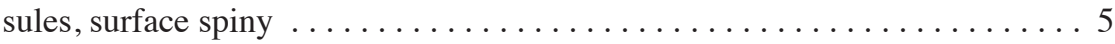

5a. Petioles 2-6 mm long. Leaf blades: base cuneate to shallowly emarginate. Staminate inflorescences: nodes per branch 9-68; flowers: sepals 2-3 mm long. Pistillate flowers 2.5-3 mm diam., pedicels 1-3 mm long. - Thailand, Peninsular

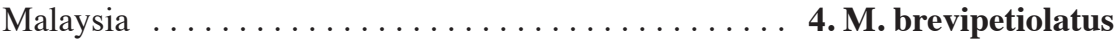

b. Petioles 2.5-20(-35) mm long. Leaf blades: base obtuse, cuneate to shallowly attenuate. Staminate inflorescences: nodes per branch 7-39; flowers: sepals 1.5-2.3 $\mathrm{mm}$ long. Pistillate flowers $1.5-2 \mathrm{~mm}$ diam., pedicels $0.5-1 \mathrm{~mm}$ long. - India to Sri Lanka, Andaman \& Nicobar Islands, Thailand, Laos, Vietnam, Philippines, Borneo, Java, Lesser Sunda Islands, Sulawesi, Moluccas, Papua New Guinea, Australia ....................... 32. M. resinosus

6a. Petioles apically pulvinate. Domatia always present $\ldots \ldots \ldots \ldots \ldots \ldots$ A. M. caudatus, C. M. lancifolius, F. M. spinifructus, G.M. wrayi $\ldots \ldots \ldots \ldots \ldots \ldots . \ldots \ldots$ (see Van Welzen \& Sierra, 2006)

b. Petioles apically not pulvinate. Domatia sometimes present $\ldots \ldots \ldots \ldots 7$

7a. Leaf base cordate. Domatia always present. - Thailand .18. M. hymenophyllus

b. Leaf base truncate to emarginate. Domatia sometimes present . . . . . . . 8

8a. Indumentum only with stellately-tufted hairs. Fruits indehiscent, $10-19$ by $4-19$ $\mathrm{mm}$, surface smooth or with slightly verrucose to spiny-like projections; wall

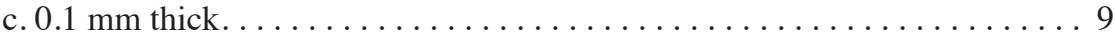

b. Indumentum with simple, tufted and/or stellately-tufted hairs. Fruits dehiscent or indehiscent, if indehiscent then $18-29$ by $21-35 \mathrm{~mm}$, surface rugose, wall $2-8$

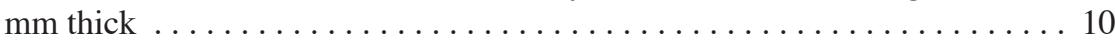

9a. Indumentum of appressed, brown-yellow hairs. Pistillate flowers 3-4 mm diam., pedicels $1.3-2 \mathrm{~mm}$ long. Fruits ellipsoid, $10-12$ by $4-4.4 \mathrm{~mm}$, without ridges when dry, surface smooth, sparsely hairy. - Sumatra, Java . 3. M. blumeanus

b. Indumentum of erect, yellow hairs. Pistillate flowers $0.8-1.2 \mathrm{~mm}$ diam., pedicels $0.8-1.2 \mathrm{~mm}$ long. Fruits spheroid, $15-19$ by $15-19 \mathrm{~mm}$, with 3 longitudinal ridges when dry, surface slightly verrucose, densely hairy. - Sumatra

34. M. sphaerocarpus

10a. Leaf blades: lower surface densely hairy, broadly ovate to ovate, domatia absent.

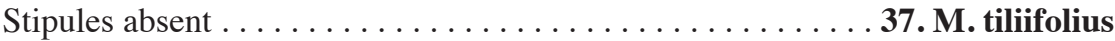

b. Leaf blades: lower surface sparsely to scatteredly hairy, broadly ovate to obovate, domatia sometimes present. Stipules present $\ldots \ldots \ldots \ldots \ldots \ldots$ 
11a. Leaf blades: elliptic, nerves 2 or 3 per side. Stipules deltoid, $0.4-0.5$ by $0.4-0.5$

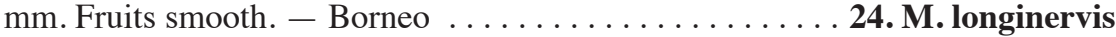

b. Leaf blades: ovate to obovate, nerves 3-10 per side. Stipules triangular to linear-

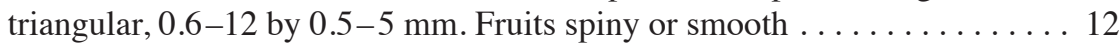

12a. Stipules $0.6-1.3 \mathrm{~mm}$ long. Fruits smooth capsules . . . . . 29. M. montanus

b. Stipules 1.5-12 mm long. Fruits spiny capsules or rugose drupes . . . . . . 13

13a. Extrafloral nectaries marginal throughout blade, sometimes also all over the

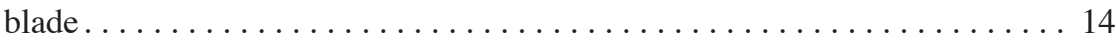

b. Extrafloral nectaries basal or marginal on lower half, never all over the blade 17

14a. Leaf blades with 4-6(-9) nerves per side. Stipules $0.5-1 \mathrm{~mm}$ wide . . . . . 15

b. Leaf blades with 7-9 nerves per side. Stipules $1.5-5 \mathrm{~mm}$ wide . . . . . . . 16 15a. Leaf blades drying green to green-brown. Stipules linear-triangular. Pistillate inflorescences with entire or trilobed bracts; pistillate flowers: buds ellipsoid, style 2-4 mm long, stigmas $0.8-1 \mathrm{~mm}$ wide. Staminate flowers with connate filaments.

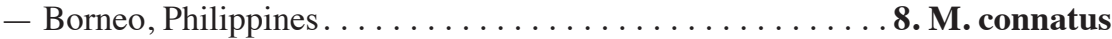

b. Leaf blades drying brown. Stipules narrowly triangular. Pistillate inflorescences with trilobed bracts; pistillate flowers: buds ovoid, style $0-1(-2) \mathrm{mm}$ long, stigmas 1-1.3 mm wide. Staminate flowers with free to slightly connate at the base. - Sumatra, Java, Borneo, Lesser Sunda Islands. . . . . . . . . 33. M. rufidulus

16a. Stipules scatteredly hairy to glabrous outside. Petioles $1.3-1.8 \mathrm{~mm}$ wide. Leaf blades length/width ratio 1.5-1.9. Staminate flowers 3-4.2 mm diam., filaments free, glabrous. Pistillate inflorescences 9.4-16 cm long; flowers: pedicels 1-2 $\mathrm{mm}$ long. Fruits 7-7.5 mm long. - Myanmar, Thailand, Peninsular Malaysia, Sumatra, Java, Borneo (Sabah), Philippines, Sulawesi . . . . 23. M. leucocalyx

b. Stipules densely hairy outside. Petioles $2-3 \mathrm{~mm}$ wide. Leaf blades length/width ratio 1.7-2.6. Staminate flowers 4.8-5.2 $\mathrm{mm}$ diam., filaments connate in lower half, hairy. Pistillate inflorescences $15-34 \mathrm{~cm}$ long; flowers: pedicels 6-11 mm long. Fruits 17-20 mm long. - Peninsular Malaysia, NW Borneo . . 14. M. eximius

17a. Extrafloral nectaries 1 or 2 per side, at least one pair always present on the first pair of nerves . . . . . . . . . . . . . . . . . . 18

b. Extrafloral nectaries 1-7(-17) per side, always below the nerves . . . . . . 19

18a. Stipules 2-4 mm long. Leaf blades obovate. Inflorescences with triangular bracts. Staminate flowers: pedicels 1.8 -2.2 mm long, stamens 22-32. Pistillate flowers: stigmas 0.7-1 mm long. Fruits 3-4 mm long. - Bangladesh, Myanmar, Laos, Vietnam, Thailand, Peninsular Malaysia . . . . . . . . 11. M. decipiens

b. Stipules 5-6 mm long. Leaf blades ovate to obovate. Inflorescences with narrowly triangular to linear-triangular bracts. Staminate flowers: pedicels 2-4 $\mathrm{mm}$ long, stamens 28-50. Pistillate flowers: stigmas 3.5-6 mm long. Fruits 8-12 mm long. - Thailand, Peninsular Malaysia, Sumatra, Java . . . . . . . 13. M. dispar

19a. Leaf blades: first pair of nerves ending in the upper half, nerves $3-5$ per side. Stipules $1.5-3 \mathrm{~mm}$ long $\ldots \ldots \ldots \ldots \ldots \ldots \ldots \ldots \ldots \ldots \ldots \ldots$ 20. M. korthalsii

b. Leaf blades: first pair of nerves ending in the lower half, nerves 3-10 per side. Stipules $1.6-10 \mathrm{~mm}$ long . . . . . . . . . . . . . . . 20

20a. Leaf blades: opposite pair different in shape, smaller of a pair obcordate . . . . . 
21a. Leaf blades drying brownish, upper surface usually with scattered glandular hairs. Petioles 2.5-24 mm long. Staminate flowers: 3-3.6 mm diameter. Fruits capsules,

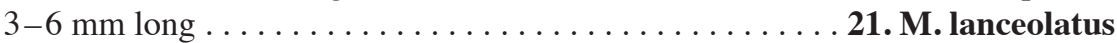

b. Leaf blades drying greenish, upper surface without scattered glandular hairs. Petioles 13-145 mm long. Staminate flowers: 4-7 mm diameter. Fruits drupes

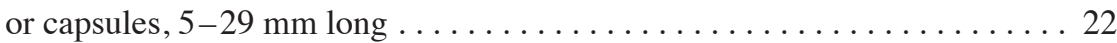

22a. Leaf blades: lower surface always with sparse glandular hairs, $4.5-8 \mathrm{~cm}$ wide. Petioles 30-62 mm. Fruits capsules ........... 35. M. subcuneatus

b. Leaf blades: lower surface with scattered or without glandular hairs, $1.5-16.5 \mathrm{~cm}$ wide. Petioles $13-145 \mathrm{~mm}$. Fruits drupes or capsules. . . . . . . . . 23

23a. Shrubs up to $3 \mathrm{~m}$ high. Leaves broadly ovate to elliptic. Staminate flowers: pedicels 2.5-3.5 mm long, stamens 19-35, filaments sparsely hairy. Pistillate flowers: sepals free, persistent. Fruits spiny capsules. - Thailand, Vietnam . . . . . . . . .

31. M. pierrei

b. (Large shrubs to) trees up to $30 \mathrm{~m}$ high. Leaves ovate. Staminate flowers: pedicels 3.9-10 mm long, stamens (25-)45-75(-130), filaments glabrous. Pistillate flowers: calyx connate, early caducous. Fruits rugose drupes. - India, Nepal, throughout mainland SE Asia to southern China, W Malesia . . . . . . . . . . .

D. M. nudiflorus (see Kulju et al., 2007)

\section{Key to the species of the Philippines, Sulawesi, Lesser Sunda Islands and the Moluccas}

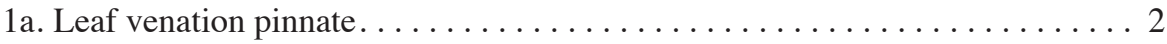

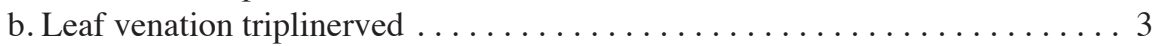

2a. Glandular hairs in most parts densely present. Staminate flowers $2.5-4 \mathrm{~mm}$ diameter. Pistillate flowers: calyx basally connate, persistent. Fruits spiny capsules. - S India, Sri Lanka, Andaman \& Nicobar Islands, Thailand, Laos, Vietnam, Philippines, Borneo, Java, Lesser Sunda Islands, Sulawesi, Moluccas, Papua New Guinea, Australia . . . . . . . . . . . . . . . . . 32. M. resinosus

b. Glandular hairs in most parts absent (to scattered present). Staminate flowers 3.2-5.2 (-6.9) $\mathrm{mm}$ diameter. Pistillate flowers: calyx entirely connate, early caducous. Fruits smooth to slightly verrucose drupes. - Borneo, Philippines, Sulawesi . . .

B. M. cumingii (see Kulju et al., 2007)

3a. Lower surface of leaf blades without or with scattered glandular hairs. Pistillate flowers: sepals connate, opening further with 1 or 2 sutures, early caducous. Fruits rugose drupes . . . . . . . . . . . . D. M. nudiflorus (see Kulju et al., 2007)

b. Lower surface of leaf blades with dense to sparse glandular hairs. Pistillate flowers: sepals free, persistent. Fruits spiny capsules . . . . . . . . . 4

4a. Stipules absent. Lower surface of leaf blades densely hairy, domatia absent ....

37. M. tiliifolius

b. Stipules present. Lower surface of leaf blades sparsely hairy to subglabrous,

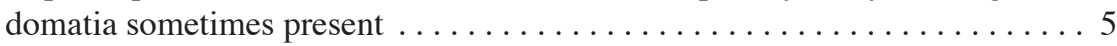

5a. Upper surface of leaf blades with glandular hairs. Stipules $18-30$ by $8-10 \mathrm{~mm}$. - Philippines. 
b. Upper surface of leaf blades without glandular hairs. Stipules $1.5-10$ by $0.5-4$

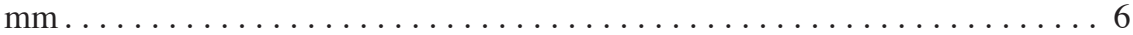

6a. Leaf blades: opposite pair different in shape, smaller of a pair obcordate . . . . .

26. M. minimifructus

b. Leaf blades: opposite pair similar in shape $\ldots \ldots \ldots \ldots \ldots \ldots \ldots \ldots$

7a. Leaf blades: first pair of nerves ending in upper half, extrafloral nectaries marginal from base (to apex); domatia sometimes present . . . . . 20. M. korthalsii

b. Leaf blades: first pair of nerves ending in lower half, extrafloral nectaries marginal from base to apex, sometimes also all over the blade, domatia always present . 8

8a. Leaf blades: ovate to obovate, apex acute to acuminate, nerves 8 or 9 per side. Stipules 2-4 mm wide, persistent to caducous . . . . . . 23. M. leucocalyx

b. Leaf blades: ovate to elliptic, apex caudate, nerves 4-6(-9) per side. Stipules 0.5-1

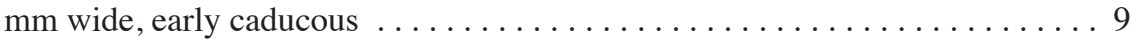

9a. Leaf blades drying green to green-brown. Stipules linear-triangular. Pistillate inflorescences with entire or trilobed bracts; pistillate flowers: buds ellipsoid, style 2-4 mm long. Staminate flowers with connate filaments. - Borneo, Philippines

8. M. connatus

b. Leaf blades drying brown. Stipules narrowly-triangular. Pistillate inflorescences with trilobed bracts; pistillate flowers: buds ovoid, style 0-1(-2) mm long. Staminate flowers with free to slightly connate filaments. - Sumatra, Java, Borneo, Lesser Sunda Islands . . . . . . . . . . . . . . . . . 33. M. rufidulus

\section{Key to the species of New Guinea,Australia,Palau,Micronesia, Solomon Islands and Fiji}

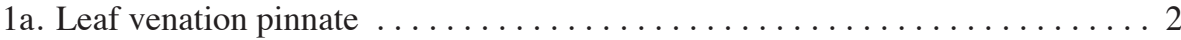

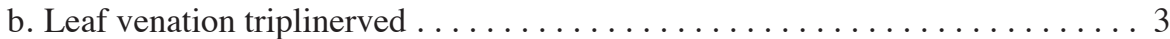

2a. Glandular hairs absent to rarely present. Stipules linear-triangular, $0.2-0.3 \mathrm{~mm}$ wide. Leaf blades narrowly elliptic, membranaceous. - New Guinea . . . . . . . .

2. M. attenuatus

b. Glandular hairs present. Stipules triangular to narrowly triangular, 1-1.7 mm wide. Leaf blades elliptic to obovate, chartaceous. - S India, Sri Lanka, Andaman \& Nicobar Islands, Thailand, Laos, Vietnam, Philippines, Borneo, Java, Lesser Sunda Islands, Sulawesi, Moluccas, Papua New Guinea, Australia . . 32. M. resinosus

3a. Glandular hairs absent . . . . . . . . . . . . . . . . . . . . . 4

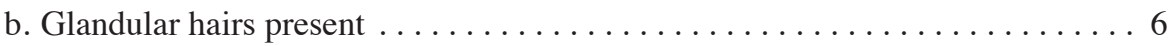

4a. Stipules 1-1.8 mm long. Leaf blades length/width ratio 1.1-1.4, domatia absent. Staminate inflorescences with 35-47 nodes per branch. Pistillate inflorescences racemes $\ldots \ldots \ldots \ldots \ldots \ldots \ldots \ldots \ldots \ldots \ldots \ldots \ldots \ldots$ 12. M. didymochryseus

b. Stipules 3-7 mm long. Leaf blades length/width ratio 1.6-2.6, domatia present. Staminate inflorescences with 5-8 nodes per branch. Pistillate inflorescences

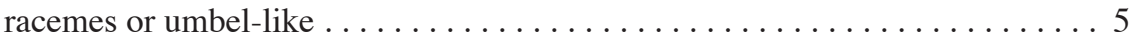

5a. Leaf blades: lower surface with scattered hairs, apex cuspidate. Staminate inflorescences 5-14 cm long; flowers: pedicels 6-10 $\mathrm{mm}$ long, filaments hairy. Pistillate inflorescences $2.7-4 \mathrm{~cm}$ long . . . . . . . . . . . 22. Muterbachianus 
b. Leaf blades: lower surface with sparse hairs, apex acute, acuminate to caudate. Staminate inflorescences 4.5-6 cm long; flowers: pedicels 3-4.5 mm long, filaments glabrous. Pistillate inflorescences $12-17 \mathrm{~cm}$ long . . 25. M. macularis

6a. Extrafloral nectaries along the midrib, also all over the blade, domatia absent. Fruits spiny, spines $500-600 \ldots \ldots \ldots \ldots \ldots \ldots \ldots \ldots \ldots \ldots$. . . darbyshirei

b. Extrafloral nectaries marginal, domatia sometimes present. Fruits smooth or spiny,

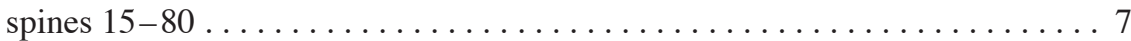

7a. Petioles $0.8-1 \mathrm{~mm}$ wide. Leaf blades: lower surface sparsely hairy, glabrescent, domatia sometimes present. Stamens 29-45. Fruits spiny . . 7. M. claoxyloides

b. Petioles 1-2.5 mm wide. Leaf blades: lower surface densely to sparsely hairy, domatia absent. Stamens $45-120$. Fruits smooth or spiny $\ldots \ldots \ldots \ldots \ldots 8$

8a. Leaf blades broadly ovate to ovate, length/width ratio 1-1.3. Stipules absent. Staminate flowers $2.8-5.5 \mathrm{~mm}$ diam.; filaments $0.5-2.3 \mathrm{~mm}$ long. Fruits 5-15 mm long, surface spiny. - Thailand to Taiwan, throughout Peninsular Malaysia, Malesia, Republic of Palau, and Federated States of Micronesia to the W Pacific

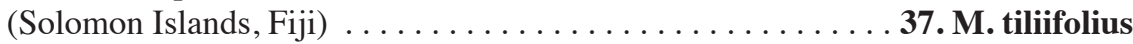

b. Leaf blades ovate to elliptic, length/width ratio 1.4-1.8. Stipules present. Staminate flowers 5-6 mm diam.; filaments $2.5-5 \mathrm{~mm}$ long. Fruits $17-22.5 \mathrm{~mm}$ long, surface smooth. - New Guinea . . . . . . . . . . . . . . . . 38. M. trinervius

\section{SYNOPTICAL KEY}

Bold $=$ feature constant for the species; roman $=$ feature variable; number absent $=$ feature absent from respective species. Numbers refer to the species number used in this revision. A-G refers to species treated in other publications: A, C, F, G in Van Welzen \& Sierra, 2006 and B, D, E in Kulju et al., 2007.

\begin{tabular}{|c|c|}
\hline & $=$ M. actinoneurus \\
\hline & $=$ M. attenuatus \\
\hline & $=$ M. blumeanus \\
\hline & $=M \cdot$ brevipetiolatus \\
\hline 5 & $=$ M. calocarpus \\
\hline 6 & $=$ M. cauliflorus \\
\hline 7 & $=$ M. claoxyloides \\
\hline 8 & $=M \cdot$ connatus \\
\hline 9 & $=M \cdot$ coudercii \\
\hline 10 & $=M$. darbyshirei \\
\hline 11 & $=M \cdot$ decipiens \\
\hline 12 & $=$ M. didymochryseus \\
\hline 13 & $=$ M. dispar \\
\hline 14 & $=$ M. eximius \\
\hline 15 & $=$ M. glabriusculus \\
\hline 16 & $=$ M. glomerulatus \\
\hline 17 & $=$ M. hispidospinosus \\
\hline 18 & $=M$. hymenophyllus \\
\hline 19 & $=$ M. khasianus \\
\hline 20 & $=M$. korthalsii \\
\hline 21 & $=$ M. lanceolatus \\
\hline 22 & $=$ M. lauterbachianus \\
\hline 0 & $=$ M. leucocaly \\
\hline
\end{tabular}

\begin{tabular}{|c|c|}
\hline & ginervis \\
\hline & $=$ M. macularis \\
\hline & $=\quad M \cdot$ minimifructu \\
\hline & $=$ M. mirus \\
\hline & $=M \cdot$ monanthos \\
\hline & $=M \cdot$ montanus \\
\hline & $=$ M. oppositifoliu. \\
\hline & $=$ M.pierrei \\
\hline & $=M \cdot$ resinosus \\
\hline & $=$ M.rufidulus \\
\hline & $=$ M. sphaerocarp \\
\hline & $=M \cdot$ subcuneatus \\
\hline & $=$ M. subulatus \\
\hline & $=M \cdot$ tiliifolius \\
\hline & $=M$. trinervius \\
\hline & $=$ M. caudatus \\
\hline & $=M \cdot$ cumingii \\
\hline & $=M \cdot$ lancifolius \\
\hline & $=M \cdot$ nudiflorus \\
\hline & $=M \cdot$ polycarpus \\
\hline & $=M \cdot$ spinifruct \\
\hline & $=M \cdot$ wrayi \\
\hline
\end{tabular}




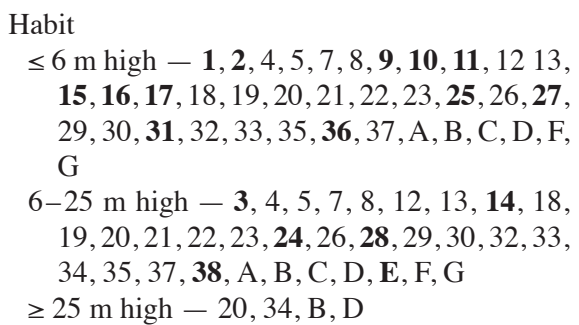

Non-glandular indument type

Simple hairs - 1,2,4, 5, 6, 7, 8, 9, 10, 11, 12, $13,14,15,16,17,18,19,20,21,22,23,24$, $25,26,27,28,29,30,31,32,33,35,36,37$, 38, A, B, C, D, E, F, G

(Stellately-)tufted hairs - 1, 2, 3, 4, 5, 6, 7, 8, $9,10,11,12,13,14,15,16,17,18,19,20$, $21,22,23,24,25,26,27,28,29,30,31,32$, 33, 34, 35, 36, 37, 38, A, B, D, E, F, G

Glandular hairs

Absent - 1, 2, 5, 12, 16, 22, 25, 27, 28, B, D, E

Present $-2, \mathbf{3}, \mathbf{4}, 5,6,7, \mathbf{8}, \mathbf{9}, \mathbf{1 0}, \mathbf{1 1}, \mathbf{1 3}, \mathbf{1 4}$, $15,17,18,19,20,21,23,24,26,29,30,31$,

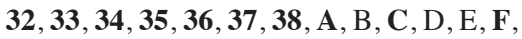
G

\section{Stipule shapes}

\section{Deltoid - 24, 29}

Triangular - 4, 5, 7, 9, 10, 11, 12, 16, 18, 20, $21,23,26,27,29,30,32,38, \mathbf{A}, \mathbf{B}, \mathbf{C}, \mathbf{D}$, F, G

Narrowly triangular $-\mathbf{1}, \mathbf{3}, 5, \mathbf{6}, 7,9,12, \mathbf{1 3}$, 14, 18, 19, 21, 22, 23, 25, 26, 27, 30, 31, 32, 33, 34, 38, B, D

Linear-triangular - 2, 7, 8, 9, 15, 17, 19, 28, 31, 35, 36,

Obovate - B

Stipule lengths ( $\mathrm{mm})$

$<5-2,3,4,7,8,11,12,15,16,17, \mathbf{1 8}, \mathbf{1 9}$, 20, 21, 22, 23, 24, 25, 26, 29, 30, 31, 32, 33, $34, \mathbf{3 5}, 36, \mathbf{3 8}, \mathrm{A}, \mathrm{B}, \mathrm{C}, \mathrm{D}$,

5.01-10-1, 5, 7, 8, 9, 10, 13, 14, 15, 17, 23, $25,26,27,28,31,33,34,36, A, B, C, D, \mathbf{F}$, G

$10.01-15-1,9,14,28,36$

$\geq 15-6$

Stipule persistence

Persistent - 1, 6, 7, 9, 13, 15, 21, 23, 26, 27, 31,36

(Early) Caducous - 2, 3, 4, 5, 7, 8, 9, 10, 11, 12, $13, \mathbf{1 4}, 15, \mathbf{1 6}, \mathbf{1 7}, \mathbf{1 8}, \mathbf{1 9}, \mathbf{2 0}, 21, \mathbf{2 2}, 23$,
24, 25, 26, 28, 29, 30, 31, 32, 33, 34, 35, 36, 38, A, B, C, D, E, F, G

Absent $\mathbf{-} \mathbf{3 7}$

Blade shapes

Ovate $-3,8,9, \mathbf{1 2}, 13,14,15,17, \mathbf{1 8}, 20,21$, $22,23,25,26,29,30,31,33,34,35,36,37$, $38, \mathrm{~A}, \mathrm{~B}, \mathrm{C}, \mathbf{D}, \mathbf{E}, \mathrm{F}, \mathrm{G}$

Elliptic - 2, 3, 4, 5, 6, 7, 8, 9, 10, 13, 14, 15, 16, 17, 19, 20, 21, 22, 23, 24, 25, 26, 29, 31, $32,33,34,35,36,38, A, B, C, F, G$

Obovate - 1, 4, 5, 6, 7, 9, 10, 11, 13, 15, 19, $20,21,23,26,27,28,32, \mathrm{~B}, \mathrm{C}$

Blade length/width ratios

$1-2-1,5,6,7,8,9,10,11,12,13,14,15$, 18, 19, 20, 21, 22, 23, 24, 25, 26, 27, 29, 30, $31,32,33, \mathbf{3 4}, 35,36, \mathbf{3 7}, \mathbf{3 8}, \mathrm{B}, \mathrm{C}, \mathrm{D}, \mathbf{E}$

2.01-3 - 1, 2, 3, 4, 5, 6, 7, 8, 10, 11, 13, 14, $15,16,17,19,21,22,24,25,26,27,29,30$,

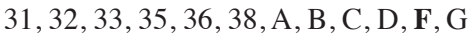

$>3.01-1,2,4,15,16,17,19,26,28,30,32$, A, B, C, G

Blade bases

Truncate - 30, 31, 36, 37, E

Cordate - 12, 14, 15, 18, 29, 30, 36, 37, D, E, F

Rounded - 3,6,7,8,9,12,13,14, 15, 20,22, $23,25,30,31,33,35,37,38, \mathrm{C}$

Obtuse - 3, 6, 7, 8, 9, 10, 11, 13, 14, 15, 19, $20,22,24,25,26,29,30,31,32,33,34,35$, $36,38, \mathrm{~B}, \mathrm{D}$

Cuneate - 2, 3, 4, 6, 7, 8, 10, 11, 13, 19, 20, 21, 22, 23, 24, 25, 26, 27, 31, 32, 33, 34, 35, 37, A, B, C, D, F, G

(Shallowly) Attenuate - 2, 5, 16, 28, 32, 38, B, C

(Shallowly) Emarginate - 1, 4, 7, 9, 17, 19, $23,25,26, \mathrm{C}$

Blade apices

Acute - 7, 9, 12, 15, 18, 21, 23, 25, 29, 31, $32,35,36,37,38, \mathrm{C}, \mathrm{D}$

Acuminate - 3, 4, 6, 7, 12, 13, 15, 18, 21, 23, $25,29,30,31,32,36,37,38, \mathrm{~B}, \mathrm{C}, \mathrm{D}, \mathbf{E}$

Caudate - 1, 2, 3, 4, 5, 6, 8, 10, 11, 13, 14, $15,16, \mathbf{1 7}, \mathbf{1 9}, \mathbf{2 0}, \mathbf{2 4}, 25, \mathbf{2 6}, \mathbf{2 7}, \mathbf{2 8}, 29,30$, $32, \mathbf{3 3}, 34,36,37, \mathrm{~A}, \mathrm{C}, \mathbf{F}, \mathrm{G}$

Cuspidate - 3, 5, 9, 11, 16, 22, 32, 34, 35, A, B, G

Obtuse - 7, 38, B

Rounded $-7,9,12,15, \mathrm{~B}$ 
Extrafloral nectary positions (excluding the minute, orbicular, extrafloral nectaries which are sometimes present on the upper half of the leaf)

Basal (one pair near the petiole insertion always present and bigger than the other extrafloral nectaries) $-\mathbf{3}, \mathbf{3 4}, \mathrm{A}, \mathrm{B}, \mathbf{C}, \mathrm{D}, \mathbf{F}, \mathbf{G}$

Marginal (two to several pairs along the entire margin or only on the lower half, if near the petiole insertion (e.g., when the leaf base is attenuate), usually the same size as the other extrafloral nectaries) $-\mathbf{1}, \mathbf{2}, 4, \mathbf{5}, 7,8, \mathbf{9}, \mathbf{1 1}$, $\mathbf{1 2}, \mathbf{1 3}, 14,15, \mathbf{1 6}, \mathbf{1 7}, \mathbf{1 8}, \mathbf{1 9}, \mathbf{2 0}, \mathbf{2 1}, 23, \mathbf{2 4}$, $26,27,28,29,30,31,32,33,35,36,37,38$, $\mathrm{B}, \mathrm{D}, \mathbf{E}$

Along midrib throughout the leaf blade $\mathbf{- 1 0}$, 22, 25

Touching midrib $-4,32$

On axils (between midrib and nerves) $-\mathbf{6}$, 15

All over the blade $-8,14,23,25,33$

\section{Venation type}

Pinnate - 1, 2, 4, 5, 16, 17, 19, 27, 28, 32, B

Triplinerved $-\mathbf{3}, \mathbf{6}, \mathbf{7}, \mathbf{8}, \mathbf{9}, \mathbf{1 0}, \mathbf{1 1}, \mathbf{1 2}, \mathbf{1 3}, \mathbf{1 4}$, $15,18,20,21,22,23,24,25,26,29,30,31$, $33,34,35,36,37,38, A, C, D, E, F, G$

Number of nerves per side

$\leq 5-8,9,10,15,18, \mathbf{2 0}, 21,22, \mathbf{2 4}, 29,30$, $31,33,35,36,38, \mathrm{D}$

$6-10-\mathbf{1}, 2, \mathbf{3}, 4, \mathbf{5}, 6,7,8,9,10, \mathbf{1 1}, \mathbf{1 2}, \mathbf{1 3}$, $\mathbf{1 4}, 15, \mathbf{1 6}, \mathbf{1 7}, 18,19,21,22, \mathbf{2 3}, \mathbf{2 5}, \mathbf{2 6}, \mathbf{2 7}$, $29,30,31,32,33, \mathbf{3 4}, 35,36,37,38, \mathbf{A}, \mathrm{B}$, $\mathbf{C}, \mathrm{D}, \mathbf{E}, \mathbf{F}, \mathbf{G}$

$>10-2,4,6,19,28,32,37, \mathrm{~B}$

\section{Domatia}

Present - 2, 6, 7, 9, 11, 13, 15, 18, 19, 20, 21, $\mathbf{2 2}, 24, \mathbf{2 5}, 26,29, \mathbf{3 0}, 36, \mathbf{A}, \mathrm{B}, \mathbf{C}, \mathrm{D}, \mathbf{F}, \mathbf{G}$ Absent - 1, 2, 3, 4, 5, 6, 7, 8,9, 10,11, 12, 13, $\mathbf{1 4}, \mathbf{1 6}, \mathbf{1 7}, 19,20,21, \mathbf{2 3}, 24,26, \mathbf{2 7}, \mathbf{2 8}, 29$, $\mathbf{3 1}, \mathbf{3 2}, \mathbf{3 3}, \mathbf{3 4}, \mathbf{3 5}, 36, \mathbf{3 7}, \mathbf{3 8}, \mathrm{B}, \mathrm{D}, \mathbf{E}$

Staminate inflorescence lengths $(\mathrm{cm})$

$$
\begin{aligned}
& \leq 5-\mathbf{2}, \mathbf{5}, \mathbf{7}, \mathbf{9}, \mathbf{1 5}, \mathbf{1 6}, \mathbf{1 7}, 20,21,22,23,25, \\
& \mathbf{2 9}, 30,32, \mathbf{3 5}, 36, \mathrm{~F}, \mathrm{G} \\
& 5.01-19.99-\mathbf{3}, \mathbf{4}, \mathbf{6}, 8, \mathbf{1 1}, \mathbf{1 3}, \mathbf{1 4}, 19,20,21, \\
& 22,23, \mathbf{2 4}, 25, \mathbf{2 6}, 30, \mathbf{3 1}, 32,33,34,36,37, \\
& \mathbf{3 8}, \mathbf{A}, \mathrm{B}, \mathrm{C}, \mathrm{D}, \mathrm{F}, \mathrm{G} \\
& \geq 20-3,8, \mathbf{1 2}, 19,20,30,33,34,37, \mathrm{~B}, \mathrm{C},
\end{aligned}
$$$$
\text { D }
$$

Staminate flower diameters ( $\mathrm{mm}$ )

$$
\begin{aligned}
\leq & 3.5-2, \mathbf{3}, 4, \mathbf{6}, 8,11,13,16, \mathbf{1 7}, 18,20,21, \\
& 23, \mathbf{2 4}, 26, \mathbf{2 9}, 30,32,33, \mathbf{3 4}, 37, \mathrm{~A}, \mathrm{~B}, \mathrm{C}, \\
& \mathrm{G}
\end{aligned}
$$

$3.51-4.99-2,4,8,9,11,13,14,15,16,18$, $20,21,23,26,28,30,31,32,33,35,36,37$, A, B, C, D, G

$\geq 5-\mathbf{5}, \mathbf{7}, 9, \mathbf{1 2}, 14,15, \mathbf{1 9}, 20, \mathbf{2 2}, \mathbf{2 5}, 28$, $30,31,35,36,37,38, \mathrm{~B}, \mathrm{C}, \mathrm{D}$

Disc-glands

Absent $-2,3,4,5,6,7,8,9,11,12,13,14$, $15,16,17,18,20,21,22,23,24,25,28,29$, 30, 31, 32, 33, 34, 35, 36, 37, 38, A, C, D

Present $-19,26, \mathbf{B}, \mathbf{G}$

Stamen numbers

$<50-2,3,4,5,6,7,8,9,11,13,14,15,16$, $18,19, \mathbf{2 0}, 21,22, \mathbf{2 3}, \mathbf{2 4}, 26, \mathbf{2 8}, \mathbf{2 9}, 30, \mathbf{3 1}$, $\mathbf{3 2}, \mathbf{3 4}, \mathbf{3 5}, 36,37, \mathrm{~A}, \mathrm{~B}, \mathrm{C}, \mathrm{D}, \mathbf{G}$

$50-99-5,8, \mathbf{1 2}, 13,15, \mathbf{1 7}, 18,19,21,22$, $\mathbf{2 5}, 26,30, \mathbf{3 3}, 36,37, \mathrm{~A}, \mathrm{~B}, \mathrm{C}, \mathrm{D}$

$\geq 100-\mathbf{3 8}$

Filament lengths $(\mathrm{mm})$ $<1.99-2, \mathbf{3}, 4,6,7,11,12,13, \mathbf{1 6}, \mathbf{1 7}, \mathbf{1 8}$, $19,21,22,23, \mathbf{2 4}, 26,30,31,32,33, \mathbf{3 4}, 37$, A, B, C, D, G

$2-3-2,4, \mathbf{5}, 6,7, \mathbf{9}, 11,12,13,14, \mathbf{1 5}, 19$, $\mathbf{2 0}, 21,22,23,25,26,28,29,30,31,32,33$, $35,37,38, A, B, C, D, G$

$>3.01-7, \mathbf{8}, 14,19,22,25,28,33,35, \mathbf{3 6}$, $38, \mathrm{~B}, \mathrm{C}, \mathrm{D}$

\section{Stamen connectivity}

Free $-2,3,4,5,6,7,9,11,12,13,15,16$, $17,18,19,20,21,22,23,24,25,26,29,30$,

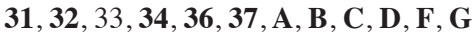

Connate $-\mathbf{8}, \mathbf{1 4}, \mathbf{2 8}, 33,35$

Filament indumentum

Hairy $-5,9,14,15,20,22,29,31,35,36, A$, $\mathbf{C}, \mathbf{G}$

Glabrous - 2, 3, 4, 5, 6, 7, 8, 11, 12, 13, 15, $16,17,18,19,20,21,23,24,25,26,28,30$, 32, 33, 34, 37, 38, B, D

Connective type

Umbrella-like - 3, 6, 34

Widened $-2,7,8,9,11,12,13,15,17,19$, $21,22,23,25,26,28,31,32,35,36,37,38$, A, B, C, D, G

Not widened $-4,5,14,16,18,20,24,29$, 30, 33, B, D, G

Pistillode

Present $-2,7,8,9,11,12,13,14,15,16,21$, $22,23,25,28,29,31,32,33,35,36,37$, 38

Absent $-3,4,5,6,17,18,19,20,24,26,30$, 34, A, B, C, D, G 
Pistillate inflorescence types

Racemes - 3, 4, 6, 8, 11, 12, 13, 14, 15, 17, $18,19,20,21,22,23,24,26,29,30,31,32$, 33, 34, 35, 36, 37, A, B, C, D, E, F, G Umbel-like - 7, 9, 10, 15, 22, 25, 36

Reduced to one flower $-\mathbf{1}, \mathbf{5}, \mathbf{1 6}, \mathbf{2 7}, \mathbf{2 8}$, D

Pistillate inflorescence lengths $(\mathrm{cm})$

$$
\begin{aligned}
& \leq 2-\mathbf{5}, \mathbf{7}, 15, \mathbf{1 6}, \mathbf{2 7}, \mathbf{2 8}, 36, \mathrm{D} \\
& 2.01-10-3, \mathbf{4}, 6,9, \mathbf{1 0}, 11,12,13,15, \mathbf{1 7}, \\
& \quad 18,20, \mathbf{2 1}, \mathbf{2 2}, 23,24,26, \mathbf{2 9}, 30,31, \mathbf{3 2}, 34, \\
& \mathbf{3 5}, 36,37, \mathrm{~B}, \mathrm{C}, \mathrm{D}, \mathbf{E}, \mathbf{F}, \mathrm{G} \\
& 10.01-20-3,6,8,9,11,12,13,14,18,20, \\
& \quad 23,24, \mathbf{2 5}, 26,30,31,33,34,37, \mathrm{~A}, \mathrm{~B}, \mathrm{C}, \\
& \mathrm{D}, \mathrm{G} \\
& > \\
& 20-8,14, \mathbf{1 9}, 20,33, \mathrm{~A}
\end{aligned}
$$

Pistillate flower diameters $(\mathrm{mm})$

$$
\begin{aligned}
& \leq 2.5-\mathbf{1}, 4, \mathbf{1 1}, 13,15,21, \mathbf{2 4}, \mathbf{3 2}, 35,37, \mathbf{B}, \\
& \text { C, E, G } \\
& 2.51-3.99-3,4,6, \mathbf{9}, 12,13,15,18, \mathbf{2 0}, 21, \\
& \quad 22, \mathbf{2 3}, \mathbf{2 5}, \mathbf{2 6}, \mathbf{3 0}, \mathbf{3 1}, 35,36,37, \mathrm{C}, \mathrm{G} \\
& 4-6-3, \mathbf{5}, 6, \mathbf{7}, \mathbf{8}, \mathbf{1 0}, 12,14,15, \mathbf{1 6}, \mathbf{1 7}, 18, \\
& \quad 19,22, \mathbf{2 9}, \mathbf{3 3}, \mathbf{3 4}, 36,37, \mathbf{D} \\
& >6-7,14,19, \mathbf{A}, \mathbf{F}
\end{aligned}
$$

Ovary locularity

$$
\begin{aligned}
& 1-\mathrm{B} \\
& 2-9, \mathbf{1 2}, 15,31,38, \mathrm{~B}, \mathrm{E}, \mathrm{G} \\
& 3-\mathbf{1}, \mathbf{3}, \mathbf{4}, \mathbf{5}, \mathbf{6}, \mathbf{7}, \mathbf{8}, 9, \mathbf{1 0}, \mathbf{1 1}, \mathbf{1 3}, \mathbf{1 4}, 15, \mathbf{1 6}, \\
& \mathbf{1 7}, \mathbf{1 8}, \mathbf{1 9}, \mathbf{2 0}, \mathbf{2 1}, \mathbf{2 2}, \mathbf{2 3}, \mathbf{2 4}, \mathbf{2 5}, \mathbf{2 6}, \mathbf{2 7}, \mathbf{2 8}, \\
& \mathbf{2 9}, 30,31, \mathbf{3 2}, \mathbf{3 3}, \mathbf{3 4}, \mathbf{3 5}, 36,37,38, \mathbf{A}, \mathrm{C}, \\
& \quad \mathrm{D}, \mathrm{F}, \mathrm{G} \\
& 4-9,30,31,36,37,38, \mathrm{D}, \mathrm{F} \\
& 5-\mathrm{D}
\end{aligned}
$$

\section{Fruit types \\ Capsules - 1, 4, 5, 6, 7, 8, 9, 10, 11, 12, 13, $14,15,16,17,18,19,20,21,23,24,25,26$, $27,28,29,30,31,32,33,35,36,37,38, A$, $\mathbf{C}, \mathbf{E}, \mathbf{F}, \mathbf{G}$}

Indehiscent drupes $-\mathbf{3}, \mathbf{3 4}, \mathbf{B}, \mathbf{D}$

Fruit lengths (mm)

$<10-\mathbf{4}, 6, \mathbf{7}, \mathbf{9}, \mathbf{1 0}, \mathbf{1 1}, 13,15,16, \mathbf{1 7}, 18$, $19,20, \mathbf{2 1}, \mathbf{2 3}, \mathbf{2 4}, \mathbf{2 5}, 26,29, \mathbf{3 0}, \mathbf{3 1}, \mathbf{3 2}, 33$, $35,37, \mathrm{~A}, \mathrm{~B}, \mathrm{C}, \mathrm{G}$

$10-15-1, \mathbf{3}, 5,6, \mathbf{8}, \mathbf{1 2}, 13,15,16,18,19$, $20,26, \mathbf{2 7}, 29,33,34,35, \mathbf{3 6}, 37, \mathrm{~A}, \mathrm{~B}, \mathbf{E}$, F, G

$>15-1,5, \mathbf{1 4}, 19, \mathbf{2 8}, 33,34, \mathbf{3 8}, \mathrm{D}, \mathrm{G}$

Fruit widths (mm)

$<10-\mathbf{3}, 4,6,9, \mathbf{1 1}, 13,18, \mathbf{2 1}, 23, \mathbf{2 4}, 26$, 30, 31, 32, 33, 36, 37, B, C, G
$10-15-4,6,7,8,9, \mathbf{1 0}, 12,13,15, \mathbf{1 6}, 17$, $18,19, \mathbf{2 0}, 23,25,26,27,29,31,32,33,34$, $35,36,37, \mathrm{~A}, \mathrm{~B}, \mathrm{C}, \mathbf{E}, \mathrm{F}, \mathrm{G}$

$>15-\mathbf{1}, \mathbf{5}, 8,12, \mathbf{1 4}, 15,17,19,25, \mathbf{2 8}, 29$, $33,34,35, \mathbf{3 8}, \mathrm{A}, \mathrm{D}, \mathrm{F}, \mathrm{G}$

Fruit dehiscence types

Septicidally-loculicidally $-\mathbf{1 , 4}, \mathbf{5}, \mathbf{6}, \mathbf{7}, \mathbf{8}, \mathbf{9}$, $10,11,13,14,15,16,17,18,19,20,21,23$, $24,25,26,27,28,29,30,31,32,33,35,36$, 37, A, C, F, G

Loculicidally $-\mathbf{1 2}, \mathbf{3 8}, \mathrm{E}$

Fruit smoothness

Not spiny (densely hairy to glabrous, rarely wrinkled) $-1,3,5,9,12,16,24,27,28$, 29, 30, 38, B, D, E

Spiny $-4,6,7,8,10,11,13,14,15,17,18$, $19,20,21,23,25,26,31,32,33,35,36,37$, $\mathbf{A}, \mathbf{C}, \mathbf{F}, \mathbf{G}$

Verrucose $-30, \mathbf{3 4}, \mathrm{B}$

Rugose - D, E

Spine numbers

$<100-\mathbf{4}, 6,7, \mathbf{1 3}, 18,19,21, \mathbf{2 5}, 31, \mathbf{3 2}, \mathbf{3 7}$, $\mathrm{C}$

$100-400-6, \mathbf{8}, \mathbf{1 1}, \mathbf{1 5}, 18,19, \mathbf{2 0}, 21, \mathbf{2 3}$, $\mathbf{2 6}, 31,33,35,36, A, C, F, G$

$>400-10,14,17$

Wall thickness (mm) $\leq 0.5-3,9,11,12,15,21,24,29,30,34,35$, A, B, C, E

$0.51-1.5-4,5,6,7,8,10,13,14,16,17,18$, $19,20,21,23,25,26,27,28,31,32,33,36$. 37, A, B, C, E, F, G $>1.5-\mathbf{1}, 14, \mathbf{3 8}, \mathrm{B}, \mathrm{D}$

Seed shapes

Globose or \pm globose $-\mathbf{1}, \mathbf{4}, \mathbf{5}, \mathbf{6}, \mathbf{7}, \mathbf{8}, \mathbf{9}, \mathbf{1 0}$, $11,12,13,14,15,16,17,18,19,20,21,23$, $24,25,26,27,28,29,30,31,32,33,35,36$, $37,38, \mathbf{A}, \mathbf{B}, \mathbf{C}, \mathbf{F}, \mathbf{G}$

Lenticular $-\mathbf{3}, \mathbf{3 4}$

Triangular to almost elliptic in cross section - D

Broadly ellipsoid but flattened against the septum $-\mathbf{E}$

Distribution

Africa - 30, 36

Madagascar - 30

India, Nepal, Bhutan - 19, 32, D, E

Sri Lanka - 32, D

Bangladesh - 11, D 


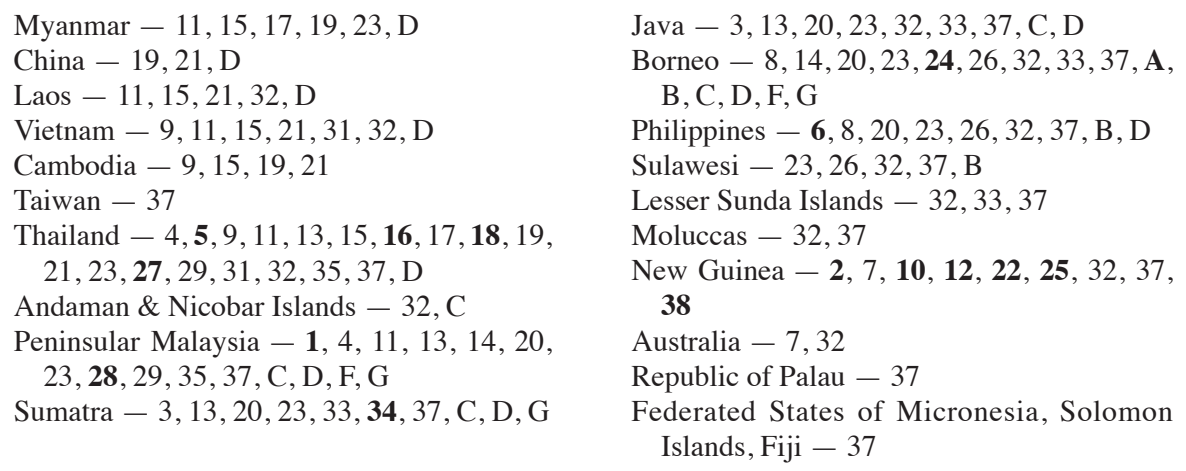

\section{Mallotus actinoneurus Airy Shaw - Map 1}

Mallotus actinoneurus Airy Shaw (1978b) 63. - Type: KEP FRI (Sohadi) 17911 (holo K; iso L), Peninsular Malaysia, Trengganu, Jerteh, Gunong Tebu Forest Reserve.

Shrubs, 1.5-3 m high, dioecious. Indumentum composed of simple, tufted and stellatelytufted hairs, glandular hairs absent. Stipules narrowly triangular, $10-13$ by $2.5-3.5$ $\mathrm{mm}$, persistent, margin entire, apex acute, densely to sparsely hairy. Leaves opposite to rarely alternate; petiole $14-61$ by $1.5-3 \mathrm{~mm}$, densely hairy; blade narrowly obovate, $25-32$ by $7-14 \mathrm{~cm}$, length/width ratio $2.1-3.6$, chartaceous, base shallowly emarginate, margin entire to denticulate, with glandular teeth, apex caudate, upper surface sparsely to scatteredly hairy on midrib and nerves, larger extrafloral nectaries marginal in lower half, 3 or 4 per side, 1-4 mm from margin, elliptic to orbicular, $0.7-0.8$ by

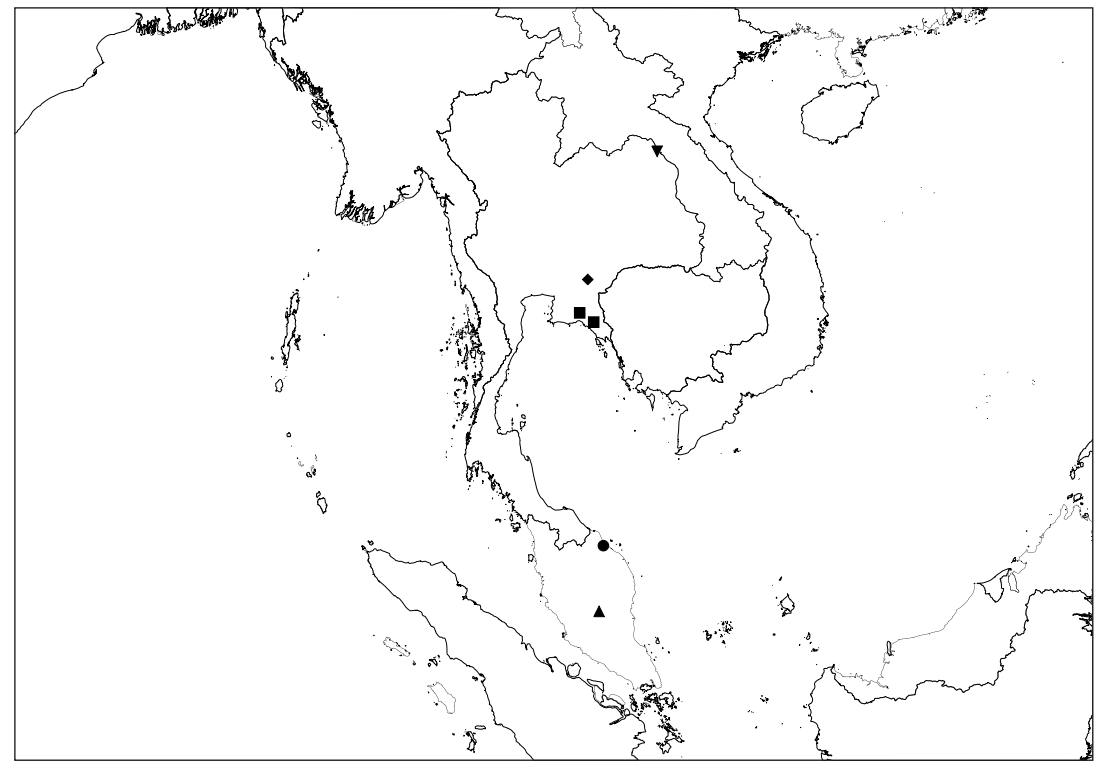

Map 1. Distribution of Mallotus actinoneurus Airy Shaw (•), M, calocarpus Airy Shaw (ם), M. glomerulatus Welzen $(\boldsymbol{\nabla})$, M. mirus S.E.C. Sierra $(\diamond)$, M. monanthos Airy Shaw $(\boldsymbol{\Delta})$. 
$0.7-0.8 \mathrm{~mm}$, smaller ones all over the blade, $0.2-0.3$ by $0.2-0.3 \mathrm{~mm}$, lower surface sparsely hairy, domatia absent, venation pinnate, nerves 9 or 10 per side, looping and closed near margin. Staminate inflorescences and flowers unknown. Infructescences racemes, with a single terminal fruit, c. $1.7 \mathrm{~cm}$ long, erect, single; peduncle c. $2 \mathrm{~mm}$ long; bracts linear-triangular, 3.5-7 by $0.7-1 \mathrm{~mm}$, margin entire or trilobed, apex acute, densely hairy; bracteoles absent. Pistillate flowers (based on young fruit) c. $10 \mathrm{~mm}$ diam.; pedicels c. $2.5 \mathrm{~mm}$ long, densely hairy; sepals 3, triangular to narrowly triangular, $8-10$ by c. $3 \mathrm{~mm}$, free, margin entire, apex acute; ovary c. 9 by $8 \mathrm{~mm}, 3$-locular, densely hairy; style c. $2.2 \mathrm{~mm}$ long; stigmas c. 6 by $1-1.2 \mathrm{~mm}$. Fruits capsules, $15-18$ by $23-27 \mathrm{~mm}$, opening septicidally-loculicidally, surface smooth, densely hairy; wall 1.8-2 mm thick, glabrous inside; column c. 11.5 by $11.5 \mathrm{~mm}$. Seeds \pm globose, c. 12 by 11.5 by $12 \mathrm{~mm}$, surface rugose, dull, light brown.

Distribution - Endemic to Peninsular Malaysia.

Habitat \& Ecology - In logged area. Altitude c. 80 m. Fruiting: April.

\section{Mallotus attenuatus Airy Shaw - Map 2}

Mallotus attenuatus Airy Shaw (1978b) 63. - Type: NGF (Streimann \& Kairo) 27781 (holo K; iso A, BO, BRI, CANB, SING), Papua New Guinea, Central Prov., Brown River.

Shrubs, dioecious. Indumentum composed of simple and stellately-tufted hairs, glandular hairs absent to rarely present. Stipules linear-triangular, $2-4$ by $0.2-0.3 \mathrm{~mm}$, caducous, margin entire, apex acute, scatteredly hairy to glabrous outside, glabrous inside. Leaves opposite; petiole $2-8$ by $0.8-1 \mathrm{~mm}$, densely to sparsely hairy; blade narrowly elliptic, $8.2-16$ by $1.7-4.7 \mathrm{~cm}$, length/width ratio $3.2-4.8$, membranaceous, base cuneate to

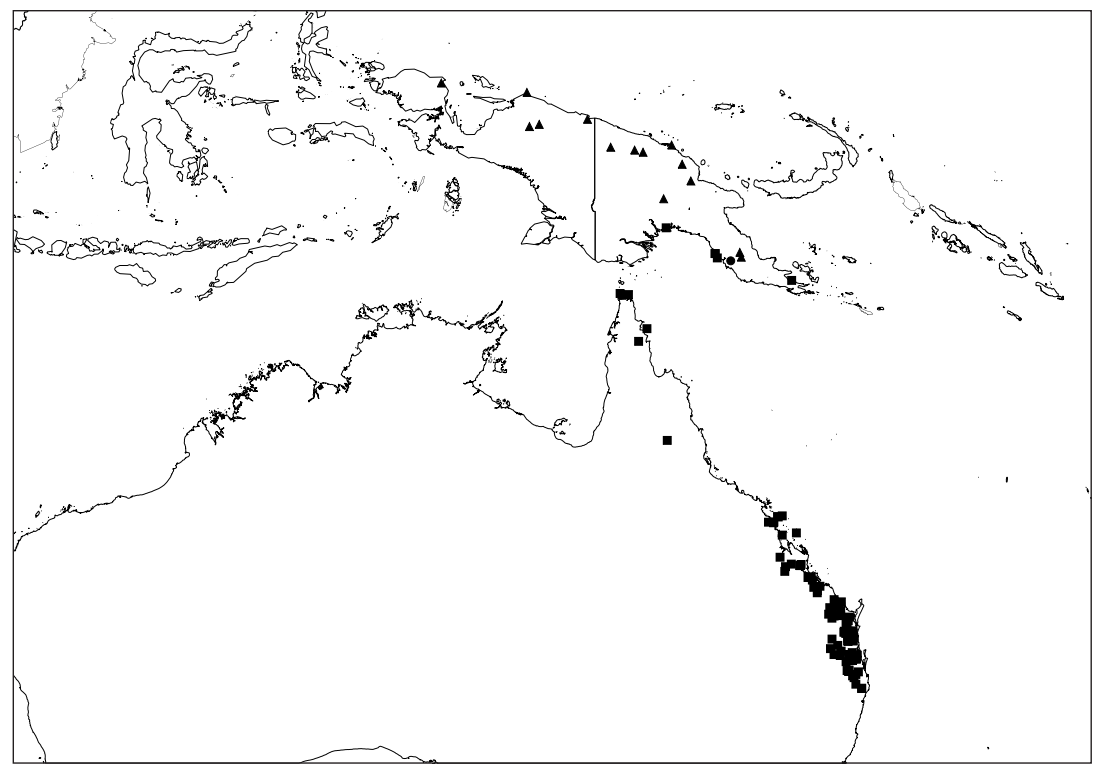

Map 2. Distribution of Mallotus attenuatus Airy Shaw (๑), M. claoxyloides (F. Muell.) Müll.Arg. (ם), M. trinervius (K. Schum. \& Lauterb.) Pax \& K. Hoffm. (A). 
attenuate, margin crenulate, without glandular teeth, apex caudate, upper surface glabrous, extrafloral nectaries sometimes present, inconspicuous, marginal in lower half, 1 per side, $0.2-0.5 \mathrm{~mm}$ from margin, elliptic to orbicular, $0.2-0.3$ by $0.2-0.3 \mathrm{~mm}$, lower surface scatteredly hairy, glabrescent, glandular hairs absent to rarely present (on midrib of young leaves), domatia sometimes present, venation pinnate, nerves 9-12 per side, looping and closed near margin. Staminate inflorescences reduced racemes, c. $0.5 \mathrm{~cm}$ long (still young, with buds), single, erect; peduncle $2-4 \mathrm{~mm}$ long; axes densely hairy, nodes per branch 2 or 3 , with 3-5 flowers per bract; bracts linear-triangular, $2-2.2$ by $0.2-0.3 \mathrm{~mm}$, persistent, apex acute, margin entire, scatteredly hairy to glabrous; bracteoles triangular, $0.5-1$ by $0.2-0.3$, persistent to caducous. Staminate flowers 3-4 mm diam.; pedicels $2.5-3 \mathrm{~mm}$ long, densely hairy; sepals 3 , elliptic to obovate, $2-2.2$ by $1-1.2 \mathrm{~mm}$, free, persistent, margin entire, apex acute, densely hairy outside, glabrous inside; stamens 25-30, glabrous, filaments $1.8-2.5 \mathrm{~mm}$ long, free, thecae ellipsoid, $0.2-0.3$ by $0.2-0.25 \mathrm{~mm}$, connective widened; pistillode present. Pistillate inflorescences, flowers and fruits unknown.

Distribution - New Guinea.

Habitat \& Ecology - Altitude c. 90 m. Flowering: February.

Note - Mallotus attenuatus differs from $M$. claoxyloides in the absence to rarely presence of glandular hairs (always present in $M$. claoxyloides), inconspicuous extrafloral nectaries, sometimes absent (conspicuous, always present in M. claoxyloides), 9-12 nerves per side (7 or 8 in $M$. claoxyloides) and a cuneate leaf base (shallowly emarginate, rounded, obtuse, to cuneate in M. claoxyloides).

\section{Mallotus blumeanus Müll.Arg. - Map 3}

Mallotus blumeanus Müll.Arg. (1865) 195; Backer \& Bakh.f. (1964) 483; Airy Shaw (1981) 325.

- Rottlera oppositifolia Blume (1826) 608, non Mallotus oppositifolius (Geiseler) Müll.Arg.

- Plagianthera oppositifolia (Blume) Rchb.f. \& Zoll. (1856) 20. - Rottlera blumeana (Müll.

Arg.) Scheff. (1869) 124. - Lectotype (selected here): Blume 1366, pro fem. (holo L, barcode L0291898; iso NY), Java, Salak, Parang.

Small trees, 13-23 m high; bole 4-7 m high, dbh 15-60 cm, dioecious, crown 7-14 m high. Outer bark smooth. Indumentum composed of stellately-tufted hairs and yellow to orange glandular hairs. Stipules narrowly triangular, $3.5-4$ by $0.8-1.2 \mathrm{~mm}$, early caducous, margin entire, apex acute, densely hairy. Leaves opposite; petiole 12-75 by $1-2.5 \mathrm{~mm}$, sparsely to scatteredly hairy; blade ovate to elliptic, sometimes slightly falcate, $13-33$ by $5-15 \mathrm{~cm}$, length/width ratio $2.1-2.8$, chartaceous, base rounded, obtuse to cuneate, margin denticulate to crenulate, sometimes with glandular teeth, apex acuminate to caudate, upper surface scatteredly hairy, larger extrafloral nectaries basal near the petiole insertion, $1-3$ per side, elliptic, $1.2-2$ by $0.5-1 \mathrm{~mm}$, smaller ones marginal in upper half, 5-14 nectaries per side, $0.8-2.8 \mathrm{~mm}$ from margin, orbicular, $0.2-0.3$ by $0.2-0.3 \mathrm{~mm}$, lower surface sparsely to scatteredly hairy, scatteredly covered with glandular hairs (sometimes only present towards the leaf margin), domatia absent, venation triplinerved, nerves 6-9 per side, looping and closed near margin. Inflorescences racemes, single, erect; peduncle $10-15$ by $1-2 \mathrm{~mm}$ long; axes densely to sparsely hairy; bracts triangular, $1.5-3$ by $0.8-1 \mathrm{~mm}$, persistent to caducous, margin entire, apex caudate, densely to sparsely hairy; bracteoles absent. Flowers: pedicels densely hairy; sepals basally connate, persistent, densely to sparsely hairy outside, glabrous inside, 


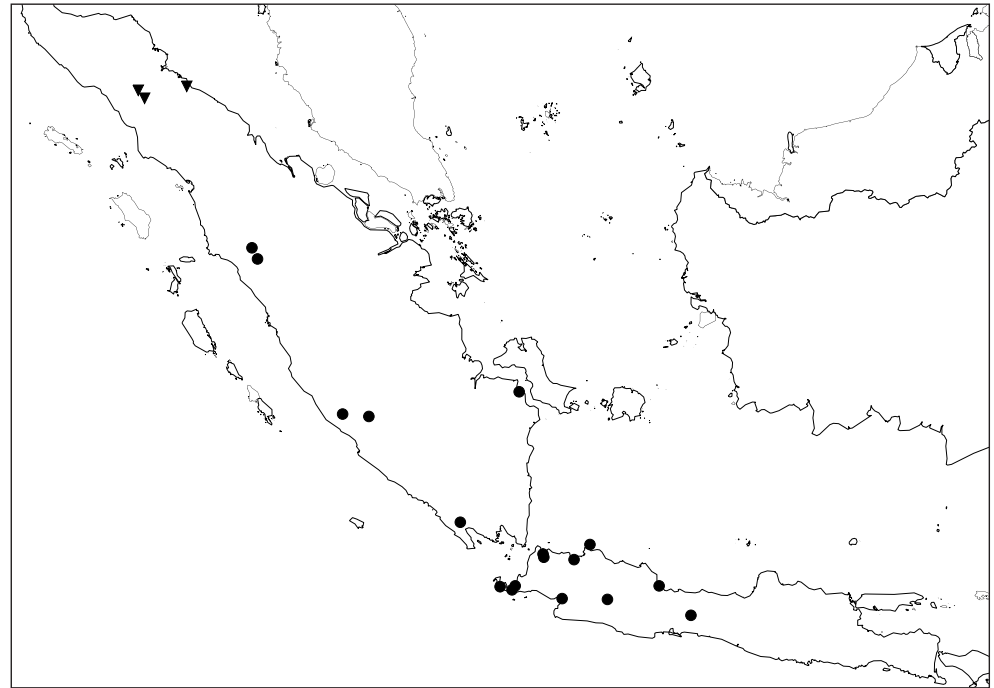

Map 3. Distribution of Mallotus blumeanus Müll.Arg. (•), M. sphaerocarpus (Miq.) Müll.Arg. (文).

glandular hairs absent, margin entire, apex acute. Staminate inflorescences 7.5-20 $\mathrm{cm}$ long, nodes per branch 33-45, with 6-8 flowers per bract. Staminate flowers 3-3.3 mm diam.; pedicels 1.5-7 mm long; calyx (rarely 2- or) 3-lobed, lobes ovate to elliptic, $1.8-2.5$ by $1-1.5 \mathrm{~mm}$; stamens $28-40$, glabrous, filaments $0.25-1.5 \mathrm{~mm}$ long, flattened, free, thecae ovoid, $0.1-0.2$ by $0.1-0.2 \mathrm{~mm}$, connective umbrella-like; pistillode absent. Pistillate inflorescences $8.5-16 \mathrm{~cm}$ long, nodes per branch 19-70, with 1 flower per bract. Pistillate flowers 3-4 mm diam; pedicels $1.3-2 \mathrm{~mm}$ long; calyx $3-5$-lobed, lobes triangular to narrowly triangular, $2.5-3$ by $1.2-1.8 \mathrm{~mm}$; ovary $1.8-2$ by $1.5-1.8 \mathrm{~mm}, 3$-locular, densely hairy, glandular hairs absent; style $0.2-0.5$ mm long; stigmas $2-3$ by $1-1.5 \mathrm{~mm}$. Fruits drupes, ellipsoid, $10-12$ by $4-4.4 \mathrm{~mm}$, surface smooth, sparsely hairy, glandular hairs rarely present; wall: woody part c. 0.1 mm thick, endocarp forming a thick fleshy layer around the seeds; column absent. Seeds lenticular, $5.5-7$ by $5-7$ by $3-4 \mathrm{~mm}$, surface rugose, dull, black.

Distribution - Sumatra, Java.

Habitat \& Ecology - In primary or old secondary and riparian forest; on ferrosolloam or limestone. Altitude 10-1000 m. Flowering and fruiting throughout the year.

Vernacular names - Sumatra: Entatau, kijak tukau, medang tekuku. Java: Ki tendjo, ki tengi (Sundanese); daon kajuole, hantap batu, pingku.

Notes - 1. Mallotus blumeanus can be easily confused with M. sphaerocarpus, but these two species differ in their indument and fruits. Mallotus blumeanus has brown yellow hairs, usually appressed (yellow and erect in $M$. sphaerocarpus) ellipsoid and densely hairy fruits (spheroid and sparsely hairy in M. sphaerocarpus).

2. Airy Shaw (1982) mentions that M. blumeanus occurs in Sulawesi and the Lesser Sunda Islands. This is probably based on, among other collections, Schmutz 4133 (L), from Flores (Lesser Sunda Islands). However, this is a misidentification, in Kulju et al. (2007) it is determined as M. cf. cumingii Müll.Arg. 


\section{Mallotus brevipetiolatus Gage - Map 4}

Mallotus brevipetiolatus Gage (1922) 242; Ridl. (1924) 286; Airy Shaw (1968) 393; Welzen et al. (2000) 97. - Lectotype (selected here): Ridley 15222 (holo K; iso BO, SING), Peninsular Malaysia, Perlis, Bukit Lagi.

Shrubs to small trees, up to $8 \mathrm{~m}$ high, dioecious. Outer bark smooth. Indumentum composed of simple, tufted and rarely peltate hairs and yellow to orange glandular hairs. Stipules triangular, $2.5-3.5$ by $1.3-2.2 \mathrm{~mm}$, early caducous, margin subentire, apex acute, scatteredly hairy. Leaves opposite; petiole $2-6$ by $1-2 \mathrm{~mm}$, scatteredly hairy to glabrous; blade elliptic to obovate, $4-21.5$ by $2.2-8.1 \mathrm{~cm}$, length/width ratio $2.5-3.8$, chartaceous, base shallowly emarginate to cuneate, margin denticulate to crenulate, with glandular teeth (located in a minute notch), apex acuminate to caudate, upper surface glabrous, sparsely to scatteredly covered with glandular hairs, extrafloral nectaries inconspicuous, touching the midrib to marginal in lower half, 1-3 per side, $2-8.5 \mathrm{~mm}$ from margin, elliptic to orbicular, $0.6-1.2$ by $0.2-0.4 \mathrm{~mm}$, lower surface glabrous, scatteredly covered with glandular hairs, domatia absent, venation pinnate, nerves $8-16$ per side, looping and closed near margin. Inflorescences racemes, single, erect; peduncle $2-12$ by $0.9-1.2 \mathrm{~mm}$; axes sparsely hairy; bracts scatteredly hairy to glabrous, deltoid or triangular, $1-1.7$ by $0.8-1.4 \mathrm{~mm}$, persistent, margin subentire, apex acute, scatteredly hairy to glabrous; bracteoles absent. Flowers: pedicels scatteredly hairy; sepals free, persistent, margin entire, apex acute, scatteredly hairy to glabrous outside, glabrous inside, with glandular hairs outside. Staminate inflorescences 7-22 $\mathrm{cm}$ long, nodes per branch 9-68, with 6-9 flowers per bract. Staminate flowers 3.5-5 $\mathrm{mm}$ diam., with sweet scent; pedicels $1.5-7 \mathrm{~mm}$ long; sepals 3 or 4 , elliptic to obovate, $2-3$ by $1.2-2 \mathrm{~mm}$; stamens $20-35$, filaments $1-3 \mathrm{~mm}$ long, free, glabrous, thecae ellipsoid, $0.3-0.4$ by $0.2-0.25 \mathrm{~mm}$, connnective not widened; pistillode absent. Pistillate

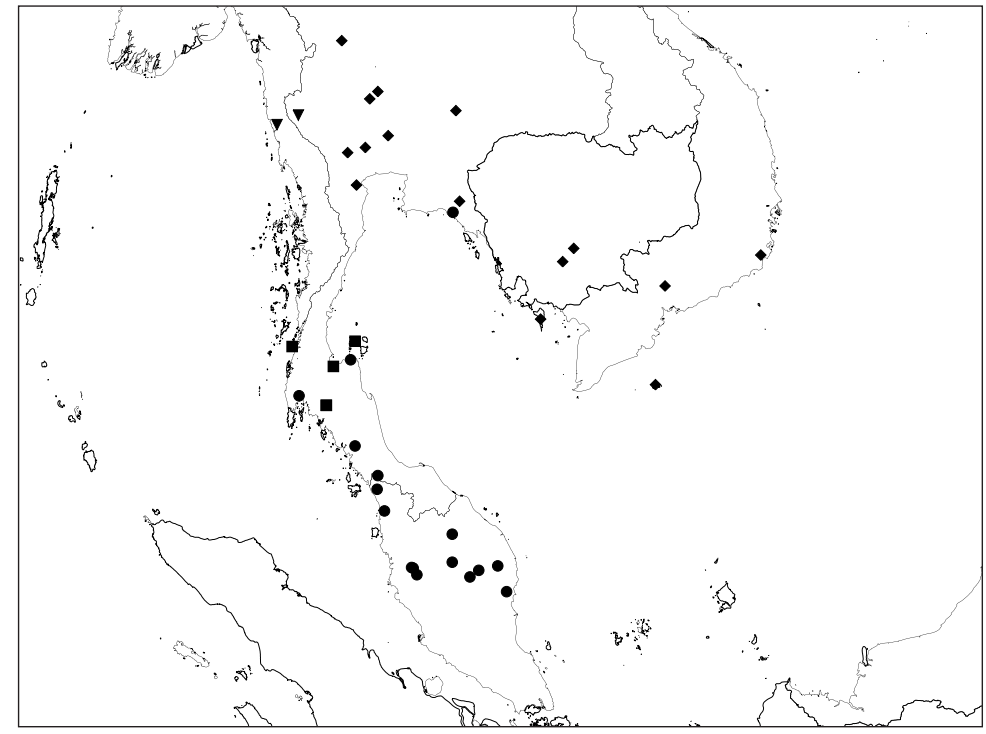

Map 4. Distribution of Mallotus brevipetiolatus Gage (•), M. coudercii (Gagnep.) Airy Shaw ( $\bullet$ ), M. hispidospinosus Welzen \& Chayam. ( $\mathbf{\nabla})$, M. hymenophyllus Airy Shaw ( $\mathbf{\square})$. 
inflorescences $4.7-10 \mathrm{~cm}$ long, nodes per branch 7-11, with 1 flower per bract. Pistillate flowers $2.5-3 \mathrm{~mm}$ diam.; pedicels $1-3 \mathrm{~mm}$ long; sepals 4-6, linear-triangular, $1.2-4.3$ by $0.5-0.8 \mathrm{~mm}$; ovary $1-1.4$ by $1.3-1.6 \mathrm{~mm}, 3$-locular, scatteredly hairy, densely covered with glandular hairs; style $0.5-0.7 \mathrm{~mm}$ long; stigmas $1.3-3.5$ by $0.8-1$ $\mathrm{mm}$. Fruits capsules, $5.8-8.9$ by $9-13 \mathrm{~mm}$, opening septicidally-loculicidally, surface spiny, scatteredly hairy, sparsely to scatteredly covered with glandular hairs, spines $50-70$, curved, $1.5-3$ by $0.2-0.3 \mathrm{~mm}$, apex hairy; wall $0.8-1 \mathrm{~mm}$ thick, sparsely hairy to glabrous inside; column $4.3-5$ by $4.8-7 \mathrm{~mm}$. Seeds \pm globose, $4.5-6.8$ by $3.8-5.9$ by $4.5-6 \mathrm{~mm}$, surface smooth, shiny, dark brown.

Distribution - Thailand, Peninsular Malaysia.

Habitat \& Ecology - In evergreen or mixed evergreen deciduous forest; on hill sides and shaded areas; on clay or limestone soil. Altitude 10-470 m. Flowering and fruiting throughout the year. Staminate flowers are visited by two genera of bees, Trigona and Apis dorsata.

\section{Mallotus calocarpus Airy Shaw - Map 1}

Mallotus calocarpus Airy Shaw (1968) 395; (1972) 294; Welzen et al. (2000) 98. - Type: Kerr 17965 (holo K; iso BK, L), Thailand, Chanthaburi, Kao Sabap.

Shrubs to small trees, 1-4 m high, dioecious. Indumentum composed of simple, tufted and stellately-tufted hairs and rarely glandular hairs. Stipules triangular to narrowly triangular, $5-6$ by $1.8-2.5 \mathrm{~mm}$, caducous, margin entire, apex acute, densely hairy outside, glabrous inside. Leaves opposite; petiole $4-19$ by $1-1.8 \mathrm{~mm}$, densely hairy; blade elliptic to obovate, $16-20$ by $5-9 \mathrm{~cm}$, length/width ratio $2.2-3.5$, chartaceous, base attenuate, margin denticulate to serrate, with glandular teeth, apex cuspidate to caudate, upper surface densely to sparsely hairy on midrib and nerves, larger extrafloral nectaries marginal in lower half, 3-6 nectaries per side, elliptic to orbicular, 2-3.8 by $0.7-1.5 \mathrm{~mm}$, smaller ones marginal from base to apex, $2-7$ per side, $5-14 \mathrm{~mm}$ from margin, elliptic to orbicular, $0.5-1$ by $0.3-0.5 \mathrm{~mm}$, lower surface sparsely to scatteredly hairy, glandular hairs rarely present (then scattered, near the margin or the petiole insertion), domatia absent, venation pinnate, nerves 8 or 9 per side, indistinctly looping and closed near margin or ending in margin. Inflorescences 1-3 together, erect; peduncle reduced; axes densely hairy; bracts triangular, persistent, margin entire, apex acute, densely hairy outside, glabrous inside. Flowers: pedicels densely hairy; sepals persistent, margin entire, apex acute. Staminate inflorescences reduced racemes, 2-2.3 $\mathrm{cm}$ long; nodes per branch c. 20, with 1 flower per bract; bracts triangular, 3.2-6 by $1.5-1.8 \mathrm{~mm}$; bracteoles absent. Staminate flowers 5-7 mm diam.; pedicels $3-5 \mathrm{~mm}$ long; sepals 3 , elliptic, $3.5-4$ by $1.5-2 \mathrm{~mm}$, free, densely hairy outside, scatteredly hairy to glabrous inside; stamens $34-50$, filaments $2.8-3 \mathrm{~mm}$ long, free, densely to sparsely hairy, thecae ellipsoid, $0.7-0.8$ by $0.2-0.25 \mathrm{~mm}$, glabrous, connective not widened; pistillode absent. Pistillate inflorescences reduced to 1 terminal flower, axis $1-1.5 \mathrm{~cm}$ long; bracts $3.3-6.2$ by $1.2-2 \mathrm{~mm}$; bracteoles triangular, $1-1.1$ by $0.9-1$ $\mathrm{mm}$, early caducous. Pistillate flowers 5-6 mm diam., sessile; calyx 5- or 6-lobed, lobes narrowly triangular, $5-5.5$ by $1-2 \mathrm{~mm}$, basally connate, densely hairy outside, scatteredly hairy to glabrous inside; ovary $2.5-3.5$ by $2.5-3 \mathrm{~mm}, 3$-locular, densely hairy; style $1.5-3 \mathrm{~mm}$ long; stigmas $6-10$ by $1-1.5 \mathrm{~mm}$. Fruits capsules, $15-17$ by 
20-22 mm, opening septicidally-loculicidally, surface smooth, densely hairy; wall 1-1.5 mm thick, glabrous inside; column $7-9$ by $3-6 \mathrm{~mm}$. Seeds \pm globose, $8.5-9$ by $7-8$ by $7-8 \mathrm{~mm}$, surface smooth, dull, light cream brown.

Distribution - Endemic to Thailand.

Habitat \& Ecology - In evergreen or deciduous rain forest; scattered along streams, in open areas. Altitude 50-300 m. Flowering: April to September; fruiting: October to January.

Note - See also the notes for M. mirus.

\section{Mallotus cauliflorus Merr. - Map 5}

Mallotus cauliflorus Merr. (1912b) 399. - Type: BS (M. Ramos) 13909 (holo PNH†; iso A, CAHUP,

K, US), Philippines, Luzon, Cagayan.

Woody, dioecious. Indumentum composed of simple and tufted hairs and yellow to orange glandular hairs. Stipules narrowly triangular, $18-30$ by $8-10 \mathrm{~mm}$, persistent, margin entire, apex acute, densely hairy, glabrescent. Leaves opposite; petiole 35-90 by $2-2.5 \mathrm{~mm}$, scatteredly hairy to glabrous; blade elliptic to obovate, $16-31$ by $6-12$ $\mathrm{cm}$, length/width ratio $2.3-2.5$, coriaceous, base rounded, obtuse to cuneate, margin entire to slightly repand, with glandular teeth, apex acuminate to caudate, upper surface glabrous, sparsely covered with glandular hairs, extrafloral nectaries at the axils between midrib and nerves, from base to apex, 1-20 per side, 3-60 mm from margin, elliptic to orbicular, $0.5-1$ by $0.5-0.8 \mathrm{~mm}$, lower surface sparsely hairy to subglabrous, densely covered with glandular hairs, domatia sometimes present, venation triplinerved, nerves 10-15 per side, looping and closed near margin. Inflorescences axillary, ramiflorous or cauliflorous racemes, 1-3 together, erect; axes sparsely hairy, with 1 flower per bract;

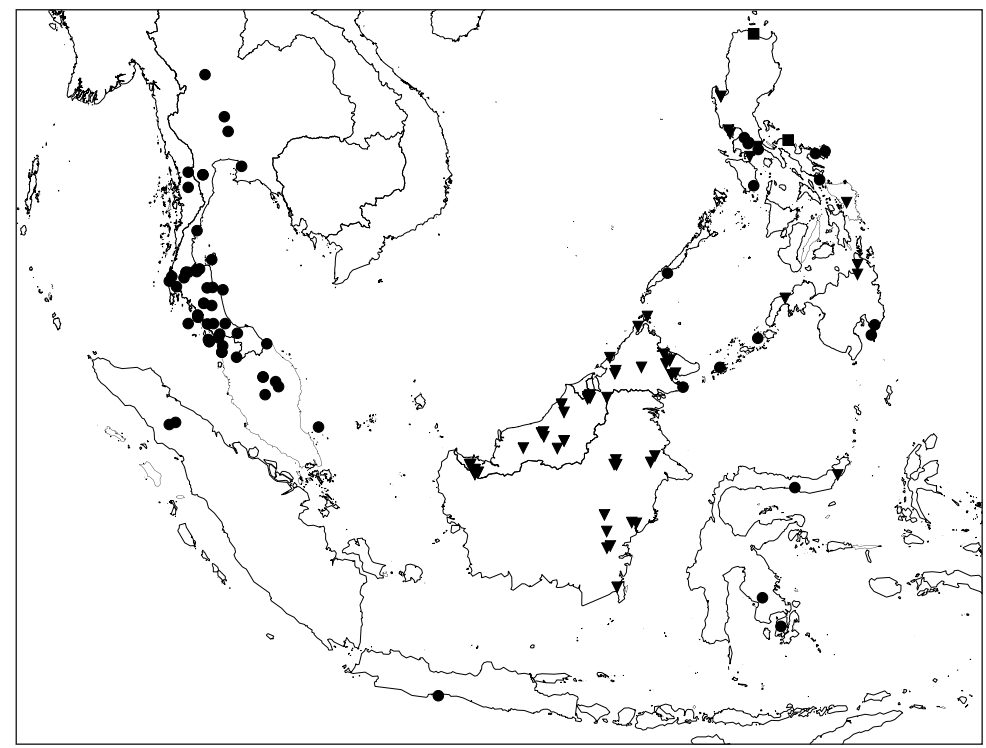

Map 5.Distribution of Mallotus cauliflorus Merr.(অ), M. leucocalyx Müll.Arg.(•), M. minimifructus S.E.C. Sierra $(\boldsymbol{\nabla})$. 
bracts triangular, margin subentire, densely hairy outside, glabrous inside; bracteoles absent. Flowers: pedicels scatteredly hairy; sepals 3, persistent, margin entire, apex acute, sparsely hairy outside, glabrous inside, with glandular hairs. Staminate inflorescences up to c. $10 \mathrm{~cm}$ long; peduncle 12-28 mm long; nodes per branch 36-60; bracts $0.6-0.9$ by $0.4-0.6 \mathrm{~mm}$, apex acute. Staminate flowers c. $3 \mathrm{~mm}$ diam.; pedicels $2.5-5 \mathrm{~mm}$ long; sepals 3 , elliptic, $2.5-3$ by $1.5-2 \mathrm{~mm}$, free; stamens $35-42$, glabrous, filaments $0.5-2.8 \mathrm{~mm}$ long, free, thecae ellipsoid, $0.2-0.3$ by $0.2-0.25 \mathrm{~mm}$, connective umbrella-like; pistillode absent. Pistillate inflorescences $7.5-12.5 \mathrm{~cm}$ long; peduncle $28 \mathrm{~mm}$ long; nodes per branch c. 13; bracts 3-4 by $1.5-2 \mathrm{~mm}$, apex acute. Pistillate flowers 3-4 mm diam.; pedicels $0.2-0.4 \mathrm{~mm}$ long; calyx 3-lobed, lobes triangular, $4-5$ by $1-3 \mathrm{~mm}$, basally connate; ovary $2-2.5$ by $2-2.5 \mathrm{~mm}, 3$-locular, sparsely hairy, densely covered with glandular hairs; style $0.3-0.5 \mathrm{~mm}$ long; stigmas $3-4$ by $0.8-1$ mm. Fruits capsules, 9-11 by 9-11 mm, opening septicidally-loculicidally, surface spiny, sparsely hairy, densely covered with glandular hairs; spines 90-120, curved, $0.5-1$ by $0.1-0.2 \mathrm{~mm}$, sparsely hairy; wall c. $0.8 \mathrm{~mm}$ thick, glabrous inside; column $3.5-5$ by $1.5-2.5 \mathrm{~mm}$. Seeds globose, $4.5-5$ by $4-4.5$ by $4-4.5 \mathrm{~mm}$, surface smooth, dull, light brown.

Distribution - Endemic to the Philippines.

Habitat \& Ecology - Altitude c. $80 \mathrm{~m}$. Flowering and fruiting: January to December.

\section{Mallotus claoxyloides (F. Muell.) Müll.Arg. - Map 2}

Mallotus claoxyloides (F. Muell.) Müll.Arg. (1865) 192; Airy Shaw (1966) 42; (1980c) 165; P.I. Forst. (1999) 460, 493. - Echinocroton claoxyloides F. Muell. (1858) 32. - Echinus claoxyloides (F. Muell.) Baill. (1866) 315, incl. var. genuina Baill., nom. inval. - Type: F. von Mueller s.n. ('Hill \& Mueller') (holo MEL barcode 708383; iso, GH, K), Australia, Queensland, Moreton, Brisbane River.

Rottlera (Plagianthera?) affinis Baill. (1858) 36, t. 19, f. 29-31,424, non Hassk. (1842). - Lectotype (designated here): Verreaux 592 (holo P; iso P), Australia.

Echinus claoxyloides (F. Muell.) Baill. var. cordata Baill. (1866) 315. - Mallotus claoxyloides (F. Muell.) Müll.Arg. var. cordatus (Baill.) Airy Shaw (1980d) 232. - Lectotype (P.I. Forster, 1999): Beckler s.n. (holo MEL barcode 515956 n.v.; iso P n.v.), Australia, New South Wales, Richmond River.

Mallotus claoxyloides (F. Muell.) Müll.Arg. var. glabratus Domin (1927) 888. - Type: Dietrich 524 (holo PR; iso AD, CANB, L, MEL), Australia, Queensland, S Kennedy, Port Mackay.

Shrubs to small trees, $0.5-8 \mathrm{~m}$ high, dioecious or rarely monoecious. Indumentum composed of simple and stellately-tufted hairs and yellow to orange glandular hairs. Stipules triangular to linear-triangular, $1.8-6$ by $0.6-1 \mathrm{~mm}$, persistent to caducous, margin entire, apex acute, scatteredly hairy to glabrous. Leaves opposite to rarely alternate; petiole $2-60$ by $0.8-1 \mathrm{~mm}$, glabrescent; blade elliptic to obovate, $4-17$ by $2.5-9 \mathrm{~cm}$, length/width ratio 1.6-2.1, (sub)coriaceous, base rounded, obtuse, cuneate to shallowly emarginate, margin entire, (denticulate), dentate to crenate, with glandular teeth, apex rounded to acuminate, upper surface sparsely hairy to glabrous on midrib and nerves, extrafloral nectaries marginal in lower half, 1-3(-5) per side, 1-2 mm from margin, elliptic to orbicular, $0.5-1$ by $0.4-0.6 \mathrm{~mm}$, lower surface sparsely hairy, glabrescent, sparsely covered with glandular hairs, domatia sometimes present, venation inconspicuously triplinerved, nerves 7 or 8 per side, indistinctly looping or ending 
in margin. Inflorescences single, erect; axes densely to sparsely hairy; bracts narrowly triangular, persistent, apex acute, margin entire, densely hairy outside, scatteredly hairy inside; bracteoles narrowly triangular $0.5-1$ by $0.2-0.3 \mathrm{~mm}$, early caducous. Flowers: pedicels densely hairy; sepals free, persistent, margin entire, apex acuminate, densely hairy outside, glabrous inside, with glandular hairs outside. Staminate inflorescences reduced racemes, 3.5-4 cm long; peduncle 7-20 mm long; nodes per branch 3-5, with 6-10 flowers per bract; bracts $1.5-4.5$ by $0.3-1 \mathrm{~mm}$, apex acuminate. Staminate flowers 5-6.5 mm diam.; pedicels $2-5 \mathrm{~mm}$ long; sepals ( 2 or) 3 , elliptic to obovate, $4-5$ by $1.8-2.2 \mathrm{~mm}$; stamens $29-45$, filaments $1.8-5.5 \mathrm{~mm}$ long, free, glabrous, thecae ellipsoid, $0.2-0.3$ by $0.2-0.25 \mathrm{~mm}$, hairy or glabrous, connective widened; pistillode present. Pistillate inflorescences umbel-like, $0.8-1.5 \mathrm{~cm}$ long, usually with an extra flower near the base of the axes; peduncle 3-6 mm long; nodes per branch 1 or 2, with (1-) 3-5 flowers per bract; bracts $1.5-2.8$ by $0.2-0.4 \mathrm{~mm}$, apex acute. Pistillate flowers 4-6 $\mathrm{mm}$ diam.; pedicels 3-10 $\mathrm{mm}$ long; sepals 3 or 4 , triangular, $3-5$ by $0.8-1.2 \mathrm{~mm}$; ovary $1.8-2$ by $1.5-1.8 \mathrm{~mm}, 3$-locular, densely hairy, scatteredly covered with glandular hairs; style $0.5-1 \mathrm{~mm}$, stigmas $3-3.5$ by $1.2-1.5 \mathrm{~mm}$. Fruits capsules, 6-8.5 by 11-13 mm, opening septicidally-loculicidally, surface spiny, densely hairy, scatteredly covered with glandular hairs; spines $65-80$, curved, $1-1.3$ by $0.1-0.2 \mathrm{~mm}$, sparsely hairy; wall $0.8-1 \mathrm{~mm}$ thick, glabrous inside; column $4-5$ by $3-5 \mathrm{~mm}$. Seeds globose, $5-5.5$ by $4-5$ by $4-5 \mathrm{~mm}$, surface smooth, dull, light-brown.

Distribution - New Guinea, Australia.

Habitat \& Ecology - Locally common in edges of evergreen forests, semi-evergreen vine-thickets and mangroves; in araucarian notophyll and microphyll vineforest; along dried gullies and creeks; on sedimentary, coral sand, limestone, rocky, sandy loam or basalt soil. Altitude 20-500 m. Flowering and fruiting throughout the year.

Notes - 1. Mallotus claoxyloides can be confused with M. megadontus P.I. Forst. and $M$. ficifolius. From $M$. megadontus and $M$. ficifolius it differs in the presence of thin filaments (thick in M. megadontus and M.ficifolius), whitish indument (yellowish in $M$. megadontus and $M$. ficifolius), narrowly emarginate, rounded, obtuse to acute leaf base (obtuse, cuneate to attenuate in M. megadontus, cordate rounded to cuneate in M.ficifolius), petioles $2-6 \mathrm{~cm}$ long (3-5 cm in M. megadontus, 7-8 cm in M. ficifolius), leaf venation pinnate or triplinerved (pinnate in $M$. megadontus, triplinerved in $M$. ficifolius), fruits sparsely hairy (sparsely hairy in M. megadontus, densely hairy in M. ficifolius).

2. See also the note for M. attenuatus.

3. According to label information, the fresh leaves emit a strong smell of benzaldehyde.

8. Mallotus connatus M. Aparicio, spec. nov. - Fig. 4; Map 6

Folia opposita non valde inaequalia, nectaria extrafloralia marginalia vel ubique, nervi 4-6 per latere. Inflorescentiae bracteae anguste triangulares ad trilobatae. Flores staminales: filamenta basi connata, pistillodium adest. Flores pistillata: stylus 2-4 mm longus. Fructus spinosus. - Typus: Mahyar et al. 1197 (holo L; iso A, SING), Borneo, Kalimantan, Sintang, HPH Km 86.

Mallotus moritzianus auct. non Müll.Arg.: Slik, Priyono \& Welzen (2000) 60, f. 35.

Mallotus rufidulus auct. non (Miq.) Müll.Arg.: Airy Shaw (1981) 328, pro Borneo. 
Shrubs to small trees, up to $9 \mathrm{~m}$ high; dioecious or rarely monoecious. Indumentum composed of simple, tufted and stellately-tufted hairs and yellow to orange glandular hairs. Stipules linear-triangular, 3-7 by $0.5-1 \mathrm{~mm}$, early caducous, margin entire, apex acute, densely to sparsely hairy. Leaves opposite; petiole $5-70$ by $1-1.8 \mathrm{~mm}$, densely to sparsely hairy; blade ovate to elliptic, $7-27$ by $5-14 \mathrm{~cm}$, length/width ratio $1.5-3$, membranaceous to chartaceous, base rounded, obtuse to cuneate, margin entire, apex caudate, upper surface densely to sparsely hairy on midrib and nerves, extrafloral nectaries marginal throughout blade, up to 20 nectaries per side, $1-8 \mathrm{~mm}$ from margin, sometimes also all over the blade, elliptic to orbicular, $0.2-0.5$ by $0.2-0.5 \mathrm{~mm}$, lower surface sparsely to scatteredly hairy, sparsely covered with glandular hairs, domatia absent, venation triplinerved, nerves 4-6(-9) per side, looping and closed near margin. Inflorescences racemes, 1 (or 2) together, erect, unisexual or rarely bisexual; peduncle $20-60$ by $0.5-1.5 \mathrm{~mm}$; axes sparsely hairy; bracts narrowly triangular or trilobed, $3-5$ by $0.8-3 \mathrm{~mm}$, persistent, margin entire, apex acute, densely hairy; bracteoles narrowly to linear-triangular, $2-3.8$ by $0.3-0.5 \mathrm{~mm}$, early caducous. Flowers: pedicels sparsely to densely hairy; sepals margin entire, apex acute, densely hairy outside, with scattered glandular hairs, glabrous inside. Staminate inflorescences $8-22 \mathrm{~cm}$ long, nodes per branch 11-13, with 5-13 flowers per bract. Staminate flowers 3-4 mm diam.; pedicels 1-4 mm long; calyx 3- or 4-lobed, lobes ovate, $3-5$ by $0.4-0.6 \mathrm{~mm}$; stamens $45-60$, glabrous, filaments $3.5-5 \mathrm{~mm}$ long, connate in lower half, thecae ovoid, $0.2-0.3$ by 0.2-0.25 mm, connective widened; pistillode present. Pistillate inflorescences 16-21 $\mathrm{cm}$ long, nodes per branch 3-10, with 1 flower per bract. Pistillate flowers 4-4.2 mm diam.; pedicels 3-7 mm long; sepals 4 or 5, triangular, 5-6.5 by 1-2 mm, free; staminodes rarely present; ovary $2.5-3$ by $2.5-3 \mathrm{~mm}, 3$-locular, densely hairy, green, scatteredly covered with glandular hairs; style $2-4 \mathrm{~mm}$ long; stigmas $3-7$ by $0.8-1 \mathrm{~mm}$.

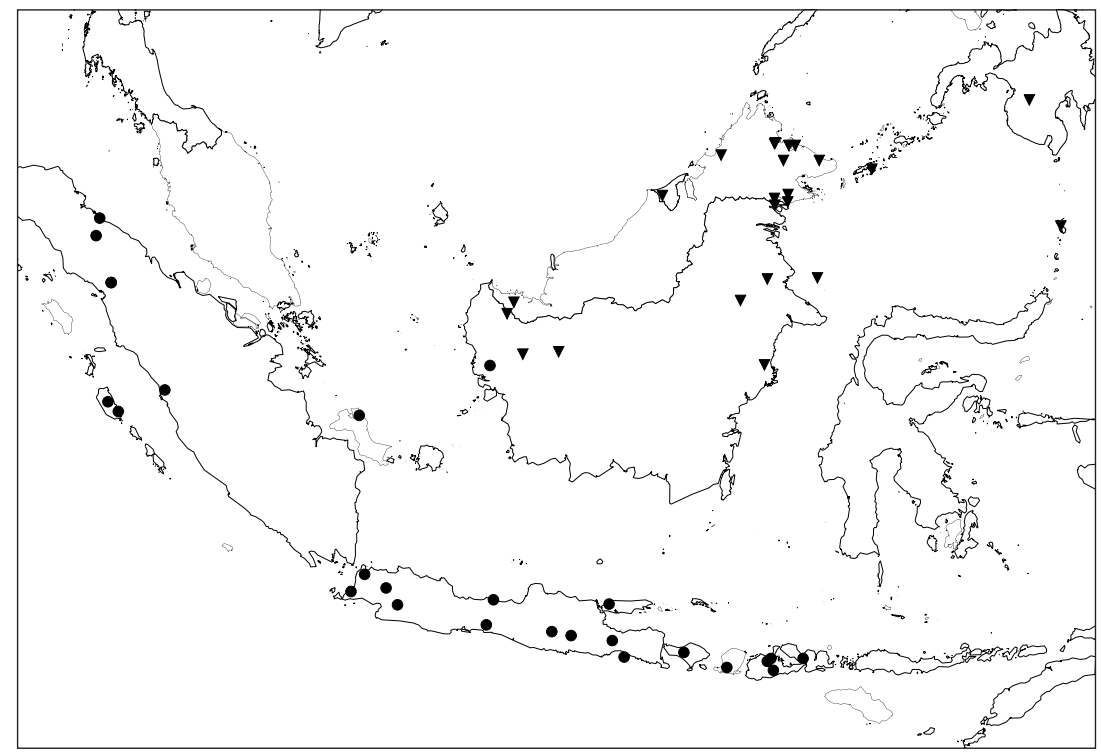

Map 6. Distribution of Mallotus connatus M. Aparicio , M. rufidulus (Miq.) Müll.Arg. 


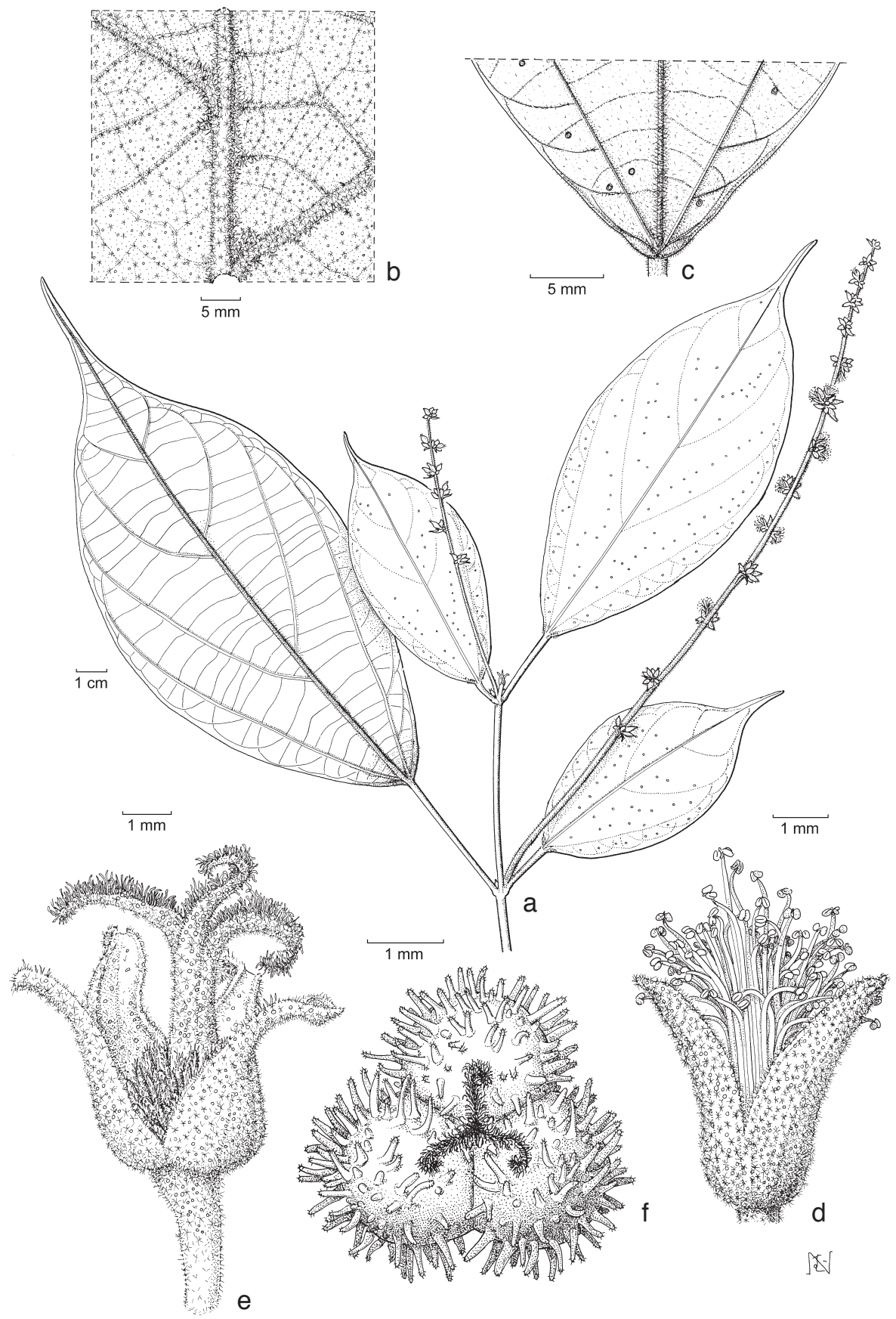

Fig. 4. Mallotus connatus M. Aparicio. a. Habit with staminate inflorescences; b. detail of lower leaf surface with glandular hairs; c. base of upper leaf surface with extrafloral nectaries; d. staminate flower; e. pistillate flower; f. fruit (a-d: Kostermans 21548; e: Elmer 20747; f: Ambriansyah et al. B883; all. L). 
Fruits capsules, $10-15$ by $10-16 \mathrm{~mm}$, pale green to green, opening septicidally-loculicidally, surface spiny, densely hairy, scatteredly covered with glandular hairs; spines $200-250, \pm$ straight, $1.5-3$ by $0.2-0.3 \mathrm{~mm}$, densely hairy; wall $0.8-1.2 \mathrm{~mm}$ thick, glabrous inside; column $7-8$ by $7-8 \mathrm{~mm}$. Seeds \pm globose, $8.2-10$ by $8.2-10$ by $8.2-10$ mm, surface smooth, dull, brown.

Distribution - Borneo, Philippines.

Habitat \& Ecology - Primary and secondary forest, along river sides; on alluvial to clay-loam soil. Altitude 30-200 m. Flowering and fruiting: March to November.

Vernacular names - Borneo: Kemantah rimba.

Note - Mallotus connatus can easily be confused with M. rufidulus. The main differences are found in leaf and floral characters: Leaves drying green to green-brown (brown in M.rufidulus); stipules linear-triangular (narrowly triangular in M.rufidulus); pistillate inflorescences with entire or trilobed bracts (trilobed in M. rufidulus), pistillate flower buds ellipsoid (ovoid in M. rufidulus), connate filaments (free to slightly connate at the base in $M$.rufidulus), styles $2-4 \mathrm{~mm}$ long $(0-1(-2)) \mathrm{mm}$ in $M$. rufidulus), stigmas $0.8-1 \mathrm{~mm}$ thick (1-1.3 mm in M.rufidulus). Besides differences in morphology they also differ in geographical distribution; M. connatus is found in Borneo and the Philippines, while M. rufidulus is found in Sumatra, Java, Borneo and Lesser Sunda Islands.

\section{Mallotus coudercii (Gagnep.) Airy Shaw - Map 4}

Mallotus coudercii (Gagnep.) Airy Shaw (1966) 42; (1968) 381. - Coelodiscus coudercii Gagnep. (1923) 49. - Lectotype (selected here): Thorel 2027 (holo P; iso NY), Cambodia, Me-Kong, Oudong.

Mallotus spodocarpus Airy Shaw (1968) 383; (1972) 304; Welzen et al. (2000) 109. - Type: Put 2169 (holo K; iso BK, L), Thailand, Northern, Nakhon Sawan, Hua Wai.

Coelodiscus glabriusculus auct. non Kurz (1877): Gagnep. (1925) 372, p.p.: Godefroy s.n.(P, barcode P00432571), Cambodia; Pierre s.n. (K, barcodes K000252842-43; P, barcodes P00432573-75), Vietnam; Harmand s.n. (P, barcode P00432570), Vietnam.

Shrubs, up to $0.6 \mathrm{~m}$ high, dioecious. Indumentum composed of simple and stellatelytufted hairs and light yellow glandular hairs. Stipules triangular to linear-triangular, 6-12 by $0.3-0.8 \mathrm{~mm}$, persistent to caducous, margin entire, apex acute, scatteredly hairy. Leaves opposite or rarely alternate; petiole $3-60$ by $1.5-2 \mathrm{~mm}$, sparsely to scatteredly hairy; blade ovate to obovate, $3-18$ by $3-10.5 \mathrm{~cm}$, length/width ratio $1-1.9$, membranaceous to chartaceous, base shallowly emarginate, rounded to obtuse, margin entire to (shallowly) dentate, with glandular teeth, apex rounded, acute to cuspidate, upper surface sparsely hairy on midrib and nerves, extrafloral nectaries rarely present, inconspicuous, marginal in lower half, $1-4$ per side, $0.2-0.5 \mathrm{~mm}$ from margin, elliptic to orbicular, $0.2-0.3$ by $0.2-0.3 \mathrm{~mm}$, lower surface scatteredly hairy and glandular hairy, domatia sometimes present, venation triplinerved, nerves 4-8 per side, looping and closed near margin. Inflorescences single, erect; peduncle 3.5-17 mm; axes densely to sparsely hairy; bracts triangular to narrowly triangular, $1.5-3.5$ by $0.5-1.8$ $\mathrm{mm}$, persistent, margin entire, apex caudate, sparsely to scatteredly hairy outside, glabrous inside; bracteoles narrowly triangular, $1.5-3$ by $0.2-0.3 \mathrm{~mm}$, persistent to caducous. Flowers: pedicels 1-2 mm long, densely hairy; sepals persistent, margin entire, apex acute, glabrous inside, densely to sparsely hairy outside, glabrous inside, 
with glandular hairs outside. Staminate inflorescences reduced racemes, $1.5-3 \mathrm{~cm}$ long, nodes per branch 3-5, with 4-9 flowers per bract. Staminate flowers $4-5 \mathrm{~mm}$ diam.; pedicels $0.5-1.3 \mathrm{~mm}$ long; sepals 3 or 4 , elliptic to obovate, $1.4-1.6$ by $1.1-1.3 \mathrm{~mm}$, free; stamens $25-29$, filaments $2-3 \mathrm{~mm}$ long, free, hairy, thecae ellipsoid, $0.2-0.3$ by $0.2-0.25 \mathrm{~mm}$, glabrous, connective widened; pistillode present. Pistillate inflorescences umbel-like, $6.3-12 \mathrm{~cm}$ long, usually with an extra flower near the base of the axes; nodes per branch 1 or 2, with (1-) 4-7 flowers per bract. Pistillate flowers 3-3.5 mm diam.; pedicels $1-4 \mathrm{~mm}$ long; calyx 4-lobed, lobes narrowly triangular, $1.3-1.5$ by $0.9-1.2$ $\mathrm{mm}$, basally connate; ovary $1.2-1.3$ by $1.5-1.6 \mathrm{~mm}$, (2- or) 3 - (or 4-)locular, densely hairy, sparsely covered with glandular hairs; style $0.6-3 \mathrm{~mm}$ long; stigmas $2-2.2$ by $0.3-0.5 \mathrm{~mm}$. Fruits capsules, $4.5-8$ by $9-12 \mathrm{~mm}$, opening septicidally-loculicidally, surface smooth, densely hairy, sparsely covered with glandular hairs; wall c. $0.1 \mathrm{~mm}$ thick, glabrous inside; column $3-4.5$ by $1.2-2.5 \mathrm{~mm}$. Seeds \pm globose, $3.5-5$ by $3.5-4.2$ by $3.6-4.2 \mathrm{~mm}$, surface rugose, dull, dark brown.

Distribution - Thailand, Cambodia, Vietnam.

Habitat \& Ecology - In mixed, deciduous, bamboo forests, in open areas; on limestone, volcanic or basalt soil. Altitude $20-250 \mathrm{~m}$. Flowering: January to July.

Note - Mallotus coudercii differs from M. glabriusculus in the mostly absent extrafloral nectaries (always present in M. glabriusculus), absence or presence of domatia (always present in M. glabriusculus), leaves usually drying greenish (usually drying brownish in M. glabriusculus), densely hairy filaments (glabrous or scatteredly hairy in M. glabriusculus), smooth fruits (spiny in M. glabriusculus).

\section{Mallotus darbyshirei Airy Shaw - Map 7}

Mallotus darbyshirei Airy Shaw (1980a) 597. - Type: Darbyshire \& Hoogland 7956 (holo BRI; iso A, L), Papua New Guinea, Aitape, Aiserap.

Shrubs, up to $4 \mathrm{~m}$ high, dioecious; branches hollow. Indumentum composed of simple and stellately-tufted hairs and dark red glandular hairs. Stipules triangular, c. 7 by 3 $\mathrm{mm}$, early caducous, margin entire, apex acute, densely hairy outside, glabrous inside. Leaves opposite; petiole $18-54$ by $1.8-3 \mathrm{~mm}$, densely to sparsely hairy; blade elliptic to obovate, $17-34.5$ by $7-18 \mathrm{~cm}$, length/width ratio $1.9-2.4$, chartaceous, base obtuse to cuneate, margin entire, undulate, apex caudate, upper surface sparsely to scatteredly hairy on midrib and nerves, extrafloral nectaries along the midrib, 20-30 per side, also all over the blade, elliptic to orbicular, $0.6-0.8$ by $0.5-0.6 \mathrm{~mm}$, lower surface sparsely hairy, scatteredly covered with glandular hairs (sometimes only present near the leaf margin), domatia absent, venation inconspicuously triplinerved, nerves 5 or 6 per side, looping and closed near margin. Staminate inflorescences unknown. Pistillate inflorescences umbels, 1 or 2 together, erect, $3.8-4.5 \mathrm{~cm}$ long, axes densely hairy; peduncle $25-27$ by $1.2-1.5 \mathrm{~mm}$; nodes per branch 1 or 2 , with 3 or 4 flowers per bract; bracts triangular, c. 2 by $1 \mathrm{~mm}$, margin entire, apex acute; bracteoles absent. Pistillate flowers 5-6 mm diam.; pedicels 6-10 $\mathrm{mm}$ long, densely hairy; sepals 3 , triangular to narrowly triangular, $4-4.5$ by c. $1.5 \mathrm{~mm}$, free, early caducous, margin entire, apex acute, densely hairy outside, glabrous inside; ovary $3-4$ by $3-4 \mathrm{~mm}$, 3-locular, densely hairy, without glandular hairs; stigmas sessile, 3-4 by $0.8-1 \mathrm{~mm}$. Fruits capsules, c. 9 by $14 \mathrm{~mm}$, green, opening septicidally-loculicidally, surface spiny, densely hairy, without glandular 


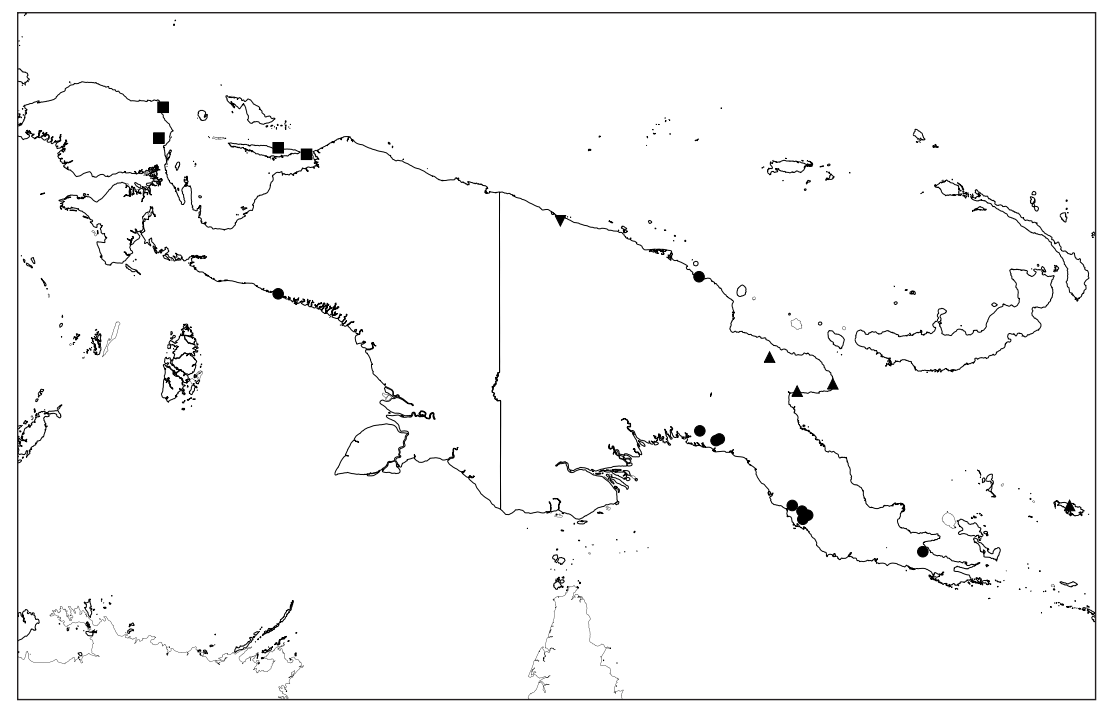

Map 7. Distribution of Mallotus darbyshirei Airy Shaw ( $\mathbf{\nabla})$, M. didymochryseus Airy Shaw (•), M. lauterbachianus (Pax \& K. Hoffm.) Pax \& K. Hoffm. (४), M. macularis Airy Shaw (ם).

hairs; spines $500-600, \pm$ straight, $2.5-3$ by $0.2-0.3 \mathrm{~mm}$, densely hairy; wall $0.8-1$ $\mathrm{mm}$ thick, densely hairy inside; column c. 6 by $5 \mathrm{~mm}$. Seeds \pm globose, c. 6.5 by 4.5 by $4.5 \mathrm{~mm}$, surface smooth, dull, brown.

Distribution - Endemic to New Guinea.

Habitat \& Ecology - On gentle slope. Altitude c. 15 m. Fruiting: June.

Vernacular name - Wommollo (Wapi).

\section{Mallotus decipiens Müll.Arg. - Map 8; Plate 1d}

Mallotus decipiens Müll.Arg. (1865) 194; Hook.f. (1887) 434; Airy Shaw (1972) 303. - Lectotype (selected here): Wallich 8009 (holo G-DC, IDC microfiche 800, the sheet with the field number 2130 and male infl.; iso K-W, IDC microfiche 7394), Myanmar, Mon Prov., banks of Ataran River ('2130, Myanmar, ripae Attran'), see note 1.

Coelodiscus eriocarpoides Kurz [(1873) 244, nom. nud.] (1877) 392. - Type: Parish s.n. (CAL n.v., fide Hook.f., 1887: 434), Myanmar, Upper Taninthayi Prov. ('Myanmar, Upper Tenasserim'), see note 2 .

Mallotus cuneatus Ridl. (1911) 181. - Mallotus resinosus (Blanco) Merr. var. cuneatus (Ridl.) N.P. Balakr. \& Chakrab. (1991) 39. - Type: Ridley 15190 (holo SING; iso K), Peninsular Malaysia, Perlis, Bukit Lagi, see note 3.

Coelodiscus muricatus auct. non. (Wight) Gagnep.: Gagnep. (1925) 369, excl. basion.: minime: Eberhardt 3258 (K), Vietnam, Lang Son; Bon 2574 (P), Vietnam, Hanoi; Pételot 1277 (P, UC), Vietnam, Tonkin, Cho Ganh; Pételot 1417 (UC), Vietnam, Tonkin, Cho Ganh; Pételot 1418 (UC), Vietnam, Tonkin, Cho Ganh; Pierre s.n. (P), Cambodia, Mt Schrall; all others not seen.

Shrubs, 0.5-4 m high, dioecious. Outer bark slightly rough. Indumentum composed of simple and tufted hairs and yellow to orange glandular hairs. Stipules triangular, 2-4 by 1-1.8 mm, caducous, margin entire, apex acute, scatteredly hairy outside, glabrous inside. Leaves opposite; petiole $2-24$ by $0.9-1.4 \mathrm{~mm}$, sparsely hairy; blade obovate, 
$2.8-20$ by $1.6-12 \mathrm{~cm}$, length/width ratio $1.9-2.4$, membranaceous to chartaceous, base obtuse to cuneate, margin denticulate, dentate (specially towards the apex) to crenate, with glandular teeth, apex cuspidate to caudate, upper surface scatteredly hairy to glabrous on midrib and nerves, extrafloral nectaries marginal in lower half, 1 or 2 per side, $1.5-3 \mathrm{~mm}$ from margin, elliptic to orbicular, $0.8-2.2$ by $0.6-0.8 \mathrm{~mm}$, lower surface sparsely hairy, densely (to sparsely) covered with glandular hairs, domatia sometimes present, venation triplinerved, nerves 6-8 per side, looping and closed near margin or ending in margin. Inflorescences racemes, single, erect; peduncle $8-32$ by $0.2-1 \mathrm{~mm}$; axes densely to sparsely hairy; bracts triangular, persistent, margin entire to subentire, apex acute to acuminate, densely to sparsely hairy outside, glabrous inside; bracteoles sometimes present, triangular, $0.2-0.3$ by $0.2-0.3 \mathrm{~mm}$, caducous, margin entire. Flowers: pedicels densely to sparsely hairy; sepals persistent, margin entire, apex acute, sparsely hairy and with glandular hairs outside, glabrous inside. Staminate inflorescences 7-16 cm long, nodes per branch 22-41, with 2-7 flowers per bract; bracts $1-2$ by $0.9-1 \mathrm{~mm}$. Staminate flowers $2.5-4 \mathrm{~mm}$ diam.; pedicels $1.8-2.2 \mathrm{~mm}$ long; sepals 3 , elliptic to obovate, $1.5-2.2$ by $0.7-1.5 \mathrm{~mm}$, free; stamens $22-32$, filaments $1.3-3 \mathrm{~mm}$ long, free, glabrous, thecae ovoid, $0.2-0.3$ by $0.2-0.25$ $\mathrm{mm}$, usually glabrous, connective widened; pistillode present. Pistillate inflorescences $6.8-16 \mathrm{~cm}$ long, nodes per branch 13-36, with 1 flower per bract. Pistillate flowers $0.8-1 \mathrm{~mm}$ diam.; pedicels $0.3-1 \mathrm{~mm}$ long; calyx 3-lobed, lobes triangular, 1.5-1.7 by $0.8-1 \mathrm{~mm}$; ovary $0.5-0.6$ by $0.5-0.6 \mathrm{~mm}$, 3-locular, sparsely hairy, scatteredly covered with glandular hairs; style $0.2-0.5 \mathrm{~mm}$ long; stigmas $0.7-1$ by c. $1 \mathrm{~mm}$. Fruits capsules, 3-4 by 4-6 mm, pale light green, opening septicidally-loculicidally, surface spiny, sparsely hairy, sparsely covered with glandular hairs; spines 150-180, curved, $0.5-0.7$ by $0.2-0.25 \mathrm{~mm}$, scatteredly hairy; wall c. $0.2 \mathrm{~mm}$ thick, glabrous inside;

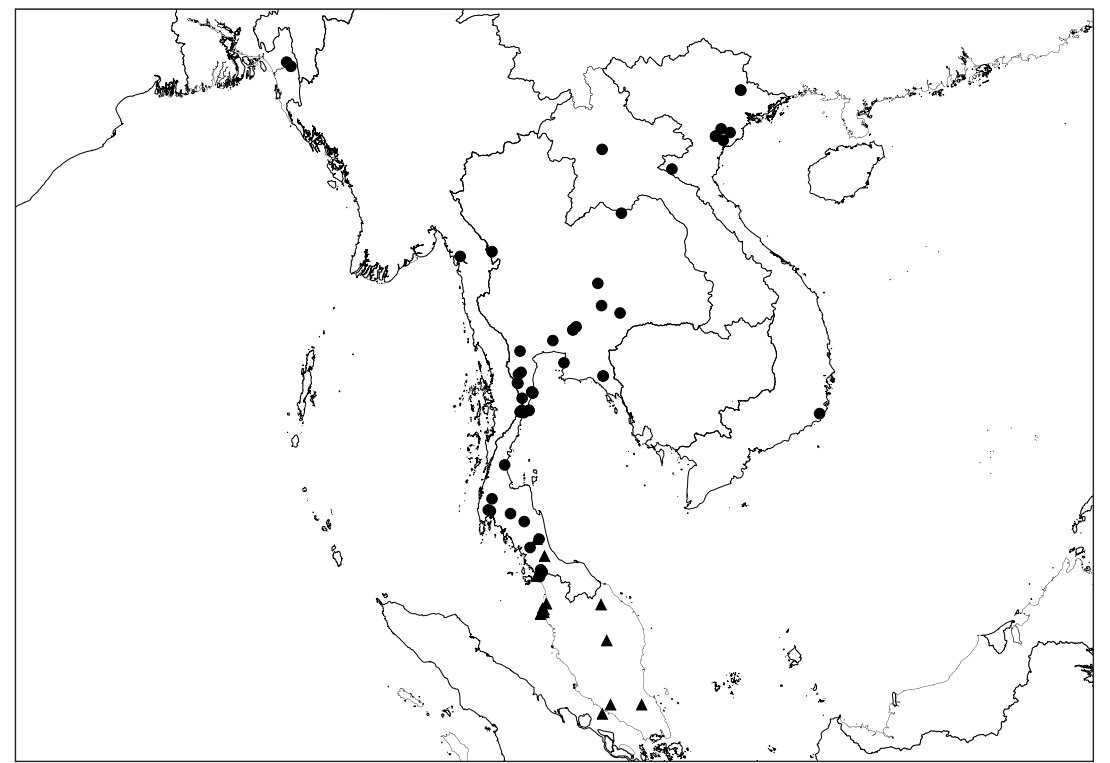

Map 8. Distribution of Mallotus decipiens Müll.Arg. (•), M. montanus (Müll.Arg.) Airy Shaw (()). 
column $2.9-3.1$ by $2.9-3.1 \mathrm{~mm}$. Seeds \pm globose, $3.6-3.8$ by $3.1-3.2$ by c. $3.2 \mathrm{~mm}$, surface smooth, shiny, brown-grey.

Distribution - Bangladesh, Myanmar, Laos, Vietnam, Thailand, Peninsular Malaysia (northern).

Habitat \& Ecology - Locally common in very degraded, seasonal, evergreen hardwood forests with bamboo, deciduous dipterocarp-oak and pine forests, dry secondary scrub; in shaded areas, along roadsides, or on steep slopes; on granite, limestone, calcareous rock, sandstone, or shale bedrock soil. Altitude: sea level up to $800 \mathrm{~m}$. Flowering and fruiting throughout the year.

Vernacular name - Vietnam: Cáy duoi rúng.

Notes - 1. Müller Argoviensis (1865) described Mallotus decipiens on "Wall.! In hb. DC." However, there are two gatherings in Wallich's Numerical List: 7725 and 8009 , curiously both labelled "Attran, 17 May 1827", to which 7725 adds 'ripae', i.e. riverbanks, and 8009 the field number 2130. In G-DC there is a sheet labelled "2073, Attran R. 17 May 1827" with a female plant. The 7725 sheet in K-W has both male and female inflorescences, which indicates a mixed collection, possibly of field numbers 2073 and 2130.

2. The type, place of deposit, and identity of Coelodiscus eriocarpoides Kurz are accepted here on the base of the remarks made by Hooker f. (1887: 434). The brief diagnosis corresponds to our concept of M.decipiens.

3. Balakrishnan \& Chakrabarty (1991) recognized M. cuneatus at the varietal level, mentioning that it differs from $M$. resinosus by the presence of triplinerved leaf venation and hairy capsules (pinnate venation and densely glandular capsules in M. resinosus). However, there are more characters that separate them: Extrafloral nectaries with at least one pair always present on the nerves (below the nerves in M.resinosus), 6-8 nerves ( $8-12$ in M. resinosus), smaller fruits, 3-4 by 4-6 mm (larger fruits, $6-8$ by $8-15$ in $M$. resinosus), presence of short spines, $0.5-0.7$ by $0.2-0.3$ (longer, $1-2.2$ by $0.3-0.5$ in M.resinosus).

\section{Mallotus didymochryseus Airy Shaw - Map 7}

Mallotus didymochryseus Airy Shaw (1966) 40. - Type: NGF (C.T. White) 8255 (holo K; iso CANB, L), Papua New Guinea, Lawes.

Small to large trees, 8-28 m high, dioecious. Outer bark shallowly longitudinal fissured. Indumentum composed of simple, stellately-tufted and stellate hairs (on petioles), glandular hairs absent. Stipules triangular to narrowly triangular, $1-1.8$ by $0.4-0.6$ $\mathrm{mm}$, early caducous, margin entire, apex acute, densely hairy. Leaves opposite; petiole $25-80$ by $2-3 \mathrm{~mm}$, densely to sparsely hairy; blade ovate, $12-23.5$ by $9.5-22 \mathrm{~cm}$, length/width ratio 1.1-1.4, chartaceous, base cordate to rounded, margin entire to shallowly repand, with indistinct glandular teeth, apex rounded, acute to acuminate, upper surface densely hairy on midrib and nerves, extrafloral nectaries marginal from base to apex, $10-35$ per side, $1-6 \mathrm{~mm}$ from margin, elliptic to orbicular, $0.2-0.7$ by $0.2-0.5$ $\mathrm{mm}$, lower surface sparsely hairy, domatia absent, venation triplinerved, nerves 6 or 7 per side, indistinctly looping or ending in margin. Inflorescences racemes, single, erect; peduncle $6-45$ by $1.5-2 \mathrm{~mm}$; axes densely hairy; bracts triangular to narrowly triangular, margin entire, apex acute, densely hairy, early caducous; bracteoles absent. 
Flowers: pedicels densely hairy; sepals free, persistent, margin entire, apex acute, densely hairy outside, glabrous inside. Staminate inflorescences $20-22 \mathrm{~cm}$ long, nodes per branch $35-47$, with $5-7$ flowers per bract; bracts $1-2.2$ by $0.4-0.6 \mathrm{~mm}$. Staminate flowers 5-7 mm diam.; pedicels 3.5-4 mm long; sepals 3 or 4, ovate to elliptic, $2.5-3$ by $1.5-1.8 \mathrm{~mm}$; stamens $60-90$, filaments $1-3 \mathrm{~mm}$ long, free, glabrous, white, thecae ellipsoid, $0.2-0.3$ by $0.2-0.25 \mathrm{~mm}$, hairy, pale light cream, connective widened; pistillode present. Pistillate inflorescences 5-12 cm long, nodes per branch $8-30$, with 1 flower per bract; bracts $1-3$ by $0.5-0.9 \mathrm{~mm}$. Pistillate flowers $3-5 \mathrm{~mm}$ diam.; pedicels $5-8 \mathrm{~mm}$ long; sepals $4-6$, triangular, $2.5-3$ by $0.8-1 \mathrm{~mm}$; ovary $1.6-1.8$ by $1.5-1.7 \mathrm{~mm}$, 2-locular, densely hairy; style $0.2-0.4 \mathrm{~mm}$ long; stigmas $2.3-2.7$ by c. $2 \mathrm{~mm}$. Fruits tardily dehiscent capsules, opening loculicidally, 10-12.5 by $12-17 \mathrm{~mm}$, orange yellow, surface wrinkled, densely hairy; wall c. $0.5 \mathrm{~mm}$ thick, glabrous inside; column $8-9$ by $3-3.2 \mathrm{~mm}$. Seed $s \pm$ globose, $6.5-7.5$ by $7-8$ by $6-7$ $\mathrm{mm}$, surface slightly rugose, dull, whitish cream.

Distribution - Endemic to New Guinea.

Habitat \& Ecology - Scattered in the understorey of seasonally dry evergreen rain forest or in the upper tier of mixed primary or secondary alluvial lowland or swamp forest (periodically flooded to $1 \mathrm{~m}$ deep); on forest edges. Altitude: sea level up to $580 \mathrm{~m}$. Flowering and fruiting: November to July.

Vernacular names - New Guinea: Indonesia: Kamuretjia (Tarie); Papua New Guinea: Tivamunana (Maya); Okaibu.

Notes -1 . All the fruits studied were closed (even the ripe ones) and lacked the characteristic sutures present of dehiscent Mallotus species.

2. Mallotus didymochryseus can be distinguished from $M$. trinervius by the sandpapery texture of the upper surface of the leaves (soft in M.trinervius), 10-35 marginal extrafloral nectaries ( 5 or 6 in M.trinervius), cordate to rounded leaf base (rounded to cuneate in M.trinervius) and the tardily dehiscent, small, $10-12.5$ by $12-17 \mathrm{~mm}$, orange yellow fruits (dehiscent, large, 17-22.5 by 24-29 mm, brown ochre in M.trinervius).

\section{Mallotus dispar (Blume) Müll.Arg. - Map 9}

Mallotus dispar (Blume) Müll.Arg. (1865) 191 (excl. var. psiloneurus Müll.Arg. = M. resinosus (Blanco) Merr.) in DC. (1866) 971; Airy Shaw (1968) 380; (1972) 303; Whitmore (1973) 115; Airy Shaw (1975) 169; Welzen et al. (2000) 99. - Rottlera dispar Blume (1826) 608. - Mallotus dispar (Blume) Müll.Arg. var. lasioneurus Müll.Arg. (1865) 191, nom. inval. - Type: Blume s.n. (L), Java, Kuripan.

Shrubs to small trees, 2-10 m high, dioecious or rarely monoecious. Indumentum composed of simple and tufted hairs and yellow to orange glandular hairs. Stipules narrowly triangular, $5-6$ by $1.2-1.5 \mathrm{~mm}$, persistent to caducous, margin entire, apex acute, densely hairy outside, sparsely hairy inside. Leaves opposite; petiole $3-50$ by $0.8-1.3 \mathrm{~mm}$, densely hairy; blade ovate to obovate, $5-20$ by $4-9 \mathrm{~cm}$, length/width ratio 2.1-2.5, membranaceous to chartaceous, base rounded, obtuse to cuneate, margin denticulate, dentate to crenate, with glandular teeth, apex acuminate to caudate, upper surface glabrous, extrafloral nectaries marginal in lower half, rarely along the midrib, 1 or 2 (or 3) per side, 1.5-7(-12) mm from margin, elliptic to orbicular, $0.5-2$ by $0.5-1 \mathrm{~mm}$, lower surface sparsely hairy, sparsely covered with glandular hairs, domatia 


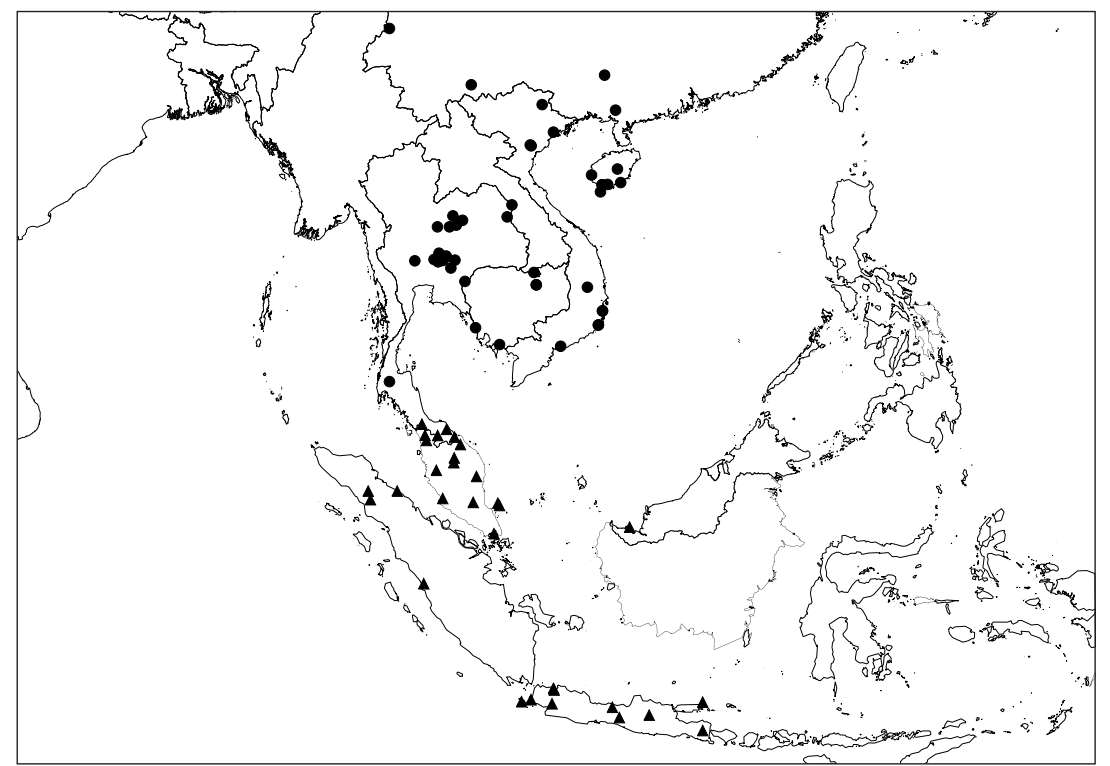

Map 9. Distribution of Mallotus dispar (Blume) Müll.Arg. (\), M. lanceolatus (Gagnep.) Airy Shaw (๑).

sometimes present, venation triplinerved, nerves 7-9 per side, looping and closed near margin. Inflorescences racemes, single, erect, unisexual to rarely bisexual; peduncle $15-30$ by $0.5-0.8 \mathrm{~mm}$ long; axes densely hairy; bracts narrowly to linear-triangular, margin entire, apex acute, densely hairy outside, scatteredly hairy to glabrous inside; bracteoles absent. Flowers: pedicels densely to sparsely hairy; sepals free, persistent, margin entire, apex acute, densely hairy outside, scatteredly hairy to glabrous inside, with glandular hairs outside. Staminate inflorescences $6-12 \mathrm{~cm}$ long, nodes per branch 12-18, with 1-4 flowers per bract; bracts $3-5.5$ by $0.7-1.3 \mathrm{~mm}$. Staminate flowers $3.5-4 \mathrm{~mm}$ diam.; pedicels $2-4 \mathrm{~mm}$ long; sepals 3 or 4 , elliptic to obovate, $1.8-2.3$ by $0.5-1.3 \mathrm{~mm}$, apex acute; stamens $28-50$, glabrous, filaments $1.5-2.5 \mathrm{~mm}$ long, free, thecae ovoid, $0.2-0.3$ by $0.2-0.25 \mathrm{~mm}$, connective widened; pistillode present. Pistillate inflorescences 5-12 cm long, nodes per branch 6-9, with 1 flower per bract; bracts $3.5-6$ by $0.8-1.7 \mathrm{~mm}$; bracteoles absent. Pistillate flowers $2.5-4 \mathrm{~mm}$ diam.; pedicels $1.5-2 \mathrm{~mm}$ long; sepals $3-5$, narrowly triangular, $3-4$ by $0.8-1.2 \mathrm{~mm}$; staminodes rarely present; ovary $0.8-1.5$ by $1.8-2 \mathrm{~mm}, 3$-locular, densely hairy, sparsely covered with glandular hairs; style $0.7-1.3 \mathrm{~mm}$ long; stigmas $3.5-6$ by $1.5-2 \mathrm{~mm}$. Fruits capsules, $8-12$ by $9.5-13 \mathrm{~mm}$, light green, opening septicidally-loculicidally, surface spiny, sparsely hairy, sparsely covered with glandular hairs; spines 60-80, curved, $2-4$ by $0.1-0.2 \mathrm{~mm}$, sparsely hairy; wall $0.7-1 \mathrm{~mm}$ thick, glabrous inside; column $3-5$ by $3-5 \mathrm{~mm}$. Seed \pm globose, $4-5$ by $4-5$ by $4-5 \mathrm{~mm}$, surface smooth, shiny, brown, aril or arillode absent.

Distribution - From Thailand to Peninsular Malaysia, Sumatra, Java. 
Habitat \& Ecology - Locally in evergreen or coastal forest mixed with lowland dipterocarp forest. On clay, limestone or brown soil, on cliffs. Altitude: sea level up to $600 \mathrm{~m}$. Flowering and fruiting throughout the year.

Note - Mallotus dispar differs from M. minimifructus in the presence of 1 or 2 (or 3 ) pairs of extrafloral nectaries, with al least one pair always present on the nerves, (2-7 pairs below the nerves in M. minimifructus).

\section{Mallotus eximius Airy Shaw - Map 10}

Mallotus eximius Airy Shaw (1978a) 400. - Type: KEP FRI (Loh) 13517 (holo K; iso A, L, SING), Peninsular Malaysia, Ulu Trengganu.

Small trees, 2-6 m high, dioecious. Indumentum composed of simple, tufted and stellately-tufted hairs and light yellow to orange glandular hairs. Stipules narrowly triangular, $5-12$ by $1.5-5 \mathrm{~mm}$, early caducous, margin entire, hairy, apex acute, densely hairy outside, sparsely hairy inside. Leaves opposite; petiole $60-120$ by $2-3 \mathrm{~mm}$, densely hairy; blade ovate to elliptic, $12-48$ by $7-25 \mathrm{~cm}$, length/width ratio $1.7-2.6$, chartaceous, base sometimes peltate up to $5 \mathrm{~mm}$, cordate, rounded to obtuse, margin entire, apex caudate, upper surface densely hairy on midrib and nerves, extrafloral nectaries marginal throughout blade, up to 30 per side, 2-30 $\mathrm{mm}$ from margin, sometimes also all over the blade, elliptic to orbicular, $0.2-0.8$ by $0.2-0.5 \mathrm{~mm}$, lower surface sparsely hairy, sparsely covered with glandular hairs, domatia absent, venation triplinerved, nerves 7-9 per side, looping and closed near margin. Inflorescences racemes, single, erect; axes densely hairy; bracts persistent, densely hairy, margin entire or trilobed, apex acute; bracteoles sometimes present. Flowers: pedicels densely hairy; sepals connate

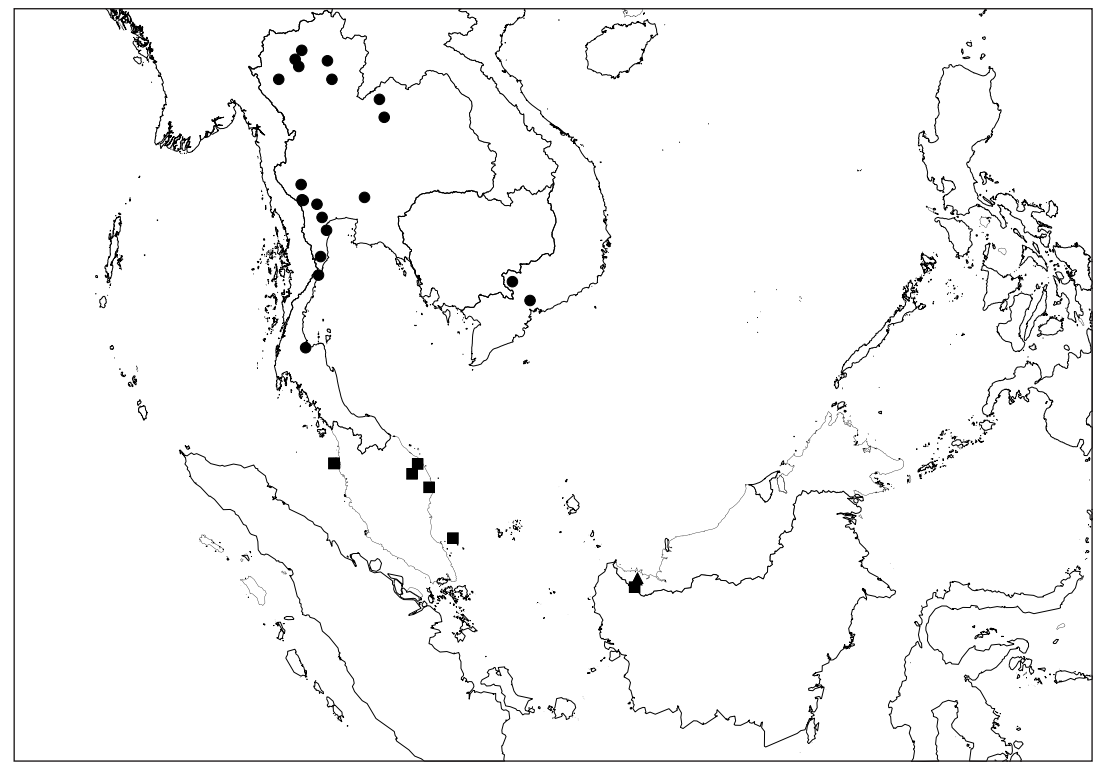

Map 10. Distribution of Mallotus eximius Airy Shaw (ם), M. longinervis M. Aparicio (४), M. pierrei (Gagnep.) Airy Shaw (•). 
in lower half, margin entire, apex acute, surfaces densely hairy, without glandular hairs. Staminate inflorescences $10-15 \mathrm{~cm}$ long; peduncle 10-20 mm long; nodes per branch $18-20$, with $4-6$ flowers per bract; bracts triangular, $2.8-3.2$ by $0.6-0.8 \mathrm{~mm}$; bracteoles entire or trilobed, $0.8-2$ by $0.3-0.4 \mathrm{~mm}$. Staminate flowers $4.8-5.2 \mathrm{~mm}$ diam.; pedicels 1-3 mm long; calyx 3-lobed, lobes triangular to narrowly triangular, $3-4.1$ by $1.8-2 \mathrm{~mm}$; stamens $30-35$, filaments $3-5 \mathrm{~mm}$ long, connate in lower half, hairy, thecae ovoid, $0.2-0.3$ by $0.2-0.25 \mathrm{~mm}$, subglabrous, connective not widened; pistillode present. Pistillate inflorescences $15-34 \mathrm{~cm}$ long, nodes per branch 6-12, with 1 flower per bract; bracts trilobed, 6-9 by $4-6 \mathrm{~mm}$; bracteoles absent. Pistillate flowers 6-8 mm diam.; pedicels 6-11 $\mathrm{mm}$ long; calyx 5- or 6-lobed, lobes triangular, $4.5-6$ by $3-4 \mathrm{~mm}$; ovary $3.2-4$ by $4-5 \mathrm{~mm}, 3$-locular, densely hairy, glandular hairs absent; style 2-3 mm long; stigmas $4-7$ by $1.2-1.4 \mathrm{~mm}$ long. Fruits capsules, 17-20 by $18-20 \mathrm{~mm}$, green to brown green, opening septicidally-loculicidally, surface spiny, densely hairy, without glandular hairs; spines c. $700, \pm$ straight, $1.5-3$ by $0.4-0.6 \mathrm{~mm}$, densely hairy, hairs forming a continuous layer; wall 1.7-2 mm thick, glabrous inside; column $12-13$ by $10-11 \mathrm{~mm}$. Seeds \pm globose, $10-12$ by $9-11$ by $9-11 \mathrm{~mm}$, surface smooth, dull, brown.

Distribution - Peninsular Malaysia, NW Borneo.

Habitat \& Ecology - Locally common in understorey of primary to secondary forests, scrubs, mostly found in disturbed sites, also on hillsides; on granite to limestone soil. Altitude 150-400 m. Flowering and fruiting: May to September.

Vernacular name - Borneo: Brati manuk (Dayak).

\section{Mallotus glabriusculus (Kurz) Pax \& K. Hoffm. - Map 11}

Mallotus glabriusculus (Kurz) Pax \& K. Hoffm. (1914) 162; Airy Shaw (1972) 303. - Coelodiscus glabriusculus Kurz (1877) 393; Gagnep. (1925) 372, p.p.: Pierre s.n. (P, barcode P00432568; K, barcode K000252584), Vietnam. - Lectotype (selected here): Kurz 1242 (holo CAL n.v.; iso K), Myanmar, Pegu, Palawa.

Mallotus clellandii Hook.f. (1887) 435; Airy Shaw (1968) 381; (1972) 302; Welzen et al. (2000) 98. - Type: Clelland s.n. (holo K, barcode K000187483), Myanmar, Pegu, Rangoon.

Mallotus tristis Pax \& K. Hoffm. (1914) 154. - Type: Meebold 7845 (holo B? †; iso K), Myanmar, Phaungbyin, Ober-Chindwin.

Shrubs, 1.5-3 m high, dioecious. Indumentum composed of simple and stellately-tufted hairs and light yellow glandular hairs. Stipules linear-triangular, $3-10$ by $0.5-1 \mathrm{~mm}$, persistent to caducous, margin entire, apex acute, scatteredly hairy to glabrous. Leaves opposite; petiole $5-75$ by $1-2 \mathrm{~mm}$, sparsely to scatteredly hairy; blade ovate, narrowly elliptic (to obovate), 6-24 by $3-10 \mathrm{~cm}$, length/width ratio $1.8-4$, chartaceous, base cordate, rounded to obtuse, margin entire to denticulate, with glandular teeth, apex (rounded to) acute to caudate, upper surface glabrous, extrafloral nectaries marginal in lower half, sometimes also at the axils between midrib and nerves, 1 or 2(-5) per side, $2.5-6 \mathrm{~mm}$ from margin, elliptic to orbicular, $0.3-1$ by $0.2-0.8 \mathrm{~mm}$, lower surface scatteredly hairy, scatteredly covered with glandular hairs, domatia present, venation triplinerved, nerves 4-8 per side, looping and closed near margin. Inflorescences single, erect; axes densely hairy; peduncle 3-10 mm long; bracts triangular to narrowly triangular to linear-triangular, $2-3$ by $0.3-0.8 \mathrm{~mm}$, persistent to caducous, margin entire, apex caudate, densely hairy outside, scatteredly hairy inside; bracteoles 


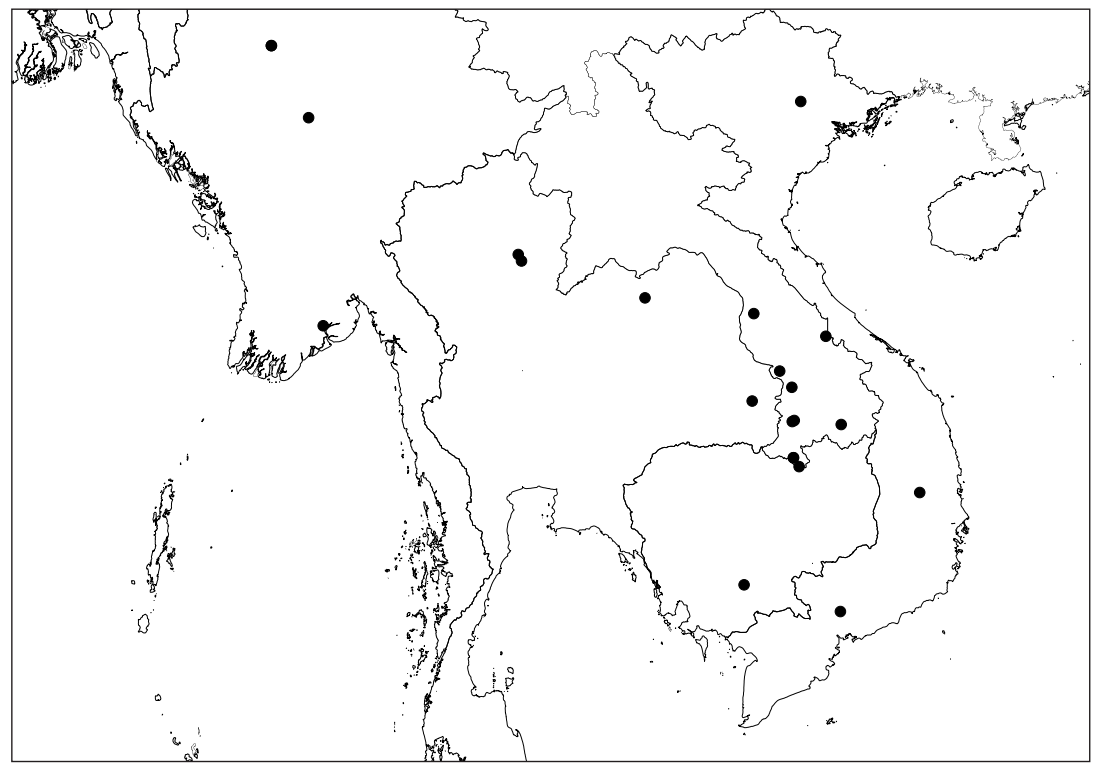

Map 11. Distribution of Mallotus glabriusculus (Kurz) Pax \& K. Hoffm.

narrowly triangular, $1.5-3$ by $0.2-0.4 \mathrm{~mm}$, persistent to caducous. Flowers: pedicels densely to sparsely hairy; sepals persistent, margin entire, apex acute, densely hairy outside, glabrous inside, with glandular hairs outside. Staminate inflorescences reduced racemes, 1-4 cm long; nodes per branch 4-8, with 3-7 flowers per bract. Staminate flowers 4.5-6 mm diam.; pedicels $0.8-3 \mathrm{~mm}$ long; sepals 3, ovate to elliptic, 3.5-5 by $2.5-3.5 \mathrm{~mm}$, free; stamens $35-55$, filaments $2-3 \mathrm{~mm}$ long, free, sometimes hairy, thecae ellipsoid, $0.6-0.8$ by $0.2-0.25 \mathrm{~mm}$, glabrous, connective widened; pistillode present. Pistillate inflorescences racemes or umbel-like, $1.5-5.2 \mathrm{~cm}$ long, usually with an extra flower on the base of the axes, nodes per branch 1 or 2, with (1-)4-7 flowers per bract. Pistillate flowers $2.5-4 \mathrm{~mm}$ diam.; pedicels $2-4.5 \mathrm{~mm}$ long; calyx 3- or 4lobed, lobes narrowly triangular, $3-4$ by $1.3-1.6 \mathrm{~mm}$, basally connate; ovary $1.3-1.5$ by $1.6-1.9 \mathrm{~mm}$, (2- or) 3-locular, densely hairy, scatteredly covered with glandular hairs; style $0.5-2 \mathrm{~mm}$ long; stigmas $3-4$ by $0.6-0.8 \mathrm{~mm}$. Fruits capsules, $7-13$ by 13-16 mm, reddish green, opening septicidally-loculicidally, surface spiny, densely to sparsely hairy, scatteredly covered with glandular hairs; spines 200-350, \pm straight, $3-5$ by $0.2-0.4 \mathrm{~mm}$, densely to sparsely hairy; wall $0.2-0.5(-0.8) \mathrm{mm}$ thick, glabrous inside; column $4.5-7$ by $2.5-5 \mathrm{~mm}$. Seeds \pm globose, $6.5-8$ by $3.8-5$ by $3-5 \mathrm{~mm}$, surface smooth, shiny, light to dark brown.

Distribution - Myanmar, Thailand, Laos, Cambodia, Vietnam.

Habitat \& Ecology - In mixed dry dipterocarp forest and degraded terrains; on sandy soil or shale bedrock. Altitude 60-457 m. Flowering and fruiting throughout the year.

Vernacular names - Laos: Hoon hi, kahn hayo nohk kaw (Lao); Khi hit.

Note - See also the note for M. coudercii. 


\section{Mallotus glomerulatus Welzen - Fig. 5; Map 1}

Mallotus glomerulatus Welzen in Welzen et al. (2004) 173. - Type: Koonthunthod, Spuntee \& Thetsna 524 (holo L; iso BKF), Thailand, Nakhon Phanom Prov., Ban Phaeng, Phu Langka National Park, Tat Pho Waterfall.

Shrubs, 1-1.5 m high, dioecious (or monoecious, according to label information). Indumentum composed of simple and tufted hairs, glandular hairs absent. Stipules triangular, $2-3.5$ by $1-2 \mathrm{~mm}$, early caducous, margin entire, apex acute, sparsely hairy outside, glabrous inside. Leaves opposite; petiole $6-15$ by $2.8-3.5 \mathrm{~mm}$, densely to sparsely hairy; blade elliptic, $9-27$ by $3.5-8.2 \mathrm{~cm}$, length/width ratio $3-3.8$, coriaceous, base attenuate, margin entire to crenate, with a single subapical tooth at each side, apex cuspidate to caudate, upper surface glabrous, larger extrafloral nectaries marginal in lower half, 3-5 nectaries per side, 1.5-2 mm from margin, elliptic to orbicular, 1-1.7 by $0.8-1.5 \mathrm{~mm}$, smaller ones marginal from base to apex, $8-10$ per side, $3-7 \mathrm{~mm}$ from margin, orbicular, $0.2-0.3$ by $0.2-0.3 \mathrm{~mm}$, lower surface scatteredly hairy to glabrous, domatia absent, venation pinnate, nerves 8-10 per side, looping and closed near margin. Inflorescences axillary or ramiflorous, erect, 1-3 together; peduncle absent; axes densely hairy; bracts narrowly triangular; bracteoles absent. Flowers: pedicels densely hairy; sepals persistent, margin entire, apex acute, densely hairy outside, glabrous inside. Staminate inflorescences with glomerules of flowers, $1 \mathrm{~cm}$ long; bracts 1-2.5 by $0.5-1 \mathrm{~mm}$. Staminate flowers $3.5-4 \mathrm{~mm}$ diam.; pedicels $3-5 \mathrm{~mm}$ long; sepals 3 or 4 , ovate, $2.5-3.5$ by $1.2-1.8 \mathrm{~mm}$, free; stamens $25-30$, glabrous, filaments $0.5-1$ $\mathrm{mm}$ long, free, thecae ellipsoid, $0.7-0.8$ by $0.2-0.25 \mathrm{~mm}$, connective not widened; pistillode present. Pistillate inflorescences reduced to 1-4 terminal flowers, ax is 1-1.5 cm long; bracts 4 or 5, 2.5-4 by 1.5-2 mm. Pistillate flowers 4-5 mm diam., sessile, calyx 5- or 6-lobed, lobes narrowly triangular, $3-5$ by $0.8-2 \mathrm{~mm}$, basally connate; ovary $1.8-2$ by $1.5-1.8 \mathrm{~mm}$, 3-locular, densely hairy; style $2-2.5 \mathrm{~mm}$ long; stigmas 4-5 by $0.7-1 \mathrm{~mm}$. Fruits capsules, $9-10$ by $13-14 \mathrm{~mm}$, opening septicidally-loculicidally, surface smooth, scatteredly hairy; wall c. $0.8 \mathrm{~mm}$ thick, glabrous inside; column $4-7$ by $2-3 \mathrm{~mm}$. Seeds \pm globose, $4-7$ by $3.3-6$ by $3.8-6 \mathrm{~mm}$, surface smooth, dull, light cream brown.

Distribution - Endemic to Thailand.

Habitat \& Ecology - In dry evergreen forest, in shade. Altitude 150-200 m. Flowering: May; fruiting: May to August.

Vernacular name - Mak lium.

\section{Mallotus hispidospinosus Welzen \& Chayam. - Map 4}

Mallotus hispidospinosus Welzen \& Chayam. (2001) 650,f. 1. - Mallotus spec.nov. 1 Welzen et al. (2000) 108. - Mallotus spinihispidus Welzen \& Chayam. in Smitinand (2001) 342, nomen.

- Type: P. Sangkhachand 821 (holo BK n.v.), Thailand, Southwestern, Kanchanaburi, Pilok.

Shrubs, up to $1 \mathrm{~m}$ high, dioecious. Outer bark smooth. Indumentum composed of simple and tufted hairs and yellow glandular hairs. Stipules linear-triangular, $3-6.2$ by 0.3-0.5 mm, caducous, margin entire, apex acute, glabrous. Leaves opposite; petiole $10-21.5$ by $0.8-1 \mathrm{~mm}$, densely hairy; blade ovate to elliptic, $4-18.5$ by $3.2-7.1 \mathrm{~cm}$, length/width ratio 2.3-3.4, membranaceous, base shallowly emarginate, margin dentate, with glandular teeth, apex caudate, upper surface glabrous, extrafloral nectaries 


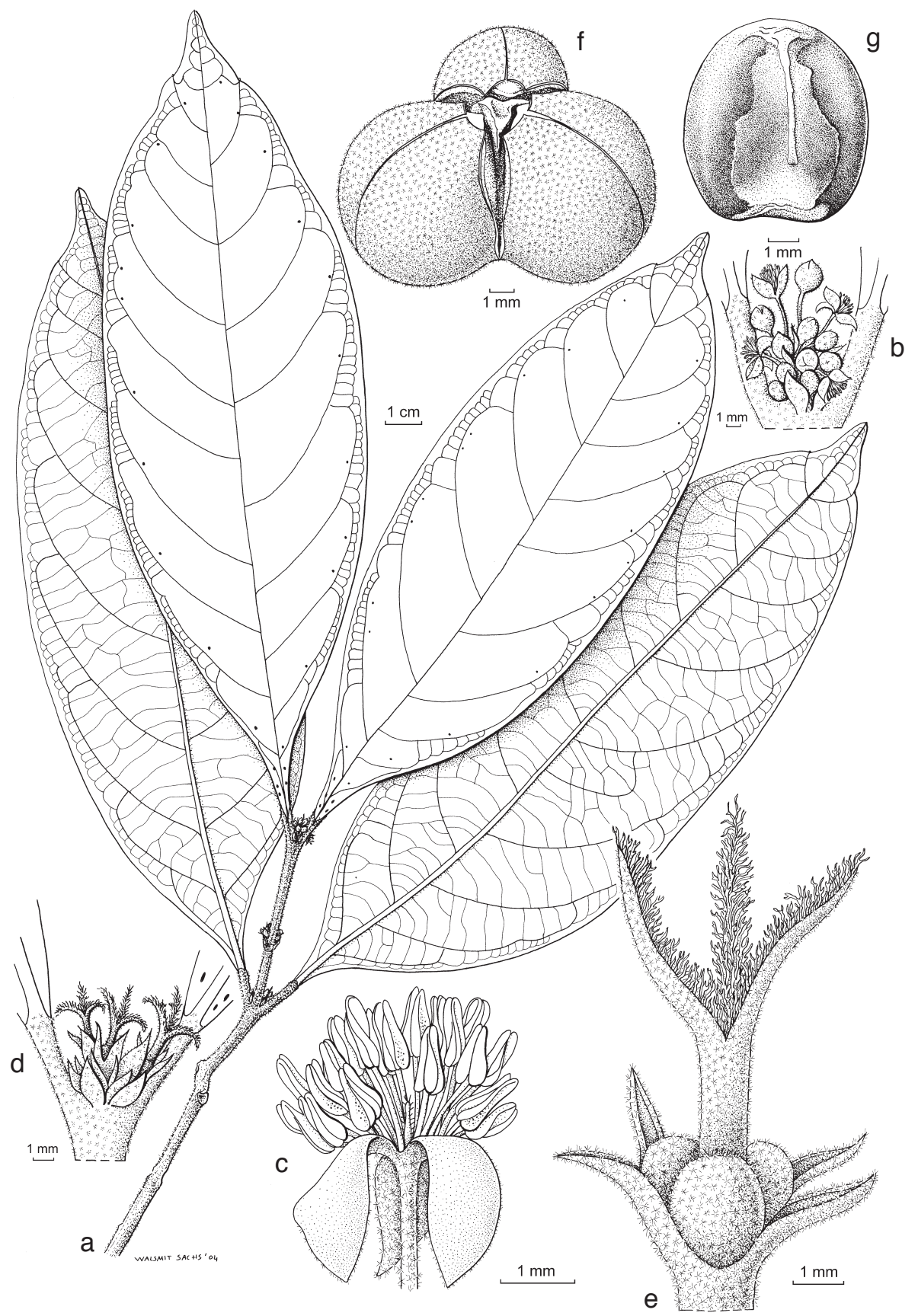

Fig. 5. Mallotus glomerulatus Welzen. a. Habit with staminate inflorescences in bud; b. staminate inflorescence; c. staminate flower; d. pistillate inflorescence; e. pistillate flower; f. fruit; g. seed (a-c: Koonthunthod et al. 524; d, e: Koonthunthod et al. 517; f, g: Pooma et al. 2636; all L). 
rarely present, inconspicuous, marginal in lower half, 1 or 2 per side, $4-5 \mathrm{~mm}$ from margin, orbicular, c. 0.1 by $0.1 \mathrm{~mm}$, lower surface hairy on midrib and nerves, scatteredly covered with glandular hairs, domatia absent, venation pinnate, nerves 8 or 9 per side, looping and closed near margin. Inflorescences racemes, 1-3 together, erect; axes sparsely hairy; peduncle $5-6$ by c. $0.5 \mathrm{~mm}$; bracts triangular to narrowly triangular, $1-2$ by $0.7-1 \mathrm{~mm}$, persistent, margin entire, apex caudate, sparsely hairy; bracteoles absent. Flowers: pedicels sparsely hairy; sepals free, persistent, margin entire, apex acute, sparsely hairy and with glandular hairs outside, glabrous inside. Staminate inflorescences $2.5-5 \mathrm{~cm}$ long; nodes per branch 8-14, with 3-5 flowers per bract. Staminate flowers 3-3.3 mm diam.; pedicels 5-11 mm long; sepals 3, ovate, 2-2.5 by 1.7-2 mm; stamens 60-70, glabrous, filaments $0.5-1.2 \mathrm{~mm}$ long, free, thecae ovoid, $0.2-0.3$ by $0.2-0.25 \mathrm{~mm}$, connective widened; pistillode absent. Pistillate inflorescences 3-3.5 cm long; nodes per branch 1-3, with 1 flower per bract. Pistillate flowers 4-5 mm diam.; pedicels c. $1 \mathrm{~mm}$ long; sepals 3 or 4, triangular, $4.5-5$ by $1.2-1.4$ $\mathrm{mm}$; ovary c. 2 by $2.2 \mathrm{~mm}$, 3-locular, densely hairy, sparsely covered with glandular hairs; style c. $1 \mathrm{~mm}$ long; stigmas c. 7 by $1 \mathrm{~mm}$. Fruits capsules, c. 8 by $13-17 \mathrm{~mm}$, opening septicidally-loculicidally, surface spiny, densely hairy, sparsely covered with glandular hairs; spines c. 1000 , straight, $2.5-3$ by $0.1-0.2 \mathrm{~mm}$, densely hairy; wall c. $1 \mathrm{~mm}$ thick, glabrous inside; column $4.5-5$ by c. $4 \mathrm{~mm}$. Seeds \pm globose, c. 6 by 5.5 by $4 \mathrm{~mm}$, surface smooth, dull, brown.

Distribution - Myanmar, Thailand.

Habitat \& Ecology - Disturbed places in primary, evergreen, seasonal and hardwood forest; in shaded understorey; on granite or shale bedrock. Altitude 475-1100 m. Flowering: October; fruiting: March.

\section{Mallotus hymenophyllus Airy Shaw - Map 4}

Mallotus hymenophyllus Airy Shaw (1968) 381; (1972) 303; Welzen et al. (2000) 100. - Type: Kerr 12389 (holo K; iso BK, L), Thailand, Peninsular, Surat Thani, Sawng Pi Nawng [Song Phi Nong].

Shrubs to small trees, up to $4 \mathrm{~m}$ high, dioecious or monoecious. Outer bark smooth and finely striate. Indumentum composed of simple and tufted hairs and orange glandular hairs. Stipules triangular to narrowly triangular, $1.2-4$ by $0.5-0.7 \mathrm{~mm}$, early caducous, margin entire, apex acute, scatteredly hairy outside, glabrous inside. Leaves opposite; petiole $28-170$ by $0.8-2 \mathrm{~mm}$, sparsely hairy; blade broadly ovate to ovate, $4.8-31$ by $5-24 \mathrm{~cm}$, length/width ratio 1.2-1.5, membranaceous, base cordate, margin denticulate to crenulate, with glandular teeth (located in a minute notch), apex acute to acuminate, upper surface densely to sparsely hairy on midrib and nerves, extrafloral nectaries marginal from base to apex, 2-8 per side, 1-9 $\mathrm{mm}$ from margin, elliptic to orbicular, $0.7-1.2$ by $0.5-0.8 \mathrm{~mm}$, lower surface sparsely to scatteredly hairy on midrib and nerves, scatteredly covered with glandular hairs (sometimes only present near the leaf margin), domatia present, venation triplinerved, nerves 5-7 per side, ending in margin. Inflorescences racemes, 1-3 together, pendulous, unisexual or bisexual; peduncle $28-95$ by $0.5-1.5 \mathrm{~mm}$; axes scatteredly hairy; bracts triangular, $0.9-2$ by 1-2 mm, persistent to caducous, margin entire, apex acute, glabrous; bracteoles absent. Flowers: pedicels sparsely to scatteredly hairy; sepals free, persistent, margin entire, 
apex acute, glabrous. Staminate inflorescences $6-13.7 \mathrm{~cm}$ long, nodes per branch 6-10, with 3-12 flowers per bract. Staminate flowers 3-5 mm diam.; pedicels 3-12 $\mathrm{mm}$ long; sepals 3 or 4 , ovate to elliptic, $2-3.3$ by $1.2-2.7 \mathrm{~mm}$; stamens $28-58$, glabrous, filaments $1-1.5 \mathrm{~mm}$ long, free, thecae ellipsoid, $0.3-0.5$ by $0.2-0.25 \mathrm{~mm}$, connective not widened; pistillode absent. Pistillate inflorescences $8-16.1 \mathrm{~cm}$ long, nodes per branch 5-10, with 1 flower per bract. Pistillate flowers 3-5 mm diam.; pedicels 3-6 $\mathrm{mm}$ long; sepals 4 or 5 , narrowly to linear-triangular, $2.2-3$ by $0.7-0.9 \mathrm{~mm}$; ovary $1.3-1.6$ by $1.5-1.8 \mathrm{~mm}, 3$-locular, glabrous, scatteredly covered with glandular hairs; style $0.8-1.7 \mathrm{~mm}$ long; stigmas $1.5-6$ by $0.6-0.8 \mathrm{~mm}$. Fruits capsules, $6-11$ by $7-14$ $\mathrm{mm}$, dark brown, opening septicidally-loculicidally, surface spiny, scatteredly hairy to glabrous, scatteredly covered with glandular hairs; spines 70-120, curved, $0.5-2$ by $0.2-0.3 \mathrm{~mm}$, hairy at apex; wall $0.8-1 \mathrm{~mm}$ thick, sparsely hairy inside; column $3-5.5$ by $5.2-7 \mathrm{~mm}$. Seeds \pm globose, $4.9-6.2$ by $4.2-6$ by $4.1-6.5 \mathrm{~mm}$, surface smooth, shiny, light cream.

Distribution - Endemic to Thailand.

Habitat \& Ecology - Locally in evergreen and bamboo forest; by streams and in shaded, overgrown places; on limestone. Altitude 20-100 m. Flowering and fruiting throughout the year.

\section{Mallotus khasianus Hook.f. - Map 12}

Mallotus khasianus Hook.f. (1887) 438; Pax \& K. Hoffm. (1914) 191; Airy Shaw (1968) 396; Welzen et al. (2000) 100; W.J. Kress et al. (2003) 232. — Lectotype (selected here): Griffith s.n. (holo K; iso L), India, Meghalaya, Khasi Hills.

Mallotus filiformis Hook.f. (1887) 435. - Lectotype (selected here): Helfer KD 4732 (holo K), Myanmar, Tenasserim.

Mallotus polyneurus Hook.f. (1887) 439. - Type: Helfer KD 4764 (holo K), Myanmar, Tenasserim.

Mallotus esquirolii H. Lév. (1911) 327; (1914) 165; Lauener (1983) 482. - Lectotype (selected here): Esquirol 898 female (holo E; A photo), China, Guizhou.

Shrubs to small trees, 4-14 m high, dioecious or rarely monoecious. Outer bark smooth and finely striate. Indumentum composed of simple and tufted hairs and orange to yellow glandular hairs. Stipules narrowly to linear-triangular, $2.3-3.5$ by $0.2-0.3$ $\mathrm{mm}$, early caducous, margin entire, apex acute, densely hairy outside, glabrous inside. Leaves opposite; petiole $3-75$ by $0.9-1.5 \mathrm{~mm}$, densely to sparsely hairy; blade elliptic to obovate, $6.5-31$ by $2.8-13 \mathrm{~cm}$, length/width ratio $1.8-4.3$, chartaceous, base shallowly emarginate, obtuse to cuneate, margin denticulate to crenulate, with glandular teeth, apex caudate, upper surface scatteredly hairy on midrib and nerves, extrafloral nectaries marginal from base to apex, 1-12 per side, $0-4 \mathrm{~mm}$ from margin, elliptic to orbicular, $0.3-1.7$ by $0.3-1.1 \mathrm{~mm}$, lower surface scatteredly hairy on midrib and nerves, scatteredly covered with glandular hairs, domatia sometimes present, venation pinnate, nerves 7-12 per side, looping and closed near margin. Inflorescences racemes, single (or 2 together), pendulous, unisexual (or bisexual); axes sparsely to densely hairy; peduncle $0.5-80$ by $1-1.8 \mathrm{~mm}$, base with numerous bracts; bracts triangular, $1-3.5$ by $1-2.2 \mathrm{~mm}$, persistent to caducous, margin subentire, apex acute, scatteredly hairy outside, glabrous inside; bracteoles sometimes present, triangular, $1-1.5$ by $0.5-0.7 \mathrm{~mm}$, persistent to caducous. Flowers: pedicels densely to sparsely hairy; sepals 


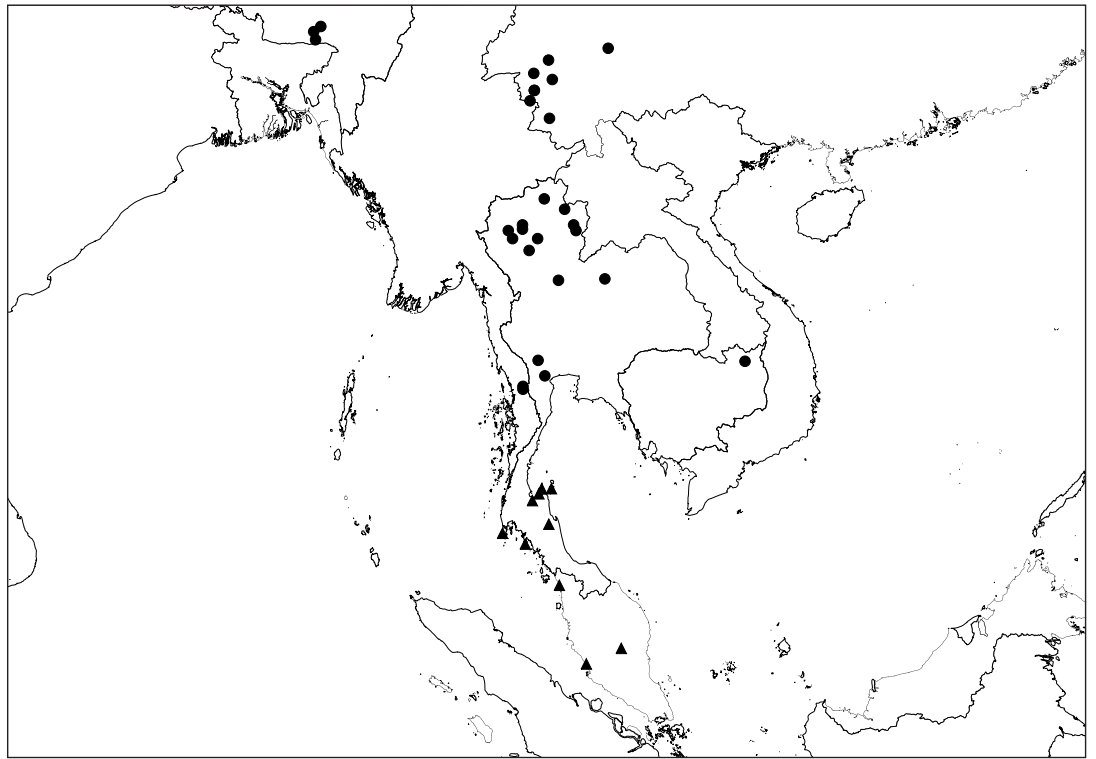

Map 12. Distribution of Mallotus khasianus Hook.f. (•), M. subcuneatus (Gage) Airy Shaw (\).

persistent, margin entire, apex acute, outside sparsely hairy and sometimes sparsely covered with glandular hairs, glabrous insides. Staminate inflorescences $7-53 \mathrm{~cm}$ long, nodes per branch 7-60, with 3-8 flowers per bract. Staminate flowers 5-8 mm diam.; pedicels $5-15 \mathrm{~mm}$ long; sepals 3 or 4 , elliptic, $3-4$ by $2-3 \mathrm{~mm}$, free; disc-glands present; stamens 40-86, filaments $1-4.5 \mathrm{~mm}$ long, free, glabrous, thecae ovoid to ellipsoid, $0.5-0.7$ by $0.25-0.35 \mathrm{~mm}$, sometimes hairy, connective widened; pistillode absent. Pistillate inflorescences 20-45 cm long, nodes per branch (rarely 1-)5-55, with 1 flower per bract. Pistillate flowers 4-8 mm diam.; pedicels 2-5 mm long; calyx 4- or 5-lobed, lobes narrowly triangular, $1.2-3.8$ by $0.2-2 \mathrm{~mm}$; staminodes rarely present; ovary $1.2-2$ by $1.2-5 \mathrm{~mm}, 3$-locular, light yellow to green, densely hairy, scatteredly covered with glandular hairs; style $0.5-2 \mathrm{~mm}$ long; stigmas $5-14$ by $1.6-1.7 \mathrm{~mm}$. Fruits capsules, 9-18 by 10-20 mm, pale green, opening septicidally-loculicidally, surface spiny, sparsely hairy, scatteredly covered with glandular hairs; spines 70-250, curved, $1.2-2.3$ by $0.2-0.3 \mathrm{~mm}$, sparsely hairy; wall $1-1.4 \mathrm{~mm}$ thick, sparsely hairy inside; column $5.5-10.8$ by $6.8-8.2 \mathrm{~mm}$. Seeds \pm globose, $6.8-11$ by $6.9-10.2$ by 6.3-9.2 mm, surface smooth, shiny, dark brown.

Distribution - From E India, Myanmar (Tenasserim) to China (Yunnan, Guizhou, Guangxi), Thailand, Cambodia.

Habitat \& Ecology - Locally common in deciduous and hill evergreen, seasonal forest, open or shaded understorey; along roads and streams, margins of swamp forest or on steep slopes; on granite and limestone bedrock. Altitude 140-2600 m. Flowering and fruiting throughout the year.

Vernacular names - Ngao-pa (Thai).

Uses - The wood is excellent firewood. 


\section{Mallotus korthalsii Müll.Arg. - Map 13}

Mallotus korthalsii Müll.Arg. in DC. (1866) 976; Merr. (1923) 433; Airy Shaw (1975) 170 . - Rottlera korthalsii (Müll.Arg.) Scheff. (1869) 124. - Type: Korthals s.n. (L barcode L0293734), Borneo, Kalimantan Selatan, Gunung Sakumbang.

Mallotus petanodon Airy Shaw (1978a) 401. - Type: Whitmore 3071 (holo K), SE Indonesia, Borneo, Kalimantan Timur (see note 1).

Shrubs to large trees, up to $25 \mathrm{~m}$ high, dbh 4-69 cm, dioecious. Outer bark smooth. Indumentum composed of simple and tufted hairs and orange to dark red glandular hairs. Stipules triangular, $1.5-3$ by $0.8-1 \mathrm{~mm}$, early caducous, margin entire, apex acute to acuminate, densely to scatteredly hairy outside, scatteredly hairy inside. Leaves opposite; petiole $10-90$ by $0.5-1.5 \mathrm{~mm}$, densely to sparsely hairy; blade ovate to obovate, $5.5-25$ by $3-15 \mathrm{~cm}$, length/width ratio $1.2-1.7$, membranaceous to chartaceous, base rounded, obtuse to cuneate, margin entire to dentate, with glandular teeth, apex caudate, upper surface scatteredly hairy on midrib and nerves, extrafloral nectaries marginal from base (to apex), 3-10 per side, $0.5-8 \mathrm{~mm}$ from margin, elliptic to orbicular, $0.3-0.8$ by $0.3-0.8 \mathrm{~mm}$, lower surface scatteredly hairy, densely covered with glandular hairs, domatia sometimes present, venation triplinerved, nerves 3-5 per side (see note 2), looping and closed near margin or ending in margin. Inflorescences racemes, single, erect; axes densely hairy; peduncle 5-12 mm long; bracts deltoid, c. 1 by $0.8-1 \mathrm{~mm}$, persistent to caducous, margin entire, apex acute, densely hairy outside, inside glabrous; bracteoles absent. Flowers: pedicels densely hairy; sepals persistent, margin entire, outside sparsely to scatteredly hairy with simple and glandular hairs, glabrous inside. Staminate inflorescences 3-22 cm long, nodes per branch 20-25, with 3-15 flowers per bract. Staminate flowers $2-5 \mathrm{~mm}$ diam.; pedicels $0.5-3 \mathrm{~mm}$ long; sepals 4 or 5 , ovate to elliptic, $1.8-2.5$ by $0.5-1.5 \mathrm{~mm}$, free; stamens $23-33$,

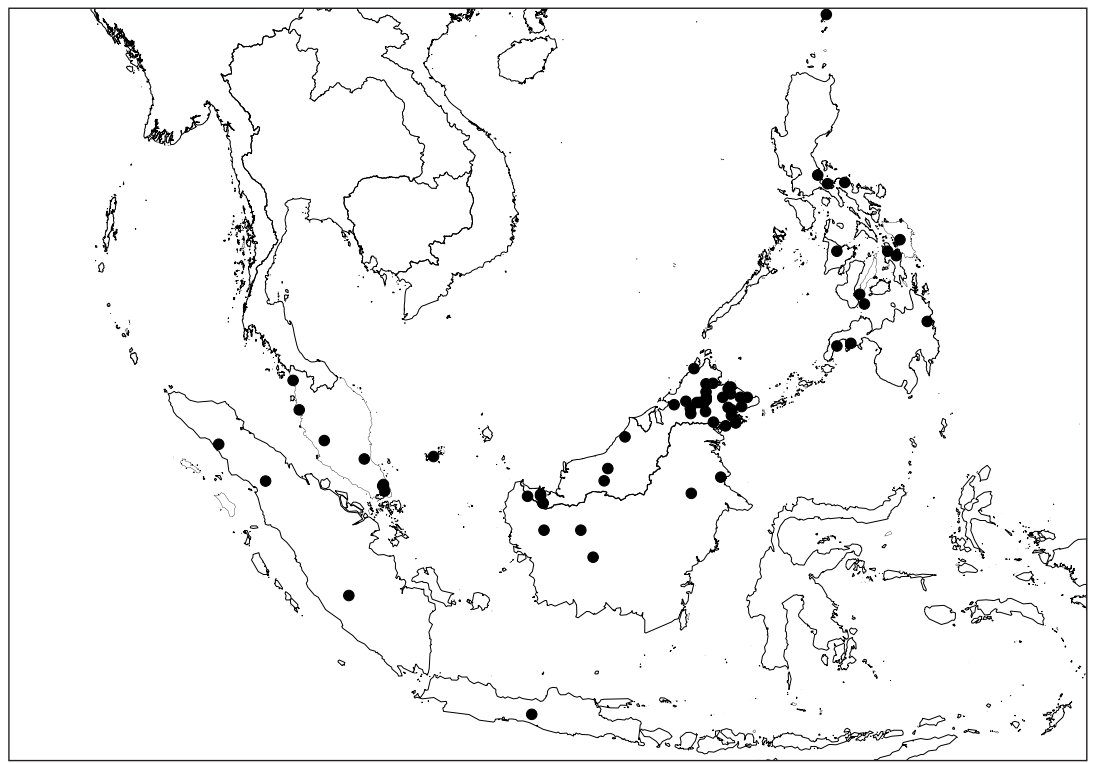

Map 13. Distribution of Mallotus korthalsii Müll.Arg. 
filaments $2.3-3 \mathrm{~mm}$ long, free, hairy to glabrous, thecae ovoid, $0.1-0.2$ by $0.2-0.3$ $\mathrm{mm}$, hairy, connective not widened; pistillode absent. Pistillate inflorescences 4-24 $\mathrm{cm}$ long, nodes per branch 15-80, with 1 flower per bract. Pistillate flowers 3-3.5 mm diam.; pedicels $1.5-3 \mathrm{~mm}$ long; calyx 4-6-lobed, lobes triangular to narrowly triangular, $1.5-3$ by $0.7-1.2 \mathrm{~mm}$, margin subentire; ovary $1.5-2.1$ by $1.9-2.1 \mathrm{~mm}$, 3-locular, densely hairy, scatteredly covered with glandular hairs; style $0.2-0.5 \mathrm{~mm}$ long; stigmas $2-3$ by $1.5-2 \mathrm{~mm}$. Fruits capsules, $5-11$ by $10-14 \mathrm{~mm}$, green, opening septicidally-loculicidally, surface spiny, densely to sparsely hairy, scatteredly covered with glandular hairs; spines (20-) $100-200, \pm$ straight, $1-1.7$ by $(0.1-) 0.3-0.8 \mathrm{~mm}$, hairy; wall 1-1.5 mm thick, densely hairy to glabrous inside; column $5-6$ by c. $4 \mathrm{~mm}$. Seed \pm globose, $3-5$ by $4-5$ by $4-5 \mathrm{~mm}$, surface smooth, shiny, dark brown

Distribution - Peninsular Malaysia, Sumatra, Java, Borneo, Philippines.

Habitat \& Ecology - Primary and secondary dipterocarp forest, marshy and savannah areas; on ridges, along forest edges, road and river sides, on steep slopes and in logged areas; on limestone. Altitude: sea level up to $400 \mathrm{~m}$. Flowering: April to September; fruiting: March to November.

Vernacular name - Sumatra: Kayu jobar.

Notes - 1. Airy Shaw (1978a) reported M.petanodon to be different from M. korthalsii because of the presence of "long spreading repand teeth in the upper half of the leaves, and the almost smooth cocci of the capsules". However, these features are also present in $M$. korthalsii over its entire distribution and are part of the variation of the species. Furthermore, Airy Shaw had only seen one sample from Kalimantan Timur, Borneo (Whitmore 3071) and based on this single collection he described M. petanodon. Until now, we have seen three collections from Kalimantan Timur (Kato \& Wiriadinata B5524; Keßler \& Arbainsyah B1361 and Wiriadinata 811) and they all have the typical characters of $M$. korthalsii. Therefore, M. petanodon is synonymized with M. korthalsii.

2. The first pair of nerves end in the upper half of the leaf blade.

\section{Mallotus lanceolatus (Gagnep.) Airy Shaw - Map 9}

Mallotus lanceolatus (Gagnep.) Airy Shaw (1968) 381; (1972) 303. - Coelodiscus lanceolatus Gagnep. (1923) 50. - Lectotype (selected here): Pierre s.n. (P), Vietnam, Caï-cong.

Mallotus hainanensis S.M. Hwang (1985) 293. - Type: L. Deng 2659 (holo, IBSC, picture seen), China, Hainan, Lingshui.

Shrubs to small trees, up to $8 \mathrm{~m}$ high, dioecious. Indumentum composed of simple and tufted hairs and yellow to orange glandular hairs. Stipules triangular to narrowly triangular, $3-4$ by $0.7-0.9 \mathrm{~mm}$, persistent to caducous, margin entire, apex cuspidate to caudate, sparsely hairy outside, scatteredly hairy inside. Leaves opposite; petiole $2.5-24$ by $0.6-0.8 \mathrm{~mm}$, sparsely to scatteredly hairy; blade ovate to obovate (smaller of each pair ovate to elliptic), 5-12.5(-16) by $2-6.5(-7.5) \mathrm{cm}$, length/width ratio $2-3$, chartaceous (to membranaceous, see note 1), base obtuse to cuneate, margin entire to crenate to (shallowly) dentate, with glandular teeth, apex acute to acuminate, upper surface sparsely hairy on midrib and nerves, sometimes scatteredly covered with glandular hairs, extrafloral nectaries marginal in the lower half, 3-7 per side, $0.2-1 \mathrm{~mm}$ from margin, orbicular, c. 0.2 by $0.2 \mathrm{~mm}$, lower surface scatteredly hairy, glabrescent, sparsely 
covered with glandular hairs, domatia usually present, venation triplinerved, nerves 5-9 per side, looping and closed near margin. Inflorescences racemes, single, erect; peduncle $1.5-4.2$ by c. $0.5 \mathrm{~mm}$; axes sparsely hairy; bracts triangular, c. 3 by $0.5 \mathrm{~mm}$, persistent, margin entire to subentire, apex acuminate, sparsely hairy outside, glabrous inside; bracteoles sometimes present, narrowly triangular, $0.8-1$ by $0.2-0.3 \mathrm{~mm}$, early caducous. Flowers: pedicels sparsely hairy; sepals 3 or 4, c. 3 by $4 \mathrm{~mm}$, free, persistent, margin entire, apex acute, sparsely hairy outside, glabrous inside, with glandular hairs outside. Staminate inflorescences $1.2-9 \mathrm{~cm}$ long, nodes per branch 10-15, with 1-7 flowers per bract. Staminate flowers 3-3.6 mm diam.; pedicels 1.5-2.5 mm long; sepals elliptic to obovate, c. 2.2 by $1.2 \mathrm{~mm}$; stamens $30-60$, filaments $0.5-2 \mathrm{~mm}$ long, free, glabrous, thecae ovoid, $0.2-0.3$ by $0.2-0.25 \mathrm{~mm}$, sometimes hairy, connnective widened; pistillode present. Pistillate inflorescences $4-8 \mathrm{~cm}$ long, nodes per branch c. 14, with 1 flower per bract. Pistillate flowers $1.8-3 \mathrm{~mm}$ diam.; pedicels $0.8-2.5 \mathrm{~mm}$ long; sepals narrowly triangular, $2-2.2$ by $0.5-0.7 \mathrm{~mm}$; ovary $1.2-1.5$ by $1.2-1.4 \mathrm{~mm}$, 3-locular, sparsely hairy, sparsely covered with glandular hairs; style $0.4-0.7 \mathrm{~mm}$ long; stigmas $1.2-2.5$ by $0.8-1.2 \mathrm{~mm}$. Fruits capsules, $3-6$ by $6-8.8 \mathrm{~mm}$, green, opening septicidally-loculicidally, surface spiny, sparsely hairy, sparsely covered with glandular hairs; spines $70-120, \pm$ straight, $1-3$ by $0.1-0.2 \mathrm{~mm}$; wall $0.5-0.7 \mathrm{~mm}$ thick, densely to sparsely hairy inside; column $2.2-3.3$ by $2.2-3 \mathrm{~mm}$. Seeds \pm globose, $2.5-3$ by $2-3$ by $2.2-2.5 \mathrm{~mm}$, surface smooth, shiny, dark brown.

Distribution - Thailand, Cambodia, Laos, Vietnam, S China (Guangxi, Guizhou, Hainan, Yunnan).

Habitat \& Ecology - Secondary, mixed, seasonal evergreen or deciduous hardwood forest; by streams; on limestone, calcareous rock, sandy or shale bedrock soil. Altitude 60-1300 m. Flowering and fruiting: February to December.

Notes - 1. Shimizu et al.T-27084,T-27085 differ from the other specimens studied by the presence of membranaceous leaves.

2. See also the notes for M. minimifructus.

\section{Mallotus lauterbachianus (Pax \& K. Hoffm.) Pax \& K. Hoffm. - Map 7}

Mallotus lauterbachianus (Pax \& K. Hoffm.) Pax \& K. Hoffm. (1914) 157. - Coelodiscus lauterbachianus Pax \& K. Hoffm. in Pax (1910) 481. - Type: Weinland 257 (ULM? n.v.), Papua New Guinea, Bumi.

Mallotus lullulae Airy Shaw (1968) 384. - Type: Brass 28701 (holo K; iso L, S), Papua New Guinea, Woodlark Island, Kulumadau.

Shrubs to small trees, 2-9 m high; branches hollow, dioecious. Indumentum composed of simple and stellately-tufted hairs, glandular hairs absent. Stipules narrowly triangular, c. 4.5 by $1 \mathrm{~mm}$, early caducous, margin entire, apex acute, sparsely hairy outside, glabrous inside. Leaves opposite (to subopposite); petiole 10-103 by $1-2 \mathrm{~mm}$, scatteredly hairy; blade ovate to elliptic, $10.5-30$ by $6.5-14 \mathrm{~cm}$, length/width ratio 1.6-2.1, chartaceous, base rounded, obtuse to cuneate, margin crenulate, with glandular teeth, apex cuspidate, upper surface glabrous, extrafloral nectaries along the midrib, $5-18$ per side, elliptic to orbicular, $0.8-1$ by $0.7-1 \mathrm{~mm}$, lower surface scatteredly hairy, glabrescent, domatia present, venation triplinerved, nerves 5-9 per side, indistinctly looping and closed near margin. Inflorescences single, erect; axes sparsely hairy; bracts triangular to narrowly triangular, $2.5-5$ by $0.8-1.8 \mathrm{~mm}$, persistent, margin entire, apex 
acute, sparsely hairy outside, glabrous inside; bracteoles narrowly triangular, 1-3 by 0.3-1 mm, persistent. Flowers: pedicels densely hairy; sepals free, margin entire, apex acute, persistent, densely to sparsely hairy outside, scatteredly hairy to glabrous inside. Staminate inflorescences racemes, 5-14 cm long; peduncle 5-60 mm long; nodes per branch 5-8, with 8-10 flowers per bract. Staminate flowers 5-8 mm diam.; pedicels $6-10 \mathrm{~mm}$ long; sepals 3 , ovate to elliptic, $4-6$ by $2-3 \mathrm{~mm}$; stamens $30-65$, filaments $1.8-5 \mathrm{~mm}$ long, free, hairy, thecae ovoid, $0.3-0.4$ by $0.2-0.25 \mathrm{~mm}$, glabrous, connective widened; pistillode present. Pistillate inflorescences racemes or umbel-like, 2.7-4 cm long; peduncle 20-27 mm long; nodes per branch 1-4, with 1-3 flowers per bract. Pistillate flowers 3-4 mm diam.; pedicels 4-6 mm long; sepals 3, narrowly to linear-triangular, $6-7.1$ by $0.8-1 \mathrm{~mm}$; ovary $2.5-2.8$ by $2-2.3 \mathrm{~mm}, 3$-locular, densely hairy; style c. 2 mm long; stigmas $3-4.5$ by $0.8-1 \mathrm{~mm}$. Fruits unknown.

Distribution - Endemic to New Guinea.

Habitat \& Ecology - Frequent in secondary forest. Altitude 10-500 m. Flowering and fruiting: July to November.

\section{Mallotus leucocalyx Müll.Arg. - Map 5; Plate 1a-c}

Mallotus leucocalyx Müll.Arg. in DC. (1866) 970. - Type: Unit. Stat. Explor. Exped. s.n. (holo G-DC, IDC microfiche 800; iso GH, US), Philippines, Mindanao, Caldera.

Shrubs to small trees, up to $8 \mathrm{~m}$ high, dioecious. Outer bark smooth. Indumentum composed of simple, tufted and stellately-tufted hairs and yellow to orange glandular hairs. Stipules triangular to narrowly triangular, $4-10$ by $2-4 \mathrm{~mm}$, persistent to caducous, margin entire to subentire, apex cuspidate, scatteredly hairy to glabrous outside, densely hairy inside. Leaves opposite; petiole $20-100$ by $1.3-1.8 \mathrm{~mm}$, densely to sparsely hairy; blade ovate to obovate, $10.5-26$ by $5.8-15 \mathrm{~cm}$, length/width ratio $1.5-1.9$, membranaceous, base shallowly emarginate, rounded to cuneate, margin denticulate to dentate, with glandular teeth, apex acute to acuminate, upper surface scatteredly hairy to glabrous on midrib, extrafloral nectaries marginal throughout the blade, up to 12 per side, 1-7 mm from margin, sometimes also all over the blade, elliptic to orbicular, $0.3-0.5$ by $0.3-0.5 \mathrm{~mm}$, lower surface sparsely hairy, sparsely covered with glandular hairs, domatia absent, venation triplinerved, nerves 8 or 9 per side, looping and closed near margin or ending in margin. Inflorescences racemes, single, erect; peduncle 15-28 by c. $1 \mathrm{~mm}$; axes densely hairy; bracts trilobed, persistent, margin entire, apex acuminate to cuspidate, densely hairy outside, glabrous inside; bracteoles absent. Flowers: pedicels densely hairy; sepals free, persistent, margin entire, outside densely to sparsely hairy and scatteredly covered with glandular hairs, glabrous inside. Staminate inflorescences $3.5-12 \mathrm{~cm}$ long, nodes per branch $8-23$, with $2-7$ flowers per bract; bracts $5-8$ by 3-4 mm. Staminate flowers 3-4.2 mm diam.; pedicels 2-4 mm long; sepals 3 or 4, elliptic to obovate, $2.5-2.8$ by $0.7-1.2 \mathrm{~mm}$, apex acuminate; stamens $20-40$, glabrous, filaments $1-3 \mathrm{~mm}$ long, free, pale light yellow, thecae ovoid, $0.2-0.3$ by $0.2-0.25 \mathrm{~mm}$, connective widened; pistillode present. Pistillate inflorescences $9.4-16 \mathrm{~cm}$ long; nodes per branch 7-15, with 1 flower per bract; bracts $5-10$ by $3-5 \mathrm{~mm}$. Pistillate flowers 2.8-3 mm diam.; pedicels $1-2 \mathrm{~mm}$ long; sepals 3-6, narrowly triangular, 3.8 -4.1 by 0.8-1 mm, apex acute; ovary $1.8-2$ by $1.8-2 \mathrm{~mm}, 3$-locular, densely hairy, pale light green, scatteredly covered with glandular hairs; style $1.5-2 \mathrm{~mm}$ long; stigmas $4-5$ by $1.3-1.5 \mathrm{~mm}$. Fruits capsules, $7-7.5$ by $9-11 \mathrm{~mm}$, green, opening septicidally-loculi- 
cidally, surface spiny, densely hairy, scatteredly covered with glandular hairs; spines $200-230$, curved, $3-4.2$ by $0.2-0.3 \mathrm{~mm}$, densely hairy; wall $0.7-1 \mathrm{~mm}$ thick, densely to sparsely hairy inside; column $3.4-5$ by $3-3.5 \mathrm{~mm}$. Seeds \pm globose, $3.2-4.2$ by 3-4 by 3-3.8 mm, surface smooth, shiny, light brown.

Distribution - Myanmar, Thailand, Peninsular Malaysia, Sumatra, Java, Borneo (Sabah), Philippines, Sulawesi.

Habitat \& Ecology - In understorey of primary or secondary forests, in open or shaded areas; on clay or limestone. Altitude: sea level up to $650 \mathrm{~m}$. Flowering and fruiting throughout the year.

Vernacular names - Borneo (Sabah): Dulang jantan (Bajau), mandahasi (Dusun kinabatangan), sempurna.

\section{Mallotus longinervis M. Aparicio, spec.nov. - Fig. 6; Map 10}

Folia opposite non valde inaequalia chartacea ad coriacea, apice caudato, trinervia, nectaria marginalia 4-6 et nervi secundarii 2 vel 3 per latere, domatia adsunt vel non. Flores staminales 1.8-2 mm diam., pistillodium deest. Flores pistillati 2-2.5 mm diam. Fructus dense glandulose pubescentes. - Typus: S (Banyeng ak Nudong \& Sibat ak Luang) 26206 (holo L; iso A, BO, K, SAN, SING), Borneo, Sarawak, Kuching, Semengoh Arboretum, 12th mile Penrissen Road.

Small trees, 2-8 m high, dioecious. Indumentum composed of simple and stellatelytufted hairs and yellow to orange glandular hairs. Stipules deltoid, $0.4-0.5$ by $0.4-0.5$ $\mathrm{mm}$, early caducous, margin entire, hairy, apex acute, densely hairy. Leaves opposite; petiole $10-50$ by c. $1 \mathrm{~mm}$, sparsely to scatteredly hairy; blade elliptic, $10-25$ by $3.5-8$ $\mathrm{cm}$, length/width ratio $2-3$, chartaceous to subcoriaceous, base obtuse to cuneate, margin entire to denticulate, with glandular teeth, apex caudate, upper surface glabrous, extrafloral nectaries marginal in lower half, 4-6 per side, 1-5 mm from margin, elliptic to orbicular, $0.5-0.8$ by $0.5-0.8 \mathrm{~mm}$, lower surface scatteredly hairy to glabrous, densely covered with glandular hairs, domatia sometimes present, venation triplinerved, nerves 2 or 3 per side, looping and closed near margin. Inflorescences racemes, single, erect; peduncle $2-9$ by $0.5-1 \mathrm{~mm}$; axes densely to sparsely hairy; bracts deltoid, $0.6-0.9$ by $0.5-0.9 \mathrm{~mm}$, persistent to caducous, margin entire, apex acute, densely hairy; bracteoles absent. Flowers: pedicels densely hairy; sepals free, persistent, margin entire, apex acute, densely hairy, also with glandular hairs outside, scatteredly hairy to glabrous inside. Staminate inflorescences 8-12 cm long, nodes per branch 10-18, with 3-5 flowers per bract. Staminate flowers $1.8-2 \mathrm{~mm}$ diam.; pedicels $0.5-0.8 \mathrm{~mm}$ long; sepals 3 , obovate, $1.8-2.5$ by $1.3-1.5 \mathrm{~mm}$; stamens $18-25$, filaments $0.5-1.5 \mathrm{~mm}$ long, free, glabrous, thecae ellipsoid, $0.2-0.3$ by $0.1-0.2 \mathrm{~mm}$, hairy, connective not widened; pistillode absent. Pistillate inflorescences 6-17 cm long, nodes per branch 6-15, with 1 flower per bract. Pistillate flowers 2-2.5 mm diam.; pedicels $2.5-3 \mathrm{~mm}$ long; sepals 5 or 6 , narrowly triangular, $1.5-2$ by $0.3-0.5 \mathrm{~mm}$; ovary $1-1.3$ by $1-1.2 \mathrm{~mm}, 3$-locular, densely hairy, sparsely covered with glandular hairs; style $0.5-1 \mathrm{~mm}$ long; stigmas $1.8-2.3$ by c. $0.8 \mathrm{~mm}$. Fruits capsules, $5-5.5$ by 5.5-6 mm, green, opening septicidally-loculicidally, surface smooth, densely hairy, sparsely covered with glandular hairs; wall $0.1-0.2 \mathrm{~mm}$ thick, densely hairy inside; column $2-2.2$ by $1.5-2 \mathrm{~mm}$. Seeds globose, $2-2.2$ by $1.7-1.8$ by $2-2.5 \mathrm{~mm}$, surface rugose, dull, brown. 


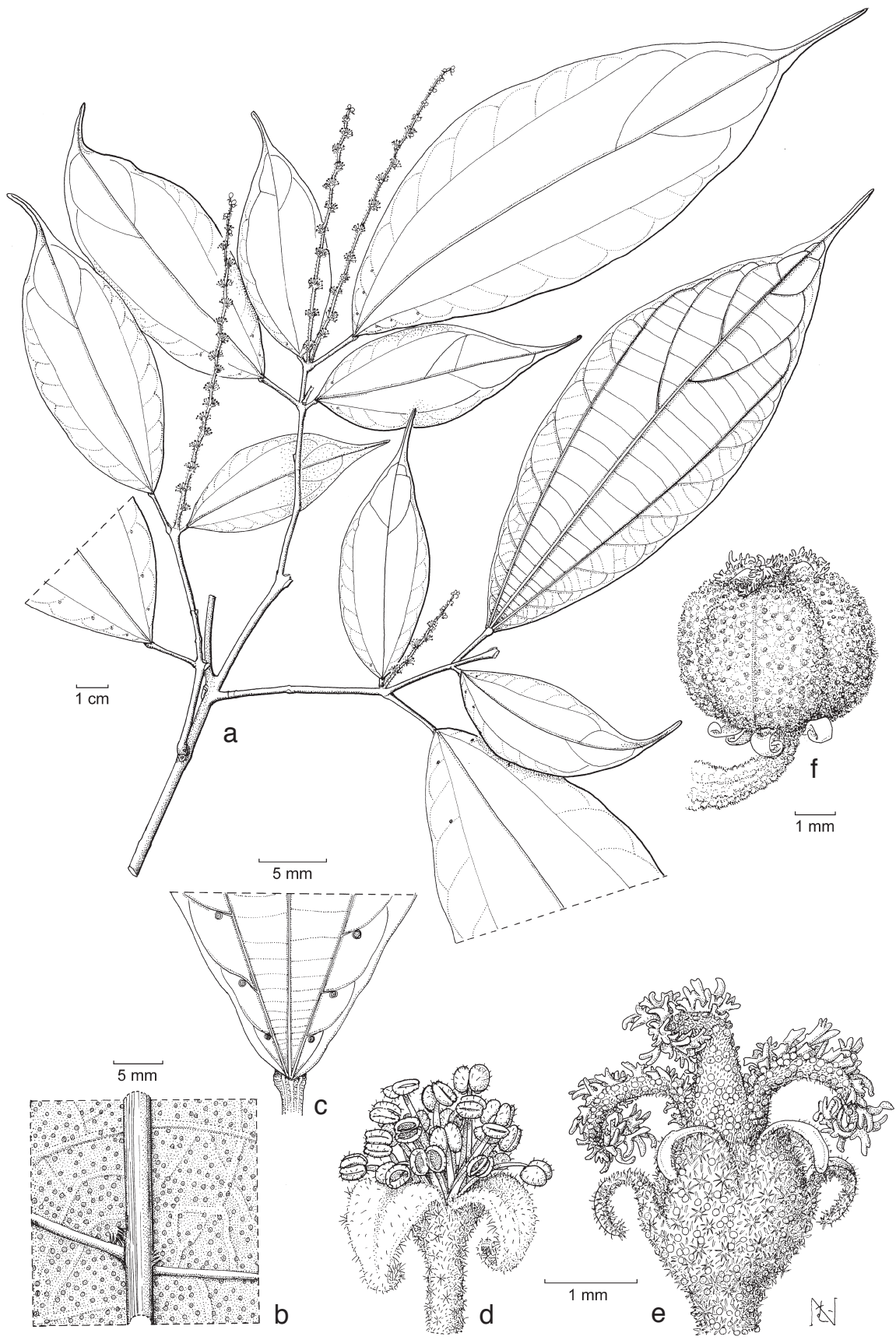

Fig. 6. Mallotus longinervis M. Aparicio. a. Habit with staminate inflorescences; b. detail of lower leaf surface with glandular hairs and domatia; c. base of upper leaf surface with extrafloral nectaries; d. staminate flower; e. pistillate flower; f. fruit (a-d: S (Rosli) 3389; e: S (J.A.R. Anderson) 25990; f. $S$ (Banyeng ak Nudong) 26206; all L). 
Distribution - Endemic to Borneo (Arboretum Semengoh F.R.).

Habitat \& Ecology - Road and river sides. Altitude c. 90 m. Flowering: August; fruiting: November.

Vernacular name - Borneo: Entaempulor (Iban).

Note - The collection $S 3389$ from Sarawak was identified by Airy Shaw as M. smilaciformis (see Dubious species, probably M. korthalsii), but we think that it represents M. longinervis. According to the protologue (syntypes not seen) M. smilaciformis should have 3-5 nerves and minute processes (spines), while $S 3389$ has 2 or 3 nerves and no spines on the fruits. Additionally, M. smilaciformis is reported for Peninsular Malaysia, while $S 3389$ is found in Borneo. So far, all collections seen of M. longinervis were collected in Sarawak in the Semengoh Arboretum, therefore, it is possibly endemic to that place.

\section{Mallotus macularis Airy Shaw - Map 7}

Mallotus macularis Airy Shaw (1971) 526; (1980c) 168. - Type: BW (Schram) 9349 (holo L), New Guinea, Bakaro, Mankowari.

Shrubs, 2-5 m high, dioecious. Indumentum composed of simple and stellately-tufted hairs, glandular hairs absent. Stipules narrowly triangular, $3-7$ by $0.7-1.5 \mathrm{~mm}$, early caducous, margin entire, apex acute, densely hairy outside, sparsely hairy inside. Leaves opposite; petiole $10-84$ by $1.8-2 \mathrm{~mm}$, densely hairy; blade ovate to elliptic, $15-31$ by $5.5-15 \mathrm{~cm}$, length/width ratio $1.7-2.6$, chartaceous, base shallowly emarginate, rounded, obtuse to cuneate, margin entire, undulate, without glandular teeth, apex acute to caudate, upper surface glabrous, extrafloral nectaries along the midrib, sometimes also all over the blade, elliptic to orbicular, $0.6-0.8$ by $0.6-0.8 \mathrm{~mm}$, lower surface scatteredly hairy, domatia present, venation inconspicuously triplinerved, nerves 6-10 per side, indistinctly looping and closed near margin or ending in margin. Inflorescences single, erect; axes densely hairy; bracts linear-triangular, 2.9-3.1 by $0.5-0.7$ $\mathrm{mm}$, persistent, margin entire, apex acute, glabrous; bracteoles triangular $0.9-1.1$ by 0.8-1 mm, caducous, margin entire, apex acute. Flowers: pedicels densely hairy; sepals free, persistent, margin entire, apex acute. Staminate inflorescences racemes, 4.5-6 cm long; peduncle 6-19 mm long; nodes per branch 5-7, with 8-11 flowers per bract. Staminate flowers 5-5.5 mm diam.; pedicels 3-4.5 mm long; sepals 3, ovate to elliptic, $3-5.5$ by $2-2.5 \mathrm{~mm}$; stamens $55-65$, glabrous, filaments $2.5-4 \mathrm{~mm}$ long, free, thecae ovoid, $0.2-0.3$ by $0.2-0.25 \mathrm{~mm}$, connective widened; pistillode present. Pistillate inflorescences umbel-like, 12-17 cm long, nodes per branch 1, with 2 or 3 flowers per bract. Pistillate flowers 3-3.5 mm diam.; pedicels 6-7 mm long; sepals 3 , triangular, $2.9-3.1$ by $1.4-1.6 \mathrm{~mm}$, densely hairy outside, glabrous inside; ovary $2.8-3$ by $2.8 \mathrm{~mm}, 3$-locular, densely hairy, scatteredly covered with glandular hairs; style $0.6-0.8 \mathrm{~mm}$ long; stigmas c. 2 by $0.8 \mathrm{~mm}$. Fruits capsules, $7.5-9$ by $14-17 \mathrm{~mm}$, opening septicidally-loculicidally, surface spiny, densely hairy, scatteredly covered with glandular hairs; spines $70-90$, straight, $1-1.2$ by c. $0.8 \mathrm{~mm}$, densely hairy; wall $1 \mathrm{~mm}$ thick, sparsely hairy inside; column $5.5-7$ by $4-6.8 \mathrm{~mm}$. Seeds \pm globose, 7-7.5 by 6-7 by $6-7 \mathrm{~mm}$, surface smooth, shiny, dark brown.

Distribution - Endemic to New Guinea. 
Habitat \& Ecology — In primary or disturbed lowland forest; on limestone or sandy peaty clay soil. Altitude: sea level to $130 \mathrm{~m}$. Flowering and fruiting throughout the year.

Note - Plants have a strong smell when dried.

\section{Mallotus minimifructus S.E.C. Sierra, spec.nov. - Fig. 7; Map 5}

Folia opposita inaequalia, redacto obcordato nectaria extrafloralia marginalia, nervi 6-9 per latere. Inflorescentia racemosa. Flores staminales: filamentis liberis, discis adsunt. Flores pistillata: stylus $0.2-0.6 \mathrm{~mm}$ longus. Fructus spinosus. - Typus: S (Y.P. Ching) 62305 (holo L; iso CGE, K, KEP, SAN), Borneo, Sarawak, Ulu Sungai Kebhor.

Shrubs to small trees, 3-10 $\mathrm{m}$ high; bole up to $5 \mathrm{~m}$ high, dioecious or rarely monoecious, crown up to $5 \mathrm{~m}$ long. Outer bark smooth. Indumentum composed of simple and tufted hairs and yellow to orange glandular hairs. Stipules triangular to narrowly triangular, 4-10 by $1.4-1.6 \mathrm{~mm}$, persistent to caducous, margin entire, apex acute to caudate, sparsely hairy outside, scatteredly hairy to glabrous inside. Leaves opposite, petiole 1-50 by $0.6-1 \mathrm{~mm}$, densely hairy to scatteredly hairy; blade ovate to obovate (the smaller leaf obcordate), $14-22$ by $5-10 \mathrm{~cm}$, length/width ratio $1.8-3.3$, membranaceous (to chartaceous), base shallowly emarginate, obtuse to cuneate, margin denticulate, dentate to crenate, with glandular teeth, apex caudate, upper surface densely to scatteredly hairy on midrib and nerves, extrafloral nectaries marginal in lower half, $2-7$ per side, 1-2.5 $\mathrm{mm}$ from margin, elliptic to orbicular, $0.2-0.3$ by $0.2-0.3 \mathrm{~mm}$, lower surface sparsely to scatteredly hairy, sparsely covered with glandular hairs, domatia rarely present, venation triplinerved, nerves 6-9 per side, indistinctly looping and closed near margin or ending in margin. Inflorescences racemes (see note 1), single, erect, unisexual or rarely bisexual; axes densely to sparsely hairy; peduncle $9-24$ by $0.8-1 \mathrm{~mm}$; bracts triangular, persistent, margin entire, apex acute, sparsely hairy outside, glabrous inside; bracteoles sometimes present, triangular, $1-1.5$ by $0.2-0.3 \mathrm{~mm}$, early caducous. Flowers: pedicels densely to sparsely hairy; sepals 3 or 4, free, persistent, margin entire, apex acute, densely hairy and with glandular hairs outside, glabrous inside. Staminate inflorescences 6-10 cm long, nodes per branch 12-27, with 1-5 flowers per bract; bracts $1.2-1.4$ by $0.5-0.6 \mathrm{~mm}$. Staminate flowers $3.3-4 \mathrm{~mm}$ diam.; pedicels $1.7-3 \mathrm{~mm}$ long; sepals elliptic to obovate, $2.8-3.2$ by $1-1.2 \mathrm{~mm}$; disc-glands present; stamens 40-90, glabrous, filaments $1-2.5 \mathrm{~mm}$ long, free, thecae ovoid, $0.2-0.3$ by $0.2-0.25$ $\mathrm{mm}$, connective widened; pistillode absent. Pistillate inflorescences $3.4-12 \mathrm{~cm}$ long, nodes per branch $10-20$, with 1 flower per bract; bracts $1.5-1.7$ by $0.6-0.7 \mathrm{~mm}$; bracteoles absent. Pistillate flowers 2.8-3.8 mm diam.; pedicels 1-3 mm long; sepals narrowly triangular, $2-2.4$ by $0.8-1 \mathrm{~mm}$; staminodes rarely present; ovary $1.3-1.5$ by $1.3-1.6 \mathrm{~mm}$, 3-locular, sparsely (to densely, see note 2 ) hairy, sparsely covered with glandular hairs; style $0.2-0.6 \mathrm{~mm}$ long; stigmas $2.2-5.5$ by $0.8-1.2 \mathrm{~mm}$. Fruits capsules, $5-10$ by $8-11 \mathrm{~mm}$, green, opening septicidally-loculicidally, surface spiny, sparsely (to densely) hairy, sparsely covered with glandular hairs; spines 100-200 (rarely more), curved, $1.5-3.5$ by $0.1-0.2 \mathrm{~mm}$, sparsely hairy, red; wall $0.7-0.9 \mathrm{~mm}$ thick, glabrous inside; column $3.8-4$ by $3.5-4 \mathrm{~mm}$. Seed \pm globose, $4-5.1$ by $3.5-5$ by $3.5-4.5 \mathrm{~mm}$, surface smooth, shiny, dark brown.

Distribution - Borneo, Philippines, Sulawesi. 

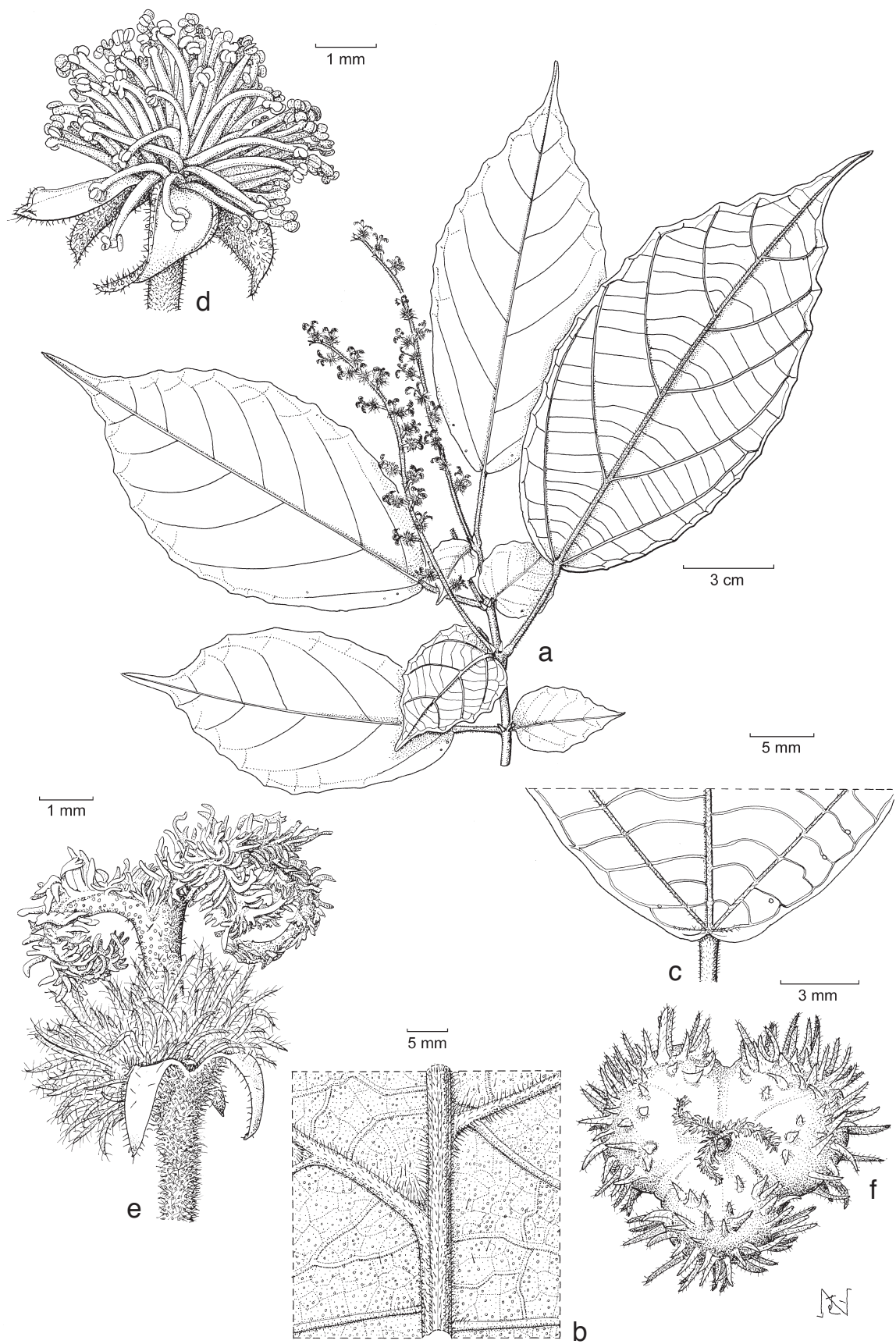

b

Fig. 7. Mallotus minimifructus S.E.C. Sierra. a. Habit with pistillate inflorescence; b. detail of lower leaf surface with glandular hairs; $c$. base of upper leaf surface with extrafloral nectaries; d. staminate flower; e. pistillate flower; f. fruit (a-c: Kostermans 13762; d: W.L.Chew 448; e: J. \& M.S. Clemens 20052; f: Keßler et al. PK1604; a-d, f: L, e: B). 
Habitat \& Ecology - Locally common in understorey of primary forest or in disturbed areas; on basalt, clay-loam, limestone, sandy soil or ultramafic soil. Altitude: sea level up to $800 \mathrm{~m}$. Flowering and fruiting throughout the year.

Vernacular names - Borneo (Sarawak): Entak empuluh. Philippines: Tiolu gletin. Uses - Roots are used against fever.

Notes - 1. Angian 10494 has inflorescences with very short internodes at the apex.

2. SAN 42227 differs from the other material studied in having fruits that are densely covered with spines and hairs resembling those of M. leucocalyx.

3. Mallotus minimifructus resembles M. lanceolatus in leaf appearance, but differs in having larger stipules, $4-10$ by $1.4-1.6 \mathrm{~mm}$ (smaller, $3-4$ by $0.7-0.9 \mathrm{~mm}$ in M. lanceolatus), obcordate reduced leaves (obtuse to elliptic in M. lanceolatus), absence of glandular hairs on upper leaf surface (usually present in M. lanceolatus), leaves drying greenish (usually brownish in M. lanceolatus), domatia rarely present (usually present in M. lanceolatus) and staminate flowers with disc-glands (absent in M. lanceolatus).

4. See also the note for M. dispar.

\title{
27. Mallotus mirus S.E.C. Sierra, spec. nov. - Fig. 8; Map 1
}

\begin{abstract}
Indumentum pilis simplicibus et complanatis caespitosis. Stipulae persistentes. Petioli pilis dispersis. Folia opposita obovata basi cuneata margine undulato supra glabra infra costam versus pubescentia, sine piliis glandulosis. Inflorescentia feminea ut videtur ad flore terminali redacta. Fructus extus pilis dispersis tecti. - Typus: Kerr 19835 (holo L; iso BM, K), Thailand, South-eastern, Prachinburi Circle, Kao Singto.
\end{abstract}

Mallotus spec. nov., Airy Shaw (1968) 396.

Shrubs, up to $0.5 \mathrm{~m}$ high, dioecious. Indumentum composed of simple and stellatelytufted hairs, glandular hairs absent. Stipules triangular to narrowly triangular, $6-6.5$ by 1.5-2 mm, persistent, margin entire, apex acute, scatteredly hairy to glabrous. Leaves opposite; petiole $10-33$ by $1-1.8 \mathrm{~mm}$, sparsely hairy; blade obovate, $14-22$ by $5.5-9$ $\mathrm{cm}$, length/width ratio $2-2.9$, chartaceous, base cuneate, margin dentate to sinuate in upper half, apex caudate, upper surface densely to scatteredly hairy on midrib and nerves, larger extrafloral nectaries marginal in lower half, sometimes along the midrib, 2 or 3 per side, $1-6 \mathrm{~mm}$ from margin, elliptic, $0.7-0.9$ by $0.3-0.5 \mathrm{~mm}$, smaller ones from base to apex $4-8$ per side, $4-5 \mathrm{~mm}$ from margin, orbicular, $0.2-0.3$ by $0.2-0.3$ $\mathrm{mm}$, lower surface scatteredly hairy, domatia absent, venation pinnate, nerves 7-9 per side, ending in margin. Staminate inflorescences and flowers unknown. Infructescences reduced to 1 terminal fruit, axis 1.1-1.7 cm long; single, erect; peduncle absent, sparsely hairy; bracts narrowly triangular, $4.8-5$ by $0.8-1 \mathrm{~mm}$, persistent to caducous, margin entire, apex acute, sparsely hairy outside, glabrescent, glabrous inside; bracteoles absent. Fruits capsules, 3-locular, 10-11 by 11-12 mm, opening septicidally-loculicidally, surface smooth, scatteredly hairy; wall c. $0.8 \mathrm{~mm}$ thick, glabrous inside; column 6-7 by 5-6 mm; calyx 5- or 6-lobed, lobes narrowly triangular, $8-10$ by $1.5-2 \mathrm{~mm}$. Seeds \pm globose, $6-7.8$ by $5-6$ by $5-6 \mathrm{~mm}$, surface smooth, dull, light cream brown.

Distribution - Endemic to Thailand.

Habitat \& Ecology - In evergreen forest, on limestone hills. Altitude $50 \mathrm{~m}$. Fruiting: November. 
Note - Mallotus mirus closely resembles $M$. calocarpus, but differences in morphology and distribution show that they are separate species. Mallotus mirus differs in having scatteredly hairy to glabrous stipules (densely hairy in M.calocarpus), appressed tufted hairs (erect in M. calocarpus), scattered hairs on fruits and petioles (densely hairy in M.calocarpus), cuneate leaf base (attenuate in M.calocarpus). Although both species occur in south-eastern Thailand they are found in different provinces, M. mirus is only known from Kao Singto (Prachin Buri Prov.), while M. calocarpus is endemic to Chanthaburi.

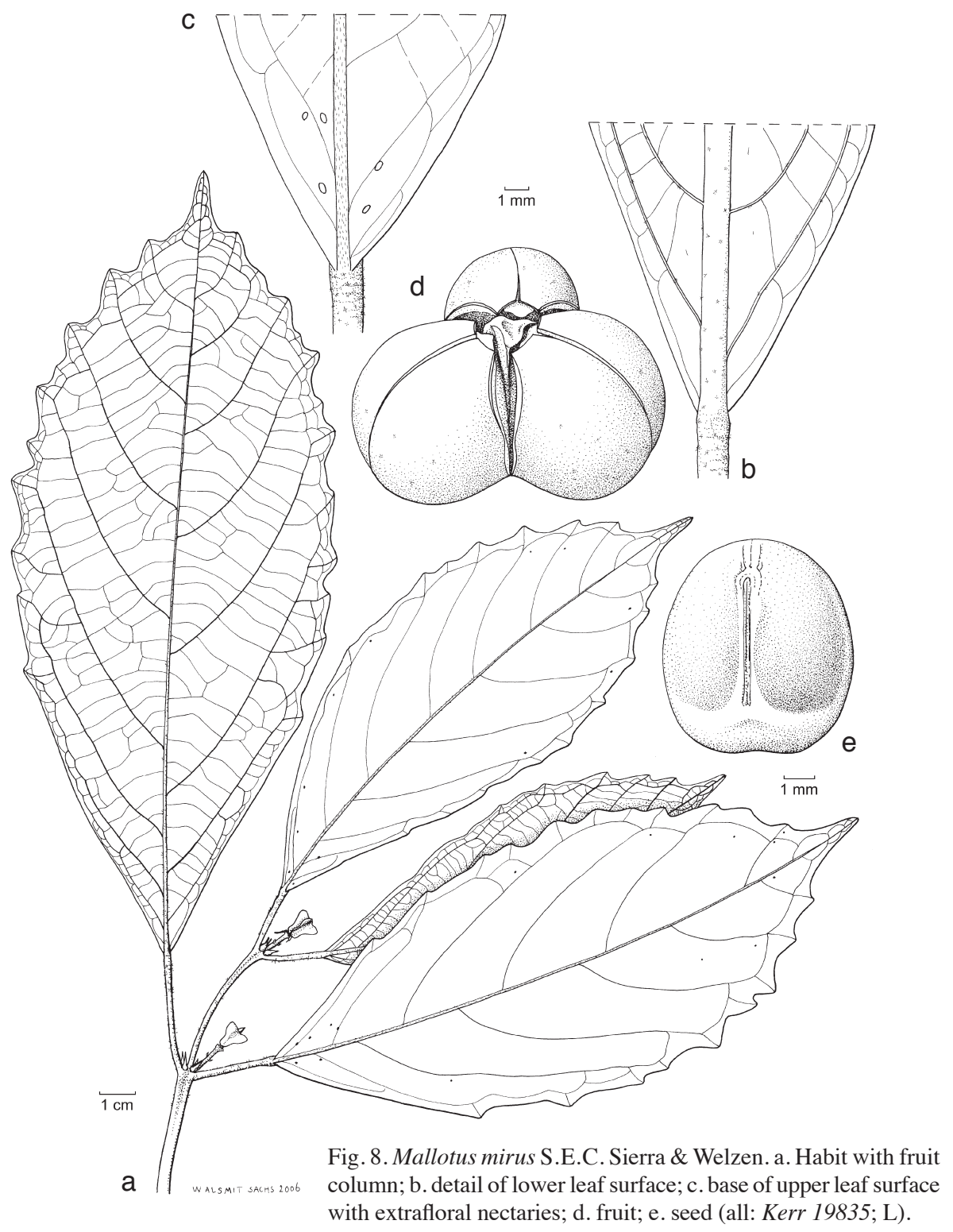




\section{Mallotus monanthos Airy Shaw - Map 1}

Mallotus monanthos Airy Shaw (1978a) 402. - Type: KEP FRI (Whitmore) 3544 (holo K; iso A,

L), Peninsular Malaysia, Pahang, Krau Game Reserve, Kuala Lumpat.

Small trees, 2.4-5 m high, monoecious (probably also dioecious, see note). Indumentum composed of simple and tufted hairs, glandular hairs absent. Stipules linear-triangular, 9-11 by $1.5-2 \mathrm{~mm}$, caducous, margin entire, apex acute, scatteredly hairy outside, densely hairy inside. Leaves opposite; petiole $5-15$ by $2.5-4 \mathrm{~mm}$, densely hairy; blade narrowly obovate, $25-42$ by $7-11.5 \mathrm{~cm}$, length/width ratio $3.4-4$, coriaceous, base attenuate, margin entire, undulate, apex caudate, upper surface glabrous, larger extrafloral nectaries marginal, 2 or 3 per side, $0.5-2.5 \mathrm{~mm}$ from margin, elliptic to orbicular, $0.8-1$ by $0.7-0.8 \mathrm{~mm}$, smaller ones from base to apex, $9-13$ per side, $4-7 \mathrm{~mm}$ from margin, orbicular, $0.2-0.3$ by $0.2-0.3 \mathrm{~mm}$, lower surface glabrous, domatia absent, venation pinnate, nerves 15-18 per side, looping and closed near margin. Inflorescences erect, several together; peduncle absent; bracts linear-triangular, persistent, margin entire, apex acute, hairy, sparsely hairy to glabrous outside, sparsely hairy inside; bracteoles absent. Flowers: pedicels densely hairy; sepals free, persistent, margin entire, apex acute, densely hairy outside, glabrous inside. Staminate inflorescences (only bracts and flowers seen): bracts numerous, c. $40,3-4$ by $0.4-0.5 \mathrm{~mm}$. Staminate flowers $4-5 \mathrm{~mm}$ diam.; pedicels $1-1.7 \mathrm{~mm}$ long; sepals 3 , ovate, $5-8.5$ by $1.5-3 \mathrm{~mm}$; stamens $20-23$, glabrous, filaments $2-5 \mathrm{~mm}$ long, basally connate, thecae ellipsoid, $0.6-0.7$ by $0.2-0.25$ $\mathrm{mm}$, connective widened; pistillode present. Infructescences reduced to 1 terminal fruit, $1 \mathrm{~cm}$ long; peduncle reduced; bracts c. $15,8-10$ by $1.3-1.5 \mathrm{~mm}$. Pistillate flowers (based on young fruit): pedicels c. $3 \mathrm{~mm}$ long; sepals 3 , narrowly triangular, c. 7 by 1.8 $\mathrm{mm}$; ovary 3-locular, densely hairy; style c. $3 \mathrm{~mm}$ long; stigmas c. 8 by $1 \mathrm{~mm}$. Fruits capsules, c. 17 by $20 \mathrm{~mm}$, opening septicidally-loculicidally, surface smooth, densely hairy; wall c. $1 \mathrm{~mm}$ thick, glabrous inside; column c. 4 by $2 \mathrm{~mm}$. Seeds \pm globose, c. 9 by 9.5 by $9.3 \mathrm{~mm}$, surface smooth, dull, light cream brown.

Distribution - Endemic to Peninsular Malaysia.

Habitat \& Ecology - Dense lowland forest. Altitude $365 \mathrm{~m}$. Flowering and fruiting: March.

Note - The two collections studied (KEP FRI 3544 and Soepadmo 736) have labels that only refer to the fruits. Soepadmo 736 (A) consists of a loose fruit. KEP FRI $3544(\mathrm{~A}, \mathrm{~K})$ also consists of fruits, while the $\mathrm{L}$ specimen has two branches, one with female (longer and few) and the other with male bracts (shorter and numerous), with the staminate flowers stored in an envelope. Based on KEP FRI 3544 (L) it is likely that the species is monoecious (if the branches belong to the same plant), while based on Soepadmo 736 (A) it may be dioecious; it is therefore evident that further material needs to be studied.

\section{Mallotus montanus (Wall. ex Müll.Arg.) Airy Shaw - Map 8}

Mallotus montanus (Wall. ex Müll.Arg.) Airy Shaw (1977) 78; Welzen et al. (2000) 102 - - [Croton montanus: Wall. (1845?) 7723B, nom. nud., non Willd.] - Coelodiscus montanus Wall. ex Müll. Arg. in DC. (1866) 759. - Lectotype (selected here): Wallich 7723B (holo G-DC (IDC microfiche 800); iso BM, E, K-W (IDC microfiche 7394), L), Peninsular Malaysia, Penang.

Mallotus eriocarpus auct. non Müll.Arg.: Pax \& K. Hoffm. (1914) 160; Whitmore (1973) 115; quoad specim. from Peninsular Malaysia. 
Shrubs to small trees, up to $6 \mathrm{~m}$ high, dioecious or rarely monoecious. Indumentum composed of simple, tufted, stellately-tufted and stellate hairs (on petiole and midrib of blade) and light yellow-orange glandular hairs. Stipules deltoid to triangular, 0.6-1.3 by $0.5-0.6 \mathrm{~mm}$, early caducous, margin entire, apex rounded, densely hairy. Leaves opposite; petiole $1-7$ by $0.5-2 \mathrm{~mm}$, densely hairy; blade ovate to elliptic, $5-24$ by $3-12$ $\mathrm{cm}$, length/width ratio 1.6-2.5, chartaceous, base cordate to obtuse, margin denticulate, with glandular teeth, apex acute to caudate, upper surface sparsely hairy (densely hairy on midrib and nerves), sometimes with glandular hairs, extrafloral nectaries marginal from base to apex 7-17 per side, $0.4-2 \mathrm{~mm}$ from margin, elliptic to orbicular, $0.2-0.4$ by $0.2-0.4 \mathrm{~mm}$, lower surface sparsely hairy, sparsely covered with glandular hairs, domatia usually present (towards the petiole insertion), venation triplinerved, nerves 4-7 per side, looping and closed near margin or ending in margin. Inflorescences racemes, single, erect, unisexual or rarely bisexual; peduncle $7-35$ by c. $1 \mathrm{~mm}$, densely hairy; bracts margin entire, apex acuminate, densely hairy; bracteoles absent. Flowers: pedicels densely hairy; sepals free, persistent, margin entire, apex acute, densely hairy outside, scatteredly hairy to glabrous inside, with glandular hairs outside. Staminate inflorescences $2.5-4 \mathrm{~cm}$ long, nodes per branch 5-9, with 2-4 flowers per bract. Staminate flowers 3-3.5 mm diam.; pedicels $1.2-1.5 \mathrm{~mm}$ long; sepals 3 (or 4), ovate, $1.5-2$ by 2-2.5 mm; stamens $20-30$, filaments $2-3 \mathrm{~mm}$ long, free, densely hairy, thecae ovoid to ellipsoid, $0.2-0.3$ by $0.2 \mathrm{~mm}$, hairy to glabrous, connective not widened; pistillode present. Pistillate inflorescences 3-7 cm long, nodes per branch 1-6, with 1 flower per bract. Pistillate flowers $4.5-5.5 \mathrm{~mm}$ diam.; pedicels $0.8-1 \mathrm{~mm}$ long; sepals 2 or 3 , triangular, $2.5-3$ by $1.3-1.5 \mathrm{~mm}$; ovary $1-1.2$ by $1.2-1.5 \mathrm{~mm}$, 3-locular, densely hairy, sparsely covered with glandular hairs; style $0.2-0.4 \mathrm{~mm}$ long; stigmas $1-2$ by $0.6-0.8 \mathrm{~mm}$. Fruits capsules, $6-13$ by $15-19 \mathrm{~mm}$, opening septicidally-loculicidally, surface smooth, densely hairy, sparsely covered with glandular hairs; wall c. $0.5 \mathrm{~mm}$ thick, sparsely hairy inside; column $4-10$ by $4.5-6 \mathrm{~mm}$. Seeds \pm globose, $4.2-6.5$ by $4.2-6.5$ by $4.2-6.2 \mathrm{~mm}$, surface smooth, shiny, light brown.

Distribution - Thailand, Peninsular Malaysia.

Habitat \& Ecology - Locally common in primary, secondary and evergreen forest; along edges of rubber plantations; on calcareous rock. Altitude 100-400 m. Flowering and fruiting: March to November.

Notes - 1. Mallotus montanus can easily be confused with M. ustulatus (Gagnep.) Airy Shaw, in particular because of the leaves: ovate, base cordate and margin with glandular teeth. The main differences are found in the hairs of midrib and veins, domatia, fruits and geographical distribution: midribs and veins densely hairy on the upper surface (sparsely hairy in M. ustulatus), domatia always present (absent in M. ustulatus), leaves slightly rough (very rough in M. ustulatus); fruits opening septicidallyloculicidally (loculicidally-septicidally in M. ustulatus), spines absent (present in M. ustulatus), lobes conspicuous (inconspicuous in $M$. ustulatus), seeds brown, large, $4.2-6.5$ by $4.2-6.5$ by $4.2-6.2 \mathrm{~mm}$ (yellowish cream, small, c. 3 by 4 by $4 \mathrm{~mm}$ in M. ustulatus). Mallotus montanus is found in Thailand and Peninsular Malaysia, while M. ustulatus is only found in Cambodia.

2. Additionally, M. montanus can also be confused with M. eriocarpus (Thwaites) Müll.Arg. in particular because of the leaves: slightly rough, margin with glandular teeth, number of marginal glands (5-9) and the septicidally-loculicidally fruit dehiscence. 
The main differences are found in the leaf base, hairs of the lower leaf surface, domatia, stamens, fruits and geographical distribution: leave base ovate (rounded in M. eriocarpus), midribs and veins densely hairy on the lower surface (sparsely hairy in $M$. eriocarpus), domatia always present (absent in M.eriocarpus), hairy filaments (glabrous in $M$. eriocarpus), fruit spines absent (present in $M$. eriocarpus); seeds brown, large, $4.2-6.5$ by $4.2-6.5$ by $4.2-6.2 \mathrm{~mm}$ (yellowish cream, small, c. 2 by 3 by $3 \mathrm{~mm}$ in $M$. eriocarpus). Mallotus montanus is found in Thailand and Peninsular Malaysia, while M. eriocarpus is found in Sri Lanka.

3. See also the note for M. subcuneatus.

\section{Mallotus oppositifolius (Geiseler) Müll.Arg. - Map 14}

Mallotus oppositifolius (Geiseler) Müll.Arg. (incl. var. genuinus Müll.Arg., nom. inval.) (1865) 194; in DC. (1866) 976; Prain (1913) 928. - Croton oppositifolius Geiseler (1807) 23. - Echinus oppositifolius (Geiseler) Baill. (1891) 977. - Type: Isert s.n. (holo C (IDC microfiche 2203: 28, III, 2)), Ghana, 'Guinea'.

Acalypha? dentata Schumach. \& Thonn. in Schumach., preprint (1827) 184. - Rottlera dentata (Schumach. \& Thonn.) Baill. (1861) 69. - Mallotus oppositifolius (Geiseler) Müll.Arg. forma dentatus (Schumach. \& Thonn.) Pax \& K. Hoffm. (1914) 159. - Ricinocarpus dentatus (Schumach. \& Thonn.) Kuntze ex Govaerts, Frodin \& Radcl.-Sm. (2000) 1121, 1396 (see note 1). - Type: Thonning s.n. (holo C (IDC microfiche 2203: 1, I, 7); iso P-JU, cf. Baillon (1861) n.v.), Ghana.

Claoxylon cordifolium Benth. in Hook.f. \& Benth. (1849) 506. - Lectotype (selected here): Vogel 11 (holo K), Nigeria, Quorra.

Mallotus oppositifolius (Geiseler) Müll.Arg. var. glabrata Müll.Arg. (1865) 194. - Mallotus oppositifolius (Geiseler) Müll.Arg. forma glabratus (Müll.Arg.) Müll.Arg. ex Pax \& K. Hoffm. (1914) 158. - Lectotype (selected here): Irving 153 (holo K), Nigeria, Yoruba, Abeokuta.

Mallotus oppositifolius (Geiseler) Müll.Arg. var. integrifolius Müll.Arg. (1865) 195; Prain (1913) 929. - Type: Pervillé 360 (holo G; iso K, L, P), Madagascar, Nosy Bé.

Mallotus oppositifolius (Geiseler) Müll.Arg. var. pubescens Pax (1897) 525. - Mallotus oppositifolius (Geiseler) Müll.Arg. forma pubescens (Pax) Pax \& K. Hoffm. (1914) 158. - Type: Newton 18 (holo B $\uparrow$; iso COI?), Angola, Aguda.

?Mallotus chevalieri Beille (1908) 76. - Syntypes: Chevalier 5089 (n.v.), Lower Guinea, Kassa; Chevalier 5582 (n.v.), Lower Guinea, Krébedjé; Chevalier 11022 (n.v.), Lower Guinea, Bangui (vide Prain, 1913: 928).

Mallotus oppositifolius (Geiseler) Müll.Arg. forma lindicus Radcl.-Sm. (1974) 437. - Mallotus oppositifolius Müll.Arg. var. lindicus (Radcl.-Sm.) Radcl.-Sm. (1984) 789. - Type: Semsei 634 (holo K; iso EA n.v.), Tanzania.

Mallotus oppositifolius (Geiseler) Müll.Arg. forma polycytotrichus Radcl.-Sm. (1985) 658. - Type: Müller 2467 (holo SRGH n.v.; iso PRE n.v.), Zimbabwe.

Shrubs to small trees, up to $12 \mathrm{~m}$ high, dioecious. Outer bark smooth. Indumentum composed of simple, tufted and stellately-tufted hairs and yellow, orange to red glandular hairs. Stipules triangular to narrowly triangular, $1.7-4$ by $0.5-0.8 \mathrm{~mm}$, caducous, margin entire, apex acute, densely to scatteredly hairy outside, scatteredly hairy inside. Leaves opposite (see note 2); petiole $15-120$ by $0.7-1.2 \mathrm{~mm}$, densely to sparsely hairy; blade broadly ovate to ovate, $2-17.5$ by $1.3-8 \mathrm{~cm}$, length / width ratio $1.5-3.2$, membranaceous, base cordate, truncate, rounded to obtuse, margin (shallowly) dentate to crenate, with glandular teeth, rarely 2-lobed at widest part of blade (see note 2), apex acuminate to caudate, upper surface sparsely to scatteredly hairy, glandular hairs absent or rarely present, extrafloral nectaries marginal, on lower half, 2-7 per side, $0.1-0.5 \mathrm{~mm}$ from margin, elliptic to orbicular, $0.2-0.7$ by $0.2-0.3 \mathrm{~mm}$, lower surface sparsely hairy, 
glabrescent, densely covered with glandular hairs, domatia present, venation triplinerved, nerves 4-7 per side, indistinctly looping and closed near margin or ending in margin. Inflorescences racemes, single, erect; axes densely to sparsely hairy; bracts triangular, $0.8-2$ by $0.8-1 \mathrm{~mm}$, margin entire, apex acuminate to caudate, densely hairy outside, glabrous inside; bracteoles absent. Flowers: pedicels densely hairy; sepals persistent, margin entire, apex acute, sparsely hairy outside, glabrous inside, with glandular hairs. Staminate inflorescences 3-21 cm long; peduncle 0.7-35 mm long; nodes per branch 27-47, with 1-7 flowers per bract. Staminate flowers $2-5 \mathrm{~mm}$ diam.; pedicels $1.5-4 \mathrm{~mm}$ long; sepals 4 or $5,2-2.3$ by $1-1.2 \mathrm{~mm}$, free or basally connate; stamens 40-55, glabrous, filaments $1-2 \mathrm{~mm}$ long, free, thecae ovoid, $0.2-0.3$ by $0.2-0.25$ $\mathrm{mm}$, connective not widened; pistillode absent. Pistillate inflorescences $6.4-12.5 \mathrm{~cm}$ long; peduncle 20-35 mm long; nodes per branch 8-62, with 1 (rarely 2) flowers per bract. Pistillate flowers 2.8-3 mm diam.; pedicels 1.5-3.8 mm long; calyx 4-6lobed, lobes narrowly triangular, $1.5-1.7$ by $0.7-0.8 \mathrm{~mm}$; staminodes rarely present; ovary $1.2-1.4$ by $1.3-1.5 \mathrm{~mm}, 3$ - (or 4-)locular, densely to scatteredly hairy, densely to sparsely covered with glandular hairs; style $0.2-0.6 \mathrm{~mm}$ long; stigmas $1.3-1.7$ by $0.5-0.8 \mathrm{~mm}$. Fruits capsules, $5-6$ by $6-9 \mathrm{~mm}$, opening septicidally-loculicidally, surface smooth or rarely scatteredly verrucose, warts up to c. $0.3 \mathrm{~mm}$ long, scatteredly to rarely densely hairy (see note 2), densely to sparsely covered with glandular hairs; wall c. $0.2 \mathrm{~mm}$ thick, sparsely hairy inside; column $2-2.8$ by $1-2 \mathrm{~mm}$. Seeds \pm globose, $3-3.3$ by $2.2-2.8$ by $2.5-3 \mathrm{~mm}$, surface smooth, shiny, ochre.

Distribution - From SW (Senegal to Liberia) to SE (Ethiopia to Tanzania) Africa and W Madagascar.

Habitat \& Ecology - Common in semi-deciduous, dry or wet evergreen forest, in moist edges of gallery forest, periodically inundated forests, savannah vegetation with

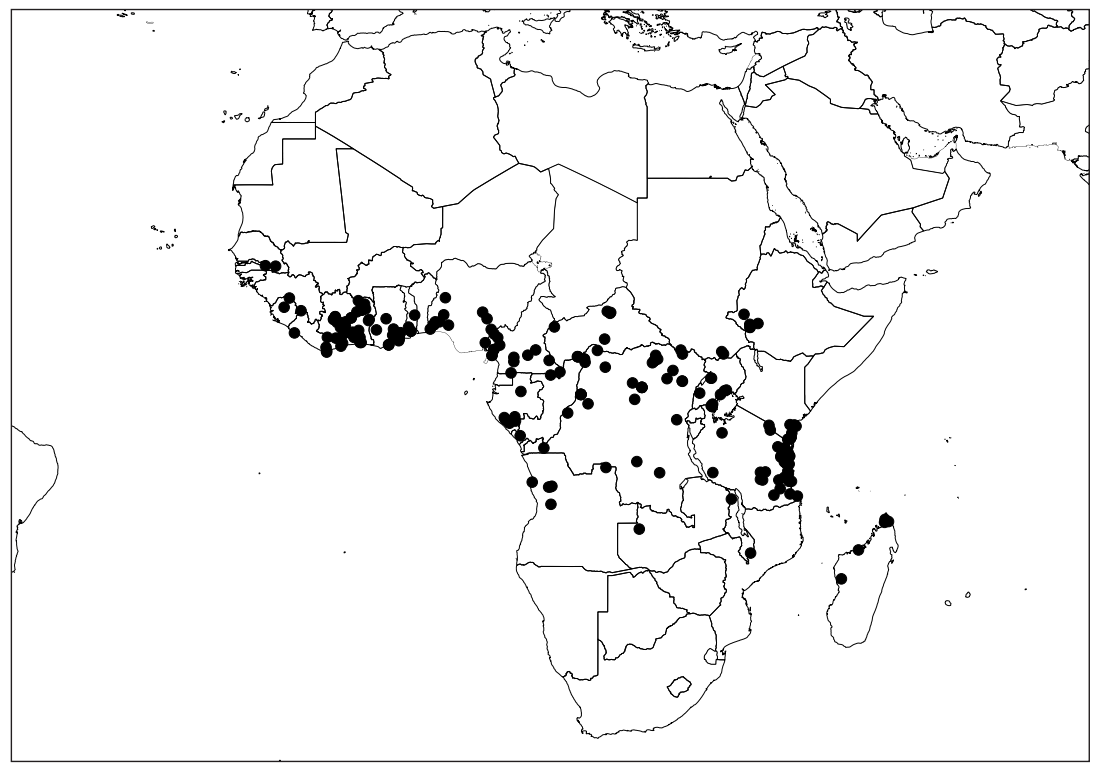

Map 14. Distribution of Mallotus oppositifolius (Geiseler) Müll.Arg. 
forest patches, in grasslands and swamps; in open and disturbed areas, on periphery of farms, along river edges; on limestone, sand loam, sand and clay soil. Altitude: sea level up to $1800 \mathrm{~m}$. Flowering and fruiting throughout the year.

Vernacular names - Congo D.R.: Amamambamamba. Sudan: Diabizo. Tanzania: Mbuni Mwitu (Bondei); Mchachamai, Nngaje (Kimatumbi); Mkungu riuza (Kizigua); Matarawanda, Mkumbanguruwe, Mtumbika, Sankulamnale (Kiswahili); Mkungu riuza (Kizigua). Central African Republic: Molo-Katabunga, Molo-Ndungbwa (Lissongo).

Uses - The plant is used as a living fence.

Notes - 1. Govaerts et al. (2000: 1121, 1396) cited a Ricinocarpus dentatus (Schumach. \& Thonn.) Kuntze but that name is not found in Kuntze (1891: 617), where there is a $R$. denudatus (Mull.Arg.) Kuntze, based on Acalaypha denudata Müll.Arg.

It is likely that Govaerts et al.'s combination is a misprint for $R$. denudatus, which they do not mention. The basionym clearly is Acalypha dentata Schumach. \& Thonn., and Govaerts et al. have made an invalid new combination, as the basionym is not fully cited.

2. Mallotus oppositifolius can be very variable in its leaf and fruit morphology. Enti 17 (from Ghana) has alternate and opposite leaves (usually only opposite). Bidgood et al. 1807, Milne-Redhead \& Taylor 7576, Semsei 634, Vollesen MRC 3171 (from Tanzania) have leaves that are 2-lobed at the widest part of the blade (usually denticulate, dentate or crenate). Abeid 803, Gereau 1529 (from Tanzania) and Barter 1742 (from Nigeria) have fruits with a densely hairy surface (usually glandular).

3. Mallotus oppositifolius has a pronounced midrib on the upper surface of the leaves.

\section{Mallotus pierrei (Gagnep.) Airy Shaw - Map 10}

Mallotus pierrei (Gagnep.) Airy Shaw (1968) 380; (1972) 304; Welzen et al. (2000) 106. - Coelodiscus pierrei Gagnep. (1923) 51. - Lectotype (selected here): Pierre 6254 (holo P), Vietnam, Baria, Mont Dinh.

Shrubs, up to $3 \mathrm{~m}$ high, dioecious or monoecious. Indumentum composed of simple, tufted and stellately-tufted hairs and yellow-orange glandular hairs. Stipules narrowly triangular to linear-triangular, $1.6-5.5$ by $0.5-1.2 \mathrm{~mm}$, persistent to caducous, margin entire, apex acute, sparsely hairy outside, scatteredly hairy inside. Leaves opposite to rarely alternate; petiole $13-145$ by $1-1.8 \mathrm{~mm}$, densely hairy; blade broadly ovate to elliptic, $2.5-25$ by $1.5-15 \mathrm{~cm}$, length/width ratio $1-2.8$, membranaceous to chartaceous, base truncate, rounded, obtuse to cuneate, margin denticulate, with glandular teeth, apex acute to acuminate, upper surface sparsely to scatteredly hairy, densely hairy on midrib and nerves, extrafloral nectaries marginal on lower half, 1-6 per side, $0.3-3 \mathrm{~mm}$ from margin, elliptic, $0.4-0.8$ by $0.2-0.5 \mathrm{~mm}$, lower surface sparsely hairy, scatteredly covered with glandular hairs (sometimes only present near the leaf margin), domatia absent, venation triplinerved, nerves 4-7 per side, ending in margin or looping and closed near margin. Inflorescences racemes (staminate ones sometimes branching), single, erect; axes densely to sparsely hairy; peduncle $5-50$ by $0.5-1 \mathrm{~mm}$; bracts: margin entire or trilobed, apex acute, sparsely hairy outside, scatteredly hairy to glabrous inside; bracteoles $1.4-3$ by $0.4-0.5 \mathrm{~mm}$, persistent, margin entire, apex acute. Flowers: pedicels densely hairy; sepals free, persistent, margin entire, apex acute, 
densely hairy outside, glabrous inside, glandular hairs absent. Staminate inflorescences 6-12 cm long, nodes per branch 3-7, with 4-6 flowers per bract; bracts linear-triangular, 7-12 by $0.6-0.7 \mathrm{~mm}$, entire. Staminate flowers $4-7 \mathrm{~mm}$ diam.; pedicels $2.5-3.5$ mm long; sepals 3 or 4, ovate to elliptic, $2.3-3.1$ by $0.8-1.3 \mathrm{~mm}$; stamens $19-35$, filaments $1-3 \mathrm{~mm}$ long, free, sparsely hairy, thecae ellipsoid, $0.2-0.3$ by $0.2-0.25$ $\mathrm{mm}$, scatteredly hairy to glabrous, connnective widened; pistillode present. Pistillate inflorescences 3-15 cm long, nodes per branch 1-8, with 1 flower per bract; bracts triangular, 2-3 by $0.7-2 \mathrm{~mm}$, margin entire or trilobed. Pistillate flowers $2.6-3.2 \mathrm{~mm}$ diam.; pedicels 4-12 mm long; sepals 4 or 5, narrowly triangular, 2-4.8 by $0.6-0.9$ $\mathrm{mm}$; ovary $1.5-3$ by $1.4-2.2 \mathrm{~mm}$, (2- or) 3- (or 4-)locular, sparsely hairy, scatteredly covered with glandular hairs; style $0.5-1 \mathrm{~mm}$ long; stigmas $2-3$ by $0.5-0.8 \mathrm{~mm}$. Fruits capsules, 5-7 by 7-12 mm, opening septicidally-loculicidally, surface spiny, densely hairy, scatteredly covered with (inconspicuous) glandular hairs; spines 75-120, slightly curved, $0.8-1.2$ by c. $0.1 \mathrm{~mm}$, sparsely hairy; wall $0.8-1 \mathrm{~mm}$ thick, sparsely hairy to glabrous inside; column $4.5-5.8$ by $4-5 \mathrm{~mm}$. Seeds \pm globose, $4-5.5$ by $4.5-5$ by 4-5 mm, surface smooth, dull, light brown.

Distribution - Thailand, Vietnam.

Habitat \& Ecology - In scrub and evergreen, deciduous and mixed evergreen and deciduous forest; in very disturbed secondary growth, in shaded and open areas, on hills and along trails; on granite and limestone soil. Altitude: sea level to $930 \mathrm{~m}$. Flowering and fruiting throughout the year.

Vernacular names - Thailand: Kong kang, ton kí tae (Thai).

Note - Mallotus pierrei resembles M. ustulatus but differs in having leaves with truncate, rounded, obtuse to cuneate base (cordate in M. ustulatus), and acute or acuminate apex (rounded, obtuse or acute in M.ustulatus); hairy filaments (glabrous in M.ustulatus), long stigmas (short in M. ustulatus), ovaries densely hairy (sparsely hairy in M. ustulatus), fruits with few spines (many in M. ustulatus).

\section{Mallotus resinosus (Blanco) Merr. - Fig. 9; Map 15}

Mallotus resinosus (Blanco) Merr. (1918) 222; (1923) 436; Airy Shaw (1972) 294; (1975) 161; (1980b) 656; (1982) 29. - Adelia resinosa Blanco (1845) 562. - Neotype (Balakrishnan \& Chakrabarty 1991: 37): Merrill Species Blancoanae 485 ('483') (holo US; iso A, L, NY, P), Philippines, Luzon, Batangas, Mount Batulao.

Claoxylon muricatum Wall. ex Wight (1852) 24, t. 1886. - [Croton muricatus ('muricatum') Wall. (1845?), nom. nud.]. - Rottlera muricata (Wight) Thwaites (1861) 273. - Mallotus muricatus (Wight) Müll.Arg. (1865) 191. - Mallotus muricatus (Wight) Bedd. (1873) ccviii, pro nov., isonym. - [Mallotus muricatus (Wight) Müll.Arg. var. genuinus Pax \& K. Hoffm. (1914) 190, nom. inval.] - Coelodiscus muricatus (Wight) Gagnep. (1925) 369, pro comb. - Lectotype (selected here): Wight KD 2672 (holo K, marked 'A'; iso A, C n.v., L), India, 'Courtallum, July 1835, in Herb. Ind. Or. Hook.f.'.

Mallotus dispar (Blume) Müll.Arg. var. psiloneurus Müll.Arg. (1865) 191. - Type: Zollinger 3804 (holo G barcode G00011915; iso G barcode G00011934, K, L), Java.

Mallotus stenanthus Müll.Arg. (1865) 191. - Lectotype (selected here): Wallich 7751 B (holo G-DC n.v., fide IDC microfiche 800; iso K-W n.v., photo seen, IDC microfiche 7394), India, 'Courtallum, Herb. Madras'.

Mallotus walkerae Hook.f. (1887) 437. - Mallotus muricatus (Wight) Müll.Arg. var. walkerae (Hook.f.) Pax \& K. Hoffm. (1914) 190. — Lectotype (Philcox, 1997: 150): Walker s.n. (holo K), Sri Lanka. 
Mallotus walkerae Hook.f. var. laxiflora Hook.f. (1887) 437. - Type: Walker 1217 (holo K), Sri Lanka.

Mallotus spec. nov. 2: Welzen et al. (2000) 110. - Mallotus viridis Welzen \& Chayam. in Smitinand (2001) 343, nomen; Welzen \& Chayam. (2001) 652, f. 2. - Type: Santisuk 6684 (holo BKF), Thailand, South-eastern, Chanthaburi, Soi Dao Nua.

Mallotus muricatus auct. non Müll.Arg.: Kurz (1877) 384, 'excl. synon.', fide Hooker f., see next. - Mallotus andamanicus Hook.f. (1887) 439. - Lectotype (selected here): Kurz s.n. (holo CAL n.v.; iso CAL, L, see note 1), S Andaman Islands.

Shrubs to small trees, up to $8 \mathrm{~m}$ high, dioecious. Indumentum composed of simple and tufted hairs and light yellow to yellow-orange glandular hairs. Stipules triangular to narrowly triangular, $2.3-4.3$ by $1-1.7 \mathrm{~mm}$, early caducous, margin subentire, apex acute to rounded, scatteredly hairy outside, glabrous inside. Leaves opposite; petiole $2.5-20(-35)$ by $0.8-1.3 \mathrm{~mm}$, sparsely to scatteredly hairy; blade elliptic to obovate, $3.5-25$ by $1.7-10.7 \mathrm{~cm}$, length/width ratio $1.7-4.3$, chartaceous, base obtuse, cuneate to shallowly attenuate, margin denticulate, dentate to crenate, with glandular teeth, apex acute to caudate, upper surface glabrous, usually scatteredly covered with glandular hairs, extrafloral nectaries touching the midrib to marginal in lower half, 1 or 3(-5) per side, $1-3 \mathrm{~mm}$ from margin, elliptic, $0.5-3.5$ by $0.3-1 \mathrm{~mm}$, lower surface densely (to sparsely) covered with glandular hairs, domatia absent, venation pinnate, nerves 8-12 per side, looping and closed near margin or ending in margin. Inflorescences racemes, single, erect; peduncle 3-7 by $1-1.2 \mathrm{~mm}$; axes densely hairy; bracts ovate or deltoid to triangular, $1.2-2.5$ by $1-1.6 \mathrm{~mm}$, persistent or caducous, margin subentire, apex acute to acuminate, sparsely to scatteredly hairy outside, glabrous inside; bracteoles absent. Flowers: pedicels densely to sparsely hairy; sepals persistent, margin entire, apex acute, light brown with glandular hairs outside. Staminate inflorescences 1.7-17.3 $\mathrm{cm}$ long, nodes per branch 7-39, with 3-7 flowers per bract. Staminate flowers $2.5-4$ mm diam.; pedicels $2-5 \mathrm{~mm}$ long; sepals 3 or 4, elliptic to obovate, $1.5-2.3$ by $1.2-1.3$ $\mathrm{mm}$, free, sparsely hairy outside, glabrous inside; stamens $29-40$, filaments $1.5-2.5$ $\mathrm{mm}$ long, free, glabrous, thecae ellipsoid, $0.2-0.3$ by $0.2-0.25 \mathrm{~mm}$, hairy, connnective widened; pistillode present. Pistillate inflorescences $2.1-7.2 \mathrm{~cm}$ long, nodes per branch 6-10, with 1 flower per bract. Pistillate flowers $1.5-2 \mathrm{~mm}$ diam.; pedicels $0.5-1 \mathrm{~mm}$ long; calyx 4-6-lobed, lobes narrowly triangular, $2.7-3$ by $0.7-0.8 \mathrm{~mm}$, basally connate, scatteredly hairy to glabrous outside, glabrous inside; ovary $1.3-1.7$ by $1.4-1.9$ $\mathrm{mm}, 3$-locular, sparsely covered with glandular hairs; style $0.2-0.5 \mathrm{~mm}$ long; stigmas $2-3.5$ by $1 \mathrm{~mm}$. Fruits capsules, $6-8$ by $8-15 \mathrm{~mm}$, opening septicidally-loculicidally, surface spiny, scatteredly hairy to glabrescent, densely to sparsely covered with glandular hairs; spines $40-80$, straight to curved (see note), $1-2.2$ by $0.3-0.5 \mathrm{~mm}$, scatteredly hairy to glabrous; wall $0.7-1 \mathrm{~mm}$ thick, sparsely hairy to glabrous inside; column $4-6$ by $4-5 \mathrm{~mm}$. Seeds \pm globose, $5-8$ by $4-6.5$ by $4-6 \mathrm{~mm}$, surface smooth, shiny, brown to brown-grey.

Distribution - S India, Sri Lanka, Andaman \& Nicobar Islands, Thailand, Laos, Vietnam, Philippines, Borneo, Java, Lesser Sunda Islands, Sulawesi, Moluccas, Papua New Guinea, Australia.

Habitat \& Ecology - Locally in understorey of mixed evergreen deciduous, secondary and notophyll vine forest and grassland; near roads, near streams and sea; on clay, granite, loam, limestone, sandstone or volcanic soil. Altitude: sea level up to $1000 \mathrm{~m}$. Flowering and fruiting throughout the year. 
Fig. 9. Mallotus resinosus (Blanco) Merr. a. Habit with fruit columns; b. base of upper leaf surface with extrafloral nectaries; c. fruit and seed (all: Santisuk 6684; BKF).

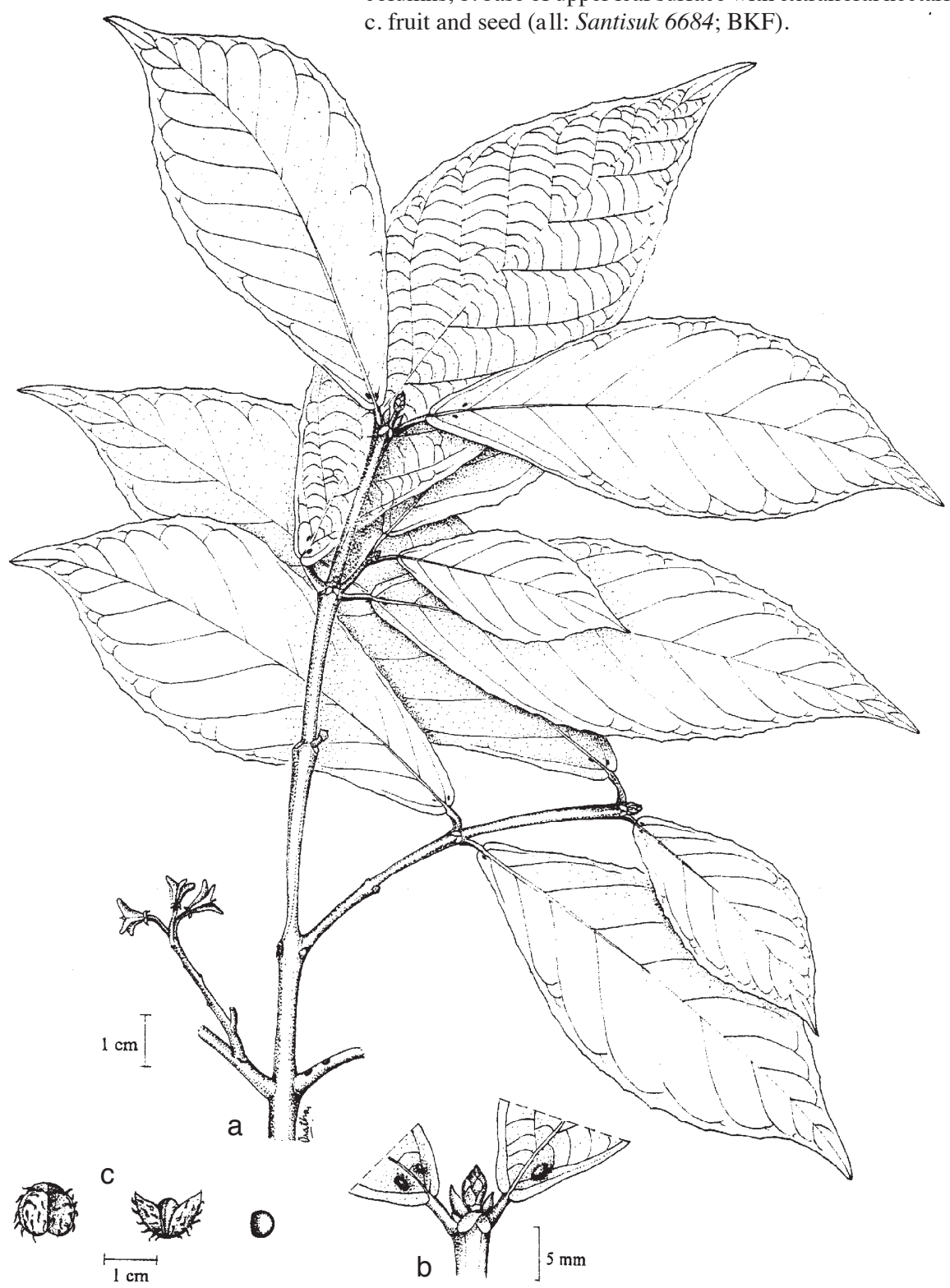

Vernacular names - Philippines: Agudín (Dum), hangbi táwad (Mango), tibalbagyon (Tagbanua). Moluccas: O hararoko (Tobelorese). Lesser Sunda Islands: Anak wati, mboréng.

Notes - 1. Balakrishnan \& Chakrabarty (1991) report the presence of four Kurz collections at CAL, unfortunately without date and sex or fruiting stage. A male fruiting 


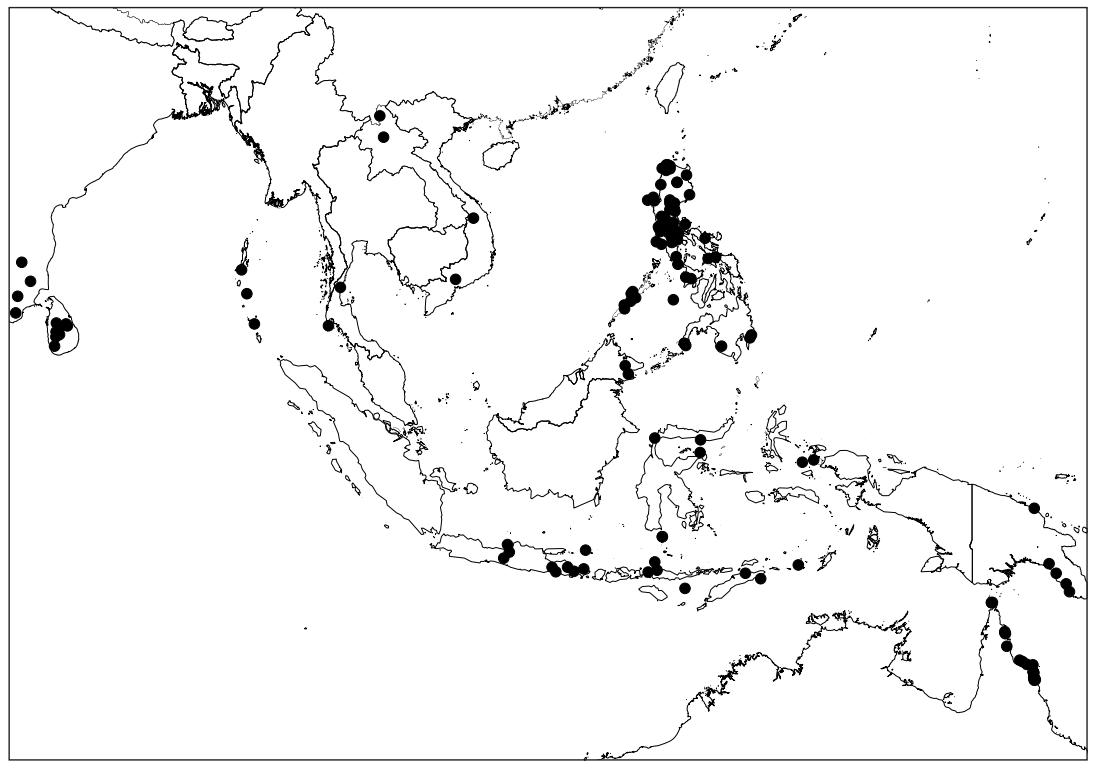

Map 15. Distribution of Mallotus resinosus (Blanco) Merr.

specimen collected by Kurz is at L and has been appointed as lectotype. Because the material from CAL has not been studied, we do not know exactly how many sheets bearing male specimens there might be. In $\mathrm{K}$ there is no accepted specimen labelled as $M$. andamanicus.

2. Mallotus resinosus has a pronounced midrib on the upper surface of the leaves.

3. There have been several names published for Indian collections that show similarities with $M$. resinosus, but their status can only be determined in a detailed taxonomic study that includes all type material and more collections from that region. As an aid in this we here provide some data on their nomenclature and typification.

Mallotus lawii Müll.Arg. (1865) 192. - Type: Stocks \& Law 'in Hook.f. \& Thoms. Hb. Ind. Or. Claoxyl. 6' (holo G-DC (IDC microfiche 800); iso L, NY), India, Malabar.

Mallotus aureo-punctatus Müll.Arg. in DC. (1866) 973. - Rottlera aureopunctata Dalzell (1851) 122; Dalzell \& A. Gibson (1861) 230. - Syntypes: Unknown s.n. (CAL?, DD?, K?, W?), India, shady jungles in the Ghauts (Ghats), hilly parts, Concan; Meera Hills. 'Noticed in Graham's list under 1334'.

Mallotus intermedius (Baill.) N.P. Balakr. (1968) 245. - Axenfeldia intermedia Baill. (1858) 419. - Syntypes: Unknown s.n. (P n.v.), India.

Mallotus subramanyamii J.L. Ellis (1983) 199. - Mallotus resinosus (Blanco) Merr. var. subramanyamii (J.L. Ellis) Chakrab. (1985) 704. - Type: J.L. Ellis 34963 (holo CAL n.v.; iso MH n.v.), India, Tamil Nadu, Nilgiri Hills, Moolakadu, Ouchterlong Valley.

4. See also note 3 for M.decipiens.

\section{Mallotus rufidulus (Miq.) Müll.Arg. - Map 6}

Mallotus rufidulus (Miq.) Müll.Arg. in DC. (1866) 970. - Rottlera rufidula Miq. (1861) 181, nom. nud.; 453. - Type: Diepenhorst HB 2510 (holo U), Sumatra, Priaman. 
Mallotus zollingeri Müll.Arg. (1865) 193. - Type: Zollinger 1098* (holo G, barcode G00016017; iso BM, G, P), Java.

Rottlera dispar auct. non Blume (1826): Moritzi (1846) 17 (nomen); Rchb.f. \& Zoll. (1856) 17 (descr.!). - Mallotus moritzianus Müll.Arg. (1865) 190. - Mallotus moritzianus Müll.Arg. var. scaber Müll.Arg. (1865) 190, nom. inval. - Rottlera moritziana (Müll.Arg.) Scheff. (1869) 125. - Lectotype (selected here): Zollinger 1616 (holo G, barcode G00011931; iso BM, FI, G, P, ZT), Java.

Shrubs to small trees, up to $6 \mathrm{~m}$ high, dioecious or rarely monoecious. Outer bark smooth. Indumentum composed of simple and stellately-tufted hairs and yellow to orange glandular hairs. Stipules narrowly triangular, $2.8-7$ by $0.7-1 \mathrm{~mm}$, early caducous, margin entire, apex acute, sparsely hairy. Leaves opposite; petiole $8-70$ by $0.7-1.2 \mathrm{~mm}$, densely to sparsely hairy; blade ovate to elliptic, $8-24$ by $3.8-17 \mathrm{~cm}$, length/width ratio 1.6-3, membranaceous to chartaceous, base rounded, obtuse to cuneate, margin entire, rarely dentate, sometimes with glandular teeth, apex caudate, upper surface densely to sparsely hairy on midrib and nerves, extrafloral nectaries marginal throughout blade, up to 22 per side, 2-10 $\mathrm{mm}$ from margin, sometimes also all over the blade, elliptic to orbicular, $0.2-0.5$ by $0.2-0.5 \mathrm{~mm}$, lower surface sparsely to scatteredly hairy, sparsely covered with glandular hairs, domatia absent, venation triplinerved, nerves 4-6 per side, looping and closed near margin. Inflorescences racemes, single, erect, unisexual or rarely bisexual; axes sparsely hairy; peduncle $20-80$ by $1-1.2 \mathrm{~mm}$; bracts triangular, $1-4.2$ by $0.7-1.3 \mathrm{~mm}$, persistent to caducous, margin entire, apex acute, densely to sparsely hairy; bracteoles triangular, $0.5-0.7$ by $0.2-0.3 \mathrm{~mm}$, early caducous. Flowers: pedicels densely hairy; sepals persistent, margin entire, apex acute, densely hairy and with few glandular hairs outside, glabrous inside. Staminate inflorescences 9-20 cm long, nodes per branch 6-13, with 5-10 flowers per bract. Staminate flowers 3-4.5 mm diam.; pedicels 2.5-5 mm long; sepals 3 (or 4), triangular, 2.9-3.1 by $1.8-2$, free; stamens 50-60, glabrous, filaments $1.5-3.2 \mathrm{~mm}$ long, free (to slightly connate at the base), thecae ovoid, $0.2-0.3$ by $0.2-0.25 \mathrm{~mm}$, connective not widened; pistillode present. Pistillate inflorescences 16-20 cm long, nodes per branch 2-8, with 1 flower per bract. Pistillate flowers 4-5 mm diam.; pedicels 3-8 $\mathrm{mm}$ long; calyx 3-lobed, lobes narrowly triangular, $5-6$ by $1-1.4 \mathrm{~mm}$, basally connate; ovary $2.5-3$ by 2.5-3 mm, 3-locular, densely hairy, glandular hairs scattered; style 0-1(-2) mm long; stigmas $3-4$ by $1-1.3 \mathrm{~mm}$. Fruits capsules, $8-20$ by $7-19 \mathrm{~mm}$, green, opening septicidally-loculicidally, surface spiny, densely hairy, scatteredly covered with glandular hairs; spines $200-250, \pm$ straight, $2.8-4$ by $0.2-0.3 \mathrm{~mm}$, densely hairy; wall $0.8-1.1$ $\mathrm{mm}$ thick, glabrous inside; column $7-8$ by $7-8 \mathrm{~mm}$. Seeds \pm globose, $6.5-9$ by $6-9$ by $6-9 \mathrm{~mm}$, surface smooth, dull, light cream-brown.

Distribution - Sumatra, Java, Borneo, Lesser Sunda Islands.

Habitat \& Ecology - Riverine forest; on limestone. Altitude 30-750 m. Flowering and fruiting: June to November.

Vernacular names - Sumatra: Kalikih utan. Java: Kaijae tekeh, waru rot. Lesser Sunda Islands: Tjempa, Pampa.

Notes -1 . See also the note for M. connatus.

2. Kostermans s.n. (L barcode L0294643) has two labels. One is a field label with the locality 'Ujung Kulon' (Java). The other one is a typed label with the locality 'Berau near Tgd. Redeb, E Indonesia, Borneo'. We think that the collecting locality is more likely to be in Java. 


\section{Mallotus sphaerocarpus (Miq.) Müll.Arg. - Map 3}

Mallotus sphaerocarpus (Miq.) Müll.Arg. in DC. (1866) 976. - Rottlera sphaerocarpa Miq. (1861) 454. - Type: Diepenhorst HB 2119 (holo U; iso G-DC, fragm., fide microfiche IDC 800; BO ('Teysmann')), Indonesia, Sumatra, Priaman.

Small to large trees, $8-30 \mathrm{~m}$ high, dbh $10-35 \mathrm{~cm}$, dioecious. Indumentum composed of stellately-tufted hairs and yellow to orange glandular hairs. Stipules narrowly triangular, $4.8-7$ by $0.8-1.2 \mathrm{~mm}$, early caducous, margin entire, apex acute, densely hairy. Leaves opposite to rarely alternate; petiole $56-80(-130)$ by $1.8-3 \mathrm{~mm}$, densely to sparsely hairy; blade ovate to elliptic, sometimes slightly falcate, $12-32$ by $10-18$ $\mathrm{cm}$, length/width ratio 1.4-2, membranaceous to chartaceous, base obtuse to cuneate, margin denticulate to crenulate (rarely dentate), with glandular teeth, apex cuspidate to caudate, upper surface scatteredly hairy to glabrous, larger extrafloral nectaries basal near the petiole insertion, $1-3$ per side, elliptic, $0.5-1.5$ by $0.2-0.7 \mathrm{~mm}$, smaller ones marginal in upper half, $8-13$ nectaries per side, $1-3.5 \mathrm{~mm}$ from margin, orbicular, $0.2-0.3$ by $0.2-0.3 \mathrm{~mm}$, lower surface sparsely hairy, scatteredly covered with glandular hairs (sometimes only present towards the leaf margin), domatia absent, venation triplinerved, nerves $6-10$ per side, looping and closed near margin. Inflorescences racemes, single, erect; axes densely hairy; peduncle $5-10$ by $1-2 \mathrm{~mm}$; bracts triangular, $1.5-3$ by $0.5-1 \mathrm{~mm}$, persistent to caducous, margin entire, apex caudate, densely hairy; bracteoles absent. Flowers: pedicels densely hairy; sepals persistent, margin entire, apex acute, densely hairy outside, glabrous inside, glandular hairs absent. Staminate inflorescences $16-21 \mathrm{~cm}$ long; nodes per branch 63-100, with 6-13 flowers per bract. Staminate flowers 3-3.4 mm diam.; pedicels 2-7 mm long; sepals 3, ovate to elliptic, $2-3$ by $1.7-2.1 \mathrm{~mm}$, free; stamens $30-38$, glabrous, filaments $0.3-1.8 \mathrm{~mm}$ long, flattened, free, thecae ovoid, $0.1-0.2$ by $0.1-0.2 \mathrm{~mm}$, connective umbrella-like; pistillode absent. Pistillate inflorescences $9-14 \mathrm{~cm}$ long, nodes per branch 12-60, with 1 flower per bract. Pistillate flowers 4-5 $\mathrm{mm}$ diam.; pedicels $0.8-1.2 \mathrm{~mm}$ long; calyx $3-5$-lobed, lobes triangular, $2.5-3$ by $1.2-2 \mathrm{~mm}$, basally connate; ovary $2-2.2$ by $2-2.2 \mathrm{~mm}, 3$-locular, densely hairy, glandular hairs absent; style $0.4-0.7 \mathrm{~mm}$ long; stigmas $1.8-3$ by $1-1.4 \mathrm{~mm}$. Fruits indehiscent, spheroid, $15-19$ by $15-19 \mathrm{~mm}$, with 3 longitudinal ridges when dry, surface slightly verrucose, warts $20-100,0.5-1$ by $0.2-0.3 \mathrm{~mm}$, densely hairy, glandular hairs rarely present; wall: woody part c. $0.1 \mathrm{~mm}$ thick, endocarp fleshy; column absent. Seeds lenticular, $7-10.5$ by $6-7.5$ by $4-5 \mathrm{~mm}$, surface rugose, dull, black.

Distribution - Endemic to Sumatra.

Habitat \& Ecology - Locally common in disturbed secondary forest, also found in primary forest; in open areas; on sandy clay soil. Altitude 50-500 m. Flowering: February to March; fruiting: April to October. The fruits are eaten by orang-utans.

Vernacular names - Sumatra: Pepuah (Gajo); rumpi rawan.

Notes -1 . Koop 130 and 200 are atypical in having membranaceous, dentate and opposite to alternate leaves.

2. See also note 1 for M. blumeanus. 


\section{Mallotus subcuneatus (Gage) Airy Shaw - Map 12}

Mallotus subcuneatus (Gage) Airy Shaw (1972) 304; Whitmore (1973) 115; Welzen et al. (2000) 109. - Coelodiscus subcuneatus Gage (1922) 240. - Lectotype (selected here): Robinson \& Kloss 6173 (holo K; iso SING), Peninsular Malaysia, Kedah, Langkawi. Mallotus dispar auct. non. (Blume) Müll.Arg. (1865): Airy Shaw (1968) 380.

Shrubs to small trees, up to $8 \mathrm{~m}$ high, dioecious. Indumentum composed of simple, tufted, stellately-tufted and rarely peltate hairs and yellow-orange glandular hairs. Stipules linear-triangular, 3-4 by $0.4-0.6 \mathrm{~mm}$, early caducous, margin entire, apex acute, densely hairy. Leaves opposite; petiole $30-62$ by $1-1.5 \mathrm{~mm}$, densely hairy; blade ovate to elliptic, $7-20$ by $4.5-8 \mathrm{~cm}$, length/width ratio $1.5-2.5$, membranaceous to chartaceous, base rounded, obtuse to cuneate, margin denticulate, with distinct glandular teeth, apex (acute to) cuspidate, upper surface densely hairy to glabrous on midrib and nerves, extrafloral nectaries marginal in lower half, 1-10 per side, $1-10 \mathrm{~mm}$ from margin, elliptic to orbicular, $0.2-0.7$ by $0.2-0.3 \mathrm{~mm}$, lower surface sparsely hairy, sparsely covered with glandular hairs, domatia absent, venation triplinerved, nerves 4-6 per side, looping and closed near margin or ending in margin. Inflorescences racemes single, erect; axes densely hairy; bracts narrowly to linear-triangular, $2.5-3$ by $0.7-1$ $\mathrm{mm}$, persistent, margin entire, apex acute, densely hairy; bracteoles absent. Flowers: pedicels densely hairy; sepals free, persistent, margin entire, apex acute, densely hairy outside, glabrous inside, glandular hairs absent. Staminate inflorescences c. 3.5 $\mathrm{cm}$ long, nodes per branch 5-8, with 3-6 flowers per bract. Staminate flowers 4-5 $\mathrm{mm}$ diam.; pedicels $1-2 \mathrm{~mm}$ long; sepals 3 , ovoid, $3.2-3.3$ by $1.5-1.7 \mathrm{~mm}$; stamens $30-40$, filaments $2.5-4 \mathrm{~mm}$ long, basally connate, densely to sparsely hairy, thecae ovoid to ellipsoid, $0.1-0.2$ by $0.1-0.15 \mathrm{~mm}$, glabrous, connective widened; pistillode present. Pistillate inflorescences $4-10 \mathrm{~cm}$ long, nodes per branch 2-5, with 1 flower per bract. Pistillate flowers $2.4-2.7 \mathrm{~mm}$ diam.; pedicels $3-5 \mathrm{~mm}$ long; sepals 4 or 5 , narrowly triangular, $4.5-5$ by $1.8-2 \mathrm{~mm}$; ovary $1.8-2.3$ by $1.8-2 \mathrm{~mm}$, 3-locular, densely hairy, glandular hairs absent; style $1-2 \mathrm{~mm}$ long; stigmas $1.5-2$ by $0.5 \mathrm{~mm}$. Fruits capsules, $8-10$ by $13-16 \mathrm{~mm}$, pale green, opening septicidally-loculicidally, surface spiny, densely hairy, scatteredly covered with glandular hairs; spines 100-140, curved, $0.8-1$ by c. $0.6 \mathrm{~mm}$; wall c. $0.5 \mathrm{~mm}$ thick, sparsely hairy to glabrous inside; column $5-5.5$ by $6-8 \mathrm{~mm}$. Seeds \pm globose, $5-7$ by $6-7$ by $6-7 \mathrm{~mm}$, surface smooth, shiny, light brown.

Distribution - Thailand, Peninsular Malaysia.

Habitat \& Ecology - Locally in understorey of evergreen forest, near waterfalls and streams; on granite soil. Altitude: sea level up to $150 \mathrm{~m}$. Flowering and fruiting: February to May.

Vernacular names - Peninsular Malaysia: Tampay, kayu Roon Tiew (Temuan).

Uses - Poisonous fruits.

Note - Mallotus subcuneatus can easily be confused with M. montanus because of the hairs on the lower leaf surface, hairy filaments, seed shape and colour and their distribution in Thailand and Peninsular Malaysia. The main differences are found in domatia, leaf blade upper surface indument, bracts and fruits: domatia absent (present in M. montanus), upper surface of leaves glabrous (sparsely hairy in M. montanus), glandular teeth inconspicuous (conspicuous in M. montanus), bracts large, c. 3 by $0.7-1$ 
$\mathrm{mm}$ (small, $0.6-0.8$ by $0.4-0.6 \mathrm{~mm}$ in $M$. montanus); filaments basally connate (free in M. montanus), fruits with spines (absent in M. montanus), seeds large, $5-7$ by $6-7$ by $6-7 \mathrm{~mm}$, dark brown (small, $4.2-6.5$ by $4.2-6.5$ by $4.2-6.2 \mathrm{~mm}$, light brown in M. montanus).

\section{Mallotus subulatus Müll.Arg. - Map 16}

Mallotus subulatus Müll.Arg. (1865) 192; Prain (1913) 927. — Lectotype (selected here): Mann 260 (holo K; iso K), Equatorial Guinea, Bioko Island (Fernando-Po).

Shrubs, 1-5 m high, dioecious. Indumentum composed of simple and stellately-tufted hairs and light yellow glandular hairs. Stipules linear-triangular, $3.5-12$ by $0.3-1$ $\mathrm{mm}$, persistent to caducous, margin entire, apex acute, densely to scatteredly hairy outside, scatteredly hairy inside. Leaves opposite to rarely alternate; petiole $9.5-55$ by $1-2.5 \mathrm{~mm}$, densely to sparsely hairy; blade ovate to elliptic, $8.5-30$ by $3.5-16 \mathrm{~cm}$, length/width ratio 1.6-2.4, membranaceous to chartaceous, base cordate, truncate to obtuse, margin entire, sometimes shallowly dentate, with glandular teeth, apex acute to caudate, upper surface densely to sparsely hairy on midrib and nerves, extrafloral nectaries marginal in lower half, (1-) 3 or 4 per side, 1-7 mm from margin, elliptic to orbicular, $0.5-1$ by $0.2-0.5 \mathrm{~mm}$, lower surface sparsely hairy, scatteredly covered with glandular hairs, domatia sometimes present, venation triplinerved, nerves 5 or 6 per side, looping and closed near margin. Inflorescences single, erect; axes densely hairy; bracts narrowly to linear-triangular, $3.2-5$ by $0.3-1.3 \mathrm{~mm}$, margin entire, apex acute, densely to scatteredly hairy; bracteoles linear-triangular, $1-1.5$ by $0.2-0.3 \mathrm{~mm}$, persistent to caducous. Flowers: pedicels densely hairy; sepals persistent, margin entire, apex acute, densely hairy and sparsely covered with glandular hairs outside, sparsely

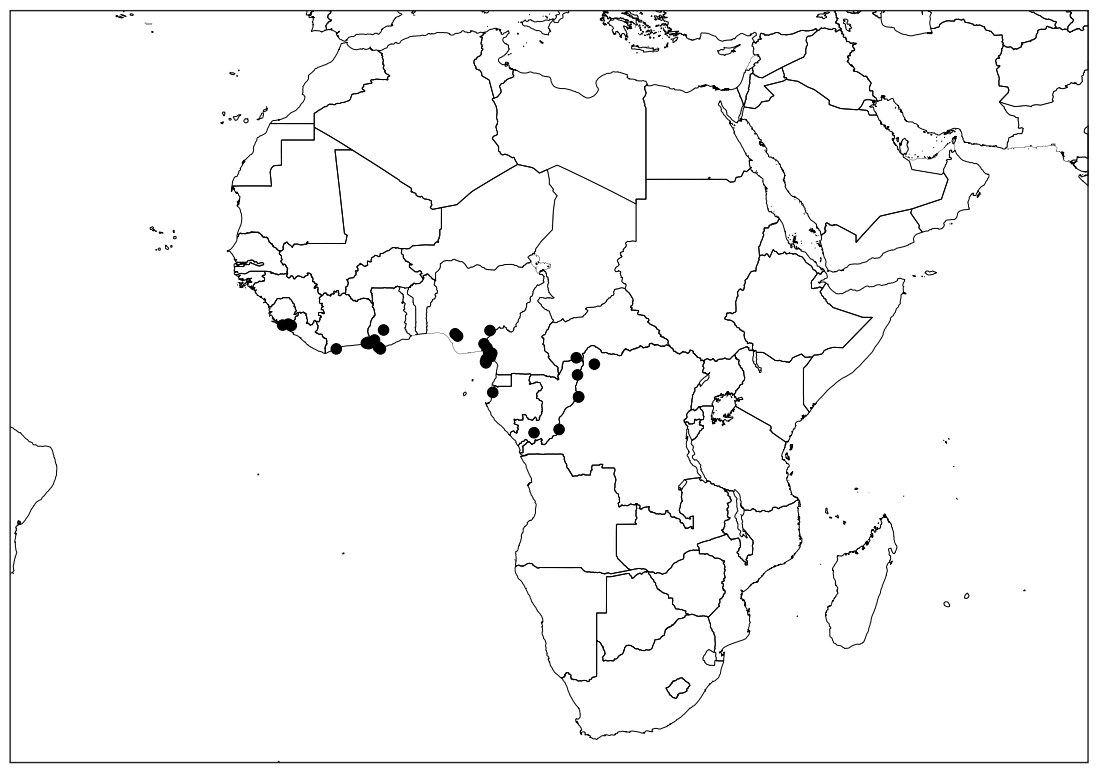

Map 16. Distribution of Mallotus subulatus Müll.Arg. 
hairy inside. Staminate inflorescences racemes, 2.7-13 cm long; peduncle 10-65 mm long; nodes per branch 7-14, with 3-10 flowers per bract. Staminate flowers 4-5 mm diam.; pedicels 5-6 mm long; sepals 3, ellipsoid, 3.2-4 by $1.9-2.2 \mathrm{~mm}$, free; stamens $42-50$, filaments $3-4 \mathrm{~mm}$ long, free, hairy, thecae ellipsoid, $0.5-0.7$ by $0.2-0.25$ $\mathrm{mm}$, hairy to glabrous, connective widened; pistillode present. Pistillate inflorescences racemes or umbel-like; $2-7.5 \mathrm{~cm}$ long; peduncle $15-40 \mathrm{~mm}$ long; nodes per branch 1-3, with (1-) 4-6 flowers per bract. Pistillate flowers 3.5-5 mm diam.; pedicels 4-12 mm long; calyx 3- or 4-lobed, lobes triangular, 3.5-4 by $1.4-1.6 \mathrm{~mm}$; ovary $1.7-2$ by 2-2.2 mm, 3- (or 4-)locular, densely hairy, scatteredly covered with glandular hairs; style c. $1 \mathrm{~mm}$ long; stigmas $3-4$ by $1.3-1.5 \mathrm{~mm}$. Fruits capsules, $10-11.5$ by $7-10$ $\mathrm{mm}$, opening septicidally-loculicidally, surface spiny, densely hairy, sparsely covered with glandular hairs; spines $300-400, \pm$ straight, $2-3$ by $0.1-0.2 \mathrm{~mm}$, densely hairy; wall $0.8-1 \mathrm{~mm}$ thick, sparsely hairy inside; column $2-5$ by $2-4 \mathrm{~mm}$. Seeds \pm globose, $5-5.3$ by $4-4.2$ by $4-4.2 \mathrm{~mm}$, surface smooth, shiny, light brown.

Distribution - Endemic to SW Africa (Sierra Leone to W Zaire).

Habitat \& Ecology - Locally common in secondary scrub and remnants of forest, along roadsides, between crops or in abandoned rice fields, in light shade or open areas. Altitude 20-240 m. Flowering and fruiting throughout the year.

Vernacular name - Nigeria: Fie ofume.

\section{Mallotus tiliifolius (Blume) Müll.Arg. - Map 17; Plate 2}

Mallotus tiliifolius (Blume) Müll.Arg. (1865) 190; in DC. (1866) 969. - Rottlera tiliifolia Blume (1826) 607 ('tiliaefolia'); Backer \& Bakh.f. (1964) 484; Airy Shaw (1972) 305; (1975) 170; (1980c) 171; (1981) 329; (1982) 30; (1983) 38; P.I. Forst. (1999) 489; Welzen et al. (2000) 110. - Rottlera blumei Decne. (1834) 486, nom. superfl. - Lectotype (selected here): Blume '1676' (holo L barcode L0294967; iso L barcode L0292539, BO), Java.

Halecus litorea Rumph. (1743) 196, t. 126, nom. inval.; Merr. (1917) 318. - Croton tiliifolius Lam. var. aromaticus auct. non (L.) Lam.: Lam. (1786) 206, quoad syn. Rumph.; Müll.Arg. in DC. (1866) 969 (see note). - Voucher: Rumphius plate 126. - Representative specimen: Robinson 367 (holo US; iso BM, L, NY), Moluccas, Ambon, Amboina.

Croton acuminatus Lam. (1786) 207 ('acuminatum'), non M. acuminatus Müll.Arg. (1865). Rottlera acuminata (Lam.) A. Juss. (1824) 33. - Type: Commerson s.n. (holo P-JU \# 16580 (IDC microfiche 6206)), Papua New Guinea, New Britain, Port Praslin.

Adelia papillaris Blanco (1845) 562. - Mallotus papillaris (Blanco) Merr. (1912a) 238; (1923) 434. - Mallotus zollingeri auct. non Müll.Arg.: Fern.-Vill. in Blanco (1880) 195. - Neotype (selected here): Merrill Species Blancoanae 50 (holo US; iso A, BM, F, GH, L, NY, P), Philippines, Batangas, San Pedro.

Mallotus playfairii Hemsl. in F.B. Forbes \& Hemsl. (1894) 441. - Type: Playfair 324 (holo K; photo in A), Taiwan, Ape Hill.

Croton enantiophyllus K. Schum. (1905) 296. - Type: Parkinson s.n. (holo B †; iso NSW barcode 604690, male specimen), Papua New Guinea, New Britain.

Mallotus palauensis Hosok. (1935) 25. - Type: Hosokawa 7284 (holo TAI; iso L), Republic of Palau, Babeldaob Island.

Mallotus ponapensis Hosok. (1935) 26. - Type: Hosokawa 6124 (holo TAI, iso TAI), Federated States of Micronesia, Pohnpei, Saba-tic.

Mallotus repandus auct. non (Rottler) Müll.Arg. (1865): K. Schum. \& Lauterb. (1901) 395. - Voucher: Naumann s.n. (G?, K?, LE? n.v.), Papua New Guinea, Neu-Hannover.

Shrubs to small trees, up to $15 \mathrm{~m}$ high, dioecious or rarely monoecious. Outer bark smooth and shallow longitudinal fissured. Indumentum tomentose, composed of simple, 
stellately-tufted and stellate hairs (on petiole and midrib of blade) and yellow to orange hairs (capitate glandular hairs are also present but not easy to detect). Stipules absent. Leaves opposite to rarely alternate, smelling of skunk when fresh; petiole $20-65$ by $1-2 \mathrm{~mm}$, densely hairy; blade broadly ovate to ovate, $4.5-21$ by $3.8-17.5$ $\mathrm{cm}$, length/width ratio 1-1.3, chartaceous, base cordate, truncate, rounded to cuneate, margin entire to denticulate, sometimes with glandular teeth, apex acute to caudate, upper surface sparsely hairy to scatteredly hairy, sometimes scatteredly covered with glandular hairs, extrafloral nectaries marginal from base (to apex), 2-8 per side, $0.3-2$ $\mathrm{mm}$ from margin, elliptic to orbicular, $0.3-0.8$ by $0.3-0.5 \mathrm{~mm}$, lower surface densely hairy, densely (to scatteredly) covered with glandular hairs, domatia absent, venation triplinerved, nerves 7-11 per side, looping and closed near margin or ending in margin. Inflorescences racemes, single, erect, unisexual or rarely bisexual; axes densely hairy; bracts triangular to narrowly triangular, $0.8-3$ by $0.5-1 \mathrm{~mm}$, margin entire, apex acute, densely hairy; bracteoles absent. Flowers: pedicels densely hairy; sepals margin entire, apex acute, densely hairy and with glandular hairs outside, sparsely hairy to glabrous inside. Staminate inflorescences 7-22 cm long; peduncle 30-40 mm long; nodes per branch 12-25, with 5-12 flowers per bract. Staminate flowers $2.8-5.5 \mathrm{~mm}$ diam.; pedicels $2-4 \mathrm{~mm}$ long; sepals 4 or 5 , ellipsoid to ovoid, $2.5-3$ by $0.9-1.5 \mathrm{~mm}$; stamens $45-70$, glabrous, filaments $0.5-2.3 \mathrm{~mm}$ long, free (rarely aggregated into a cluster), thecae ellipsoid, $0.2-0.3$ by $0.2-0.25 \mathrm{~mm}$, connective widened; pistillode present. Pistillate inflorescences 5-15 cm long; peduncle 10-40 mm long; nodes per branch 9-16, with 1 flower per bract. Pistillate flowers $2-4 \mathrm{~mm}$ diam.; pedicels $1.7-2.5 \mathrm{~mm}$ long; calyx $4-6$-lobed, lobes narrowly triangular, $2-2.3$ by $0.5-0.7 \mathrm{~mm}$; staminodes rarely present (usually persistent in fruits); ovary $1.3-1.5$ by $1.2-1.4 \mathrm{~mm}$,

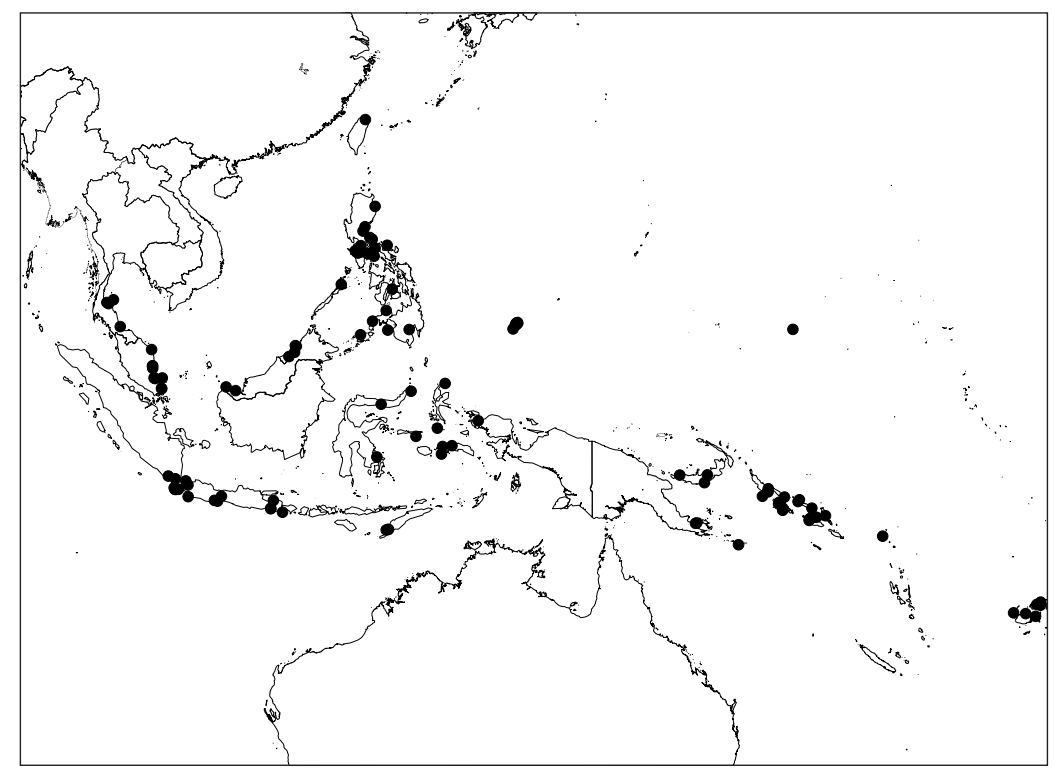

Map 17. Distribution of Mallotus tiliifolius (Blume) Müll.Arg. 
3- (or 4-)locular, densely hairy, sparsely covered with glandular hairs; style 0.4-0.7 mm long; stigmas $2.5-4.3$ by $0.8-1 \mathrm{~mm}$. Fruits capsules, $5-15$ by $7-15 \mathrm{~mm}$, opening septicidally-loculicidally, surface spiny, densely hairy, sparsely covered with glandular hairs; spines $15-60$, straight, $0.5-2$ by $0.2-0.3 \mathrm{~mm}$, densely hairy; wall $0.8-1 \mathrm{~mm}$ thick, sparsely hairy inside; column $4-6$ by $3-4 \mathrm{~mm}$. Seeds globose, $5-7$ by $5-7$ by 4.7-5 mm, surface smooth, dull, light brown-orange.

Distribution - From Thailand to Taiwan, throughout Peninsular Malaysia, Malesia, Republic of Palau, and Federated States of Micronesia to the W Pacific (Solomon Islands, Fiji).

Habitat \& Ecology - Primary forest, understorey of evergreen to deciduous forest, coastal or disturbed riparian vegetations; on edges of mangroves, swampy areas, sandy areas behind the beach, along forest margins, trails, steep valley slopes, lake and sea shores, the end of tidal marsh; in wet terrains. Altitude: sea level up to $350 \mathrm{~m}$. Flowering and fruiting throughout the year.

Vernacular names - Java: Djasilin, ring rin, waru lot. Brunei: Tapian batu. Malaysia/Sabah: Tuka tuak laut. Philippines: Anamú saláurid, gapas gapas. Moluccas: Warwar. Sulawesi: Lendé. New Guinea: Maimeireh (Gabobora); Idirur (Onjob). Solomon Islands: Airafu, airahu, alabuis kwo, albuis, hezoro, ma'aqa, sautalau, s. susui, j. arifanata, ena-cui, grapusi-nimarau, r. mauriasi (Kwara'ae). Caroline Islands: Cherin. Fiji: Banglut, daporan, pijalan (Bajau); nganga (Ra).

Note - Lamarck (1786) described Croton tiliifolius with a variety $\beta$ aromaticus. As it turns out Croton tiliifolius consists of three taxa. The typical variety is based on a Commerson collection from Mauritius, which is a true Croton (see Coode (1982: 44)). The variety $\beta$ consists of two parts, based on references to Linnaeus: Fl. Zeylanica (1747) and Rumphius' Herb. Amboin. 3 (1743). The first part, "Croton foliis cordatis scabris subserratis petiolatis caule arboreo" from Sri Lanka, was used by Linnaeus in 1753 for his Croton aromaticus, which is also still regarded as a Croton (Philcox, 1997: 101). The second part, which has caused the most confusion, is the Halecus litorea of Rumphius from Ambon. It is a Mallotus as plate 126 in Rumphius' book shows. This was also observed by Merrill (1917: 318), who gave a brief survey on the identification of the Rumphian species.

Another cause of the confusion is that Blume described a Rottlera tiliifolia (1826) which has been equated with Rumphius' species and probably because of the similarity of the epithet with Lamarck's species. An example of this is found in Müller Argoviensis (1865) where he made the combination Mallotus tiliifolius. The first reference given is to the Lamarck's name, the second to the one of Blume and the third to Rottlera acuminata A. Juss. (from Timor and a synonym of Blume's combination). It would therefore appear that the combination is based on the Mauritian name. However, this is not so, as is evident from the treatment in 1866, where Müller clearly distinguished between Croton tiliifolius Lam. (p. 587), Croton aromaticum L. (p. 588) and Mallotus tiliifolius Müll.Arg. (p. 969). In the publications of 1865 and 1866 the last taxon has exclusively Southeast Asian and Chinese collections and what obviously happened was that the letter $\beta$ after Croton tiliifolius Lam. was accidentally omitted in 1865 .

The distinction between Croton tiliifolius and Rottlera tiliifolius was also made by Decaisne (1834) who erroneously renamed Blume's taxon to Rottlera blumei. Blume's epithet tiliifolius is in fact younger (1826) than the epithet acuminatus (De Jussieu's 
name is based on Croton acuminatum Lam., 1786). However, the combination Mallotus acuminatus was already in use (also by Müller Argoviensis) and, therefore, the epithet tiliifolius is then the oldest available. The combination proposed is based on that of Blume and the correct name for the taxon therefore must be Mallotus tiliifolius (Blume, and not Lam.!) Müll.Arg.

\section{Mallotus trinervius (K. Schum. \& Lauterb.) Pax \& K. Hoffm. - Map 2}

Mallotus trinervius (K. Schum. \& Lauterb.) Pax \& K. Hoffm. (1914) 156. - Syndyophyllum trinervium K. Schum. \& Lauterb. (1901) 405. - Coelodiscus trinervius (K. Schum. \& Lauterb.) Pax (1910) 481; Pax \& K. Hoffm. (1911) 105. - Type: Tappenbeck 84 (holo B? n.v.), New Guinea, Kaiser-Wilhelms-Land, Ramufluss.

Small trees, 6-19 m high; bole up to $11 \mathrm{~m}$ high, dbh $18-40 \mathrm{~cm}$, dioecious, crown up to $8 \mathrm{~m}$ high. Outer bark shallowly longitudinally fissured. Indumentum composed of simple, stellately-tufted and stellate hairs (on petiole and midrib of blade) and yellow glandular hairs. Stipules triangular to narrowly triangular, $1.8-2$ by $0.4-0.6 \mathrm{~mm}$, early caducous, margin entire, apex acute, densely hairy outside, sparsely hairy inside. Leaves opposite; petiole $30-90$ by $1.8-2.5 \mathrm{~mm}$, sparsely hairy; blade ovate to elliptic, $9-26$ by $6-15.5 \mathrm{~cm}$, length/width ratio $1.4-1.8$, chartaceous, base rounded, obtuse to shortly attenuate, margin entire, apex obtuse, acute to acuminate, upper surface sparsely to scatteredly hairy on midrib, extrafloral nectaries marginal from base to apex, 7-25 per side, $0.5-6 \mathrm{~mm}$ from margin, elliptic to orbicular, $0.2-0.6$ by $0.3-0.6 \mathrm{~mm}$, lower surface densely (see note) to sparsely hairy, glabrescent, sometimes scatteredly covered with glandular hairs, domatia absent, venation triplinerved, nerves 5-7 per side, looping and closed near margin. Staminate inflorescences racemes, 9-17 cm long, single, erect; axes densely hairy; peduncle $8-29$ by $1-1.5 \mathrm{~mm}$; nodes per branch $16-20$, with $5-7$ flowers per bract; bracts triangular, $0.9-1.1$ by $0.7-0.9 \mathrm{~mm}$, margin entire, apex acute, densely hairy outside, glabrous inside, early caducous; bracteoles absent. Staminate flowers 5-6 mm diam.; pedicels $2-3.5 \mathrm{~mm}$, densely hairy; calyx 3-5-lobed, lobes ovate to elliptic, $3.8-4.8$ by $1.5-2.5 \mathrm{~mm}$, persistent, margin entire, apex acute, densely hairy outside, glabrous inside, without glandular hairs; stamens 100-120, glabrous, filaments $2.5-5 \mathrm{~mm}$ long, free, thecae ellipsoid, $0.2-0.3$ by $0.2-0.25 \mathrm{~mm}$, connective widened; pistillode present. Pistillate inflorescences and pistillate flowers unknown; infructescences $4-6 \mathrm{~cm}$ long; peduncle $3-55$ by $1-2.2 \mathrm{~mm}$ long; nodes per branch $7-19$, with 1 flower per bract; sepals $4-6$, narrowly triangular, $2.4-3$ by $0.8-1.1 \mathrm{~mm}$; ovary 2 - or 3- (or 4-)locular, densely hairy. Fruits tardily dehiscent capsules, 17-22.5 by 24-29 $\mathrm{mm}$, opening loculicidally, surface smooth, densely hairy, glandular hairs absent; wall $3-8 \mathrm{~mm}$ thick, sparsely hairy to glabrous inside; column $10-15$ by $2.8-3 \mathrm{~mm}$. Seeds \pm globose, $9-10$ by $8.5-9.5$ by $7-8.5 \mathrm{~mm}$, surface rugose, dull, whitish cream.

Distribution - Endemic to New Guinea.

Habitat \& Ecology - In primary or secondary forest, occasionally in flooded rainforests of river plains, in swamp-forests or swamp-grasslands; on alluvial or peaty soil. Altitude: sea level up to $1400 \mathrm{~m}$ (Airy Shaw 1980c: 172, mentions 810-3450 m).

Note - See also note 2 for M. didymochryseus. 


\section{NOTES ON UNIDENTIFIED SPECIMENS}

\section{Brass 1333, New Guinea, Murua River.}

The specimen looks like $M$. attenuatus in the presence of inconspicuous extrafloral nectaries and absence of glandular hairs. However, the leaves are triplinerved, while in M.attenuatus they are pinnate.

2. Brass 8844 , New Guinea, Tabati, Jatutefa Bay.

The specimen looks like the Australian species M. megadontus in the appearance of the leaves and fruits.

3. Turner 98, New Guinea, Eastern District, Fife bay.

The specimen looks like M. lauterbachianus in the appearance of the leaves. However, the male inflorescences are umbel-like, while in M. lauterbachianus they are racemes.

4. Widjaja et al. 6697, 7079, New Guinea, Irian Jaya, Kab. Yapen.

The specimens share the presence of extrafloral nectaries along the midrib and of few spines on the fruits with M. macularis. However, they are much less hairy compared to M. macularis.

\section{DUBIOUS SPECIES}

Axenfeldia intermedia Baill. (1858) 419. - Mallotus intermedius (Baill.) N.P. Balakr. (1968) 245. - Syntypes: Unknown s.n. (P n.v.), India = cf. M. resinosus.

Mallotus bracteatus Hook.f. (1887) 436. - Type: Scortechini s.n. (?), Peninsular Malaysia, Perak.

Mallotus buettneri Pax (1895) 89. - Type: Büttner 272 (holo B? n.v.), Gabon, Munda, Sibange $=$ cf. M. subulatus.

Mallotus lawii Müll.Arg. (1865) 192. - Type: Stocks \& Law 'in Hook.f. \& Thoms. Hb. Ind. Or. Claoxyl. 6' (holo G-DC (IDC microfiche 800); iso L, NY), India, Malabar $=$ cf. M. resinosus.

Mallotus sanguirensis Pax \& K. Hoffm. (1919) 18. - Type: Warburg 16634 (B†), Sulawesi, Sangihe $=$ cf. M. resinosus.

Mallotus smilaciformis Gage (1922) 242. - Syntypes: King's collector 3134 (?); 3145 (?), Malay Peninsula, Perak, Lalema = cf. M. korthalsii.

Mallotus subramanyamii J.L. Ellis (1983) 199. - Mallotus resinosus (Blanco) Merr. var. subramanyamii (J.L. Ellis) Chakrab. (1985) 704. - Type: J.L. Ellis 34963 (holo CAL n.v.; iso MH n.v.), India, Tamil Nadu, Nilgiri Hills, Moolakadu, Ouchterlong Valley $=$ cf. M. resinosus.

Rottlera aureopunctata Dalzell (1851) 122; Dalzell \& A. Gibson (1861) 230. - Mallotus aureo-punctatus (Dalzell) Müll.Arg. in DC. (1866) 973. - Syntypes: Unknown s.n. (CAL?, DD?, K?, W?), India, shady jungles in the Ghauts (Ghats), hilly parts, Concan; Meera Hills. 'Noticed in Graham's list under 1334' = cf. M. resinosus. 


\section{EXCLUDED SPECIES}

Mallotus brevipes Merr. (1914) 487. - Type: R.S. Williams 2968 (holo PNH†; iso NY, 2 sheets), Philippines, SE Mindanao, Davao Prov. = Lasiococca brevipes (Merr.) Welzen \& S.E.C. Sierra (Van Welzen \& Sierra, 2005).

\section{ACKNOWLEDGEMENTS}

The directors of the following institutes are thanked for loans of their material: A, AAU, AD, B, BM, BP, BR, BRI, C, CAHUP, CAL, CANB, CGE, DBN, DD, DPU, DS, E, F, FI, G, GH, GJO, H, HAL, HK, IBSC, JE, K, KEP, KIEL, L, LD, LE, LIV, M, MA, MANCH, MEL, MICH, MO, MSB, NSW, NY, OXF, P, PDA, PH, PR, S, SAN, SBT, SING, SYS, TAI, TCD, TI, TU, U, UC, US, W, WAG, WRSL, ZT. Special thanks are due to J.F. Veldkamp for his valuable help concerning nomenclatural problems; Ž. Fišer and P. Baas for their comments on leaf anatomy; R.W.J.M. van der Ham for his comments on pollen; A. Walsmit Sachs, M. Nakajima, O. Kirdkaew for the drawings; N. Raes for the maps; N. Sol and G. Thijsse for the administration of the collections, L. Frankova and D.N.F. Kiehl $\dagger$ for help with the literature; J.M. de Wolf, B.J. van Heuven for technical support; J.B. Mols and J.W.F. Slik for helpful discussions; the reviewers for their comments on the manuscript.

\section{REFERENCES}

Airy Shaw, H.K. 1966. Notes on Malaysian Euphorbiaceae. Kew Bull. 20: 38-45.

Airy Shaw, H.K. 1968. Malesian and other Asiatic Euphorbiaceae. Kew Bull. 21: 379-400.

Airy Shaw, H.K. 1971. New or noteworthy species of Mallotus Lour. Kew Bull. 25: 526-528.

Airy Shaw, H.K. 1972. The Euphorbiaceae of Siam. Kew Bull. 26: 292-308.

Airy Shaw, H.K. 1975. The Euphorbiaceae of Borneo. Kew Bull., Addit. Ser. 4: 160-174.

Airy Shaw, H.K. 1977. Additions and corrections to the Euphorbiaceae of Siam. Kew Bull. 32: 69-83.

Airy Shaw, H.K. 1978a. Notes on Malesian and other Asiatic Euphorbiaceae. CXCIX. New or noteworthy species of Mallotus Lour. Kew Bull. 32: 400-407.

Airy Shaw, H.K. 1978b. Notes on Malesian and other Asiatic Euphorbiaceae. CCXVI. Mallotus Lour. Kew Bull. 33: 62-66.

Airy Shaw, H.K. 1980a. New Euphorbiaceae from New Guinea. Kew Bull. 34: 597-598.

Airy Shaw, H.K. 1980b. A partial synopsis of the Euphorbiaceae-Platylobeae of Australia (excluding Phyllanthus, Euphorbia and Calycopetalus). Kew Bull 35: 649-657.

Airy Shaw, H.K. 1980c. The Euphorbiaceae of New Guinea. Kew Bull., Addit. Ser. 8: 162-172, 225.

Airy Shaw, H.K. 1980d. New or noteworthy Australian Euphorbiaceae - II. Muelleria 4: 207-235. Airy Shaw, H.K. 1981. The Euphorbiaceae of Sumatra. Kew Bull. 36: 239-374.

Airy Shaw, H.K. 1982. The Euphorbiaceae of Central Malesia (Celebes, Moluccas, Lesser Sunda Is.). Kew Bull. 37: 28-30.

Airy Shaw, H.K. 1983. An alphabetical enumeration of the Euphorbiaceae of the Philippine islands. Royal Botanic Gardens, Kew.

Backer, C.A. \& R.C. Bakhuizen van den Brink Jr. 1964. Flora of Java 1. Noordhoff, Groningen.

Baillon, H.E. 1858. Étude générale du groupe des Euphorbiacées. Masson, Paris.

Baillon, H.E. 1861. Species Euphorbiacearum. Euphorbiacées africaines. Première partie, Afrique occidentale (Sénégambie, îles du Cap Vert, Nigritie et Guinée). Adansonia 1: 59-69.

Baillon, H.E. 1866. Species Euphorbiacearum. Euphorbiacées Australiennes. Adansonia 6: 313317.

Baillon, H.E. 1891. Liste des plantes de Madagascar. Bull. Mens. Soc. Linn. Paris 2: 977-978.

Balakrishnan, N.P. 1968. Studies in Indian Euphorbiaceae III: Miscellaneous notes. Bull. Bot. Surv. India 10: 245. 
Balakrishnan, N.P \& T. Chakrabarty. 1991. Mallotus resinosus (Blanco) Merr. (Euphorbiaceae) and its allies. Rheedea 1: 36-39.

Beddome, R.H. 1873. Flora sylvatica 2. Adelphi Press, Madras.

Beille, L. 1908. Euphorbiaceae. In: A. Chevalier, Novitates florae africanae. Bull. Soc. Bot. France 55, Mém. 8: 76.

Blanco, F.M. 1845. Flora de Filipinas (ed. 2). Sanchez, Manila.

Blanco, F.M. 1880. Flora de Filipinas (ed. 3). Plana y Ca., Manila.

Blume, C.L. 1826. Bijdragen tot de flora van Nederlandsch-Indië 11: 485-730. Lands Drukkerij, Batavia.

Blüthgen, N., A. Metzner \& D. Ruf. 2006. Food plant selection by stick insects (Phasmida) in a Bornean rain forest. J. Trop. Ecol. 22: 35-40.

Bollendorff, S.M., P.C. van Welzen \& J.W.F. Slik. 2000. A taxonomic revision of Mallotus section Polyadenii (Euphorbiaceae). Blumea 45: 319-340.

Chakrabarty, T. 1985. A note on Mallotus subramanyamii Ellis (Euphorbiaceae). J. Econ. Taxon. Bot. 6: 704.

Coode, M.J.E. 1982. Euphorbiaceae. In: Flora des Mascareignes 160: 44-46. The Sugar Industries Research Institute, Mauritius; l'Office de la Recherche Scientifique et Technique outre-mer, Paris; The Royal Botanical Gardens, Kew.

Corlett, R.T. 2001. Pollination in a degraded tropical landscape: a Hong Kong case study. J. Trop. Ecol. 17: 155-161.

Dalzell, N.A. 1851. Contributions to the botany of western India. Hooker's J. Bot. Kew Gard. Misc. 3: $122-123$.

Dalzell, N.A. \& A. Gibson. 1861. The Bombay flora: 230. Education Society's Press, Bombay.

De Candolle, A. 1866. Prodromus 15, 2. Masson \& Fili, Paris.

De Jussieu, A.H.L. 1824. De Euphorbiacearum generibus. Didot Jr., Paris.

De Lamarck, J.B.A.P. 1786. Encyclopédie méthodique. Botanique. 2. Panckoucke, Paris.

Decaisne, J. 1834. Rottlera blumei. Nouv. Ann. Mus. Hist. Nat. 3, 3: 486.

Dinerstein, E. \& C.M. Wemmer. 1988. Fruits rhinoceros eat: Dispersal of Trewia nudiflora (Euphorbiaceae) in lowland Nepal. Ecology 69: 1768-1774.

Domin, K. 1927. Mallotus Lour. Biblioth. Bot. Band 22, Heft 89, Abt. 3: 887-889.

Dwivedi, A. 1994. Seed development in Trewia nudiflora Linn. Geophytology 24: 101-106.

Ellis, J.L. 1983. Mallotus subramanyamii Ellis (Euphorbiaceae) - A new species from the western slopes of Nilgris, Peninsular India. Bull. Bot. Surv. India 25: 199-201.

Esser, H.-J. 2003. Fruit characters in Malesian Euphorbiaceae. Telopea 10: 169-177.

Forbes, F.B. \& W.B. Hemsley. 1894. An enumeration of all the plants known from China Proper, Formosa, Hainan, Corea, the Luchu archipelago, and the Island of Hongkong, together with their distribution and synonymy. J. Linn. Soc., Bot. 26: 397-456.

Forster, P.I. 1999. A taxonomic revision of Mallotus Lour. (Euphorbiaceae) in Australia. Austrobaileya 5: 457-497.

Gage, A.T. 1922. Euphorbiaceae novae e Peninsula Malayana. Rec. Bot. Surv. India 9: 240-243.

Gagnepain, F. 1923. Euphorbiacées nouvelles. Notul. Syst. (Paris) 4: 49-54.

Gagnepain, F. 1925. Euphorbiaceae. In: M.H. Lecomte (ed.), Flore générale de l'Indo-Chine 5: 229_ 673. Masson \& Cie, Paris.

Geiseler, E.F. 1807. Crotonis monographiam, speciminis loco inauguralis, ut doctoris medici gradum in alma fridericiana adipiscatur. Grunert, Halle.

Govaerts, R., D.G. Frodin \& A. Radcliffe-Smith. 2000. World checklist and bibliography of Euphorbiaceae 3 \& 4. Royal Botanic Gardens, Kew.

Hasskarl, J.C. 1842. Plantarum genera et species novae aut reformatae javenses. Flora 25: 21-114.

Hewson, H.J. 1998. Plant Indumentum. A handbook of terminology. Australian Flora \& Fauna Series 9. Canberra: Australian Government Publishing Series.

Hooker, J.D. 1887. Flora of British India 5. Reeve \& Co., London.

Hooker, J.D. \& G. Bentham. 1849. Flora Nigritiana. In: W.J. Hooker (ed.), Niger Flora: 199-577. Hippolyte Bailliere, London.

Hosokawa, T. 1935. Materials of the botanical research towards the flora of Micronesia. Trans. Nat. Hist. Soc. Formosa 25: 17-29. 
Hwang, S.M. 1985. New material of Mallotus L. from China. Acta Phytotax. Sin. 23: 293-301.

Juss. - see De Jussieu

Kress, W.J., R.A. DeFilipps, E. Farr \& D. Yin Yin Kyi. 2003. A checklist of the trees, shrubs, herbs, and climbers of Myanmar (rev. ed. 4). National Museum of Natural History, Washington, DC.

Kulju, K.K.M., S.E.C. Sierra, S.G.A. Draisma, R. Samuel \& P.C. van Welzen. In press. Phylogeny of Macaranga, Mallotus, and related genera (Euphorbiaceae s.s.): insights from plastid and nuclear sequence data. Amer. J. Bot.

Kulju, K.K.M., S.E.C. Sierra \& P.C. van Welzen. 2007. Re-shaping Mallotus [Part 2]: Inclusion of Neotrewia, Octospermum and Trewia to Mallotus s.s. (Euphorbiaceae s.s.). Blumea 52: 115-136.

Kuntze, O. 1891. Revisio Generum Plantarum 2. Arthur Felix, Leipzig.

Kurz, S. 1873. New Burmese plants. J. Asiat. Soc. Bengal 42: 227-254.

Kurz, S. 1877. Euphorbiaceae. Forest Fl. Burma 2: 378-397.

Lam. - see De Lamarck

Lauener, L.A. 1983. Catalogue of the names published by Hector Léveillé: XVI. Notes Roy. Bot. Gard. Edinburgh 40: 475-505.

Léveillé, A.A.H. 1911. Decades plantarum novarum. LIV-LVIII. Repert. Spec. Nov. Regni Veg. 9: 321-330.

Léveillé, A.A.H. 1914. Flore du Kouy-Tchéouchéou. Léveillé, Le Mans.

Linnaeus, C. 1747. Flora Zeylanica. Sumtu \& literis Laurentii Salvii, Stockholm.

Linnaeus, C. 1753. Species plantarum 2. Laurentii Salvii, Stockholm.

Lugt, Ch.B. 2003. Mallotus. In: R.H.M.J. Lemmens \& N. Bunyapraphatsara (ed.), Plant Resources of South-East Asia 12, 3. Medicinal and poisonous plants: 287-291. Backhuys Publishers, Leiden.

Merrill, E.D. 1912a. Nomenclatural and systematic notes on the flora of Manila. Philipp. J. Sci., Bot. 7: 227-240.

Merrill, E.D. 1912b. Notes on Philippine Euphorbiaceae. Philipp. J. Sci., Bot. 7: 379-410.

Merrill, E.D. 1914. Notes on Philippine Euphorbiaceae, II. Philipp. J. Sci., Bot. 9: 461-493.

Merrill, E.D. 1917. An interpretation of Rumphius' Herbarium Amboinense. Bur. Sci. Publ. 9: 313 329.

Merrill, E.D. 1918. Species Blancoanae: 221-223. Bureau of Printing, Manila.

Merrill, E.D. 1923. An enumeration of Philippine flowering plants 2: 432-437. Bureau of Printing, Manila.

Miquel, F.A.W. 1861. Flora van Nederlandsch Indië, Eerste Bijvoegsel, Sumatra. Van der Post, Amsterdam.

Moog, U., B. Fiala, W. Federle \& U. Maschwitz. 2002. Thrips pollination of the dioecious ant plant Macaranga hullettii (Euphorbiaceae) in Southeast Asia. Amer. J. Bot. 89: 50-59.

Moritzi, A. 1846. Systematisches Verzeichniss der von H. Zollinger in der Jahren 1842-1844 auf Java gesammelten Pflanzen. Zepfel, Solothurn.

Mueller, F. 1858. Euphorbiaceae. Fragmenta Phytographie Australiae 1: 31-33. Government Printer, Melbourne.

Müller Argoviensis, J. 1865. Euphorbiaceae. Linnaea 34: 184-197.

Müller Argoviensis, J. 1866. Euphorbiaceae tribus Acalypheae. In: A. de Candolle (ed.), Prodr. 15, 2: 1-1286. Masson \& Fili, Paris.

Nowicke, J.W. \& M. Takahashi. 2002. Pollen morphology, exine structure and systematics of Acalyphoideae (Euphorbiaceae), part 4: tribes Acalypheae pro parte (Erythrococca, Claoxylon, Claoxylopsis, Mareya, Mareyopsis, Discoclaoxylon, Micrococca, Amyrea, Lobanilia, Mallotus, Deuteromallotus, Cordemoya, Cococceras, Trewia, Neotrewia, Rockinghamia, Octospermum, Acalypha, Lasiococca, Spathiostemon, Homonoia), Plukenetieae (Haematostemon, Astrococcus, Angostyles, Romanoa, Eleutherostigma, Plukenetia, Vigia, Cnesmone, Megistostigma, Sphaerostylis, Tragiella, Platygyna, Tragia, Acidoton, Pachystylidium, Dalechampia), Omphaleae (Omphalea), and discussion and summary of the complete subfamily. Rev. Palaeobot. Palynol. 121: 231-336.

Pax, F. 1895. Euphorbiaceae africanae II. Bot. Jahrb. Syst. 19: 76-127. 
Pax, F. 1897. Euphorbiaceae africanae III. Bot. Jahrb. Syst. 23: 518-536.

Pax, F. 1910. Zwei Coelodiscus-Arten aus Neu-Guinea. Feddes Repert. Spec. Nov. Regni Veg. 8: 481-482.

Pax, F. \& K. Hoffmann. 1911. Euphorbiaceae-Cluytiae. In: A. Engler (ed.), Pflanzenreich IV.147. iii: 105. Engelmann, Leipzig.

Pax, F. \& K. Hoffmann. 1914. Euphorbiaceae-Acalyphae-Mercurialinae. In: A. Engler (ed.), Pflanzenreich IV.147.vii: 145-212, 394-397. Engelmann, Leipzig.

Pax, F. \& K. Hoffmann. 1919. Euphorbiaceae-Additamentum VI. In: A. Engler (ed.), Pflanzenreich IV.147.xiv: 17-19. Engelmann, Leipzig.

Philcox, D. 1997.Euphorbiaceae. In: M.D. Dassanayake \& W.D. Clayton (eds.), A revised handbook to the flora of Ceylon 11: 80-166. Balkema, Rotterdam.

Prain, D. 1913.Euphorbiaceae. In: W.T. Thiselton-Dyer (ed.), Flora of Tropical Africa 6, 1:927-930. Reeve \& Co., London.

Radcliffe-Smith, A. 1974. Notes on African Euphorbiaceae: V. Kew Bull. 29: 436-437.

Radcliffe-Smith, A. 1984. Notes on African Euphorbiaceae: XIV. Kew Bull. 39: 789-790.

Radcliffe-Smith, A. 1985. Notes on African Euphorbiaceae: XVI. Kew Bull. 40: 658.

Radcliffe-Smith, A. 2001. Genera Euphorbiacearum. Royal Botanic Gardens, Kew.

Reichenbach, H.G. \& H. Zollinger. 1856. Over soorten van Rottlera. Acta Soc. Regiae Sci. IndoNeerl. 1: 1-32.

Ridley, H.N. 1911. The Flora of Lower Siam. J. Straits Branch Roy. Asiat. Soc. 59: 181.

Ridley, H.N. 1924. The Flora of the Malay Peninsula 3. Reeve \& Co., Ashford, Kent.

Rumphius, G.E. 1743. Herbarium Amboinense 3: 196, t. 126. Changuion, Catuffe \& Uytwerf, Amsterdam.

Sato, S. \& A. Sakai. 2005. Birds as dispersers of Mallotus japonicus in a coniferous plantation. Jap. J. Ornithol. 54: 23-28.

Scheffer, R.H.C.C. 1869. Observationes de Quibusdam Euphorbiaceis Archipelagi Indici. Ann. Mus. Bot. Lugduno-Batavum 4: 119-127.

Schumacher, F.C. 1827. Beskrivelse af Guineiske planter som ere fundne af danske Botanikere, preprint, second part: 1-236.

Schumann, K. 1905. Croton enantiophyllus K. Sch. In: K. Schuman \& K. Lauterbach, Nachträge zur Flora der Deutschen Schutzgebiete in der Südsee: 296. Borntraeger, Leipzig.

Schumann, K. \& K. Lauterbach. 1901. Die Flora der Deutschen Schutzgebiete in der Südsee. Borntraeger, Leipzig.

Sierra, S.E.C., M. Aparicio, K.K.M. Kulju,Ž. Fišer,P.C. van Welzen \& R.W.J.M. van der Ham. 2006. Re-shaping Mallotus [Part 1]: Expanded circumscription and revision of the genus Cordemoya (Euphorbiaceae). Blumea 51: 519-540.

Sierra, S.E.C. \& P.C. van Welzen. 2005. A taxonomic revision of Mallotus section Mallotus (Euphorbiaceae) in Malesia. Blumea 50: 249-274.

Sierra, S.E.C. \& P.C. van Welzen. 2006. Mallotus kongkandae (Euphorbiaceae): New record for China. Blumea 51: 365-366.

Sierra, S.E.C., P.C. van Welzen \& J.W.F. Slik. 2005. A taxonomic revision of Mallotus section Philippinenses (former section Rottlera-Euphorbiaceae) in Malesia and Thailand. Blumea 50: 221-248.

Slik, J.W.F. 2005. Assessing tropical lowland forest disturbance using plant morphological and ecological attributes. Forest Ecology and Management 205: 241-250.

Slik, J.W.F., P.J.A. Keßler \& P.C. van Welzen. 2003. Macaranga and Mallotus species (Euphorbiaceae) as indicators for disturbance in the mixed lowland dipterocarp forest of East Kalimantan (Indonesia). Ecological Indicators 2, 4: 311-324.

Slik, J.W.F., Priyono \& P.C. van Welzen. 2000. Key to the Macaranga Thou. and Mallotus Lour. species (Euphorbiaceae) of East Kalimantan, Indonesia. Gard. Bull. Singapore 52: 11-87.

Slik, J.W.F. \& P.C. van Welzen. 2001a. A taxonomic revision of Mallotus sections Hancea and Stylanthus (Euphorbiaceae). Blumea 46: 3-66.

Slik, J.W.F. \& P.C. van Welzen. 2001b. A phylogeny of Mallotus (Euphorbiaceae) based on morphology: Indications for a pioneer origin of Macaranga. Syst. Bot. 26: 786-796.

Smitinand, T. 2001. Thai plant names, rev. ed. The Forest Herbarium, Bangkok. 
So, M.L. 2004. The occurrence of extrafloral nectaries in Hong Kong plants. Bot. Bull. Acad. Sin. 45: 237-245.

Thin, N.N. \& T.V. On. 1998. Mallotus. In: M.S.M. Sosef, L.T. Hong \& S. Prawirohatmodjo (eds.), Plant Resources of South-East Asia 5, 3. Timber trees. Lesser-known timbers: 347-350. Backhuys Publishers, Leiden.

Thwaites, G.H.K. 1861. Enumeratio plantarum zeylanae. Dulau \& Co., London.

Van Welzen, P.C. \& K. Chayamarit. 2001. Two new Mallotus and two new Sauropus species (Euphorbiaceae) endemic to Thailand. Kew Bull. 56: 649-656.

Van Welzen, P.C., R.M.A.P. Haegens, J.W.F. Slik, S.M. Bollendorff, S. Dressler \& H.-J. Esser. 2000. Checklist of the genera of Thai Euphorbiaceae. Thai Forest Bull., Bot. 28: 93-111.

Van Welzen, P.C., R.W.J.M. van der Ham \& K.K.M. Kulju. 2004. Mallotus glomerulatus (Euphorbiaceae), a new species: its description, pollen, and phylogenetic position. Thai Forest Bull., Bot. 32: 173-178.

Van Welzen, P.C. \& S.E.C. Sierra. 2005. Lasiococca brevipes (Euphorbiaceae): A new combination. Blumea 50: 275-277.

Van Welzen, P.C. \& S.E.C. Sierra. 2006. The Mallotus wrayi complex (Euphorbiaceae). Blumea 51: 373-388.

Van Welzen, P.C., S.E.C. Sierra, M.J.H. Gebraad \& K.K.M. Kulju. 2006. The distinction of five Mallotus species formerly in section Hancea (Euhporbiaceae). Blumea 51: 367-372.

Wallich, N. 1845?. A numerical list of dried specimens of plants in the East India Company's museum. Wallich, London.

Webster, G.L. 1994. Classification of the Euphorbiaceae. Ann. Missouri Bot. Gard. 81: 3-32.

Welzen - see Van Welzen, P.C.

Whitmore, T.C. 1973. Tree Flora of Malaya 2. Longman, London.

Wight, R. 1852. Icones plantarum Indiae Orientalis 5. Pharoah, Madras.

Zimmermann, J.G. 1932. Über die extrafloralen Nektarien der Angiospermen. Beih. Bot. Centralbl. 49: 99-196.

\section{IDENTIFICATION LIST}

The numbers behind the collector numbers refer to the following taxa:

$1=$ M.actinoneurus Airy Shaw

$2=$ M. attenuatus Airy Shaw

$3=$ M. blumeanus Müll.Arg.

$4=$ M. brevipetiolatus Gage

5 = M. calocarpus Airy Shaw

$6=$ M. cauliflorus Merr.

$7=$ M. claoxyloides (F. Muell.) Müll.Arg.

8 = M. connatus $\mathrm{M}$. Aparicio

$9=$ M. coudercii (Gagnep.) Airy Shaw

$10=M \cdot$ darbyshirei Airy Shaw

$11=$ M. decipiens Müll.Arg.

$12=$ M. didymochryseus Airy Shaw

$13=$ M.dispar (Blume) Müll.Arg.

$14=$ M. eximius Airy Shaw

$15=$ M. glabriusculus (Kurz) Pax \& K. Hoffm.

$16=$ M. glomerulatus Welzen

$17=$ M. hispidospinosus Welzen \& Chayam.

$18=$ M. hymenophyllus Airy Shaw

$19=$ M. khasianus Hook.f.

$20=$ M. korthalsii Müll.Arg.

$21=$ M. lanceolatus (Gagnep.) Airy Shaw
$22=$ M. lauterbachianus (Pax \& K. Hoffm.) Pax \& K. Hoffm.

$23=$ M. leucocalyx Müll.Arg.

$24=$ M. longinervis M. Aparicio

$25=$ M. macularis Airy Shaw

$26=$ M. minimifructus S.E.C. Sierra

$27=$ M. mirus S.E.C. Sierra

$28=$ M. monanthos Airy Shaw

$29=$ M. montanus (Wall. ex Müll.Arg.) Airy Shaw

$30=$ M. oppositifolius (Geiseler) Müll.Arg.

$31=$ M. pierrei (Gagnep.) Airy Shaw

$32=M$. resinosus (Blanco) Merr.

$33=$ M. rufidulus (Miq.) Müll.Arg.

$34=$ M. sphaerocarpus (Miq.) Müll.Arg.

$35=$ M. subcuneatus (Gage) Airy Shaw

$36=$ M. subulatus Müll.Arg.

$37=$ M. tiliifolius (Blume) Müll.Arg.

$38=$ M. trinervius $(\mathrm{K}$. Schum. \& Lauterb.) Pax \& K. Hoffm. 
A series 554: 8; 811: 8; 4634: 26; 4759: 20 - Abbe 10111: 37 - Abeid 803: 30 - J.G. Adam 5783: 30; 6367: 30 - Adduru 93: 32; 180: 32 - Adelbert 361: 37 - Agama 9885: 23 - Aké Assi 4003: 36; 7190: 30; 7226: 30; 7319: 36; 7831: 30; 8750: 30 - Allen 2881: 37 - Alston 14518: 34; 14532: 34; 14679: 13; 15995: 37 - Altmann 528: 37 - Ambriansyah et al. B883: 8 - Amdjah 138: 8; 216: 23 - Amsini 131: 30 - K. Anderson 24: 32 - Andoh 2077: 30 - Andrianantoanina \& Bezara 910: 30 - Andrianantoanina et al. 919: 30 - Angian 10494: 26 - Angus 616: 30 - S. Anthony 463: 20 - Apeleba 1328: 30 - Apostol 3696: 26 - Argent \& Coppins 946: 26 - Argent \& Saridan 94-53: 20 - Argent et al. 139: 32 - ATBP (African Tropical Biodiversity Programme) 491: 30; 722: 30 - Atjeh 7: 37 - Avan 14825: 30 - Axelius 334a: 8; 334b: 8.

Backer 34: 37; 1464: 37; 2287: 37; 3538: 13; 4415: 37; 9186: 3; 17510: 37; 17831: 32; 17920: 37; 18035: 32; 25439: 13; 26802: 37; 27569: 37; 28281: 32; 28489: 32; 31064: 37 - Badré 31: 30 - Bagshawe 765: 30 - Bakhuizen van den Brink Jr. 1810: 13; 2656: 37; 5833: 37 - Balakrishnan 156: 32; 157: 32 - Balansa 3252: 21; 3253: 21 - Baldwin 10859: 36 - Bally 8879: 30; 13753: 30 - Bamps 380: 30 - Barter 1742: 30 - Bartlett \& La Rue 130: 23 - G.L. Bates 648: 30 — Batianoff 11019: 7; 11215: 7; 960955: 7 - Batianoff \& Krieger 6129: 7; 6151: 7; 6255: 7 — Batianoff et al. 11610: 7; 11701: 7; 9812160: 7 - bb series 1992: 3; 2720: 3; 32855: 12 - Beaman 9731: 37 - Beaman et al. 10064: 26 - Bean 6717: 7; 8522: 7; 11036: 7; 18051: 7 - Beesley \& Ollerenshaw 802: 7; 805: 7 - Begmante 2155: 30 - Béguin 935: 37 - Béguin \& Gautier 100: 30 - Béjaud 377: 9 - Berhaut 1256: 30 - Bernardi 8238: 30; 15680: 32 - Beumée 201: 32 - Beveridge 4199: 30 - Bhargava 2355: 32; 2471: 32; 4204: 32 - Bidgood et al. 1709: 30; 1807: 30; 1855: 30 - Biovin 2186: 30 - S.T. Blake 3113: 7 - Bloembergen 4337: 37 - Blume 258: 37; 606: 3; 1366: 3; 1676: 37; 2155a: 3 - Boerlage 146: 37; 320: 37 - Bogor Botanical Garden 5092: 37; IX.A.52: 26; IX.A.52a: 26; IX.C.24: 37; IX.C.24a: 37; IX.C.26: 37; IX.C.75: 32; IX.C.98a: 32; IX.C.115: 37; XII.B.II.1: 26 - Boissier 142: 36 - Bon 2369: 11; 2547: 11; 2574: 11 - Boschproefstation E.1183: 3 - Boyce 993: 19 - Bradtke 139: 37 - Brass 780: 7; 903: 32; 1144: 12; 2594: 37; 14060: 38; 14115: 38; 25451: 37; 28171: 37; 28654: 22; 28701: 22 — Brenan et al. 14506b: 30 - Breteler 808a: 30; 5911: 36 - Breteler et al. 8943: 30 - Brooke 8187: 37; 9896: 26 - R. Brown 3553: 7; 3554: 7 - BRUN series 711: 37; 3231: 26; 16590: 8 - Bryan Jr. 613: 37 - BS series 434: 37; 1056: 32; 1147: 37; 1206: 37; 1252: 37; 1417: 37; 1726: $20 ; 2317: 37 ; 3324: 37 ; 4319: 23 ; 4463: 37 ; 4614: 32 ; 7368: 37 ; 7441: 37 ; 8161: 23 ; 11799$ : 20; 13245: 20; 13909: 6; 14733: 37; 14977: 23; 15351: 37; 15551: 37; 16095: 32; 16308: 37; 16318: $37 ; 16365: 37 ; 17072: 20 ; 17341: 32 ; 17348: 32 ; 17627: 20 ; 18526: 37 ; 20871: 23 ; 21610$ : 32; 22916: 23; 23142: 20; 25547: 20; 26912: 32; 26926: 26; 26941: 37; 27491: 32; 29925: 26; 29927: 26; 30698: 20; 31104: 20; 32267: 32; 32456: 37; 33131: 32; 33460: 6; 36788: 20; 36872: 20; 36928: 20; 43259: 26; 44325: 8; 45904: 32; 45938: 32; 48261: 20; 49099: 23; 75179: 23; 77839: 23; 78339: 32 - BSIP series 1553: 37; 1938: 37; 2646: 37; 5336: 37; 6664: 37; 7248: 37; 8720: 37 ; 8878: 37 ; 10358: 37; 11471: 37; 11664: 37; 12337: 37; 13315: 37; 13390: 37; 13802: 37; 13919: 37; 13969: 37; 14239: 37; 14600: 37; 15233: 37; 16099: 37; 17066: 37; 17103: 37; 17146: 37; 17672: 37; 18249: 37; 18771: 37; 18954: 37 - Buchanan 82: 30 - Burkill 6269: 4 - Burley et al. 706: 20 - Burn-Murdoch 201: 37 - Büsgen 6: 36 - Buwalda 7175: 37; 7178: 37; 7279: 32 - BW series 1075: 12; 8251: 25; 8252: 25; 9124: 38; 9349: 25.

CAHUP series 2992: 37; 22634: 37; 63226: 37; 63885: 37 - Cajano \& Hernaez 1629: 32 - Cameron 20215: 7; 20311: 7 - Canicosa 225: 32; 1904: 32 - Carr 11087: 32; 11699: 12; 13956: 38; 15513: 38 - Carrick 257: 37 - Carvalho 2509: 36 - Carvalho et al. 10237: 36 - P. Castro \& Melegrito 1357: 37; 1516: 26 - CF series 680: 37; 3104: 20 - Chalat 1: 18 - Chamchumroon 2004: 11; 2005: 11; 2006: 11; 2021: 23; 2022: 23; 2023: 23; 2038: 23; 2040: 23 - H. Champion 548: 31 - I.G. Champion \& Sutton 830: 7 - Chand 6009: 19 - C.C. Chang 10590: 21 - Chantaranothai et al. 90/217: 19 - E.G. Chapman \& J.D. Chapman 7126: 30 - Charoenphol et al. 3451: 18 - Chatelain 307: 30 - Chayamarit et al. 2572: 23; 2671: 23 - T.L. Chen 30138: 21 - Chermsirivathana 1490: 23 - Chevalier 16635: 30; 29240: 11; 30565: 9 - W.L. Chew 198: 11; 199: 23; 209: 4; 448: 26; 470: 26; 534: 20; 683: 26; 685: 26 - S.C. Chin 753: 4; 850: 4; 917: 13; 929: 13; 1488: 4; 1488a: 23 - Chongko \& Chomchin 138: 13 - N.K. Chun \& C.L. Tso 44639: 21 - W.Y. Chun 68781: 21 - Church et al. 1200: 20 - Claessens 18: 30 - J. Clemens \& M.S. Clemens 20052: 26; 20075: 26; 20087: 20; 20336: 26; 21239: 20 - M.S. 
Clemens 16104: 32; 18067: 32; 18142: 32; 43299: 7 - Clupip 50: 36 - Coastal Forest Research Programme 20: 30; 21: 30; 475: 30; 609: 30; 694: 30; 1607: 30; 1609: 30; 3500: 30 - Collins 1035: 11; 2069: 23 - Congdon 319: 35; 813: 23; 888: 35 - Corbisier 707: 30; 784: 36; 1130: 30 - Corbisier-Baland 1380: 30 - Craven \& Schodde 999: 32 - Cremers 1038: 30 - H. Cuming 1170: 32; 1171: 32 - Cunningham 529: 37 - Cuong 240: 21 - C. Curtis 183: 24; 1261: 29; 2113: 23; 2257: 23; 2584: 35; 3201: 13 - C.S.S. Curtis 183: 29.

Dalenbah 263: 37 - Dalziel 127: 30; 725: 30 - Darbyshire \& Hoogland 7956: 10 - Darki 1072: 36 - Davidse \& Sumithraarachchi 8076: 32 - Davidson 1388: 35 - Dawos 29843: 14 - De Vogel 1528: 34; 1751: 26; 1817: 26; 2783: 13 - De Voogd 1611: 8; 2320: 37; 2868: 32 - De Wilde 59: 30 - De Wilde \& De Wilde-Duyfjes 6982: 30; 12567: 34; 12722: 34; 13860: 34; 14383: 34; 14818: 34; 14844: 34; 15527: 34; 16512: 34; 18077: 34; 18895: 13; 19203: 34; 20871: 20 — De Wilde \& Jongkind 9334: 30 - O. Degener 14948: 37 - O. Degener \& I. Degener 32148: 37 - O. Degener et al. 13632: 37; 13813: 37; 15506: 37 - Deighton 759: 30; 760: 30; 761: 30; 3514: 36; 4107: 36 - Deistel 167: 36 - L. Deng 2659: 21 — Dibata 138: 30; 141: 30 - Didrichsen 3148: 37; 3184: 37 - Diepenhorst HB 2119: 34; HB 2510: 33 - Dietrich 277: 7; 524 : 7; 576: 7; 1293: 7 - Dinklage 2478: 30; 2479: 30; 2640: 30 - Dissing et al. 1816: 37; 1986: 37 - Djadoek 1517: 3 - Docters van Leeuwen 3902: 13 - Donmes 175: 37 - Drummond \& Hemsley 1305: 30; 3140: 30; 3806: 30 - Du Bois 190: 30 - Dugerdil 122: 30 - Dummer 1085: 30; 4466: 30 .

Eberhardt 3246: 11; 3258: 11; 3272: 11 - Eggeling 1715: 30 - Elbert 2589: 32; 2828: 37; 3698: 37; 4689: 32 - Elmer 6346: 32; 6360: 32; 8296: 32; 12001: 37; 12348: 20; 12545a: 37; 15137: 23; 16540: 32; 16548: 23; 17273: 32; 17421: 32; 17778: 32; 20588: 20; 20747: 8; 20780: 20; 20804: 20; 20905: 20; 20980: 8 - Enchai 10287: 8 - Endert 1183: 3; 5098: 8 - Enti 17: 30; 62: 30 - Esquirol 898: 19; 4354: 21 - Esser 98-73: 11; 98-87: 11; 99-9: 19 - Everist \& Webb 1417: 7 - Evrard 632: 21; 2387: 30.

Faden \& Faden 77-642: 30 - Faden et al. 77-441: 30 - Fakih 152: 30; 571: 30 - Farmar 436: 30 — Faulkener 154: 30; 551: 30; 950: 30; 2348: 30; 2568: 30 - Fay 1921: 30; 3088: 30; 6345: 30; 6612: 30 - FB series 472: 32; 1110: 37; 1462: 37; 1848: 32; 2057: 37; 2317: 37; 2357: 37; 2966: 32; 3011: 32; 3144: 32; 3462: 23; 4114: 37; 6570: 23; 9017: 20; 12212: 37; 17072: 20; 17149: 37; 18876: 32; 18956: 23; 19039: 32; 19296: 32; 21552: 32; 23222: 26; 23363: 23; 23743: 20; 23814: 23; 28123: 32; 28128: 32; 28674: 26; 28729: 23 - Fell 2089: 32 - Fell \& Jensen 2423: 32 - Fell \& McDonald 4353: 32 - Fell \& Stanton 2792: 32; 2802: 32 - Fell et al. 4499: 32 - Fensham 680: 7; 684: 7; 699: 7; 859: 7 - Festo 146: 30 - Festo \& Rugambua 31: 30 - Feuilletau de Bruyn 190: 38 - Flamigni 29: 30; 489: 30 - Floto 7815: 11 - Floyd 6454: 37 - FMS series 2706: 37; 13066: 37; 40005: 35; 44653: 37 - H.O. Forbes 402: 3; 1798: 37 — Forsten 449: 23; 462: 23 - P.I. Forster 520: 7; 2744: 7; 6401: 32; 7538: 7; 7718: 7; 9527: 32; 14426: 32; 14630: 7; 14631: 7; 21737: 7; $29197: 7$ - P.I. Forster \& Booth 25333: 7 - P.I. Forster \& Halford 29663: 7 - P.I. Forster \& Leiper 20060: 7; 28091: 7 - P.I. Forster \& Liddle 8585: 37 - P.I. Forster \& Schmitt 22399: 7; 25185: 7 - P.I. Forster \& Sharpe 9309: 7; 12436 : 7; 12437: 7; 12443: 77 - P.I. Forster \& Symrell 12606: 7; 28926: 7 - P.I. Forster \& Tucker 9262: 7; 10476: 32; 15327: 7; 15386: 7 - Fosberg 24535: 37; 32125: 37; 32380: 37; 40486: 30; 50622: 37 - Fraser 127: 26 - FRI series: see KEP FRI series - Friis 126: 30 - Friis \& Vollensen 1060: 30 - Friis et al. 2555: 30; 3839: 30; 3987: 30; 4105: 30; 7336: 30 - FS series 20393: 20 - G. Fu 5526: 21 - Fuchs 21272: 20 - Fukuoka T-62164: 19.

Gamble 15090: 32 - H.M. Gardner 1463: 30 - R.O. Gardner 6258: 37; 6267: 37; 6511:37 - Garrett 621: 19; 658: 19 - Gaudichaud 136: 23 - Geerling \& Bokdam 171: 30; 171A: 30; 653: 30; 2155 : 30 - Geesink 6679: 11; 6756: 11; 6954: 11; 8060: 19 - Geilinger 1156: 30 - Gerard 1694: 30; 2239: 30 - Gereau 1529: 30 - Gereau \& Hart 4828: 30 - Gereau \& Tanzania Botanical Training Programme 6269: 30 - N. Gibson 604: 7; 968: 7; 987: 7; TOI 301: 7; TOI 486: 7; TOI 487: 7 - G. Gilbert 1514: 30; 1651: 30 - Gillespie 4073: 30; 4140: 30 - Gillett 18646: 30 - Giorgi 1561: 30 - Gobbo et al. 561: 30 - Godefroy 119: 9; 126: 9 - M. Godefroy-Lebeuf 927: 32 - Goklin 2239: 37; 4097: 37 - Gossweiler 4653: 30; 4700: 30; 4709: 30; 5234: 30; 5653: 30; 13859: 30 - Gravendeel et al 522: 8 - B. Gray 5003: 32 - Greenway 4538: 30 - Greenwood 537: 37 - Grimshaw 324: 7 - Gutzwiller 445: 30 - Guymer 361: 7. 
Haegens \& Klazenga 511: 26 - Haenke 153: 37; 161: 37 - Hafashima 289: 30 - Halford Q2016: 7 - Halford \& Crombie Q1329: 7 - Hallier 188: 33; 311: 8; 2816: 8; 4595: 37 - B. Hansen \& Smitinand 12664: 19 - B. Hansen et al. 10896: 19 - C. Hansen 719: 20 - O. Hansen 249: 30 - Harden \& J.B. Williams 81269: 7 - Harder et al. 3383: 30 - Hardial 581: 21 - Harmand 41: 15; 54: 15; 109: 21; 126: 15; 145: 21; 182: 15; 203: 15; 593: 32; 2957: 32 - Harris \& Fay 243: 30; 853: 30 - Hartley 10653: 32; 12276: 22 - Haviland 1019: 37; 1722: 26 - Haviland \& Hose 3258: 26 - Helfer KD 4732: 19; KD 4764: 19 - Helms 124: 7 - Hemingway 162: 20 -Henry 766: 37; 766b: 37; 13023: 19 - Herbst 9392: 37 - Hernay 3361: 32 - Herre 280: 38; 383: 37; 1232: 37 - Heyligers 1758: 32 - Hidayat AH 97: 37 - J.M. Hildebrandt 1144: 30 — Hollrung 376: 37; 409: 37; 426: 32; 781: 38 - Hombron 13: 37 - Hommel 1b: 37; 1d: 13 - Hoogerwerf 44: 37; 127: 37 - Hoogland 4278: 37; 4369: 37 - Horne 597: 37 - Hosokawa 6124: 37; 7284: 37; 9145: 37 - Houtsoorten van den Gedeh 581: 3 - F.C. How 70612: 21 - Hower 905: 30 - Hoy 115: 7 - Hoyle 747: 30 - Humbert 19219: 30 - A.M. Huq \& A.I. Huq 10469: 11 - A.M. Huq \& Mia 10633: 11 - Hyland 4795: 7; 6348: 32; 9102: 7; 12443: 32; 12444: 32; 12898: 32; 15171: 7 .

Iboet 1921-419: 3 - Irving 153: 30 .

Ja series 7047: 3; 7371: 3 - Jackson 1497: 30 - K.C. Jacob 17556: 32 - Jacobs 4814: 13; 7769: 37; 8372: 3 - Jaheri 138: 8 - J.W.A. Jansen 2517: 30 - Jayasuriya 1098: 32; 1296: 32; 1751: 32 - Jessup 45: 7 - Jessup \& W.J.F. McDonald 109: 7 - Jessup et al. 2867: 32 - R.J. Johns 7739: 38 - W.T. Jones 3831: 7; SN5557: 7.

Kadim \& Mahmood 88: 35 - Kadim \& Noor 656: 13 - Kairo 147: 22 - S.F. Kajewski 2347: 37 - Z. Kajewski 56: 7 - Kalshoven 1609: 32 - Kamis 2950: 37; 2951: 37 - Kanehira 438: 37; 1855: 37; 2294: 37 - Karim \& Shah 96: 4 - Karta 287: 37; 334: 37 - Kartawinata 91: 33; 885: 26 - Katende 1413: 30 - Kato \& Wiriadinata B-5524: 20 - Kato et al. B-11760: 26; B-11888: 8 - Kaudern 202: 37; 491: 32; 492: 32 - Kayambo 1636: 30 - Keasy 22263: 30 — Keay 22269: 30 - Keith 52: 31; 9480: 37 - Kennedy 1831: 36; 1921: 36; 1940: 36; 1944: 30; 2090: 36 - KEP series 3104: 20; 14619: 13; 24212: 35; 32653: 13; 38639: 20; 46727: 23; 49061: 8; 94598: 13; 110159: 4 - KEP FRI series 1191: 29; 1195: 29; 1601: 4; 2499: 1; 3544: 28; 4733: 29; 5533: 23; 5839: 4; 6596: 35; 13243: 20; 13517: 14; 13685: 29; 15372: 23; 16310 : 20; 16311: 20; 17911: 1; 20227: 14; 20722: 29; 23343: 29; 31475: 13 - Kerr 1161: 19; 2086: 11; 2326: 31; 2353: 15; 2722: 19; 2950: 31; 3139: 19; 3244: 31; 3552: 19; 5333: 19; 5686: 21 ; 5686a: 21; 5994: 9; 5994a: 9; 6326: 19; 7009: 9; 7009a: 9; 7212: 13; 8617: 21; 8623: 15; 8626: 31; 9052: 21;9122: 21; 9610: 11; 10349: 19; 10594: 31; 10597: 31; 10750: 11; 10789: 23; 10789a: 23; 10798: 31; 11497: 23; 11497a: 23; 11802: 32; 12284: 4; 12378: 18; 12389: 18; 12406: 18; 12574: 35; 12635: 37; 12840: 9; 13029: 37; 13109: 37; 14607: 23; 15331: 11; 17279: 4; 17419: 35; 17965: 5; 18260: 23; 18942: 35; 18974: 35; 19058: 23; 19104: 4; 19290: 11; 19290a: 11; 19504: 9; 19835: 27; 20012: 21; 20326: 11; 20355: 11; 20466: 21; 20502: 31; 21572: 31; 21584: 23; 21687: 23 - Kersting A290: 30 - Keßler \& Arbainsyah B1361: 20 - Keßler \& Koonthunthod PK3276: 19 - Keßler et al. PK1604: 26 - Kiew 2392: 20; 3575: 11; 3579: 23 - Kiew \& Anthonysamy 2951: 4 - King's collector 263: 32; 921 : 13; 1464: 29; 1675: 29; 1699: 23; 6156: 13 - Kjellberg 946: 23; 1239: 37; 3147: 37 - KL series 1824: 35; 2129: 35; 3104: 37; 3229: 37; 3960: 20 - Klaine 371=439: 36; 2286 (FI herb, not C): 36; 2717: 36; 2730: 36; 2783: 36; 2861: 36 - Kleinhoonte 785a: 32 - Knecht 756: 30 - Koelz 30579: 19 - Köhler \& Vent 23: 30 - Køie 1816: 37 - Køie \& Olsen 1986: 37 - Kool 161: 13 - Koonthunthod et al. 517: 16; 524: 16 - Koop 130: 34; 200: 34 - Koorders 2081: 32; 2390: 37; 2391: 37; 2519: 37; 2544: 3; 2545: 3 ; 2546: 3 ; 2547: 3 ; 2549: $3 ; 2553: 3 ; 2568: 37 ; 2578: 37 ; 2579: 37 ; 2735: 33 ; 10032$ : 32; 10034: 32; 10322: 13; 11165: 3; 12279: 3; 16932: 37; 16947: 26; 16955: 37; 16970: 26; 20503: 37; 25187: 32; 25189: 32; 27385: 33; 27396: 13; 28936: 33; 29985: 37; 30532: 3; 30533: 13; 30538: 13 ; 30541: 13 ; 30543: 13; 30639: 3; 30640: 3 ; 33014: 3 ; 33119: 3; 38795: 13; 39030: 3 ; 39844: 37; 40132: 3; 40209: 32 - Kornassi 636: 37; 765: 37 - Koroiveibau 8799: 37; 13664: 37 - Korthals 986: 3 - Kostermans 7A: 37; 741: 37; 1963: 33; 13762: 26; 13985: 8; 19302: 3; 19317: 37; 19326: 3; 21345: 26; 21548: 8; 21595: 20 - Kostermans \& Van Woerden 35: 37; 93: 37 - Kostermans et al. 50: 32; 51: 32; 263: 33; 317: 33; 344: 32 - Koyama et al. 15.418: 19; 15.552: 19 - Kramer 6211: 32 - Kuruvoli 14320: 37; 14643: 37; 16068: 37 - S. Kurz 1242:15; 26073: 32 - W.S. Kurz 5367: 37. 
Lace 2837: 32; 4218: 15 - LaCroix 4960: 30 - LAE series 61115: 12; 87156: 12 - Lam 1030: 38; 2283: 13; 3351: 37 — Lam \& Meeuse 6117: 30; 6118: 30 - Laman et al. 338: 8; 676: 8 K. Larsen 1363: 11; 1531: 11; 1607: 31; 1608: 31; 1611: 11; 2207: 31; 2208: 31; 2463: 31; 3243: 11; 3264: 21 ; 3367: 11; 3609: 9; 8331: 31; 9740: 5; 31054: 23; 31180: 11; 31247: 18; 31276: 23; 31279: 11; 31559: 21; 32115: 9; 32469: 5; 41114: 29; 41666: 29; 41716: 23; 43078: 23; 43351: 23; 44566: 19; 44703: 19; 45390: 11; 45445: 23 - K. Larsen \& S.S. Larsen 32796: 29; 33703: 11; 34431: 19 - S.K. Lau 3232: 21; 3325: 21; 3438: 21; 6194: 21 - Laumonier 4001: 13 Lavaco 1301: 30 - Le Cussan 460: 32 - Le Testu 604: 30; 1460: 30; 1461: 30; 4722: 30; 8198: 30 - Lebler \& Durrington 11: 7 - Lebrun 1264: 36; 1484: 30; 1544: 36; 2723: 30 - Lecomte \& Finet 254: 11; 274: 11; 1707: 21 - Ledermann 10417: 38; 14514: 37 - Leeuwenberg 2157: 30; 3098: 30; 4088: 30; 4089: 30; 4494: 36; 4495: 36; 5888: 30; 9662: 30 - Leonard 350: 30; 372: 30; 373: 30; 428: 36; 1381: 30 - Letouzey 1821: 30 - M.X. Li 692: 19 - Z.T. Li 601013: 19 - Z.T. Li \& Xing 6057: 21 - C.F. Liang \& P.L. Wu 32553: 19 - Liben 3446: 30 - Linder 1713: 36; 1718: 36; 1722: 36 - Lisowski 40568: 30 - Loc et al. 1642: 21 - Loher 4644: 32; 4796: 32; 6854: 32; 13930: 32 - Lojtnant \& Niyomdham 160: 19; 226: 19 - Lörzing 4032: 34; 4258: 34; 4270: 34; 5232: 33; 7398: 13; 14471: 23; 14987: 33; 15212: 13; 15234: 34 - J. Louis 259: 30; 927: 30; 940: 30; 943: 30; 3609: 30; 7086: 30; 10268: 30; 10462: 30; 11514: 30; 12457 : 30 - Lowe 3573: 30; 3668: 30; 3785: 36 - Luchun Exped. 1404: 21.

Madsen et al. 3290: 30 - Magbagbeola et al. 86: 30 - Mahyar 1197: 8 - Maitland 368: 36 - Malaisse 11142: 30; 13630: 30 - Mann 260: 36; 261: 36 - Manning 156: 30; 230: 30 - Maradjo 101a: 3; 431: 3 - Maradjo \& Ichlas 127: 3 - Marcan 793: 21; 2208: 31; 2335: 31; 2630: 31; 2656: 31 - Maskuri 910: 37; 934: 37 - Massawe 37: 30 - Maxwell 71-256: 5; 72-290: 31; 72-357: 11; 72-522: 5; 73-46: 5; 73-47: 5; 74-825: 11; 74-826: 11; 75-42: 11; 76-321: 21; 80-27: 37; 85-457: 29; 86-352: 23; 86-420: 4; 86-506: 23; 87-173: 18; 87-176: 31; 88-84: 19; 88-148: 19; 88-511: 31 ; 88-518: 31; 88-614: 19; 89-74: 19; 89-626: 31; 91-417: 19; 92-316: 31; 93-1213: 19; 94-102: 19; 94-507: 11; 95-467: 11; 96-639: 21; 96-920: 21; 97-102: 19; 97-141: 19; 97-206: 19; 97-1200: 21; 98-321: 17; 98-510: 21; 98-520: 21; 98-1365: 17; 03-101: 19 - J.A. McDonald \& Afriastini 3307: 3; 3358: 37 - J.A. McDonald \& Sunaryo 4394: 37 - W.J. McDonald \& J.B. Williams 3725: 7 - A. Mechows 556: 30 - Meebold 7845: 15; 9892: 7 - Meijer 1765: 8; 2452: 8; 10116: 37; 15165: 30 - Melville 3652: 7 - E.D. Merrill 1017: 37; 1183: 37; 2555: 32; 2643: 32; 2720: 37; 3032: 32 - Merrill Species Blancoanae 50: 37; 485: 32 - Messedagh 1: 30 - Michael 2099: 7; 2154: 7 - Michels 2: 30 - Middleton et al. 469: 23; 885: 11; 1065: 11; 1104: $11 ; 1226: 11 ; 1278: 11 ; 1320: 31 ; 1368: 11 ; 1484: 23 ; 2122: 23 ; 2135: 11 ; 2794: 13 ; 2795: 13$ — Milne-Redhead \& Taylor 7574: 30; 7575: 30; 7576: 30 - Mitsuta T-47587: 19 - Molesworth Allen 4269: 13; 4644: 4 - Moriarty 909: 7 - Morrison 253: 37; 298: 37 - Morton A286: 36; 6127: 30; 6566: 36 - Motuba 15243: 36 - Mousset 1138: 13; 1141: 33 - Murata et al. B-2702: 37; J-535: 20; T-16544: 9; T-16884: 11; T-37185: 21; T-42498: 19 - Muslihat \& Momo 147: 3 - Musser 715: 20 - Mwangulango \& Kishe 216: 30; 226: 30.

Nagamasu 3192: 33 - Nair 3675: 32 - Nakkan 114: 31 - Nangkat 331: 8 - Napper 1274: 30 - Nemba \& Thomas 357: 36; 513: 30 - Newman et al. 1059: 23; 1196: 11 - NGF series 3862: 38; 5090: 7; 5095: 7; 8255: 12; 13094: 12; 13116: 12; 13167: 37; 17486: 12; 17573: 7; 21753 : 37; 24786: 38; 25408: 32; 26634: 37; 27781: 2; 29430: 37; 34293: 38; 37524: 38; 43080: 37 — Nicolson 1639: 5 - Nielsen 877: 25 - Niyomdham 7: 19; 15: 19; 34: 19; 263: 32; 3018: 11 - Noe 98: 21 - Noor \& Samsuri 17: 33 - Nooteboom 3389: 32 - Nooteboom et al. 798: 19 - Nusbaumer \& Kouamé 517: 30 - Nusbaumer et al. 546: 30.

Oates 95: 32 - Odewo \& Adedeji 362: 30 - Odewo \& Binuyo 271: 30 - Ogata 10243: 13; 10919: 32 - Oldeman 787: 30; 977: 30 - Olsen 514: 37 - Orolfo 1449: 32 - Osmaston 2627: 30.

Palee 283: 31 - Pancho 1593: 23 - Pancho \& Barderaz 5619: 32 - B.E.V. Parham 7153: 37; 7219: 37 - H.B.R. Parham 27a: 37; 27b: 37 - J.W. Parham \& Berry 17087: 37 - Parnell et al. 95-98: 19; 95-416: 11; 95-416a: 11; 95-534: 23 - Pendleton 432: 37 - Pereira et al. 595: 37 - Pervillé 360: 30; 486: 30 - Pételot 144: 11; 1277: 11; 1417: 11; 1418: 11 - Peters 1032: 20 - Phattarahirankanok et al. 17: 18 - Phengklai et al.7053: 19; 7086: 19; 7113: 19; 10941: 19 - Phengnaren 468: 21; 658: 21 - Pierre 2957: 21; 5314: 13; 5315: 32; 6254: 31 - Pilz 1833: 30; 1881: 30; 1998: 36; 2007: 30; 2126a: 30; 2126b: 30 - Pirozynki 365: 30 - Playfair 324: 37 - Pleyte 848: 37 - PNH series 2438: 37; 6390: 37; 6742: 32; 8470: 32; 9009: 32; 9686: 37; 
9749: 32; 10414: 26; 11056: 23; 13573: 26; 13778: 37; 13809: 32; 13829: 37; 15701: $37 ; 15703$ : 32; 15775: 20; 17050: 23; 17101: 32; 17987: 32; 18036: 32; 18136: 32; 19163: 37; 19853: 32; 32818: $37 ; 34029: 32 ; 36272: 37 ; 36599: 37 ; 36808: 37 ; 38086: 26 ; 39047: 37 ; 40166: 20 ; 40494$ : 32; 40739: 32; 42093: 26; 42102: 26; 42354: 26; 80803: 23; 80807: 23; 91510: 32; 97743: 37; 97869: 37; 150062: 37 - Poilane 3031: 21; 5631: 21; 5686: 9; 6036: 21; 8853: 11; 8935: 21; 9367: 32; 12523: 11; 16385: 21; 16473: 21; 19693: 32; 19704: 32; 20585: 11;21267:9 - Poilecot 2194: 30; 4223: 30; 4238: 30 - Polhill \& Paulo 739: 30; 799: 30 - Polhill \& Robertson 4787: 30 - Pollock 1134: 7 - Pooma 1669: 15 - Pooma et al. 2636: 16; 2662: 16; 3817: 21; 3856a: 9; 3856b: 9; 4065: 31; 4066: 31; 4300: 13; 4332: 13 - Posthumus 930: 3 - PPI series 2966: 37; 3940: 32; 3977: 32; 4051: 26; 4453: 37; 5479: 37; 6596: 37; 7297: 26; 7353: 26; 9785: 32; 9798: 32; 9882: 32; 9937: 32; 10070: 23; 10276: 32; 14737: 23 ; 14754: 23 ; 15540: 37; 15565: 37; 17261: 32; 17340: 37; 23305: 20; 25230: 32; 29168: 37; 29238: 37; 29521: 32; 37573: 32 - Preuss 480: 30 - Put 698: 23; 699: 23; 884: 32; 1044: 23; 1309: 35; 1519: 32; 1623: 23; 1633: 11; 1778: 31; 1864: 21; 2038: 21; 2169: 9; 2483: 11; 2671: 9; 3215: 31; 3765: 19; 3946: 9; 4179: 11; 4311: 21.

Raap 318: 33 - Rabil 169: 11 - Raes et al. 578: 26; 589: 26; 633: 26 - Rahayu 349: 3 - Rahmat si Boeea (= Rahmat si Toroes) 840: 33; 4338: 20; 4510: 20 - Ramos 434: 37 - Rappard 169: 33 - A. Raynal \& J. Raynal 9874: 30 - Reitsma et al. 3304: 30 - RHT series 23885: 32; 27385: 32 - Ridley 2675: 13; 7647: 35; 8201: 13; 8322: 23; 8528: 13; 11910: 13; 14694: 13; 14904: 23; 15190: 11; 15222: 4 - Ridsdale 1878: 26 - Rijksen 28773: 34 - Rinehart 266684: 37 - Risopoulos 505: 30 - Riswan \& Afriastini J049: 37 - Ritchie 1256: 32 - Robertson 3648: 30; 3874: 30 - G. Roberty 7034: 30; 7040: 30; 12538: 30; 12769: 30; 12816: 30; 12847 : 30; 13540: 30; 13925: 30; 14119: 30; 18018: 30; 18027: 30 - M.G. Roberty 1602: 30; 1611: 30; 1657: 30 - Robinson 367: 37; 368: 37 - Robinson \& Kloss 6173: 35 - Robyns 749: 36 - Rogerson 1049: 37 - Romero Monteiro \& Murta 352: 30 - Rood \& Hardie 4348: 7 - RSS series 2810: 37 - Rudatis 107a: 36 - Rutten 33: 8; 59: 8; 105: 37; 107: 37; 112a: 37; 117: 8; 517: $8 ; 631: 8 ; 1787: 37$.

S series 3389: 24; 4643: 26; 8348: 37; 11100: 20; 13778: 26; 14722: 20; 14867: 26; 17914: 37; 20940: 26; 21702: 26; 21730: 26; 23440: 20; 24384: 20; 25113: 37; 25977: 24; 25990: 24; 26206: 24; 28132: $20 ; 28245: 20 ; 30355: 26 ; 31693: 26 ; 31823: 26 ; 31910: 26 ; 34195: 26 ; 36689: 20 ; 38232$ : 26; 40198: 26; 41061: 26; 41952: 37; 43735: 20; 46235: 26; 48717: 37; 49591: 26; 49657: 26; 53714: 20; 62305: 26; 62330: 26; 64620: 20; 82078: 26; 82080: 20; 82162: 26; 82163: 26 - SA series 542: 4; 595: 4; 620: 13; 950: 13 - Saakov 173: 33 - Saanan 118: 37 - Sales 4262: 20 - SAN series 3764: 37; 15035: 32; 22234: 20; 23536: 20; 24219: 20; 24227: 20; 25062: 20; 26022: 26; 26533: 20; 27556: 37; 28653: 20; 29303: 20; 29514: 20; 30086: 20; 30112: 8; 30419: 20; 31489: 20; 31555: 20; 31631: 20; 31678: 20; 31731: 20; 33219: 8; 33958: 20; 33969: 20; 33985: 20; 36002: 20; 36326: 20; 37075: 20; 37409: 20; 38003: 8; 40260: 20; 42055: 20; 42227: 26; 43000: 8; 43090: 26; 47784: 20; 47806: 20; 53105: 20; 54647: 20; 54909: 20; 56235: 20; 57293: 20; 60147: 20; 61334: 20; 61504: 37; 61510: 37; 66556: 26; 66584: 26; 66762: 8; 67250: 20; 67397: 20; 67446: 8; 67531: 20; 68382: 20; 75332: 20; 75344: 20; 75728: 20; 78477: 20; 79203: 20; 80917: 20; 81409: 20; 81638: 8; 82177: 20; 83929: 20; 86417: 37; 89264: 20; 90972: 26; 91129: 20; 94931: 20; 96445: 8; 96916: 20; 97005: 20; 97300: 26; 102085: 37; 102827: 37; 103161: 37; 104066: 8; 107175: 20; 107326: 20; 108134: 20; 108575: 8; 108576: 20; 108626: 20; 109202: 20; 110124: 20; 111805: 20; 115272: 37; 115692: 20; 116945: 20; 124235: 20; 124842: 20; 126017: 37; 126253: 37; 126802: 37; 126964: 37; 135968: 20; 142561: 20 - SandermanOlsen 754: 8 - Sands 6166: 25 - B. Sangkhachand 9: 31; 704: 23; 705: 23 - P. Sangkhachand 1490: 23; 1579: 13; 1757: 13; 1785: 29 - Santisuk 193: 11; 6684: 32 - Santisuk \& Nimanong 365: 21 - Santos 4531: 32 - Sapin 2912: 30 - Sarip 111: 33 - Saunders 951: 38 - Sauveur 3361: 38 - Schlechter 12402: 36; 12766: 30; 13025: 30; 17916: 22; 17919: 22 - Schlieben 1669: 30; 2679: 30; 5318: 30; 5681: 30; 5687 (left specimen, B): 30 - Schmidt et al. 1956: 30 - Schmutz 522: 32; 525: 33; 606: 33; 812: 33; 826: 32; 6089: 32 - Schodde 2365: 38; 2509: 12; 5368: 12 - Schodde \& Craven 4407: 12 - Scholz et al. 39: 30 - Schürmann 133: 34; 136: 34; 152: 34 - Scott-Elliot 4461: 30 - Seemann 407: 37 - Semsei 634: 30 - SF series 201: 37; 965: 37; 997: 13; 1559: 11; 4227: 23; 14488: 33; 15203: 37; 18377: 14; 18476: 13; 18884: 14; 18956: 20; 19219: 37; 19497: 13; 19497a: 23; 22697: 13; 22698: 13; 22733: 37; 22819: 11; 
23020: 11; 23122: 23; 23786: 13; 25211: 13; 25814: 37; 28467: 37; 29041: 20; 29143: 23; 29851 : 37; 30250: 20; 31934: 20; 32002: 20; 35096: 29; 35238: 11; 35375: 23; 40805: 37 — Shah \& Ali 2854: 13 - Shah \& Noor 2017: 23 - Sharpe 3681: 7 - Shetty 27941: 32 - Shimizu \& Toyokuni T-27361: 23 - Shimizu et al. T-7703: 31; T-7926: 11; T-7928: 11; T-7961: 11; T-8721: 21; T-8726: 21; T-8781: 31; T-11647: 19; T-19428: 11; T-27084: 21; T-27085: 21; T-27425: 18 — Sidek Bin Kiah \& Maulod Bin Elin 563: 14 - Sidiyasa 440: 26; 525: 26; 1213: 8; $3547: 26$ - Sidiyasa \& Arifin 1975: 26 - Sidiyasa et al. 2815: 25 - Sinclair 9853: 4; $9856: 4$ - Sinclair et al. 9297: 8 - Sirirugsa 653: 37 - Slik et al. 12: 26 - SMHI series 846: 32; 2021: 32 — Smith \& Rant 693: 33 - A.C. Smith 6369: 37; 6604: 37; 6710: 37 - C. Smith 76: 36; 79: 36 - L.S. Smith 4105: 7; 4109: 7; 14366: 32; 14451: 32 - Smitinand 2938: 35; 4834: 21; 11500: 4 - Smitinand et al. 1222: 23; 1222a: 31; 10259: 19 - Soejarto 78: 33 - Soejarto \& Quang 13420: 11; 13422: 11 - Soejarto et al. 5791: 11; 11674: 21 - Soepadmo 297: 13; 736: 28 - Sohmer 8043: 32 - Sørensen et al. 3009: 19; 7815: 11 - Sosef et al. 772: 30; 829: 30 — Soyaux 155: 30 - Speck 1688: 7 - Squires 786: 32; 803: 32 - Staner 1431: 30 - Stäuble 532: 30; 791: 30; 1036: 30 - Staudt 597: 30 - Stone 6947: 23; 6957: 35; 7478: 23; 9178: 13; 9526: 23; 14317: 35 - Stone \& Wycherley 8974: 13 - Sunderland 1266: 30 — Suwanakoses 672: 23; 1318: 21 - Szafranski 1300: 30.

Takamatsu 1127: 37; 1232: 37; 1586: 37 - Talbot 621: 36; 623: 36; 655: 36; 697: 30 - Talmy 135 : 15; 377: 15 - Tandom 4126: 26 - Tanner 3101: 30; 3400: 30; 3471: 30; 3543: 30; 3620: 30; 3970: 30 - Tanthana \& Boonkongchart 35: 11 - Tateishi et al. 1024020: 21 - Taylor 512: 32 — Teijsmann HB 3950: 13; HB 4190: 37; HB 8606: 8; HB 10748: 32; HB 13912: 32 — Telford \& Butler 9036: 7; 9076: 7; 9098: 7 - Teng 90803: 19 - Thaworn 259: 23 - D.W. Thomas 2485: 30; 2486: 30; 2534: 36; 2535: 36; 7410: 30; 7411: 30 - N.W. Thomas 888: 30; 2074: 36; 2640: 30; 10286: 30 - P. Thomas et al. 19: 19; 63: 19 - Thorel 2027: 9; 2142: 15; 2265: 15 - Thorne 20025a: 7 - Thwaites CP 2108: 32 - C.S. Tisserant 137: 36; 511: 36 - R.P. Tisserant 489: 30; 3312: 30; 3762: 30 - Tothill \& Tothill F445: 37 - Tracey 14407: 32; 14428: 32 - Tsai 54549: 21 - Tuyama 1168: 25; 9432: 37.

UNESCO series 226: 33 - Unwin 38: 36 - UPNG series 3617: 7; 5366: 7; 5367: 7; 5369: 7.

Valbuena 23: 37 - Valera 8841: 23 - Van Balgooy 4832: 37 - Van Beusekom \& Phengklai 1246: 31 - Van Beusekom \& Santisuk 2700: 11; 2703: 31 - Van Beusekom et al. 4480: 21 — Van Borssum Waalkes 157: 37; 201: 37; 741: 37; 762: 37 - Van der Meer 806: 30 — Van der Werff et al. 17100: 11 - Van Schaik \& Van Noordwijk 117: 34 - Van Steenis 626: 13; 644: 13; 2346: 3; 2675: 13; 4723: 37; 7611: 33; 7704: 37; 11301: 37; 11393: 37; 18205: 32 - Van Welzen 501: 30 - P. Vanpruk 516: 31 - Vaughan 20:30; 21: 30; 45: 30; 2751: 30 - Vavryn 30: 7 - Verdcourt 5268: 30 - Verheijen 2446: 37; 2649: 32; 2809: 32 - F. Vermoesen 2368: 30 - M.C. Vermoesen 2451: 36 - Verreaux 56: 7; 592: 7 - J.E. Vidal 4522: 15; 5764: 5 - Vidal y Soler 129: 37; 311 : 32; 322: 32; 340: 32; 583: 23; 886: 37; 3699: 32; 3753: 32; 3755: 32; 3760: 23; 3760bis: 23; 3767 : 32; 3871: 32; 3876: 32 - Vogel 11: 30 - Vollesen MRC 3171: 30; MRC 4403: 30.

Waas 589: 32; 763: 32 - Waas \& Tirvengadum 824: 32 - Wagemans 1223: 30 - Walker 779: 32; 1217: 32 - Wallich 7723B: 29; 7725: 11; 7751B: 32; 7752: 32; 7828: 29; 8009: 11 - C.W. Wang 72758: 19; 72888: 19; 73228: 19; 76467: 19 - Warburg 11695: 32; 12320: 32; 12322: 32; 13996: 32; 17276: 37 - Warnecke 319: 30 - Waterhouse 222: 37; 302: 37; 868: 37 - Weatherhead 489: 7 - L.J. Webb \& J.G. Tracey 7823: 32; 11885: 32; 13340: 32; 13806: 32; 13807: 32; 13808: 32; 13809: 32 - Webster \& Hildreth 15014: 7 - Weis 346970: 37 - Welwitsch 342: 30 - Wenzel 1336: 20; 1338: 20; 1456: 20; 3285: 26 - Wheatley 16: 36; 213: 30; 660: 36 - C.T. White 747: 7; 8697: 7; 11413: 7; 12570: 7 — Whitford 26: 32; 386: 32; 1585: 23 — Whitmore 3071: 20 - Whitmore \& Wardani 3367: 34 - Widjaja 97: 33 - Widjaja \& Hamzah 3092: 25 - Wight KD2614: 32; KD2672: 32 - J.W.G. Wild 404: 30 - R.S. Williams 2891: 32 - Winckel 1795: 3 - Wingfield 4312: 30 - Winit 290: 11; 445: 11; 446: 31; 446a: 31; 559: 23; 576a: 11; 599: 31; 689: 15; 1333: 19; 1607: 15 - H. Winkler 52: 30; 435: 36; 558: 30 - Wirawan 402: 33; 613: 32 - Wiriadinata 811: 20; 816: 20 - D.D. Wood 1217: 8.

T.T. Yu 16246: 19; 18162: 19 - Yusuf \& Wahyono 35: 37.

Zainudin 392: 23; 1810: 23; 3654: 13; 4568: 20; 5505: 13 - Zainudin et al. 1520: 37 - Zenker 1399: 30 - T. Zhu 617: 19 - Zollinger 1098*(G mixed): 33; 1098*(G mixed): 13; 1554a: 13; 1616: 33; 1814: 37; 2733: 37; 3651: 13; 3804: 32 - Zwickey 419: 20. 


\section{INDEX TO NAMES}

The numbers behind the names refer to the species number in this article; dub. = dubious species; excl. $=$ excluded species. Accepted names are in roman type; synonyms in italics and new combinations in bold.

Acalypha? dentata Schumach. \& Thonn. 30

Adelia papillaris Blanco 37

resinosa Blanco 32

Axenfeldia intermedia Baill. dub.

Claoxylon cordifolium Benth. 30

muricatum Wall. ex Wight 32

Coelodiscus coudercii Gagnep. 9

eriocarpoides Kurz 11

glabriusculus Kurz 15

glabriusculus auct. 9

lanceolatus Gagnep. 21

lauterbachianus Pax \& K. Hoffm. 22

montanus Wall. ex Müll.Arg. 29

muricatus (Wight) Gagnep. 32

muricatus auct. 11

pierrei Gagnep. 31

subcuneatus Gage 35

trinervius (K. Schum. \& Lauterb.)

Pax 38

Croton acuminatus Lam. 37

enantiophyllus K. Schum. 37

muricatus Wall. 32

oppositifolius Geiseler 30

tiliifolius Lam.

var. aromaticus auct. 37

Echinocroton F. Muell. [p. 34]

claoxyloides F. Muell. 7

Echinus claoxyloides (F. Muell.) Baill. 7 var. cordata Baill. 7 oppositifolius (Geiseler) Baill. 30

Halecus litorea Rumph. 37

Lasiococca brevipes (Merr.) Welzen \& S.E.C. Sierra excl.

Mallotus Lour. sect. Axenfeldia Pax \& K. Hoffm. [p. 34] sect. Echinocroton (F. Muell.)

Pax \& K. Hoffm. [p. 34] sect. Plagianthera (Rchb.f. \& Zoll.)

Pax \& K. Hoffm. [p. 34]

sect. Rottleropsis Müll. Arg. [p. 34]

actinoneurus Airy Shaw 1

andamanicus Hook.f. 32

attenuatus Airy Shaw 2

aureo-punctatus (Dalzell) Müll.Arg. dub.

blumeanus Müll.Arg. 3

bracteatus Hook.f. dub.

brevipes Merr. excl.

brevipetiolatus Gage 4
(Mallotus)

buettneri Pax dub.

calocarpus Airy Shaw 5

cauliflorus Merr. 6

chevalieri Beille 30

claoxyloides (F. Muell.) Müll.Arg. 7

var. cordatus (Baill.) Airy Shaw 7

var. glabratus Domin 7

clellandii Hook.f. 15

connatus M. Aparicio 8

coudercii (Gagnep.) Airy Shaw 9

cuneatus Ridl. 11

darbyshirei Airy Shaw 10

decipiens Müll.Arg. 11

didymochryseus Airy Shaw 12

dispar (Blume) Müll.Arg. 13

var. lasioneurus Müll.Arg. 13

var. psiloneurus Müll.Arg. 32

dispar auct. 35

eriocarpus auct. 29

esquirolii H. Lév. 19

eximius Airy Shaw 14

filiformis Hook.f. 19

glabriusculus (Kurz) Pax \& K. Hoffm. 15

glomerulatus Welzen 16

hainanensis S.M. Hwang 21

hispidospinosus Welzen \& Chayam. 17

hymenophyllus Airy Shaw 18

intermedius (Baill.) N.P. Balakr. dub.

khasianus Hook.f. 19

korthalsii Müll.Arg. 20

lanceolatus (Gagnep.) Airy Shaw 21

lauterbachianus (Pax \& K. Hoffm.)

Pax \& K. Hoffm. 22

lawii Müll.Arg. dub.

leucocalyx Müll.Arg. 23

longinervis M. Aparicio 24

lullulae Airy Shaw 22

macularis Airy Shaw 25

minimifructus S.E.C. Sierra 26

mirus S.E.C. Sierra 27

monanthos Airy Shaw 28

montanus (Wall. ex Müll.Arg.) Airy Shaw

29

moritzianus Müll.Arg. 33

var. scaber Müll.Arg. 33

moritzianus auct. 8

muricatus (Wight) Bedd. 32 
(Mallotus)

muricatus (Wight) Müll. Arg. var. genuinus Pax \& K. Hoffm. 32 var. walkerae (Hook.f.) Pax \& K. Hoffm. 32

muricatus auct. 32

oppositifolius (Geiseler) Müll.Arg. 3, 30

var. genuinus Müll.Arg. 30

var. glabrata Müll.Arg. 30

var. integrifolius Müll.Arg. 30

var. lindicus (Radcl.-Sm.) Radcl.-Sm. 30

var. pubescens $\operatorname{Pax} 30$

forma dentatus (Schumach. \& Thonn.)

Pax \& K. Hoffm. 30

forma glabratus (Müll. Arg.) Müll. Arg. ex Pax \& K. Hoffm. 30

forma lindicus Radcl.-Sm. 30

forma polycytotrichus Radcl.-Sm. 30

forma pubescens (Pax) Pax \& K. Hoffm. 30

palauensis Hosok. 37

papillaris (Blanco) Merr. 37

petanodon Airy Shaw 20

pierrei (Gagnep.) Airy Shaw 31

playfairii Hemsl. 37

polyneurus Hook.f. 19

ponapensis Hosok. 37

repandus auct. 37

resinosus (Blanco) Merr. 32

var. cuneatus (Ridl.) N.P. Balakr. \&

Chakrab. 11

var. subramanyamii (J.L. Ellis) Chakrab. dub.

rufidulus (Miq.) Müll.Arg. 33

rufidulus auct. 8

sanguirensis $\operatorname{Pax} \& \mathrm{~K}$. Hoffm. dub.

smilaciformis Gage dub.

spec. nov. 27

spec. nov. 117

spec. nov. 232
(Mallotus)

sphaerocarpus (Miq.) Müll.Arg. 34

spinihispidus Welzen \& Chayam. 17

spodocarpus Airy Shaw 9

stenanthus Müll.Arg. 32

subcuneatus (Gage) Airy Shaw 35

subramanyamii J.L. Ellis dub.

subulatus Müll.Arg. 36

tiliifolius (Blume) Müll.Arg. 37

trinervius (K. Schum. \& Lauterb.)

Pax \& K. Hoffm. 38

tristis Pax \& K. Hoffm. 15

viridis Welzen \& Chayam. 32

walkerae Hook.f. 32

var. laxiflora Hook.f. 32

zollingeri Müll.Arg. 33

zollingeri auct. 37

Plagianthera Rchb.f. \& Zoll. [p. 34] oppositifolia (Blume) Rchb.f. \& Zoll. 3

Ricinocarpus dentatus (Schumach. \& Thonn.) Kuntze ex Govaerts, Frodin \& Radcl.-Sm. 30

Rottlera acuminata (Lam.) A. Juss. 37

affinis Baill. 7

aureopunctata Dalzell dub.

blumeana (Müll.Arg.) Scheff. 3

blumei Decne. 37

dentata (Schumach. \& Thonn.) Baill. 30

dispar Blume 13

dispar auct. 33

korthalsii (Müll.Arg.) Scheff. 20

moritziana (Müll.Arg.) Scheff. 33

muricata (Wight) Thwaites 32

oppositifolia Blume 3

rufidula Miq. 33

sphaerocarpa Miq. 34

tiliifolia Blume 37

Syndyophyllum trinervium K. Schum. \& Lauterb. 38 